\title{
CUBIC AND SPHERICAL NANOPARTICLES FOR DETECTION AND THERAPY OF CANCER
}

A Dissertation Presented to the Faculty of the Graduate School at the University of Missouri

\author{
In Partial fulfillment of the Requirements for the Degree \\ Doctor of Philosophy
}

\section{By}

\section{DHANANJAY SURESH}

Dr. Raghuraman Kannan, Dissertation Advisor

December 2016 
(C) Copyright by Dhananjay Suresh 2016

All Rights Reserved 
The undersigned, appointed by the Dean of the Graduate School, have examined the dissertation entitled

CUBIC AND SPHERICAL NANOPARTICLES FOR DETECTION AND THERAPY OF CANCER

Presented by Dhananjay Suresh

A candidate for the degree of Doctor of Philosophy

And hereby certify that in their opinion it is worthy of acceptance.

Dr. Raghuraman Kannan

Department of Bioengineering

Department of Radiology

Dr. Liqun Gu

Department of Bioengineering

Dr. Sheila Grant

Department of Bioengineering

Dr. Anandhi Upendran

Department of Medical Pharmacology and Physiology

Institute for Clinical and Translational Science 
This work is dedicated to my family who gave me intellectual freedom, stood beside me and guided me. Special dedication to my grandparents.

$\&$

I also dedicate this work to the scientific and electronic arts that motivated me and gave ideas to complete this work. Thanks a lot iddqd and black mesa. 


\section{ACKNOWLEDGMENTS}

First, I would like to thank my PhD advisor, Dr. Raghuraman Kannan for his guidance, support and inspiration during the course of my research. Dr. Kannan gave me scientific freedom of thought that enabled a flow of ideas throughout my research. He corrected my scientific errors and gave me an impetus for learning the fundamentals in chemistry, molecular biology and materials research. This helped me to form a foundation, on which I devised my projects and investigated various aspects of natural and artificial sciences. Additionally, his cool demeanor made me relaxed at the hardest of times; thanks for putting up with me.

To my cool research group, for their unwavering support. Special thanks to Dr. Ajit without whom I could not have understood and completed my work with perfection. Your research experience helped solve all the scientific challenges I faced in the lab. To, Shreya Ghoshdastidar, Francisco Silva, Charles Caldwell and Nathan Applegren, who have been there since the beginning of my PhD. Our shared interests made life joyful, and our research discussions helped my deductive skills. These discussions enabled high quality science in my work. To, Dr. Soumavo Mukherjee, Dr. Abilash, and Sairam Yadavalli who were there to brainstorm and support my suggestions. Their frequent scrutiny improved my work. To, Kacey Ellwein, Jagjot Kainth, and Andrew Tarim who were always there to motivate and cheer me up. They made our lab awesome.

I would like to thank my committee members, Dr. Anandhi Upendran, Dr. Liqun Gu and Dr. Sheila Grant and for their valuable input in my research, support 
network and their professional advice for my career. They contributed novel ideas and supported my research work. In addition, their examination and questioning my work, further allowed me to investigate different aspects of the research data that I presented.

I would like to thank my collaborators, Dr. Vitaly Gruzdev, Jennifer Schnabel, Dr. Matthew Leevy, Sarah Chapman, Dr. Trupti Joshi, Dr. J. David Robertson, Dr. Henry White and Dr. Shubra Gangopadhyay for their personal time and involvement in my research work. They were always there to answer my questions and implement my research ideas in a practical setting.

To my friends, Sagar Gupta, Sachidevi Puttusamy, Ashutosh Surampalli, and Pratik Bhardwaj, whose liveliness provided me with an optimistic perspective of life.

Finally, to the University of Missouri that has been a great source of encouragement. It provided me a foundation for expressing my thoughts, brainstorming my ideas and conducting my interdisciplinary research in the field of Bioengineering, Nanotechnology, Photonics and Medicine. This has helped me to explore fundamental mechanisms regarding matter, energy and cellular behavior. These concepts will help us to understand nature and evolve our collective knowledge for a better future. 


\section{Table of Contents}

List of Figures

List of Schemes $\quad$ xx

List of Tables $\quad$ xxi

List of Equations $\quad$ xxii

$\begin{array}{ll}\text { List of Abbreviations } & \text { xxiii }\end{array}$

How targeted nanoparticles enter cancer cells?

$\begin{array}{ll}\text { CHAPTER } 1 & 4\end{array}$

$\begin{array}{lll}1.1 & \text { INTRODUCTION } & 4\end{array}$

$\begin{array}{lll}\text { 1.1.1 Current cancer therapy } & 4\end{array}$

1.1.2 Receptor mediated endocytosis 5

$\begin{array}{lll}\text { 1.1.3 Drug delivery vehicle } & 7\end{array}$

1.1.4 Molecular agents for targeting prostate cancer $\quad 8$

1.2 METHODS AND CHARACTERIZATION 9

1.2.1 Materials 9

$\begin{array}{lll}\text { 1.2.2 Instrumentation } & 10\end{array}$

1.2.3 Synthesis of Nanocages (NC1) 11

1.2.4 Synthesis of NC2 and NC3 12

1.2.5 Synthesis of NC5 and NC6 13

1.2.6 Characterization of Nanoconstructs 13

1.2.7 Peptide estimation 16

$\begin{array}{lll}1.2 .8 & \text { Cell Culture } & 20\end{array}$

1.2.9 Stability Studies 20

1.2.10 MTT Assay $\quad 22$

1.2.11 Estimation of $\mathrm{IC}_{50}$ in PC3 cells 23

1.2.12 Prevention of non-specific binding to glass substrates 25

1.2.13 Hyperspectral Imaging 26

1.2.14 Pathway Blocking Studies using endocytic inhibitors $\quad 27$

1.2.15 Antibody mediated co-localized inhibition 30

1.2.16 Image analysis for NC6 quantification in cells 30 
1.2.17 Quantification of nanoparticle uptake using Neutron Activation Analysis 32

1.2.18 TEM analysis for NC6 uptake in cells $\quad 35$

$\begin{array}{lll}\text { 1.2.19 } & \text { Statistical analysis } & 37\end{array}$

$\begin{array}{lll}1.3 & \text { RESULTS AND DISCUSSIONS } & 40\end{array}$

1.3.1 Properties of BBN conjugated gold nanocages 40

1.3.2 Receptor affinity of BBN conjugated gold nanocages 43

1.3.3 Mechanism of endocytosis $\quad 45$

1.3.4 Final fate of BBN conjugated gold nanocages in cells 50

1.3.5 Step (1) Pre-binding $\quad 51$

1.3.6 Step (2) Post-binding signaling cascade and (3) Membrane curving 52

1.3.7 Step (4) Clathrin pit formation and (5) Vesicular scission 53

1.3.8 Step (6) Endosomal/lysosomal accumulation and (7) Cytoplasmic release 55

$\begin{array}{lll}1.4 & \text { CONCLUSION } & 59\end{array}$

How targeted nanoparticles selectively detect circulating tumor cells (CTC) in blood? $\quad 60$

CHAPTER 2

$\begin{array}{lll}2.1 & \text { INTRODUCTION } & 62\end{array}$

2.1.1 Current diagnostic approaches $\quad 62$

2.1.2 Present detection technologies for Circulating tumor cells 64

2.1.3 Epithelial to mesenchymal transition $\quad 65$

2.1.4 Ultrasensitive detection using nanoparticles $\quad 67$

$\begin{array}{lll}2.2 & \text { METHODS AND CHARACTERIZATION }\end{array}$

$\begin{array}{lll}2.2 .1 & \text { Materials } & 67\end{array}$

$\begin{array}{lll}\text { 2.2.2 Instrumentation } & 68\end{array}$

2.2.3 Synthesis of Iron nanocubes $\quad 69$

2.2.4 Hydrophilic conversion of Iron nanocubes 70

2.2.5 Synthesis of pegylated nanocubes $\quad 71$

2.2.6 Antibody conjugation $\quad 72$

$\begin{array}{lll}2.2 .7 & \text { Characterization of nanoconjugates } & 72\end{array}$

$\begin{array}{lll}2.2 .8 & \text { ELISA } & 74\end{array}$

$\begin{array}{lll}\text { 2.2.9 SQUID magnetic analysis } & 75\end{array}$

2.2.10 Isolation of blood serum $\quad 77$

$\begin{array}{lll}\text { 2.2.11 Cell culture } & 77\end{array}$ 
$\begin{array}{lll}\text { 2.2.12 Capture methodology } & 77\end{array}$

$\begin{array}{lll}2.2 .13 & \text { EMT conversion } & 79\end{array}$

$\begin{array}{lll}2.2 .14 & \text { Western blot } & 79\end{array}$

$\begin{array}{lll}2.2 .15 & \text { Detection of cytokeratin } & 79\end{array}$

$\begin{array}{lll}2.2 .16 & \text { Statistics } & 80\end{array}$

2.3 RESULTS AND DISCUSSIONS $\quad 80$

2.3.1 Properties of FeNC constructs $\quad 80$

2.3.2 Specificity and Sensitivity Tests $\quad 84$

2.3.3 Quantification in blood serum 86

2.3.4 Investigating effect of EMT pathway on epithelial markers $\quad 87$

2.3.5 Detection of EMT status and EMT-CTCs 90

$\begin{array}{lll}2.4 & \text { CONCLUSION } & 93\end{array}$

How to structurally collapse nanoparticles for molecular release using external stimuli? 94 $\begin{array}{ll}\text { CHAPTER } 3 & 97\end{array}$

$\begin{array}{lll}3.1 & \text { INTRODUCTION } & 97\end{array}$

3.1.1 Smart nanomaterials for controlled drug delivery 98

$\begin{array}{ll}\text { 3.1.2 Localized surface plasmon resonance } & 100\end{array}$

3.1.3 Photon-electron-phonon (light-matter) interaction 102

3.1.4 Ultrafast shape assisted structural deformation 105

3.2 METHODS AND CHARACTERIZATION 106

$\begin{array}{lll}\text { 3.2.1 } & \text { Materials } & 106\end{array}$

$\begin{array}{lll}3.2 .2 & \text { Instrumentation } & 106\end{array}$

$\begin{array}{lll}\text { 3.2.3 Synthesis of cubic nanoparticles } & 107\end{array}$

$\begin{array}{lll}3.2 .4 & \text { Characterization } & 109\end{array}$

3.2.5 Laser setup and real-time spectroscopy 110

3.2.6 Exposure of nanoparticles 114

$\begin{array}{lll}3.2 .7 & \text { Cell culture } & 115\end{array}$

$\begin{array}{lll}3.2 .8 & \text { Cell viability tests } & 115\end{array}$

$\begin{array}{lll}3.2 .9 & \text { FDTD simulation } & 117\end{array}$

3.2.10 Single nanoparticle spectroscopy 119

3.2.11 HR-TEM STEM and EDX analysis 119

3.2.12 Atomic force microscopy 122 
3.2.13 Size distribution analysis 122

3.2.14 Image analysis using TEM 123

$\begin{array}{lll}3.2 .15 & \text { Statistics } & 123\end{array}$

3.3 RESULTS AND DISCUSSIONS 123

3.3.1 Properties of different nanocubic architectures 123

3.3.2 Simulating nanoplasmonic field enhancements 125

3.3.3 Activation thresholds of cubic nanoparticles 130

3.3.4 Controlled particle modifications 132

3.3.5 Characterization of single nanoparticle deformations 138

3.3.6 Lattice rearrangement in single nanoparticles 143

3.3.7 Mechanism of excitation in cubic nanoarchitectures 148

$\begin{array}{lll}3.4 & \text { CONCLUSION } & 152\end{array}$

How to design nanoparticles to aid in reversing drug resistant cancer using internal stimuli?153 $\begin{array}{ll}\text { CHAPTER } 4 & 155\end{array}$

$\begin{array}{lll}4.1 & 155\end{array}$

4.1.1 Lung cancer diagnosis and treatment 156

$\begin{array}{lll}\text { 4.1.2 } & \text { Acquired drug resistance to TKI } & 157\end{array}$

4.1.3 Role of AXL in TKI resistance 159

$\begin{array}{lll}\text { 4.1.4 AXL regulation of EMT } & 160\end{array}$

4.1.5 Inhibition of AXL using siRNA (inhibitor therapy) 161

4.1.6 Inhibition of AXL using crRNA (gene therapy) 162

4.1.7 Translational medicine: Delivery of siRNA using nanoparticles 164

$\begin{array}{lll}4.2 & \text { METHODS AND CHARACTERIZATION } & 168\end{array}$

$\begin{array}{lll}\text { 4.2.1 } & \text { Materials } & 168\end{array}$

$\begin{array}{lll}\text { 4.2.2 Instrumentation } & 170\end{array}$

4.2.3 siRNA and crRNA agents $\quad 171$

4.2.4 Synthesis of Gelatin Nanoparticles 171

4.2.5 Conjugation of antibody and siRNA 172

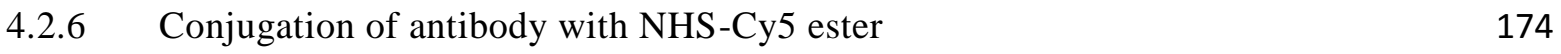

4.2.7 Characterization of nanoconstructs 174

$\begin{array}{lll}\text { 4.2.8 Cell culture } & 175\end{array}$

$\begin{array}{lll}\text { 4.2.9 } & \text { Fluorescence microscopy } & 176\end{array}$ 
4.2.10 CRISPR for gene knockout cells 176

4.2.11 mRNA and miRNA expression analysis using RT-PCR 177

$\begin{array}{lll}\text { 4.2.12 Western blot } & 178\end{array}$

$\begin{array}{lll}\text { 4.2.13 Invasion and migration assay } & 179\end{array}$

4.2.14 Gelatin zymography 180

4.2.15 Apoptosis cell-cycle analysis 180

4.2.16 MTT viability assay 181

4.2.17 Generation of A549 luciferase tumor xenografts 182

4.2.18 Mice treatment protocol 183

4.2.19 Tumor imaging and analysis 183

$\begin{array}{lll}\text { 4.2.20 Statistics } & 184\end{array}$

$\begin{array}{lll}4.3 & \text { RESULTS AND DISCUSSIONS } & 184\end{array}$

$\begin{array}{lll}\text { 4.3.1 Nanoparticle synthesis and characterization } & 184\end{array}$

$\begin{array}{ll}\text { 4.3.2 Nanoparticle targeting and delivery } & 188\end{array}$

4.3.3 Downregulation of AXL using siRNA and generation of knockout cell line 190

4.3.4 mRNA and miRNA expression of different oncogenic factors towards AXL 195

4.3.5 AXL regulates MMP-2 and MMP-9 in drug resistant NSCLC 201

4.3.6 AXL knockdown deregulates mTOR pathway in NSCLC 206

4.3.7 AXL crosstalk with FN14 may be independent of EGFR mutant status 210

4.3.8 Mechanism of AXL-FN14 and its role in TKI resistance 211

4.3.9 Targeting AXL-FN14 using nanoparticles in vitro 222

4.3.10 Targeting AXL-FN14 using nanoparticles in A549 mice xenografts 224

$\begin{array}{lll}\text { 4.3.11 Impact of gene therapy in cancer } & 227\end{array}$

$\begin{array}{lll}4.4 & \text { CONCLUSION } & 229\end{array}$

$\begin{array}{ll}\text { IMPLICATIONS OF THESE STUDIES } & 230\end{array}$

$\begin{array}{ll}\text { REFERENCES } & 231\end{array}$

$\begin{array}{ll}\text { Vita } & 260\end{array}$ 


\section{List of Figures}

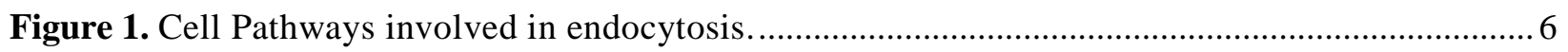

Figure 2. UV-vis absorption spectrum for silver nanocubes ......................................................... 14

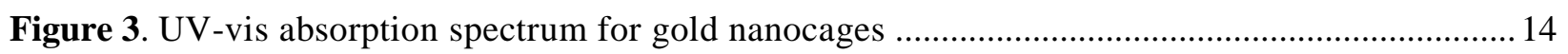

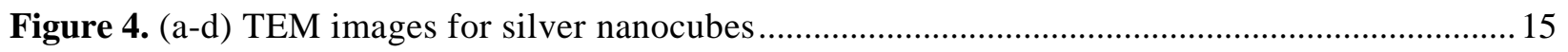

Figure 5. (a-d) TEM images for gold nanocages .......................................................................... 15

Figure 6. (a) Size distribution indicates AuNCs synthesized are of uniform size (b) Shape dispersity

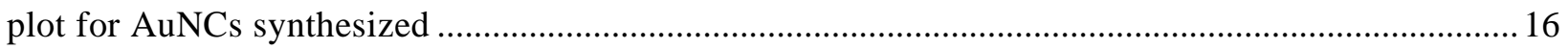

Figure 7. UV-visible spectroscopy studies using SS-BBN peptide. SS-BBN peptide was treated with $\mathrm{NaCN}$ and spectra was recorded at different wavelengths to ensure the structural integrity of the peptide. (a) SS-BBN peak at $280 \mathrm{~nm}$ does not shift in presence of $\mathrm{NaCN}$ solution. (b) No absorption in the NIR region

Figure 8. Estimation of bombesin using HPLC. (a) Retention curves of SS-BBN peptide in different concentrations; (b) 3rd order polynomial standard curve constructed using area under curves of SS $\mathrm{BBN}$ at different concentration levels. (Detector wavelength: $280 \mathrm{~nm}$; Column flow rate $1.0 \mathrm{ml} / \mathrm{min}$ with $20 \mu$ l volume of injection)

Figure 9. (a) UV-Visible spectra and (b) TEM images of NC3 and NC6. NC3 and NC6 are bombesin conjugated gold nanocages. Plasmon absorption of both nanoconstructs at NIR region indicates the size and shape similarity.

Figure 10. UV-visible spectra for $\mathrm{NC} 3$ or $\mathrm{NC} 1$ at different wavelength ranges before and after $\mathrm{NaCN}$ digestion. (a) NC3 sample showed absorption band at $280 \mathrm{~nm}$ corresponding to BBN peptide, whereas no corresponding peak was observed for NC1. (b) After digestion, plasmon absorption band for AuNC disappears .18

Figure 11. UV-Visible spectra for NC6 or NC4 at different wavelength ranges before and after NaCN digestion. (a) NC6 sample showed absorption band at $280 \mathrm{~nm}$ corresponding to BBN peptide, whereas no corresponding peak was observed for NC4. (b) After digestion, plasmon absorption band for AuNC disappears .19

Figure 12. Estimation of bombesin using UV-visible spectroscopy and HPLC. (a) UV-visible spectra for digested and centrifuged NC3 and NC6 samples (dry powder); (b) HPLC retention curves for digested and centrifuged NC3 and NC6 samples (dry powder). Results from UV-visible spectroscopy corroborate with that of HPLC data. (HPLC: Detector wavelength: $280 \mathrm{~nm}$; Column flow rate 1.0 $\mathrm{ml} / \mathrm{min}$ with $20 \mu \mathrm{l}$ volume of injection) ...... 19 
Figure 13. Estimation of bombesin using UV-visible spectroscopy. (a) UV-visible spectra of SS-BBN peptide in different concentrations; (b) 3rd order polynomial standard curve constructed using absorption of SS-BBN at different concentration levels. 20

Figure 14. Stability of NC3 in different biologically relevant solutions with time was monitored using UV-visible spectroscopy. NC3 was treated with different solutions and plasmon absorption band monitored at (a) $1 \mathrm{~h}$ (b) $24 \mathrm{~h}$. Results indicate that nanocages are very stable even after $24 \mathrm{~h}$. However, aggregated cages were visibly observed in the vials. 21

Figure 15. Stability of NC6 in different biologically relevant solutions with time was monitored using UV-visible spectroscopy. NC6 was treated with different solutions and plasmon absorption band monitored at (a) $1 \mathrm{~h}$ (b) $24 \mathrm{~h}$. Results indicate that nanocages are very stable even after $24 \mathrm{~h}$. No visible aggregation was observed as PEG coating improves the stability significantly. 21

Figure 16. Stability of NC6 in different biologically relevant solutions with time was monitored using UV-visible spectroscopy. NC6 was treated with different solutions and plasmon absorption band

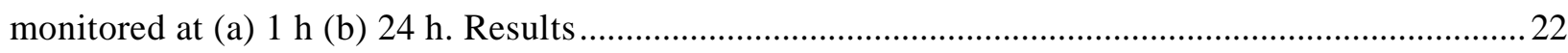

Figure 17. $\mathrm{IC}_{50}$ dosage response curves for nanoconjugates NC2, NC3, NC5 and NC6...................24

Figure 18. Non-specific binding of gold nanocages on the substrate was reduced by pretreatment with charged aminoacid homopolymers. (a-b) Prior to poly-L-lysine treatment, nanocages were seen everywhere on the glass slide along with PC3 cells; (c-d) After poly-L-lysine treatment, non-specific binding of nanocages to glass slide is visibly reduced. These images show poly-L-lysine coating on the substrate reduced non-specific binding of AuNCs. .25

Figure 19. Construction of hyperspectral library for AuNC-BBN. (a) Dark field fluorescence image for bombesin conjugated AuNCs internalized in PC3 cells; (b) Corresponding hyperspectral image; (c) Spectral plot for a single Bombesin conjugated AuNC spot on a glass slide; (d) Spectral plot for a single Bombesin conjugated AuNC spot internalized within PC3 cells; (e) Hyperspectral library for Bombesin conjugated AuNCs coated on a glass slide with corresponding hyperspectral image; (f) Hyperspectral library for bombesin conjugated AuNCs internalized in PC3 cells Hyperspectral profile match confirms AuNC-BBN signals. Hyperspectral imaging of AuNC-BBN was not influenced in

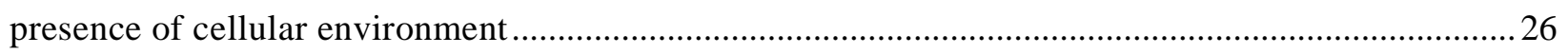

Figure 20. Dark field images of PC3 cells treated with nanoconjugates (a) NC3 after treatment with cells at $37^{\circ} \mathrm{C}$; (b) NC6 after treatment with cells at $37^{\circ} \mathrm{C}$; (c) NC3 after treatment with cells at $4{ }^{\circ} \mathrm{C}$ (d) NC6 after treatment with cells at $4{ }^{\circ} \mathrm{C}$. At $4{ }^{\circ} \mathrm{C}$ no internalization was observed as all endocytic processes were arrested. This result shows that nanocage internalization is energy dependent. .........28 Figure 21. Dark field imaging for different type of surface coated nanoconjugates incubated with PC3 cells, with specific endocytic pathways blocked using inhibitors. Top panel: Caveolae pathway blocked 
(a) NC1 on live cells (b) NC4 on live cells (c) NC3 on live cells (d) NC6 on live cells; Bottom panel: Clathrin pathway blocked (e) NC1 on live cells (f) NC4 on live cells (g) NC3 on live cells (h) NC6 on live cells. This result confirms that internalization of bombesin conjugated gold nanocages is dependent on clathrin pathway. .29

Figure 22. Glossary for schematics showing 2-D representation of the cell membrane during CME. 33 Figure 23. Flow cytometry dot plots showing FSC and SSC. For cell samples, R2 represents gate to sort PC3 cells from total registered events. Nanocage events falling outside the gate (based on control FCS and SSC for NC6) are excluded from sort. R1 represents gate to sort live cell events negative for 7AAD into collection tube. (a-b) NC6 in media for control plot; (c-f) UNT: Duplicate experiments for NC6 treated PC3 cells pretreated with no inhibitors; (g-j) AB: Duplicate experiments for NC6 treated PC3 cells pretreated with X-22 anti-clathrin antibody; (k-n) Duplicate experiments for NC6 treated PC3 cells pretreated with clathrin pathway inhibitor chlorpromazine. 35

Figure 24. TEM images showing plasma membrane of PC 3 cells after exposure to bombesin conjugated gold nanocages. (a-f) Clathrin coated pit formation associated with endocytosis of NC6. .36 Figure 25. TEM images showing plasma membrane of PC3 cells after exposure to bombesin conjugated gold nanocages. (a-f) The transition of invaginations to form budded clathrin coated vesicles in the cytoplasm was observed. .36

Figure 26. TEM images showing trafficking of AuNC-BBN into endosomal vesicles and lysosomes. (a) NC6 still attached to the GRP receptor on the inner side of clathrin coated vesicle; (b-c) AuNCs final fate in lysosomes. AuNCs exit the lysosomes and enter into cytoplasmic cellular space. .37

Figure 27. Statistical analysis on internalization of NC6 in PC3 cells in presence of inhibitors..........38 Figure 28. TEM images of nanoconjugates stained with uranyl acetate. (a) NC1; (b) NC3; (c) NC4; (d) NC6. TEM grids were stained immediately after deposition to minimize surface coat contraction due to solvent dissipation. Blue arrow indicates diagonal core length of particles, yellow arrow indicates total hydrodynamic size and red arrow indicates thickness. Results show presence of surface $\mathrm{PVP} / \mathrm{PEG} / \mathrm{Bombesin}$ coating over the nanocages. .43

Figure 29. PC3 cells were fixed and treated with constructs. Nanoparticles were allowed to interact and bind with the GRP receptors still present on the cell surface. Bombesin conjugated AuNCs recognize these receptors on the cell surface and bind to them. Representative dark field backscattering images of fixed PC3 cells after treatment with the following nanoconjugates: $(\mathrm{a}-\mathrm{c}) \mathrm{NC} 1$; (d-f) NC4; (g-i) NC3; and (j-1) NC6. 100X oil.

Figure 30. Bombesin conjugated nanocage NC6 requires dynamin for entry in PC3 cells. NC6 was treated with PC 3 cells in the presence and absence of a dynamin inhibitor. Representative dark field 
images (stained with DAPI and CellMask; 100x oil) of cells in the (a-c) absence and (d-f) presence of dynamin inhibitor.

Figure 31. Caveolae inhibitors $M \beta C D$ and filipin did not influence the internalization of NC6 in PC3 Cells, whereas clathrin inhibitors anti-clathrin antibody, chlorpromazine and pitstop-2 significantly influence the internalization. Representative dark field images (stained with DAPI and CellMask; 100x oil) of cells treated with caveolae inhibitors (a-c) M $\beta C D$; (d-f) Filipin; and clathrin inhibitors (g-i) X22 anti-clathrin antibody; (j-1) Chlorpromazine; and (m-o) Pitstop-2. .48

Figure 32. Quantitative analysis of NC6 internalized in PC3 cells in the presence and absence of caveolae and clathrin inhibitors using optical microscopy technique (dark field fluorescence illumination). The legend indicates the following: (UNT) untreated; (DYN) dynamin pathway blocked with Dynasore; (CAV-M $\beta C D)$ caveolae pathway blocked with methyl- $\beta$-cyclodextrin; (CAV-FIL) caveolae pathway blocked with filipin; (CLT-X22AB) clathrin pathway inhibited during colocalization by anti-clathrin antibody; (CLT-CLOR) clathrin pathway blocked with chlorpromazine; (CLT-PIT2) clathrin pathway blocked by Pitstop-2. The nanoparticles in two hundred cells were monitored and quantified. Each visible gold signal, i.e., nanoaggregate, was approximated as one unit. PC3 cells treated with clathrin inhibitors internalize fewer AuNCs. ${ }^{\star \star} \mathrm{p} \leq 0.01$, and ${ }^{\star \star \star} \mathrm{p} \leq 0.001$ and the values were analyzed using one-way anova.

Figure 33. Quantitative analysis of NC6 internalized in PC3 cells in the presence and absence of clathrin inhibitors using NAA. The legend indicates the following: (UNT) untreated; (CLT-X22AB) clathrin pathway blocked with Antibody; (CLT-CLOR) clathrin pathway blocked with chlorpromazine. ${ }^{\star} \mathrm{p} \leq 0.05$, and the values were analyzed using one-way anova. .50

Figure 34. (a, b) TEM images of NC6 in a prebinding cellular environment (see arrows). (c) Schematic representation of the prebinding cellular environment. (Molecules depicted in all subsequent steps can be identified using a glossary available in the Supporting Information; Figure S28.)

Figure 35a. (a, b) Binding of NC6 with GRP receptors on PC3 cancer cells is shown in TEM images. The TEM images show postbinding signal cascade and membrane intrusion environment within cells (see arrows). (c) Schematic representation of the above environment depicting the important events 52 Figure 36a. Formation of clathrin coated pits in the cellular membrane is characteristic of clathrin mediated endocytosis of NC6. $(a, b)$ Before internalization of nanocage the membrane pit formation is observed in TEM images along the cellular membrane (labeled with arrows). (c) Schematic representation of the primary invagination and clathrin cage formation (scaffolding complexes lining the pit are now shown as a blue dotted line for simplicity). .54 Figure 37. Trafficking of nanocages within cells is controlled by the method of endocytosis. NC6 conjugates were internalized via CME process. $(a, b)$ TEM images show uncoating of clathrin coated 
vesicles (labeled with arrow) and their transport within cells as early endosomes. (c) Schematic representation of uncoating and vesicular transport of nascent internalized vesicle. .56

Figure 38. Release of nanocages from lysosome is an important event in trafficking. (a,b) TEM images showing NC6 unbinding within lysosomes. The arrows in the figure indicate AuNCs present in cytoplasm after their eventual lysosomal release. (c) Schematic representation of the endosomal receptor recovery and lysosomal degradation process. .57

Figure 39. TEM analysis of NC6 internalized in PC3 cells in the presence and absence of caveolae and clathrin inhibitors. The legend indicates the follows: (UNT) Untreated; (CAV-FIL) Caveolae pathway blocked with Filipin; (CLT-X22AB) Clathrin pathway inhibited during co-localization by anti-clathrin antibody; (CLT-CLOR) Clathrin pathway blocked with Chlorpromazine. (a, d, g, and j) Representative images for internalization; (b, e, h and k) Representative images for endosomal accumulation; (c, f, i and 1) Representative images for cell morphology. Arrows denote nanocages....................................58

Figure 40. Tumor shedding caused by EMT upregulation in tumor cells. .65

Figure 41. TEM imaging for (a) FeNC coated with polymer and (b) FeNC after pegylation. The cubic particles were uniform with an edge length of $20 \mathrm{~nm}$. .73

Figure 42. Magnetization vs temperature curve for FeNCs measured at zero field cooled state at 1000 Oe.

Figure 43. Overlay of magnetization vs temperature curve for FeNCs measured at zero field cooled state and field cooled warm state at 1000 Oe. The blocking temperature was calculated to be -163.15 ${ }^{\circ} \mathrm{C}$.

Figure 44. Magnetic hysteresis curve of FeNC obtained from SQUID measurements made at 2 K...81

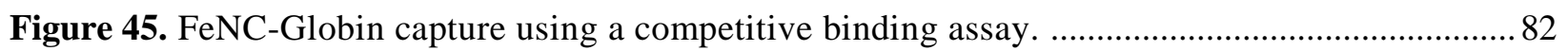

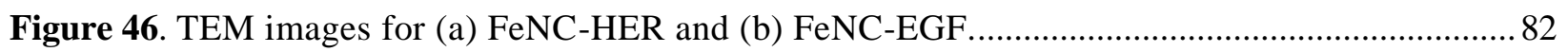

Figure 47. Properties of the external field applied for FeNC-CTC capture process........................... 83

Figure 48. Cells were stained with (a) red dye using CellMask and (b) blue dye using Hoechst........ 84

Figure 49. Western blot imaging shows that A549 cells were positive for HER-2, while HCC827 were not. Further the blot show that both cells were positive to EGFR, with HCC827 being more than A549.

Figure 50. Specificity of (a) FeNC-HER and (b) FeNC-EGF towards HER-2 (A549) and EGFR (Hcc827/A549) expressing cells as function of surface antibody loading. .85

Figure 51. Sensitivity of FeNC-HER and FeNC-EGF towards HER-2 (A549) and EGFR (Hcc827/A549) expressing cells at different particle concentrations (a) $1 \times 10^{6}$ and (b) $2 \times 10^{6}$. .86 
Figure 52. Cell capture from A549-HCC827 mixture spiked in serum using FeNC-HER and FeNCEGF constructs. FeNC was used as a control to study non-specific binding. For this study (a) $10^{4}$, (b) $10^{3}$, (c) $10^{2}$ cells were spiked and captured using FeNC-ab constructs.

Figure 53. Microscopy for EMT activated cells indicate an elongated elastic morphology with reduced surface binding signifying loss of epithelial binding markers used for adhesion (40x; bright field) .. 88 Figure 54. Western blotting for analysis of EMT marker Vimentin, epithelial markers EpCAM and cytokeratin, and oncogenic overexpressed markers EGF and HER-2 in normal A549 and EMT activated A549 lung cancer cells. .89

Figure 55. EMT-cells captured by (a) FeNC-HER and (b) FeNC-EGF show decreased epithelial cytokeratin expression. 91

Figure 56. Amount of cells captured by FeNC-EGF (a) before and (b) after EMT. Results indiciate FeNC-EGF can capture as fewer as 10 cells without any loss in sensitivity after EMT. ${ }^{\star \star} \mathrm{p} \leq 0.01$, and the values were analyzed using one-way anova.

Figure 57. Amount of cells captured by FeNC-HER (a) before and (b) after EMT. Results indiciate FeNC-HER can capture as fewer as 10 cells without any loss in sensitivity after EMT. ${ }^{\star} \mathrm{p} \leq 0.05$, and the values were analyzed using one-way anova.

Figure 58. TEM images for (a) solid Ag nanocubes - NC, (b) Au-Ag nanoboxes with small cavity NB, (c) hollow Au-Ag nanocages - CG and (d) Au nanoframes - NF. 109

Figure 59. Fundamental wavelength of the Femtosecond pulse. 110

Figure 60. Reduced wavelength of the Femtosecond pulse at $386 \mathrm{~nm}$. 111

Figure 61. Custom laser setup for the experiments. 111

Figure 62. Sensors S1, S2 and S3 for measuring transmitted light within the setup. 113

Figure 63. 96 well plates were exposed using a stage controller and focusing mirror. 116 Figure 64. Femtosecond laser pulses at the NIR region did not affect cell viability. At the highest duration, both low and high power setting showed a viability of $85-90 \%$. Results are from triplicates. 116

Figure 65. Femtosecond laser pulses at the NIR region did not affect cell viability after CG internalization. At the highest concentration, both low and high power setting showed a viability of 75-80\%. Results are from triplicates.

Figure 66. Scattering simulation for $\mathrm{Ag}$ and $\mathrm{Au}$ spheres. (a) $50 \mathrm{~nm} \mathrm{Ag}$ sphere, (b) Ag sphere with a dielectric layer, (c) $50 \mathrm{~nm}$ Au sphere, and (d) Au sphere with dielectric layer. 118

Figure 67. Crystallography for solid Ag nanocubes indicates a crystalline structure. 120 Figure 68. Crystallography for porous particles: (a) nanoboxes and (b) nanocages, indicate a polycrystalline structure. 120 
Figure 69. Crystallography for fragmented particles indicate a polycrystalline structure. 121

Figure 70. Crystallography for melted spheres indicate a crystalline structure. 121

Figure 71. Au-Ag alloy composition for (a) Nanoboxes and (b) Nanocages. Results indicate the crystal structure remains preserved after laser treatment.

Figure 72. UV-vis absorbance profiles for (a) Nanocubes (b) Nanoboxes and (c) Nanocages, before and after exposure. BE, ST, DT and MT stand for Before exposure, Spectral threshold, Deformation threshold and Melting threshold respectively.

Figure 73. Simulated near-field E-field scattering for (a) Nanocubes (b) Nanoboxes and (c) Nanocages. 126

Figure 74. Simulated total E-field for (a) Nanocubes (b) Nanoboxes and (c) Nanocages for top and center cross sections for each particle structure. 126

Figure 75. Simulated power absorbance profiles for (a) Nanocubes (b) Nanoboxes and (c) Nanocages for top and center cross sections for each particle structure.

Figure 76. Simulated near-field E-field scattering for (a) silver spheres and (b) gold spheres. 128

Figure 77. Simulated total E-field for (a) silver spheres and (b) gold spheres for top and center cross sections for each particle structure.

Figure 78. Simulated power absorbance profiles for (a) silver spheres and (b) gold spheres for top and center cross sections for each particle structure.

Figure 79. Activation thresholds for (a) Nanocubes (NC) (b) Nanoboxes (NB) and (c) Nanocages (CG) using linear ( $\mathrm{Li}$ ) and circular $(\mathrm{Ci})$ polarization exposure by femtosecond light pulses. Results indicate discrete levels of interaction energies for the three particles. ${ }^{\star \star} \mathrm{p} \leq 0.01$, and ${ }^{\star \star \star} \mathrm{p} \leq 0.001$ and the values were analyzed using one-way anova

Figure 80. No particles modifications were observed in CGs $(710 \mathrm{~nm})$ when the laser wavelength was detuned to $400 \mathrm{~nm}$ at both (a) medium; 20-80 $\mu \mathrm{J}$ and (b) high power; (> $90 \mu \mathrm{J}$ ).

Figure 81. No particles modifications were observed in (a) Nanocubes, (b) Nanoboxes and (c) Nanocages at spectral threshold (ST) 133

Figure 82. (a) Particle modification stages during the course of exposure in nanocubes (NC), nanoboxes (NB) and nanocages (CG). Results show deformation threshold (DT) (in between spectral threshold (ST) and melting threshold (MT)) specifically modifies particles at their plasmonic hot-spot locations at corners or cavities. (b) associated Lattice displacements in NCs at DT (c) Corner and edge fragmentations in CGs at DT (d) localized cavity enlargement in NBs to form ultrashort pulse assisted CGs at DT. 135

Figure 83. (a) Gradual shift in UV-vis absorption profile for CG formation using NB using wet chemical synthesis (b) Formation of CGs from NBs using laser pulses. (Top-bottom) Graphs represent 
average size as a measure of edge length, transformation statistics and cavity profile at various incident energies. The last column in all graph represent CG statics from wet synthesis for comparison. ${ }^{\star \star \star} \mathrm{p} \leq$ 0.001 and the values were analyzed using one-way anova 137

Figure 84. Particle size distribution profiles for (a) nanocubes, (b) nanoboxes, (c) nanocages and (d) comparison with as synthesized nanoframes. BE, ST, DT and MT stand for Before exposure, Spectral threshold, Deformation threshold and Melting threshold respectively. 139

Figure 85. AFM surface topography profile for a nanobeam formed during nanocage fragmentation revels asymmetrical bending and flattening of structures. 139

Figure 86. Rayleigh scattering of light by (a) NCs, (b) NBs, and (c) CGs (dark-field, 100x oil) ..... 140 Figure 87. Rayleigh scattering of single nanoparticles (a) NCs, (b) NBs, and (c) CGs. BE, ST, DT and MT stand for Before exposure, Spectral threshold, Deformation threshold and Melting threshold respectively.

Figure 88. Scattering simulation for (a) NCs, (b) NBs, and (c) CGs. Results show a similar peak for NCs at $450 \mathrm{~nm}$, double peak for NBs at $420 \mathrm{~nm}$ and $580 \mathrm{~nm}$, and a $700 \mathrm{~nm}$ peak for CGs. 141

Figure 89. Rayleigh scattering of single nanoframes. BE and MT stand for Before exposure and Melting threshold respectively.

Figure 90. Computed 3D Near-field enhancements for (a) NC, (b) NB and (c) CG. Results clearly show selective lasing in cavities of NB as compared to NC and CG.

Figure 91. STEM-HAADF images for (a) deformed NC and (b) expanded view of the particle showing uniform crystalline lattice structure. 143

Figure 92. STEM-HAADF images for (a) melted spherical NC and (b) expanded view of the particle showing uniformly rearranged crystalline lattice structure. 144

Figure 93. STEM-HAADF images for (a) modified NB (b) modified NB (c) an opened CG and its associated (d-f) bended joints. 144

Figure 94. STEM-HAADF images for (a) unexposed nanocage $(\mathrm{CG})$ with the (b) expanded view showing a uniform polycrystalline lattice (c) a deformed CG with its expanded views showing (d) uniform atomic columns of mixed $\mathrm{Au}$ and $\mathrm{Ag}$ atoms similar to unexposed $\mathrm{CG}$ and (e) similar structure at the fragmented corner indicating a columbic explosion or electrostatic repulsion at play. 145 Figure 95. STEM-EDX analysis for a modified NB: (a) Thick gold exterior (b) a dispersed Ag interior (c) an alloyed composition for the particle. 146

Figure 96. STEM-EDX analysis for a (a) spherical particle indicates (b) silver composition devoid of gold. 146

Figure 97. TEM images (a-i) show presence of amorphized PVP jets at the surface of modified particles exposed at DT. 147 
Figure 98. TEM images showing melted aggregated networks formed at MT (a) melted NC (b) melted CG. . 148

Figure 99. Activation thresholds for NC, NB, CG using linear (Li) and circular (Ci) polarization exposure by femtosecond light pulses in three different mediums of varied thermal conductivities. Results indicate an increase in required activation energy with increasing thermal conductivities. * indicate $\mathrm{p} \leq 0.05,^{\star \star}$ indicate $\mathrm{p} \leq 0.01$, and ${ }^{\star \star \star}$ indicate $\mathrm{p} \leq 0.001$ and the values were analyzed using one-way anova 149

Figure 100. Transmittance spectroscopy of (a) Bombesin peptide as control showing a peak at $220 \mathrm{~nm}$ and (b) CG-bombesin that was exposed at DT showing a peak at $245 \mathrm{~nm}$ suggesting release of peptide. 151

Figure 101. Activation of RISC complex using siRNA to degrade AXL mRNA in the cytoplasm. . 162 Figure 102. Formation of indel in the AXL gene using the complex formed by CRISPR RNA, Tracer guide RNA and the Cas9 endonuclease protein.

Figure 103. The three components needed to target, release drug and destroy resistance causing oncogenic mRNA within cell cytoplasm. 166

Figure 104. Design of an antibody conjugated porous drug encapsulated multifunctional nanoparticle with siRNA on its surface. These particles once internalized will degrade within the cytoplasm using proteases and release both drug and siRNA to block and inhibit EGFR pathway and AXL pathway repectively. 167

Figure 105. Standard curves for (a) Ab-Cy5 using Cetuximab and (b) siAXL-Cy5. 175 Figure 106. TEM images for (a) Gelatin nanoparticle (G) (b) antibody conjugated GAb and (c) antibody and siRNA conjugated GAbsiAXL. 185

Figure 107a. Stability of Gelatin nanoparticle constructs evaluated through change in surface potential over time. 186

Figure 108. Internalization of targeted constructs in (a) H820 and (b) HCC827 cells (40x). Results indicate gelatin particles (Fluorescein) are only internalized when antibody is present on surface. Similarly, siRNA is internalized when gelatin particles are internalized by antibody targeting. ....... 188 Figure 109. Internalization of targeted constructs in HCC827 cells: (a) GAb (b) GabsiAXL-Cy5 (60x). Results indicate presence of high quantities of siRNA (Cy5) in the cytoplasm of cells after internalization of construct.

Figure 110. Downregulation of AXL using siRNA in H820 cells: (a) Band densitometry analysis (b) associated blot.

Figure 111. Downregulation of AXL using siRNA conjugated constructs in H820 cells: (a) associated blot (b) Band densitometry analysis. Downregulation of FN14 using siRNA conjugated constructs in 
H820 cells: (a) associated blot (b) Band densitometry analysis. MP and OP stand for Modified protocol and Old protocol respectively. Gq and Gnq stand for gelatin particles with glutaraldehyde quenched and non-quenched respectively.

Figure 112. Downregulation of AXL using siRNA conjugated constructs in H820 cells: (a) associated blot (b) Band densitometry analysis. Results indicate that physical mixture of GAb and siRNA does not allow downregulation. Results also show that storing lyophilized power at $-20{ }^{\circ} \mathrm{C}$ does not adversely affect function of construct.

Figure 113. Cumulative analysis of AXL downregulation using siRNA alone, siRNA conjugated constructs and synergistic treatment of GAbsiAXL with erlotinib in H820 cells. Results show siRNA was efficiently downregulating AXL when present in sample. These results suggest nanoparticle based delivery of siRNA was highly stable and efficient. As shown in figure GAb control did not affect AXL in cells. ${ }^{\star \star \star} \mathrm{p} \leq 0.001$, and the values were analyzed using one-way anova

Figure 114. Protein analysis between H820 and CRISPR generated AXL knockout H820AK cells: (a) associated blot and (b) Band densitometry analysis for blot and (c) Representative heat map showing relative expressions $($ Red=high; Blue=low)..... 195

Figure 115. RT-PCR gene expression analysis for (a) AXL, (b) P53, (c) EGFR, and (d) MZF1. Results were averaged from 3 independent experiments.

Figure 116. Apoptosis assay analysis for (a) Late-stage at $48 \mathrm{~h}$ and (b) Early-stage at $72 \mathrm{~h}$ shows relative increase during synergistic treatment of siRNA based samples with erlotinib in H820 cells. (c) Associative flow cytometry data for apoptosis results. Q2 and Q4 indicate late and early-stage apoptosis respectively. Results were averaged from 2 independent experiments. 198

Figure 117. RT-PCR micro-RNA expression analysis for (a) miR-34a, (b) miR-432, (c) miR-548b, and (d) miR-374. Results were averaged from 3 independent experiments.

Figure 118. Proliferation analysis in H820 cells using (a) Invasion assay and (b) Migration assay. 201 Figure 119. Zymogram analysis in H820 cells for MMP-2 and MMP-9 activity using conditioned media: (a) Associated zymograph (b) Band densitometry analysis. Results were averaged from 3 independent experiments. ${ }^{\star \star \star} \mathrm{p} \leq 0.001$, and the values were analyzed using one-way anova.........203 Figure 120. Western blot for AXL siRNA based treatments, controls and synergistic treatment with erlotinib in H820 cells: (a) EMT pathway (b) mTOR-AKT axis and apoptosis pathway and (c) EGFR and associated survival pathways. 204

Figure 121. Band densitometry analysis for associated blots shown in Figure 120. Data represents protein analysis for AXL siRNA based treatments, controls and synergistic treatment with erlotinib in H820 cells. 205 
Figure 122. Representative heat map for data shown in Figure 121 (Red=high; Blue=low). Data represents protein analysis for AXL siRNA based treatments, controls and synergistic treatment with erlotinib in H820 cells. Results were averaged from 2 independent experiments. ........................... 206 Figure 123. Relative expression of FN14 when AXL is downregulated in H820 cells. Results were averaged from 3 independent experiments. ${ }^{\star \star \star} \mathrm{p} \leq 0.001$, and the values were analyzed using one-way anova. 210

Figure 124. Relative expression of FN14 when AXL is downregulated in NSCLC cells: (a) associated western blot and (b) Band densitometry analysis for A549 cells showing FN14 upregulation. 211 Figure 125. (a) Western blot for AXL-FN14 survival cross-talk analysis in H820 cells using siRNA and dual siRNA-construct, GAbsi(AXL,FN14) treatments (b) associated band densitometry analysis.

Figure 126. Representative heat map for data shown in Figure 125 (Red=high; Blue=low). Data represents protein analysis for AXL and FN14 siRNA based treatments in H820 cells 214

Figure 127. AXL and FN14 weighed gene network analysis: (a) Biochemical pathway association and (b) Downstream network association. 215

Figure 128. Micro-RNA association heat map based on experimental PCR data in H820 cells. (White=high; Blue=low). Data represents several miRNAs that scored for high associations with AXL, P53, EGFR and MZF1 out of 153 screened miRNAs. .216

Figure 129. KEGG gene association heat map based on experimental PCR and protein data for lung cancer cells. (White=high; Blue=low). Data shows A549 and many other cells lines may high associative scores for AXL and FN14. 217

Figure 130. 72 h Cell viability analysis of (a) DMSO and Transit-X2 in H820, (b) Cetuximab antibody in H820 and HCC827 (c) siRNA in H820, (d) siRNA in H1975, and (e) siRNA in A549 cells........219

Figure 131. $72 \mathrm{~h}$ Toxicity of erlotinib in various NSCLC cells lines: (a) Cell viability plot and (b) $\mathrm{IC}_{50}$ analysis. Results were averaged from 3 independent experiments.

Figure 132. Resensitization to erlotinib in various NSCLC cells lines by blocking AXL/FN14/KRAS pathways in H820, H1975 and A549 NSCLC cells for 72 h. (a, c, e) Represent the cell viability plot and $(\mathrm{b}, \mathrm{d}, \mathrm{f})$ represent the associated $\mathrm{IC}_{50}$ analysis. Results were averaged from 3 independent experiments. ${ }^{\star} \mathrm{p} \leq 0.05,{ }^{\star \star} \mathrm{p} \leq 0.01,{ }^{\star \star \star} \mathrm{p} \leq 0.001$, and the values were analyzed using one-way anova.

Figure 133. $72 \mathrm{~h}$ Toxicity profile for nanoparticles in H820, and HCC827 NSCLC cells. (a) Synthesized gelatin quenched and non-quenched NP and its representative (b) $\mathrm{IC}_{50}$ analysis, (c) Comparison between drug encapsulated nanoparticles, and its representative (d) $\mathrm{IC}_{50}$ analysis. Results were averaged from 3 independent experiments. 222 
Figure 134. Toxicity profile for siRNA conjugated nanoparticles in (a) $24 \mathrm{~h}$ data in H820, (b) $24 \mathrm{~h}$ data in HCC827 and (c) $72 \mathrm{~h}$ data in H820 cells. Results were averaged from 3 independent experiments. Based on the viability assay, $0.6 \mathrm{mg} / \mathrm{ml}$ was used as a treatment concentration for a period of $4 \mathrm{~h}$ in most MTT assays.

Figure 135. $72 \mathrm{~h}$ toxicity profile for synergistic drug and (a) siRNA conjugated nanoparticles and (a) erlotinib encapsulated siRNA constructs in H820 cells. Results were averaged from 3 independent experiments. ${ }^{\star}$ indicate $\mathrm{p} \leq 0.05,{ }^{\star \star}$ indicate $\mathrm{p} \leq 0.01$, and the values were analyzed using one-way anova. .224

Figure 136. Fluorescence imaging of mice treated with constructs and drug. 225

Figure 137. $(a, c)$ Total fluorescence measured from tumors and $(b, d)$ mean fluorescence measured from tumors. IT/IP/IV+ER represent dual-siRNA construct treatments. Results were averaged from 5 mice. ${ }^{\star} \mathrm{p} \leq 0.05$, and the normalized percentage differences between day 17 and day 20 values were analyzed using one-way anova. 226

Figure 138. (a) Protein blot for AXL and FN14 expression in mice tumor samples and (b) band densitometry analysis for the blot by normalizing values to PBS controls. Results for PBS, GAb+ER and GAbsi(AXL,FN14)+ER were averaged from 2, 5 and 5 mice tumors respectively. Results show AXL and FN14 expression is drastically reduced in siRNA conjugated gelatin nanoparticle treatment samples. .227

Figure 139. Resensitization to erlotinib in CRISPR assited AXL kncockout H820AK cell line for 72 h. (a) Represents the cell viability plot and (b) represent the associated IC $_{50}$ analysis. Results were averaged from 3 independent experiments. ${ }^{\star}$ indicate $\mathrm{p} \leq 0.05,{ }^{\star \star} \mathrm{p} \leq 0.01$, and the values were analyzed using one-way anova.. 228 


\section{List of Schemes}

Scheme 1. Schematic Illustration of the Synthesis of Bombesin Conjugated Gold Nanocages...........41

Scheme 2. Isolation of live CTC's using FeNCs assisted by magnetic capture. ................................78 


\section{List of Tables}

Table 1. Cells were exposed to NC6 after specific endocytic pathways were blocked using inhibitors. Visible gold signals from 100 individual cells were collected for analysis. Only representative images are provided here. (LIVE) Cells exposed to NC6 without pre-treatment of inhibitors were used as control. (DYN-BLOCK) Dynamin pathway blocked using dynasore, (CAV-M $\beta C D)$ caveolae pathway blocking using methyl- $\beta$-cyclodextrin, (CAV-FIL) caveolae pathway blocking using filipin, (CLTCLOR) clathrin pathway blocking using chlorpromazine, and (CLT-PIT2) clathrin pathway blocking using pitstop-2.

Table 2. Quantification of AuNC-BBN within PC3 cells using optical microscopy (dark field fluorescence illumination). NC6 was quantified as nanoaggregates within cells. (UNT) Cells exposed to NC6 without pre-treatment of inhibitors; (DYN) dynamin pathway blocked using dynasore; (CAV$\mathrm{M} \beta \mathrm{CD}$ ) caveolae pathway blocking using methyl- $\beta$-cyclodextrin; (CAV-FIL) caveolae pathway blocking using filipin; (CLT-X22AB) clathrin pathway inhibition using anti-clathrin antibody colocalization (CLT-CLOR) clathrin pathway blocking using chlorpromazine; and (CLT-PIT2) clathrin pathway blocking using pitstop-2. Results show decrease in AuNC-BBN uptake when CME was blocked.

Table 3. Statistical Analysis. (a) Wilcoxon Scores (Rank Sums) for Variable MICROSCOPY Classified by Variable inhibitors; (b) Kruskal-Wallis test; (c) Pairwise Two-Sided Multiple Comparison Analysis Dwass, Steel, Critchlow-Fligner Method; Variable: MICROSCOPY; (d) Wilcoxon Scores (Rank Sums) for Variable MICROSCOPY Classified by Variable Inhibitors; and (e) Wilcoxon Two-Sample Test. 39

Table 4. Physicochemical Properties of Gold Nanoconjugates 42

Table 5. Properties of FeNC constructs synthesized for the study .74

Table 6. Transmittance spectroscopy: Parameters of the 3 channels. 113

Table 7. Physicochemical properties of gelatin NP constructs. 185 


\section{List of Equations}

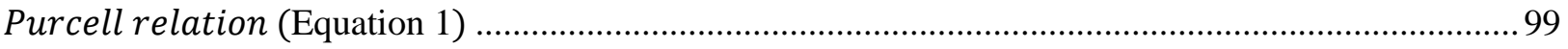

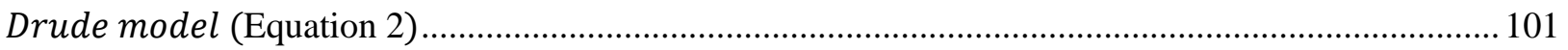

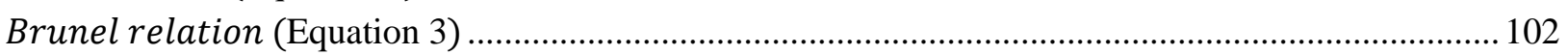

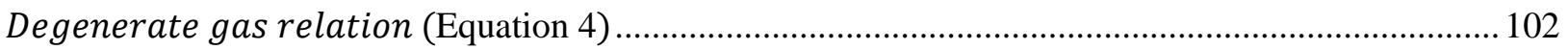

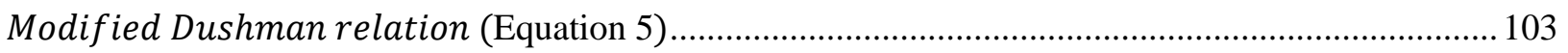

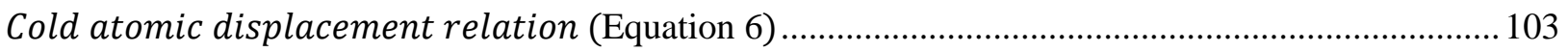

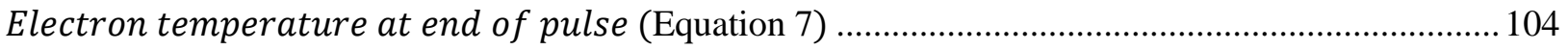

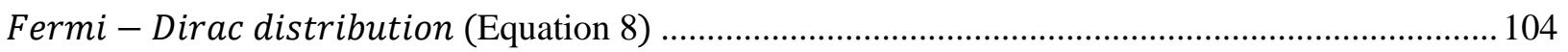

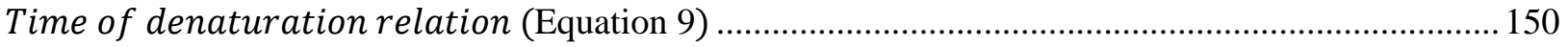




\section{List of Abbreviations}

4EBP1: Eukaryotic translation initiation factor 4E-binding protein 1

ADAM: A disintegrin and metalloproteinase

AFM: Atomic force microscopy

AgNC: Silver nanocubes

ALK: Anaplastic lymphoma kinase

AMPK: AMP-activated protein kinase

ANOVA: Analysis of variance

ANTH: AP180 N-terminal homology

AP180: Adaptor protein 180

AP2: Adaptor protein 2

ATP: Adenosine triphosphate

AuNC: Gold nanocage

AuNC: Gold nanocages

BAR: Bin/Amphimysin/Rvs domain

BBN: Bombesin

Cas9: CRISPR associated protein 9

CCV: Clathrin coated vesicle

CDK: Cyclin dependent kinase

CE: Computational electrodynamics

CG: Nanocages

CI-CI: Cancer immunotherapy checkpoint inhibitors

CME: Clathrin mediated endocytosis

CRISPR: Clustered regularly interspaced short palindromic repeats

CTC: Circulating tumor cells

CTLA4: Cytotoxic T-lymphocyte-associated protein 4

DAB-2: Disabled 2

DNA: Deoxyribonucleic acid

EDX: Energy-dispersive X-ray spectroscopy 
EELS: Electron energy loss spectroscopy

EGFR: Epidermal growth factor receptor

ELISA: Enzyme-linked immunosorbent assay

EMF: Electromagnetic fields

EMT: Epithelial to mesenchymal transition

ENTH: Epsin N-terminal homology

EpCAM: Epithelial cell adhesion molecule

EPR: Enhanced permeability and retention effect

EPS15: EGFR pathway substrate clone 15

ERK: Extracellular signal-regulated kinases

ESCRT: Endosomal sorting complex required for transport

EUVL: Extreme ultraviolet lithography

FAAS: Flame atomic absorption spectroscopy

FCHO: Fes/CIP4 homology domain only

FDA: Food and Drug Administration

FDTD: Finite-difference time-domain

FeNC: Iron nanocube

FISH: Fluorescence in situ hybridization

FOX: Forkhead box

FS LASER: Femtosecond light amplification by stimulated emission of radiation

GAK: Cyclin G-associated kinase

GAS-6: Growth-arrest-specific gene 6

GPCR: G protein coupled receptor

GRPR: Gastrin release peptide receptor

HAADF: High-angle annular dark-field imaging

HER: Human epidermal growth factor receptor

HGFR: hepatocyte growth factor receptor

HRS: Hepatocyte growth factor-regulated tyrosine kinase substrate

HSC70: Heat shock cognate protein 70

IHC: Immunohistochemistry 
IPA: Ingenuity Pathway Analysis

JAK: Janus kinase

JNK: c-Jun N-terminal kinases

KEGG: Kyoto Encyclopedia of Genes and Genomes

LSPR: Localized surface plasmon resonance

MAPK: Mitogen-activated protein kinase

MNP: Magnetic nanoparticle

mTOR: Mechanistic target of rapamycin

MZF1: Myeloid zinc finger 1

NAA: Neutron activation analysis

NC: Nanocubes

NEMS: Nanoelectromechanical systems

NF: Nanoframes

NFkB: Nuclear factor kappa-light-chain-enhancer of activated B cells

NGL: Next-generation lithography

NIM: Negative-index metamaterial

NP: Nanoparticle

NSCLC: Non-Small Cell Lung Cancer

N-WASP: Neural Wiskott-Aldrich syndrome protein

p70S6K: Ribosomal protein S6 kinase beta-1

PARP: Poly (ADP-ribose) polymerase

PD-L1: Programmed death-ligand 1

PI3K: Phosphatidylinositol-4,5-bisphosphate 3-kinase

PTEN: Phosphatase and tensin homolog

PTGS: Post-transcriptional gene silencing

RAB: Ras-related proteins in brain

RISC: RNA-induced silencing complex

RNA: Ribonucleic acid

RT-PCR: Reverse transcription polymerase chain reaction

SERF: Spin exchange relaxation-free 
siRNA: Short interfering RNA/ silencing RNA

SJ-1: Synaptojanin 1

SMAD: S-mothers against decapentaplegic

SNARE: Soluble N-Ethylmaleimide-sensitive factor attachment protein receptor

SPASER: Surface Plasmon Amplification by Stimulated Emission of Radiation

SQUID: Superconducting quantum interference device

STAT: Signal Transducer and Activator of Transcription

STEM: Scanning transmission electron microscopy

TEM: Transmission electron microscopy

TFSF: Total-field scattered-field source

TGF: Transforming growth factor

TKI: Tyrosine kinase inhibitor

TNFRSF12A: Tumor necrosis factor receptor superfamily member 12A

TP53: Tumor protein $\mathrm{p} 53$

tracrRNA: trans-activating crRNA

TUNEL: Terminal deoxynucleotidyl transferase dUTP nick end labeling 


\title{
How targeted nanoparticles enter cancer cells?
}

> Unraveling the mechanism of endocytosis using peptide conjugated nanocages

\begin{abstract}
Recently, nanomedicine development has largely focused on cancer cell targeted drug delivery methods. Primarily nanoparticles are being used to target overexpressed receptors on cancer cells using peptide biomarkers. However, the nature of interaction and mechanism of internalization of receptor-avid peptide nanoparticles with cells is not yet completely understood, leading to noticeable therapeutic inefficacy. The therapeutic efficiencies of nanoparticle delivery systems depend on two important parameters: (i) the ability to selectively recognize and internalize within a cancer cell and (ii) the ability to release a payload from endosomes to the cancer cell cytoplasm. Thus if nanoparticles can be targeted to specific pathways they can achieve the desired therapeutic effect. Therefore, it is extremely important to understand the internalization mechanism. Cancer cells use multiple biochemical pathways to internalize molecules and these pathways are sometimes vital for their oncogenic imbalance and continued survival. The predominant pathways are clathrin, caveolae, macropinocytosis and phagocytic pathways. Recent research has shed light on adaptor proteins utilized in these pathways and therefore it is possible to manipulate these systems for our benefit. In order to investigate intracellular trafficking, endocytosis of peptide conjugated receptor targeted porous gold nanocages (AuNCs) was studied in prostate cancer cells (PC3). The porosity of the particle enhances peptide loading while prostate cancer was chosen as the model disease as it accounted for nearly $29 \%$ (i.e., 241740 estimated cases) of the total male cancer cases in the year 2012 alone.
\end{abstract}




\section{METHODS}

For this purpose, synthesis and characterization of a library of AuNCs conjugated with bombesin $(\mathrm{BBN})$ peptide was carried out. Bombesin peptide is known to target overexpressed Gastrin releasing peptide receptors (GRPR) on the surface of PC3 cells. Peptide was conjugated using gold-thiol chemistry, characterized using UV-Visible absorption spectroscopy and quantified using high performance liquid chromatography (HPLC). Evidence of selective affinity of AuNC-BBN toward gastrin releasing peptide receptors (GRPR) was obtained using radiolabeled competitive cell binding assay and fluorescence dark field microscopy. Cytotoxicity of the nanoconjugates were determined by MTT assay and endocytic mechanism was investigated using cell inhibitor studies monitored through optical and transmission electron microscopy (TEM). Finally, cells were isolated using flow cytometry and the internalized particles were quantified using neutron activated analysis (NAA).

\section{RESULTS}

Results show AuNC-BBN uptake in PC3 cells is mediated by clathrin mediated endocytosis (CME). In the presence of CME inhibitors, AuNC-BBN uptake in cells is reduced up to $84 \%$. TEM images further confirm CME characteristic clathrin coated pits and lysosomal release of AuNCs within cytoplasm. These results demonstrate that peptide ligands conjugated to the surface of nanoparticles maintain their target specificity and validates the mechanism through which nanoparticles interact with the cell plasma membrane through receptors on the surface. Subsequently this study establishes how cubic porous nanocages can be tailored for cytoplasmic delivery necessary for efficient drug delivery. 


\section{DISCLOSURE:}

This work has been published in Bioconjugate Chemistry, Volume 25, 8, Pages 1565-1579 in 2014 under the title "Bombesin Peptide Conjugated Gold Nanocages Internalize via Clathrin Mediated Endocytosis."

It is reprinted with permission (C American Chemical Society 2014). Dhananjay Suresh, Ajit

Zambre, Nripen Chanda, Timothy J. Hoffman, C. Jeffrey Smith, J. David Robertson, and Raghuraman Kannan are co-authors of this work. 


\section{CHAPTER 1}

\subsection{INTRODUCTION}

Traditionally, chemotherapeutic agents or drugs are directly injected or administered orally in patients. These drugs have a tremendous effect on cancer cells due to their sensitivity, but their non-selective action can affect nearby tissues as well. To decrease this toxicity, increasing the efficacy of the drug plays an important role. As a solution to this problem, numerous studies have shown nanoparticles can aid in drug delivery to the tumor with high efficacy $^{1-3}$. This increase in efficacy is because of the high drug loading capacity of the nanoparticles. However, these nanoparticles can also accumulate in various tissues imparting a high drug dose and increasing side effects ${ }^{4,5}$. For this reason, a new field of targeted drug delivery is being developed in current oncology. Although, attempts to achieve an efficient targeted nanoparticle are being made, the mechanism of action is not yet fully understood ${ }^{6}$. This hampers the nanoparticle design process. Therefore, in this study we focus on designing a drug delivery agent that can be targeted to cancer cells and unravel the mechanism of nanoparticle action in cancer cells.

\subsubsection{Current cancer therapy}

Currently, liposomal and albumin complexes are being used for drug delivery ${ }^{7}$. But these particles are not targeted and they still lead to non-selection action in noncancerous tissues. This in turn increase dosage requirements and duration of treatment. These decisions often worsen the quality of life in patients and sometimes leads to acquired drug resistance ${ }^{8}$. In order to reduce these side effects and dosage requirements we need to design a nanoparticle that not only acts as a drug delivery vehicle to transport 
cytotoxic drugs but has (i) the ability to selectively target cancer cells and (ii) and release in the cancer cell cytoplasm rather than lysosomes for effective drug action ${ }^{9}$. The targeting abilities and eventual cytoplasmic release are influenced by molecular processes known as endocytosis. Based on the nature of endocytosis nanoparticles can enter cells, target intracellular areas or exit cells. By tailoring the factors such as shape, size and type of biomolecules on the nanoparticle surface, we can modulate cellular interaction and cytoplasmic release ${ }^{10,11}$. Cellular contact often involve interaction with the plasma membrane of cancer cells. In order to target cells these nanoparticles would have to target biomolecules on the surface such as receptors that are specifically present on cancer cells. However, a unified biomolecular mechanism for cellular uptake and fate of nanoparticles through receptor targeting in cells has not been fully understood. Therefore, a study focused on understanding the endocytic mechanism of targeted nanoparticles is vital for the design and development of therapeutically efficient nanoparticle-based delivery systems.

\subsubsection{Receptor mediated endocytosis}

Recent studies have shown that functional biomolecules such as peptides or antibodies can be used to target receptors based on their biomolecular affinity. These biomolecules can be attached on the surface of nanoparticles to target and enter cancer cells via endocytic pathways. There are several endocytic pathways in cells such as clathrin, caveolae, macropinocytosis or phagocytosis, out of which clathrin mediation is commonly used for receptor internalization (Figure 1) ${ }^{12}$. This means that if the targeted nanoparticles use the 


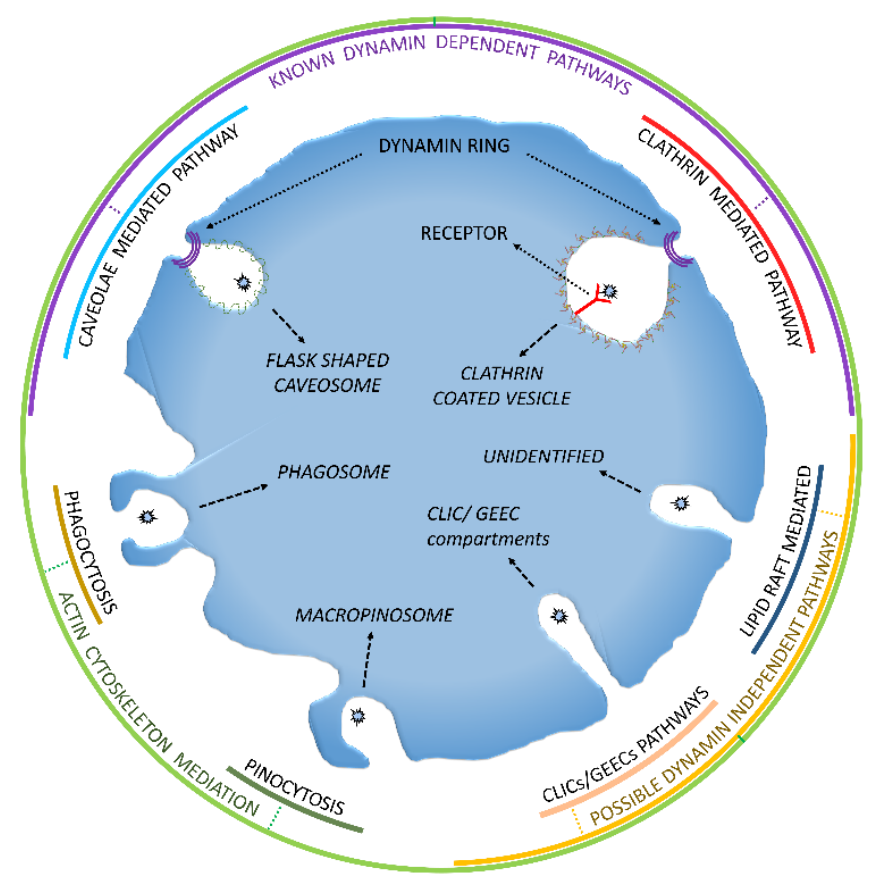

Figure 1. Cell Pathways involved in endocytosis.

clathrin mediated mechanism it would signify receptor targeted uptake in cells. The clathrin mediated pathway involve a host of biomolecules adaptor, recruiter and framework proteins such as dynamin, AP2 and clathrin. Clathrin molecules are responsible for the formation of a characteristic network to form stable pits at the membrane interface. These clathrin coated pits are internalized by vesicular budding and scission initiated by dynamin, to form small vesicles often with a size limit of 10$300 \mathrm{~nm}^{13}$. These vesicles follow a series of early and late endosomal stages followed with their merging with lysosomes for eventual degradation within the cells ${ }^{14}$. Lysosomes at the final stage are used for cellular degradation processes and have an acidic $\mathrm{pH}^{15}$. Therefore, it is essential to consider a pre lysosomal release for a more effective drug delivery in cells to avoid lysosomal degradation. Considering these factors, the nanoparticle has to be designed to either escape the endosomal or lysosomal vesicles by rupturing or by bursting. 


\subsubsection{Drug delivery vehicle}

In order to design a perfect drug carrier, a nanoparticle needs to have specific requirements. These requirements include (1) biological inertness, (2) surface reactivity for biomolecule functionalization, (3) porosity for drug loading and shielding, and (4) ability to rupture vesicles. In order to have biological inertness, the material used should be cytocompatible at high concentrations. Recent studies have shown noble metals such as gold do not impart toxicity even after accumulating in cells at high levels ${ }^{16}$. Therefore, gold can be an ideal material for synthesizing targeted nanoparticles. Furthermore, gold has affinity to thiols that make them easy for surface functionalization. Several studies have shown covalent conjugation of molecules involving sulfhydryl groups to gold surface ${ }^{17,18}$. Gold nanoparticles can also be made porous by using etching reactions to maximize available volume within the particle to house drugs ${ }^{18,19}$. These type of particles known as gold nanocages (AuNCs) have high payload capacity and have been shown to achieve controlled delivery of drugs to desired cellular sites ${ }^{20}$. Controlled delivery can be achieved by activating these particles using lasers due to their strong absorption in the near infrared region. AuNCs also have a cubic shape that has been shown to be more superior in rupturing from vesicles than other shapes such as spheres or rods ${ }^{21,22}$. Additionally, the cubic shape also imparts intense light scattering abilities that are easy to detect and image. Considering these features, we focused on the use of AuNCs for targeting cancer cells. In order to target, we chose the gastrin releasing peptide receptor (GRPR) as a candidate as they are overexpressed in many human cancers, such as prostate, breast, and lung cancers ${ }^{23,24}$. Among these cancers, GRPR is especially seen to be upregulated in prostate cancer. 
Based on American cancer society statistics for total male cancer cases in the US, prostate cancer accounts for nearly 1,658,370 new cancer cases and 589,430 cancer deaths in the year 2015 alone. Similarly, a study involving 36 human prostate tumor patients revealed that a large majority $(83 \%)$ of the patients showed very high density of GRPR (>1000 dpm/mg tissue) in tumor tissue ${ }^{25}$. Based on this information, we decided to target the GRPR using AuNCs.

\subsubsection{Molecular agents for targeting prostate cancer}

In order for the nanoparticle to target GRPR, the nanoparticle has to be functionalized with a biomolecule that has affinity towards that receptor. If the biomolecule attaches to the receptor it would initiate a clathrin mediated endocytosis. For example, gold nanoparticles (AuNPs) conjugated to transferrin endocytosed via a clathrin-mediated endocytosis pathway in a previous $\operatorname{study}^{26}$. However, AuNPs conjugated with Cetuximab (C225) antibody were observed to use the caveolae-mediated mechanism ${ }^{27}$. Based upon these experiments, it is evident that the mechanism of endocytosis depends not only on physicochemical properties, but on specific ligands conjugated to the nanoparticle surface. The endocytic mechanisms for nanoparticles proposed so far have been regarding an antibody or small organic molecule as the targeting agent ${ }^{26,28-31}$. However, large molecules such as an antibody, can decrease loading efficiency due to steric hindrance. Similarly, small molecules may not be suitable for many applications. In this regard, peptides can be a replacement because of their low immunogenic properties, established synthetic chemistry and low steric hindrance ${ }^{32,33}$. But mechanism for intracellular trafficking of receptor-avid peptide 
conjugated AuNPs in human cancer cells are not fully known. Still, several studies have shown these conjugates have good clinical applications ${ }^{34-37}$. In one study, a 14-aminoacid Bombesin (BBN) peptide conjugated gold nanoparticles (AuNPs) were shown to have both in vitro and in vivo affinity for the GRPR ${ }^{34}$. Similarly, BBN was shown to target GRPR in vivo in a separate study ${ }^{38}$. Based upon these results, we hypothesized that BBN conjugated AuNC would target the GRPR on human prostate cancer cells and follow a receptor-mediated endocytosis (clathrin) mechanism. To confirm this hypothesis, this study focused on the mechanistic interactions between GRPR and AuNC-BBN conjugates in PC3 cells. A library of conjugates was tested for both affinity as well as to understand the effect of surface ligands on endocytic mechanism.

\subsection{METHODS AND CHARACTERIZATION}

\subsubsection{Materials}

Distilled water was used for all chemical experiments and synthesis. Hypure cell culture grade water from Thermo-Scientific was used for all cellular experiments. 0.1\% TFA, acetonitrile, silver nitrate, anhydrous ethylene glycol, sodium hydrosulfide, polyvinyl pyrrolidone (PVP), hydrochloroauric acid, propidium iodide, RPMI cell medium, TrypLE buffer, sodium chloride, bovine serum albumin, human serum albumin, cysteine, histidine, phosphate buffer saline, sodium cyanide, chlorpromazine hydrochloride, amiloride hydrochloride hydrate, methyl- $\beta$-cyclodextrin, cytochalasin D, dynasore hydrate, uranyl acetate, and poly-L-lysine solution were all purchased from Sigma-Aldrich. Prolong gold antifade reagent with DAPI, CellMask Deep Red plasma membrane stain, and ZebaTM Micro spin desalting column (7K MWCO) were 
purchased from Thermo-Scientific. X-22 mouse-monoclonal anti-clathtrin antibody and Pitstop-2 were purchased from Abcam. Custom methoxy-polyethyleneglycol-thiol (mPEG-SH; M.W 750) was purchased from Rapp Polymere GmBH. Custom modified peptide, Bombesin (Lipoic-QWAVGHLM-NH2) was designed in-lab and synthesized by CPC Scientific.

\subsubsection{Instrumentation}

Metal concentrations were estimated using Flame Atomic Absorption Spectroscopy (fAAS). Nanoconstructs were analyzed using a Jeol HR-TEM. $85 \mathrm{~nm}$ cell cross sections for TEM was performed using Leica ultracut UCT microtomes with a diamond knife. Cellular analysis was performed on the Cytoviva dark field fluorescence microscope system. Hyperspectral images for gold nanocages in cell samples were captured using a Cytoviva VNIR hyperspectral imaging system with a $500 \mathrm{~nm}$ long pass filter to eliminate harmonics (Spectral imager by Headwall Photonics; Mounted Dage-MT camera; Aluminum Bulb with $400 \mathrm{~nm}-950 \mathrm{~nm}$ range). Hyperspectral data was analyzed using modified ITT ENVI image processing software. Nexterion Glass B laser cut slides and Nexterion Glass B coverslips (ultrasonically cleaned) purchased from Schott were utilized for microscopy. Centrifugation was performed on a 5424 Eppendorf and refrigerated RC 6+ Sorvall centrifuge. Cell sorting was performed using a Beckman Coulter MoFlo XDP flow cytometer. UV measurements were performed using a Varian Cary Bio 50 UV-vis multi sampler spectrophotometer in quartz cuvettes. Retention times were calculated using a Schimadzu reverse-phase HPLC system (4.6x250mm) with a Phenomenex C18-5 $4300 \AA ̊$ column. HPLC columns utilized a two 
gradient solvent method: (a) $0.1 \%$ TFA in $100 \%$ acetonitrile and (b) $0.1 \%$ TFA in $100 \%$ water. For measuring core size, $8 \mu \mathrm{l}$ of nanoparticle suspension was air dried for $20 \mathrm{~min}$ over a CF300-Cu carbon film based copper grid. For measuring hydrodynamic size and zeta potential, a Malvern Zetasizer Nano-ZS was used. For calculating particle size distribution, a Nanosight (nanoparticle tracking analysis) was used. Absorbance and MTT assay measurements were analyzed using Nano drop and Gen5 2.0 Biotek Epoch (Take3/standard plate) reader system. Radiolabeled $\mathrm{IC}_{50}$ assays were analyzed using a Packard Riastar $\gamma$-counter. Irradiated ${ }^{198} \mathrm{Au}$ was estimated using a high purity germanium detector (HPGe) at the Research reactor. For automated liquid injection in synthetic procedures a KDS-200 series syringe pump was used.

\subsubsection{Synthesis of Nanocages (NC1)}

Nanocages were synthesized using galvanic reduction of silver nanocubes as previously reported by Xia and co-workers ${ }^{39}$. Nanocubes were synthesized by a slightly modified polyol reduction process explained as follows: $60 \mathrm{~mL}$ ethylene glycol was taken in a (3-necked) $250 \mathrm{~mL}$ round-bottom flask and heated to $150{ }^{\circ} \mathrm{C}$ under constant stirring around $350 \mathrm{rpm}$ for $50 \mathrm{~min}$. Argon gas at $20 \mathrm{kPa}$ was introduced through a nozzle above the solution for $10 \mathrm{~min}$. After initial argon flushing, the rest of the reaction remained under argon atmosphere (10 kPa). $0.7 \mathrm{~mL}$ of sodium hydrosulfide dissolved in ethylene glycol (3 mM) and $15 \mathrm{~mL}$ of polyvinylpyrrolidone (PVP) dissolved in ethylene glycol (20 mg/mL; MW 55 000) were then added in quick succession. After $8 \mathrm{~min}, 5 \mathrm{~mL}$ of $\mathrm{AgNO}_{3}$ dissolved in ethylene glycol $(48 \mathrm{mg} / \mathrm{mL})$ was quickly added into the reaction solution. Reaction turned from a light yellow solution (at $1 \mathrm{~min}$ ) to green ochre (at 20 
min). The reaction was stopped at this moment and cooled in an ice bath for $5 \mathrm{~min}$ followed with addition of $40 \mathrm{ml}$ of acetone. The reaction mixture was centrifuged and the pellet was washed 6 times with water to remove excess PVP. Silver nanocubes were then dissolved in $10 \mathrm{~mL}$ water and titrated with $\mathrm{HAuCl}_{4}(0.9 \mathrm{mM}$; injected at $45 \mathrm{ml} / \mathrm{h}$ using a syringe pump) in the presence of $10 \mathrm{ml}$ of PVP (2 mg/mL; MW 55000$)$ as a stabilizer in a (2-necked) $300 \mathrm{~mL}$ round-bottom flask. Reaction was performed at 100 ${ }^{\circ} \mathrm{C}$ under constant stirring (400 rpm) for 5 min. Reaction was stopped when the suspension gave a UV-vis absorbance maximum at $750 \mathrm{~nm}$ by cooling in an ice bath for $5 \mathrm{~min}$. The solution was centrifuged $(7000 \mathrm{~g})$ for $20 \mathrm{~min}$. Supernatant was discarded and the pellet was washed with water containing $400 \mu \mathrm{L}$ of $\mathrm{NH}_{4} \mathrm{OH}$ to remove $\mathrm{AgCl}$ from the product. Subsequently, the solution was centrifuged 6 times with water to remove excess PVP (10000g for $10 \mathrm{~min})$. Final purified product NC1 (50 mg yield) was further concentrated and freeze-dried for storage at $-20^{\circ} \mathrm{C}$.

\subsubsection{Synthesis of NC2 and NC3}

NC1 $(5 \mathrm{mg})$ was resuspended in $2 \mathrm{~mL}$ of water by sonicating for $10 \mathrm{~min}$ in a $5 \mathrm{ml}$ glass vial. To this solution, thiolated BBN (MW 1129) dissolved in $2 \mathrm{~mL}$ methanol was added according to 1:1/1:3 Gold-BBN molar ratio (unless otherwise indicated, all experiments utilized the 1:3 ratio) and the suspension was stirred at $200 \mathrm{rpm}$ for $12 \mathrm{~h}\left(23{ }^{\circ} \mathrm{C}\right)$. Reaction suspension was centrifuged at $10,000 \mathrm{~g}$ for $10 \mathrm{~min}$ to obtain a pellet. Pellet was washed twice with methanol to remove free bombesin, followed by 6 washings in water. Final products (AuNC-BBN-PVP) were labeled NC2 (1:1 ratio) and NC3 (1:3 ratio). Products were further concentrated, freeze-dried, and stored at $-20{ }^{\circ} \mathrm{C}$. 


\subsubsection{Synthesis of NC5 and NC6}

$\mathrm{NC} 1(5 \mathrm{mg})$ was resuspended in $2 \mathrm{~mL}$ of water by sonicating for $10 \mathrm{~min}$ in a $5 \mathrm{ml}$ glass vial. To this solution, thiolated PEG (MW 750) dissolved in $2 \mathrm{~mL}$ water was added (1:3 Gold-PEG molar ratio) and the suspension was stirred at $200 \mathrm{rpm}$ for $12 \mathrm{~h}\left(23{ }^{\circ} \mathrm{C}\right)$. Reaction mixture was centrifuged followed with 6 washes in water to obtain NC4. Subsequently, NC4 was incubated with modified thiolated Bombesin peptide (LipoicQWAVGHLM-NH2) to bind to gold surface. Thiolated BBN (MW 1129; 1:1/1:3 GoldBBN molar ratio (unless otherwise indicated all experiments utilized the 1:3 Au:BBN ratio) dissolved in methanol was added to $2 \mathrm{ml}$ of NC4 in a glass vial and the reaction was stirred at $200 \mathrm{RPM}$ for $12 \mathrm{~h}\left(23^{\circ} \mathrm{C}\right)$. Resulting suspension was centrifuged at $10000 \mathrm{~g}$ for $10 \mathrm{~min}$, and the pellet was washed twice with methanol to remove free $\mathrm{BBN}$, followed by 6 washings with water. Final products (AuNC-BBN-PEG) were labeled NC5 (1:1) and NC6 (1:3). Products were further concentrated, freeze-dried, and stored at $-20^{\circ} \mathrm{C}$.

\subsubsection{Characterization of Nanoconstructs}

Nanoconstruct properties were characterized using UV-vis spectroscopy, TEM, DLS, and NTA. Silver nanocubes had a UV-vis absorption at $410 \mathrm{~nm}$ shown in Figure 2. The silver nanocubes are etched with gold salt resulting in formation of AuNCs with increase porosity that have a broad UV-vis absorption at $700 \mathrm{~nm}$ (Figure 3). TEM images confirm the formation of hollow cubic shape of nanocages from silver nanocubes as shown in Figure 4 and Figure 5 and the thickness of the outer shell was estimated to be $\sim 7.0 \mathrm{~nm}$. We also recorded the hydrodynamic size and $\zeta$-potentials. 
Resulting AuNCs had a uniform size distribution as confirmed by nanoparticle tracking analysis (NTA) and a cubic particle yield of 70\% based on TEM analysis (Figure 6).

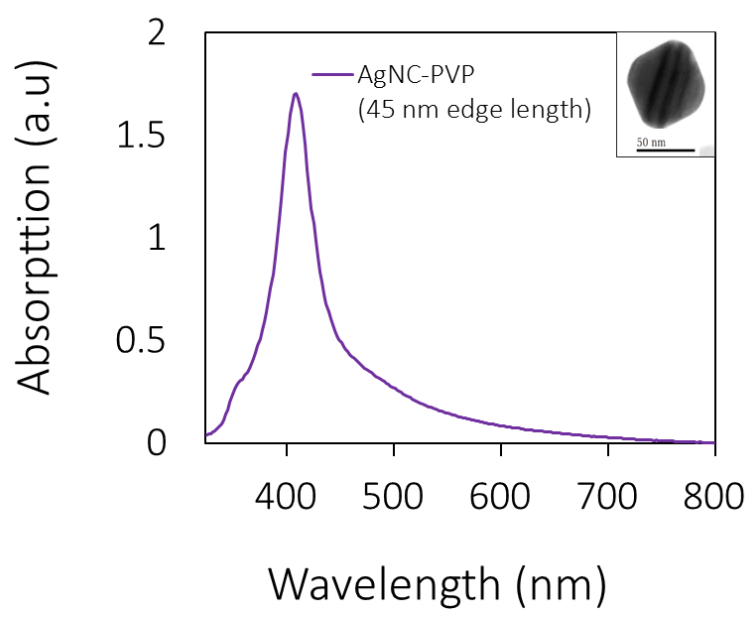

Figure 2. UV-vis absorption spectrum for silver nanocubes

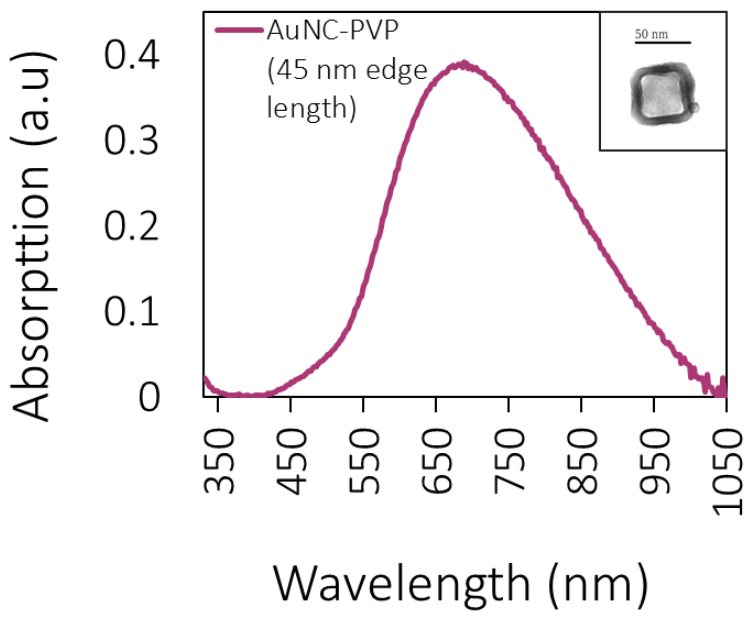

Figure 3. UV-vis absorption spectrum for gold nanocages 
a

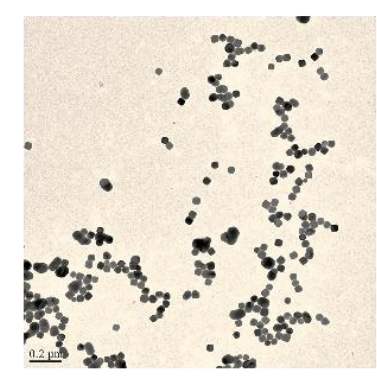

C

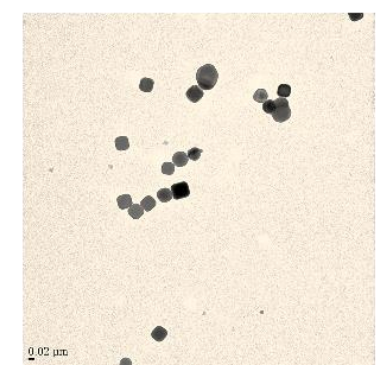

b

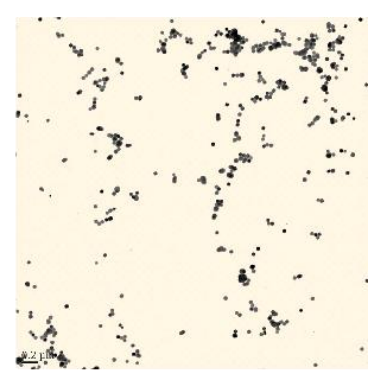

d

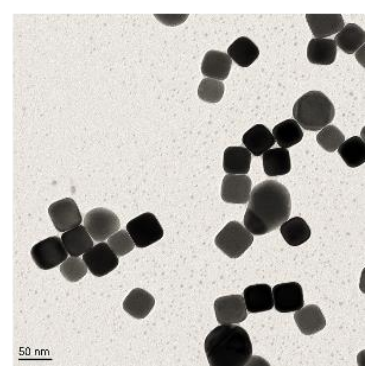

Figure 4. (a-d) TEM images for silver nanocubes

a

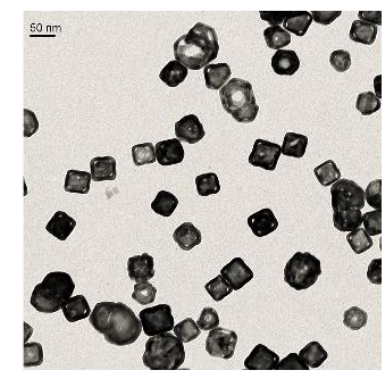

C

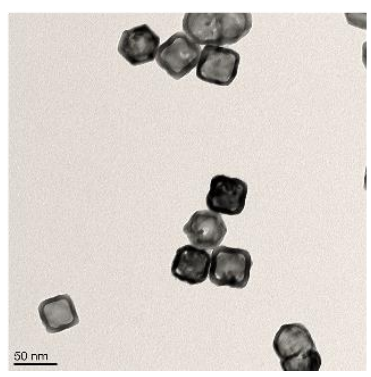

b

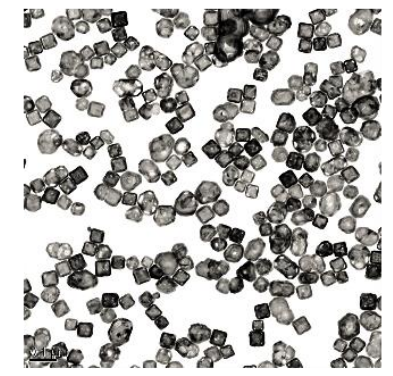

d

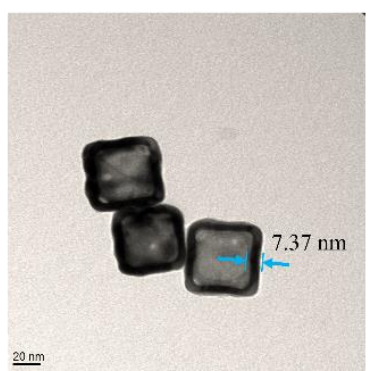

Figure 5. (a-d) TEM images for gold nanocages 
a
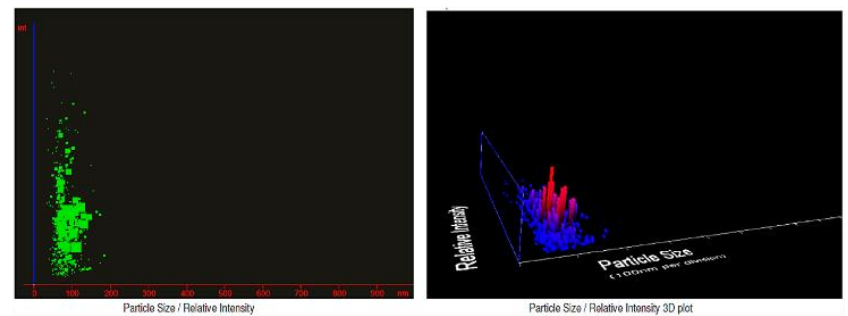

RESULTS:

Size Distribution:

Cumulative Data:

User Lines:

Total Concentration:

Selected Concentration:

Fitted Curve 1:

Fitted Curve 2:

Dritt Velocity:
Mean: 90nm Mode: $73 \mathrm{~nm}$ SD: 23nm D10: 64nm, D50: $86 \mathrm{~nm}$, D90: 123nm, D70: $99 \mathrm{~nm}$

Onm, Onm

20.17 particles / frame, $2.68 E 8$ particles $/ \mathrm{ml}$

Mode: 0.00 prm, SD: 0, Fitted Share: $0.0 \%$

Mode: $: \mathrm{nm}, \mathrm{SD}: 0$, Fitted Share: $0.0 \%$
Mode: $0 \mathrm{~nm}, \mathrm{SD}: 0$, Fitted Share: $0.0 \%$

480

$1747 \mathrm{~nm} / \mathrm{s}$ b

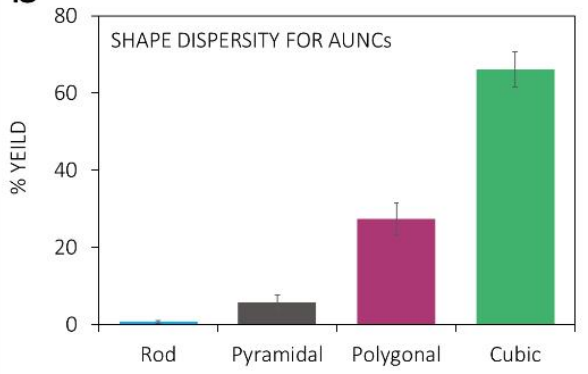

Figure 6. (a) Size distribution indicates AuNCs synthesized are of uniform size (b) Shape dispersity plot for AuNCs synthesized

\subsubsection{Peptide estimation}

The amount of bombesin conjugated to nanoparticles was estimated by dissolving nanoparticles in methanol followed by gold leaching using sodium cyanide. As gold dissolves, bombesin from the surface is released as free peptide. Subsequently, amount of free peptide was measured using its UV-vis absorption at $280 \mathrm{~nm}$. Each nanoconstruct with similar amounts were separately added in two glass vials. Pure methanol was used as a solvent for the control vial while $\mathrm{NaCN}$ dissolved methanol was used for the digestion vial. After 5 min of digestion, the solution turned transparent indicating all the gold was dissolved. The solutions were then subjected to UV-vis measurements and results were compared with controls. Measurements were performed for both $230-380 \mathrm{~nm}$ and $220-950 \mathrm{~nm}$ to monitor BBN peak and digestion of AuNCs respectively. In case of NC3 and NC6, the digested solutions were further centrifuged two times in order to obtain a supernatant, free of any remaining precipitates. The absorption of peptide was recorded by both UV spectroscopy and HPLC, and amounts 
were calculated by using a Bombesin standard concentration curve. Bombesin peptide (SS-BBN; $0.4 \mathrm{mg} / \mathrm{mL}$ ) was also dissolved in $\mathrm{NaCN}$ as a control showing no change in absorption properties.
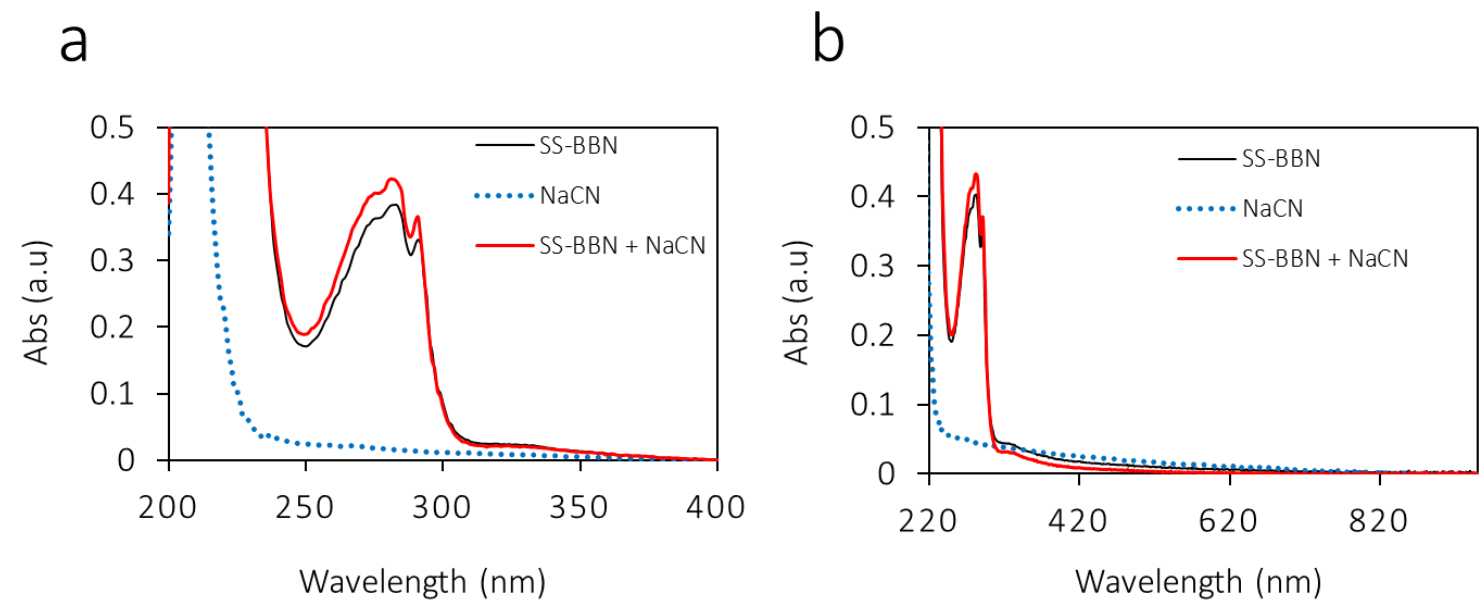

Figure 7. UV-visible spectroscopy studies using SS-BBN peptide. SS-BBN peptide was treated with NaCN and spectra was recorded at different wavelengths to ensure the structural integrity of the peptide. (a) SS-BBN peak at $280 \mathrm{~nm}$ does not shift in presence of $\mathrm{NaCN}$ solution. (b) No absorption in the NIR region
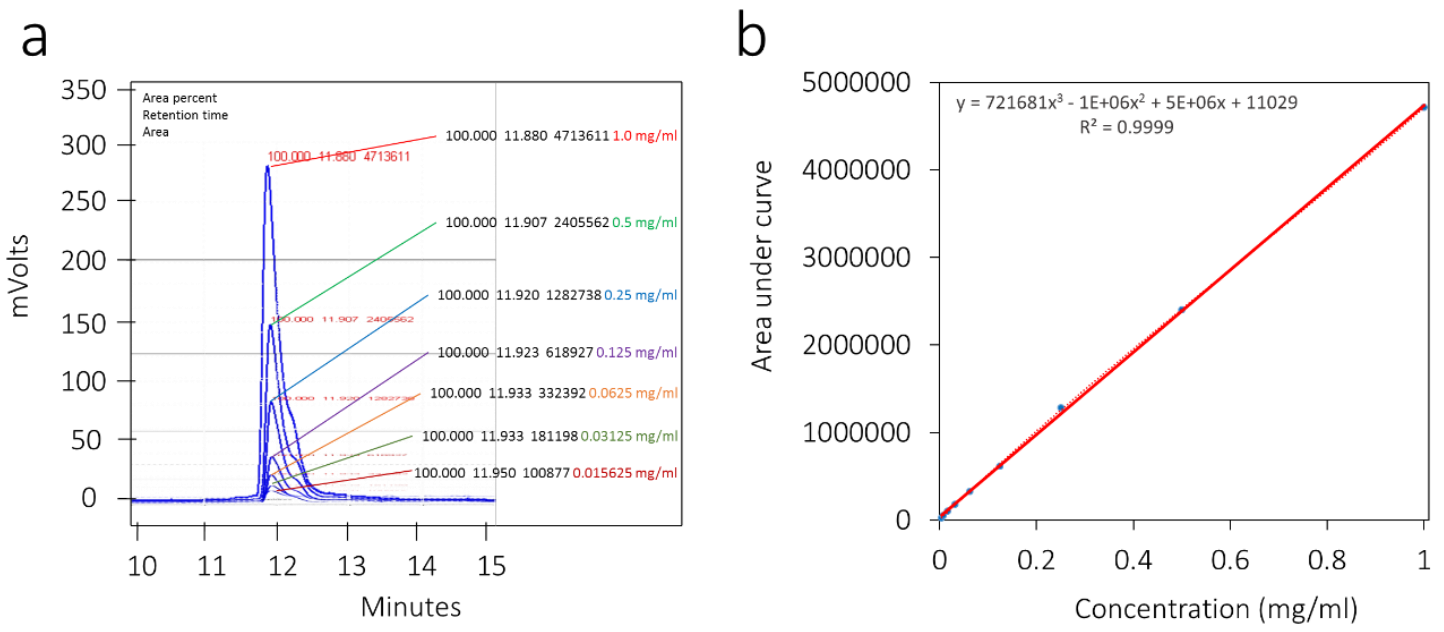

Figure 8. Estimation of bombesin using HPLC. (a) Retention curves of SS-BBN peptide in different concentrations; (b) 3rd order polynomial standard curve constructed using area under curves of SS-BBN at different concentration levels. (Detector wavelength: $280 \mathrm{~nm}$; Column flow rate $1.0 \mathrm{ml} / \mathrm{min}$ with $20 \mu \mathrm{l}$ volume of injection) 
a

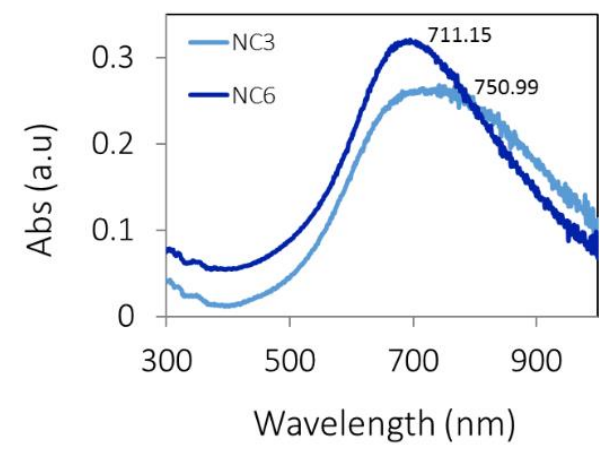

b

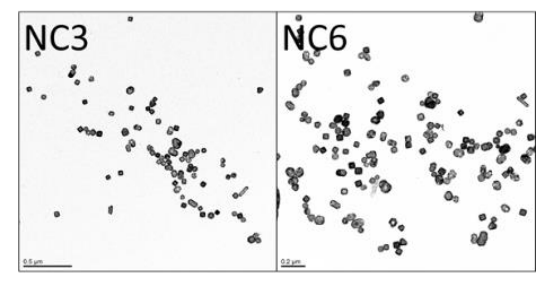

Figure 9. (a) UV-Visible spectra and (b) TEM images of NC3 and NC6. NC3 and NC6 are bombesin conjugated gold nanocages. Plasmon absorption of both nanoconstructs at NIR region indicates the size and shape similarity.
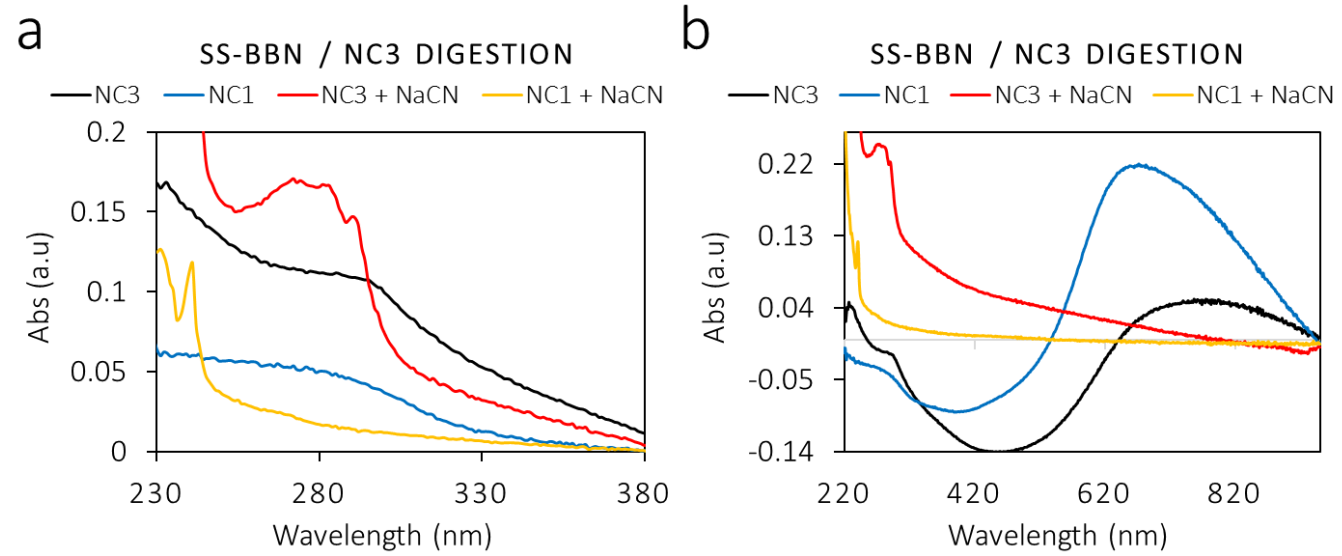

Figure 10. UV-visible spectra for NC3 or NC1 at different wavelength ranges before and after NaCN digestion. (a) NC3 sample showed absorption band at $280 \mathrm{~nm}$ corresponding to BBN peptide, whereas no corresponding peak was observed for NC1. (b) After digestion, plasmon absorption band for AuNC disappears

SS-Bombesin showed a peak at around $280 \mathrm{~nm}$ (Figure 7) as confirmed by UV-Vis spectroscopy. Similarly, SS-BBN monitored by a UV detector (280 nm) showed a retention time of $11.9 \mathrm{~min}\left(\mathrm{CH}_{3} \mathrm{CN}-\mathrm{H}_{2} \mathrm{O}\right.$ gradient system) in HPLC analysis (Figure 8). Control UV-vis spectra and TEM images of NC3 and NC6 are shown in Figure 9. After $\mathrm{NaCN}$ digestion, SS-BBN in the supernatant was observed for NC3, while for its control NC1 (devoid of SS-BBN on surface) no corresponding absorption peak was 
observed (Figure 10 and 12). Similarly, the peak for SS-BBN was visible after NC6 digestion, while for its control NC4 (devoid of SS-BBN on surface) no corresponding peak was observed after nanocage digestion (Figure 11 and 12). Standard curves for estimating bombesin are shown in Figure 8 and 13.

a

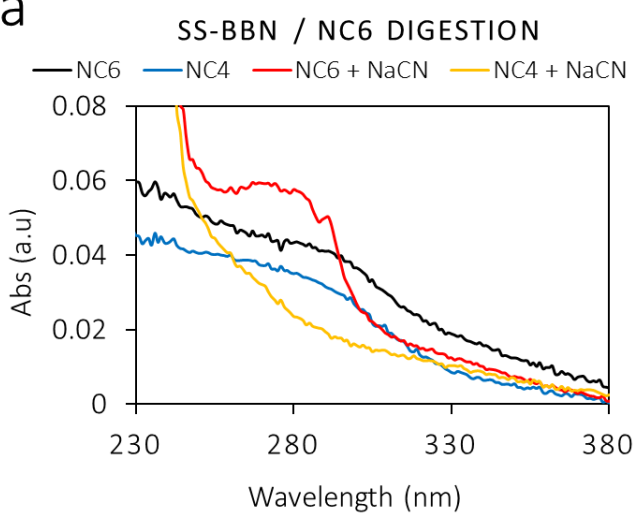

b

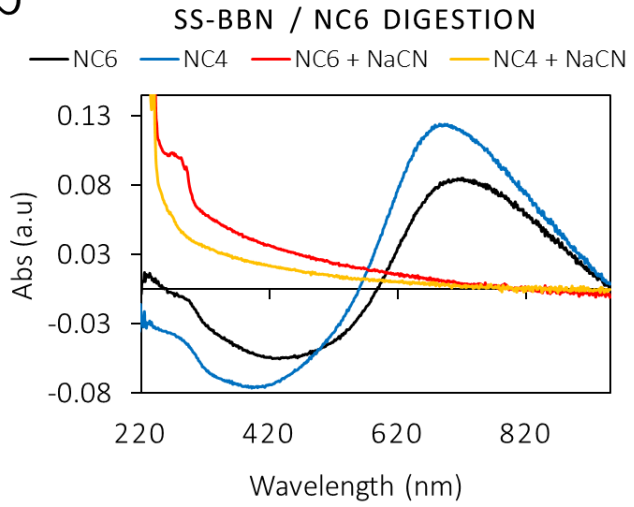

Figure 11. UV-Visible spectra for NC6 or NC4 at different wavelength ranges before and after NaCN digestion. (a) NC6 sample showed absorption band at $280 \mathrm{~nm}$ corresponding to BBN peptide, whereas no corresponding peak was observed for NC4. (b) After digestion, plasmon absorption band for AuNC disappears

a

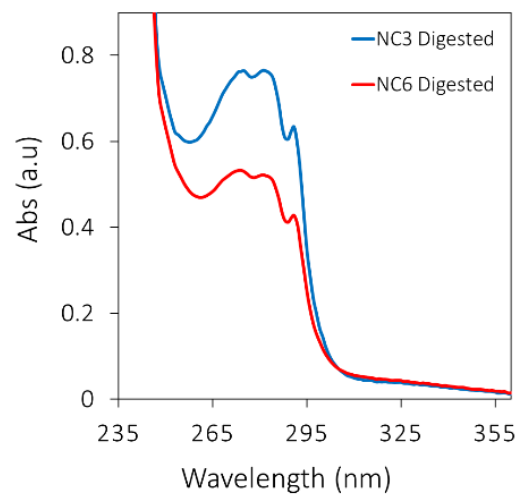

b

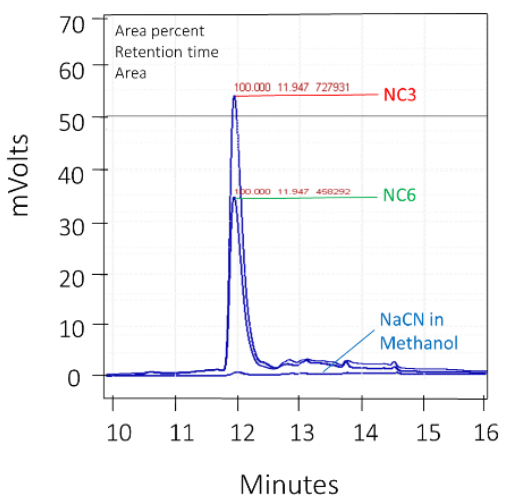

Figure 12. Estimation of bombesin using UV-visible spectroscopy and HPLC. (a) UV-visible spectra for digested and centrifuged NC3 and NC6 samples (dry powder); (b) HPLC retention curves for digested and centrifuged NC3 and NC6 samples (dry powder). Results from UV-visible spectroscopy corroborate with that of HPLC data. (HPLC: Detector wavelength: $280 \mathrm{~nm}$; Column flow rate $1.0 \mathrm{ml} / \mathrm{min}$ with $20 \mu \mathrm{l}$ volume of injection) 

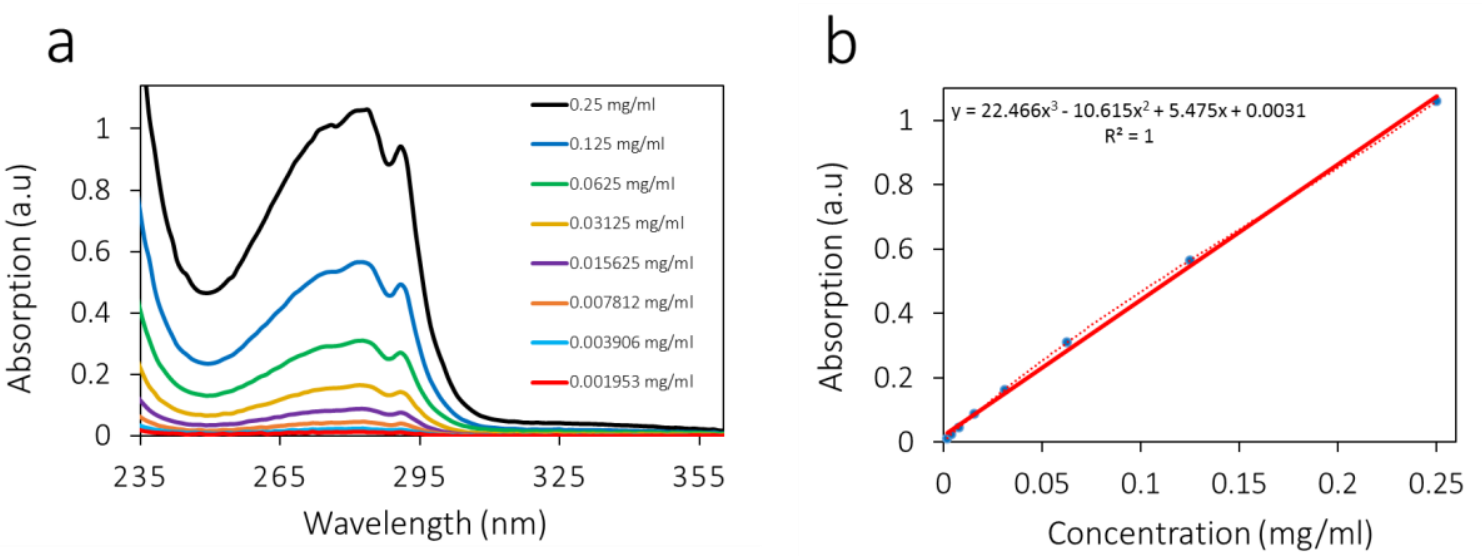

Figure 13. Estimation of bombesin using UV-visible spectroscopy. (a) UV-visible spectra of SS-BBN peptide in different concentrations; (b) 3rd order polynomial standard curve constructed using absorption of SS -BBN at different concentration levels.

\subsubsection{Cell Culture}

PC3 human prostate carcinoma cells (ATCC, USA) were grown in RPMI 1640 medium (obtained from Gibco BRL). The media was supplemented with $4.5 \mathrm{~g} / \mathrm{L} \mathrm{d}$-glucose, 25 $\mathrm{mM}$ Hepes, $0.11 \mathrm{~g} / \mathrm{L}$ sodium pyruvate, $1.5 \mathrm{~g} / \mathrm{L}$ sodium bicarbonate, $2 \mathrm{mM}$ 1-glutamine, $10 \%$ heat-inactivated fetal bovine serum (Atlanta Biologicals, USA), and 1\% penicillin/streptomycin antibiotic solution. Cells were cultured in a humidified atmosphere of $95 \%$ air and $5 \% \mathrm{CO}_{2}$ at $37{ }^{\circ} \mathrm{C}$ (Thermo Scientific, USA).

\subsubsection{Stability Studies}

The stability of NC3 and NC6 was monitored in 6 different biologically relevant solutions namely, $0.9 \%$ sodium chloride, $0.5 \%$ bovine serum albumin, $0.5 \%$ human serum albumin, $0.5 \%$ cysteine, $0.2 \mathrm{M}$ histidine, and $1 \times$ phosphate buffer saline. For the experiment, $800 \mu \mathrm{L}$ of nanoconstruct was mixed with $200 \mu \mathrm{L}$ of challenging solution and the UV-vis absorption of gold nanocages for recorded at 1 and $24 \mathrm{~h}$ time points. 


\section{Results for both NC3 and NC6 show high stability within the $24 \mathrm{~h}$ time point (Figure}

\section{4 and 15). Among both, PEGylated particles appear to be more soluble and stable with}

time.
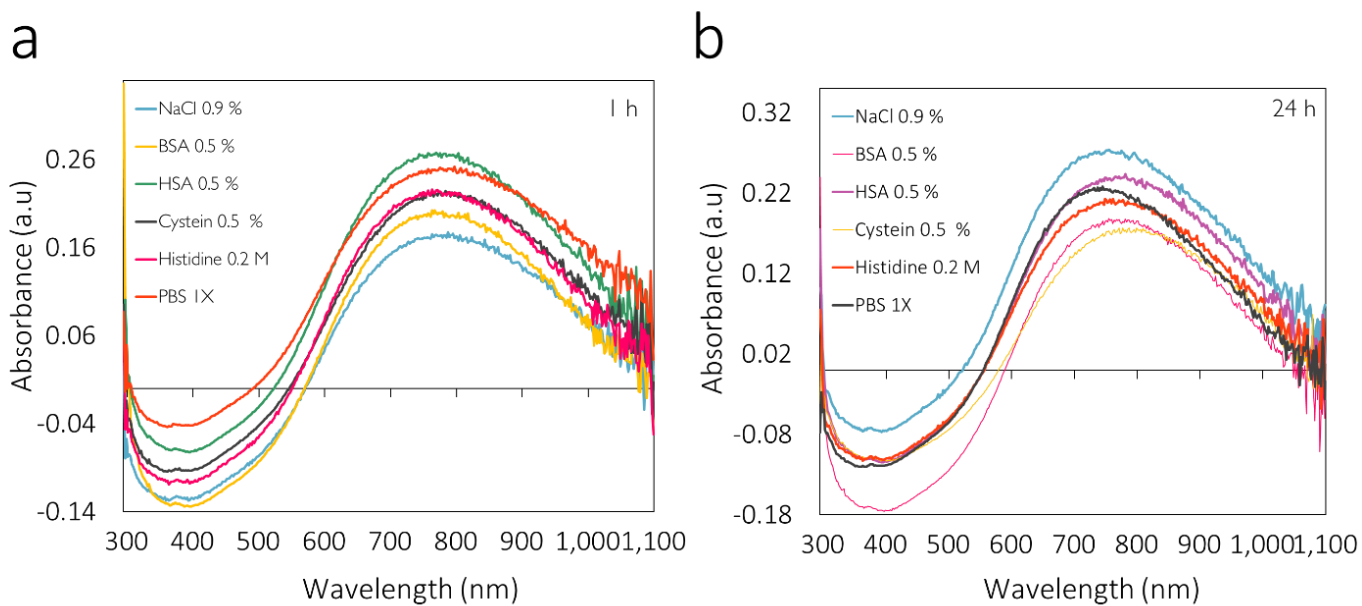

Figure 14. Stability of NC3 in different biologically relevant solutions with time was monitored using UV -visible spectroscopy. NC3 was treated with different solutions and plasmon absorption band monitored at (a) $1 \mathrm{~h}$ (b) $24 \mathrm{~h}$. Results indicate that nanocages are very stable even after $24 \mathrm{~h}$. However, aggregated cages were visibly observed in the vials.
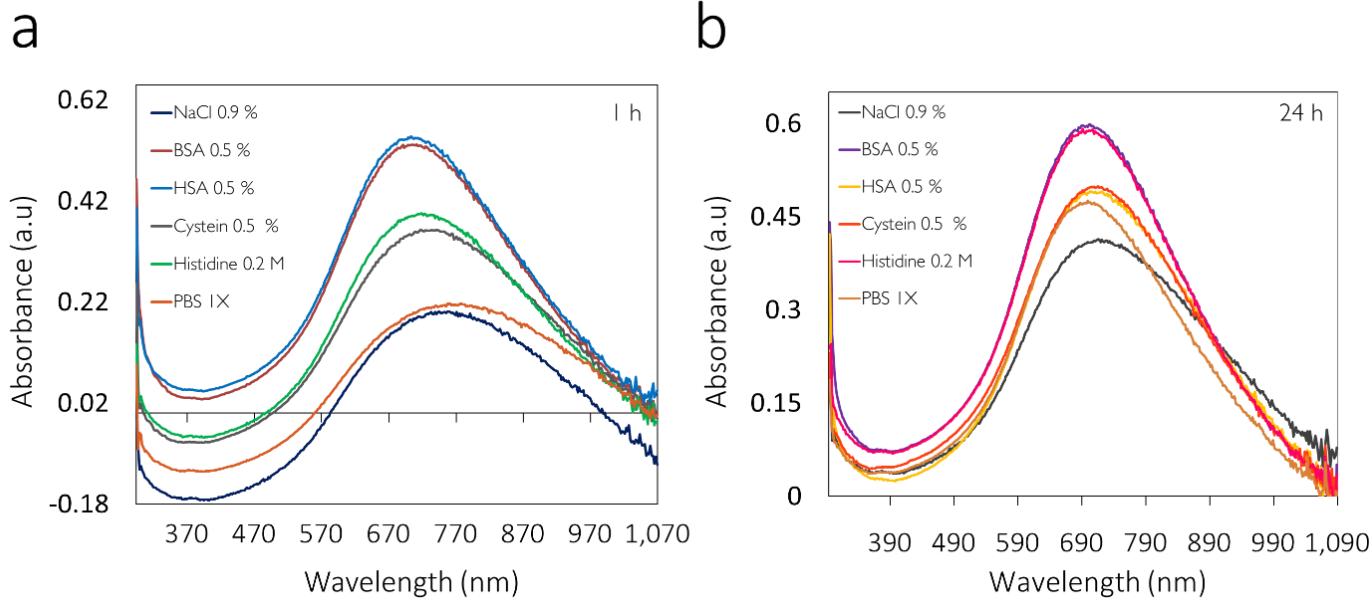

Figure 15. Stability of NC6 in different biologically relevant solutions with time was monitored using UV -visible spectroscopy. NC6 was treated with different solutions and plasmon absorption band monitored at (a) $1 \mathrm{~h}$ (b) $24 \mathrm{~h}$. Results indicate that nanocages are very stable even after $24 \mathrm{~h}$. No visible aggregation was observed as PEG coating improves the stability significantly. 


\subsubsection{MTT Assay}

MTT tests were performed for all nanoconstructs (NC1-NC6) to investigate the cytotoxicity in PC3 cells $\left(5 \times 10^{5}\right.$ cells $\left./ \mathrm{ml} ; 37^{\circ} \mathrm{C}\right)$. Cells $(70 \%$ confluence $)$ were seeded in flat bottom 96 -well polystyrene coated plates for $12 \mathrm{~h}$ at $37^{\circ} \mathrm{C}$ in a $\mathrm{CO}_{2}$ incubator. NC1-NC6 in media were prepared and tested for toxicity with the following concentrations: $2.5 \mu \mathrm{g} / \mathrm{mL}, 5 \mu \mathrm{g} / \mathrm{mL}, 10 \mu \mathrm{g} / \mathrm{mL}, 25 \mu \mathrm{g} / \mathrm{mL}$, and $50 \mu \mathrm{g} / \mathrm{mL}$. Each sample was tested in triplicates for statistical analysis. After $24 \mathrm{~h}$ incubation, $10 \mu \mathrm{L}$ per well MTT (stock solution $5 \mathrm{mg} / \mathrm{mL} 1 \times$ PBS) (ATCC, USA) was added and kept for $4 \mathrm{~h}$, and the formazan crystals so formed were dissolved in $100 \mu \mathrm{L}$ detergent/solubilizing buffer. The plates were kept for $2 \mathrm{~h}$ in the dark at $25^{\circ} \mathrm{C}$ to dissolve all crystals, and the intensity of developed color was measured using a Biotek Epoch plate reader operating at 570 nm wavelength.
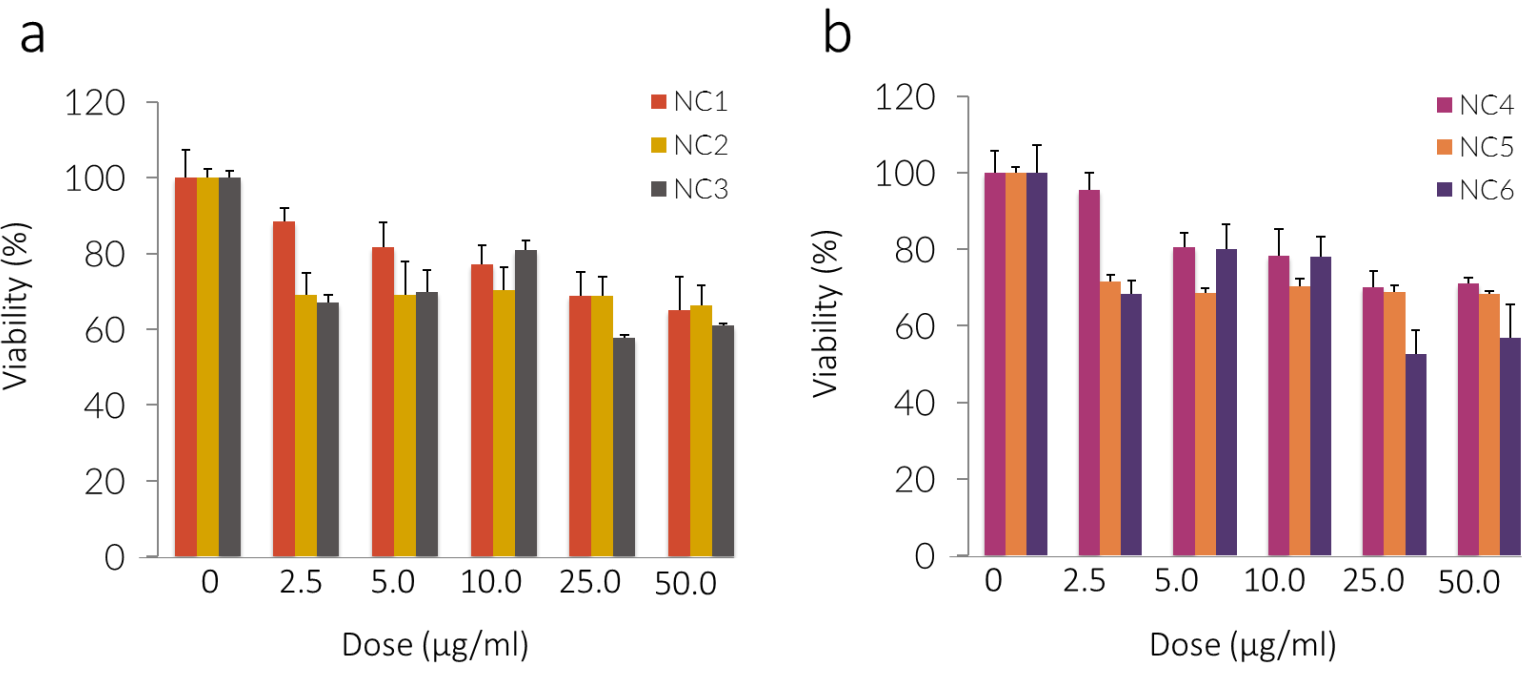

Figure 16. Stability of NC6 in different biologically relevant solutions with time was monitored using UV -visible spectroscopy. NC6 was treated with different solutions and plasmon absorption band monitored at (a) 1 h (b) 24 h. Results 
Wells with complete medium, nanoparticles, and MTT, but without cells, were used as blanks. Untreated cells were considered $100 \%$ viable. Intensities were then represented as dosage vs viability response plots. MTT assay results showed relative cell viability of $60 \%$ for higher doses up to $50 \mu \mathrm{g} / \mathrm{mL}$. It is evident from Figure 16 that nanoparticles with bombesin seem to be relatively toxic compared to BBN devoid counterparts. NC1 and NC4 controls show gradual decrease in viability, while NC2, NC3, NC5, and NC6 plateaus to $70-80 \%$ viability for all concentrations.

\subsubsection{Estimation of $\mathrm{IC}_{50}$ in PC3 cells}

Half maximal inhibitory concentration $\left(\mathrm{IC}_{50}\right)$ values were determined for $\mathrm{NC} 2, \mathrm{NC} 3$, NC5, and NC6 using radioactive competitive binding assays in PC3 cells. Specificity of nanocage to that of radioactive bombesin analogue ${ }^{125} \mathrm{I}-\mathrm{Ty}$ r4 $\mathrm{BBN}$ (GRPR specific peptide) was estimated to calculate the $\mathrm{IC}_{50}$. Approximately 30,000 cells were incubated at $37{ }^{\circ} \mathrm{C}$ for $40 \mathrm{~min}$ under $5 \% \mathrm{CO} 2$ in the presence of $20,000 \mathrm{cpm}{ }^{125} \mathrm{I}-\mathrm{Tyr} 4$ bombesin $(2200 \mathrm{Ci} / \mathrm{mmol})$. Along with the radioactive $\mathrm{BBN}$ analogue, nanoconstructs were coincubated in PC3 cells with increasing dosage. After incubation, the reaction medium was aspirated, and cells were washed three times with cold RPMI 1640 modified buffer. Cell bound radioactivity was then measured by a Packard Riastar $\gamma$ counter. $\mathrm{IC}_{50}$ values were calculated using GraphFit 4.0 graphing software to plot dosage response curves where values were represented in $\mu \mathrm{g} / \mathrm{mL}$. Each sample was tested in triplicate for statistical analysis (Figure 17) and final values were represented in $\mathrm{ng} / \mathrm{mL}$. 

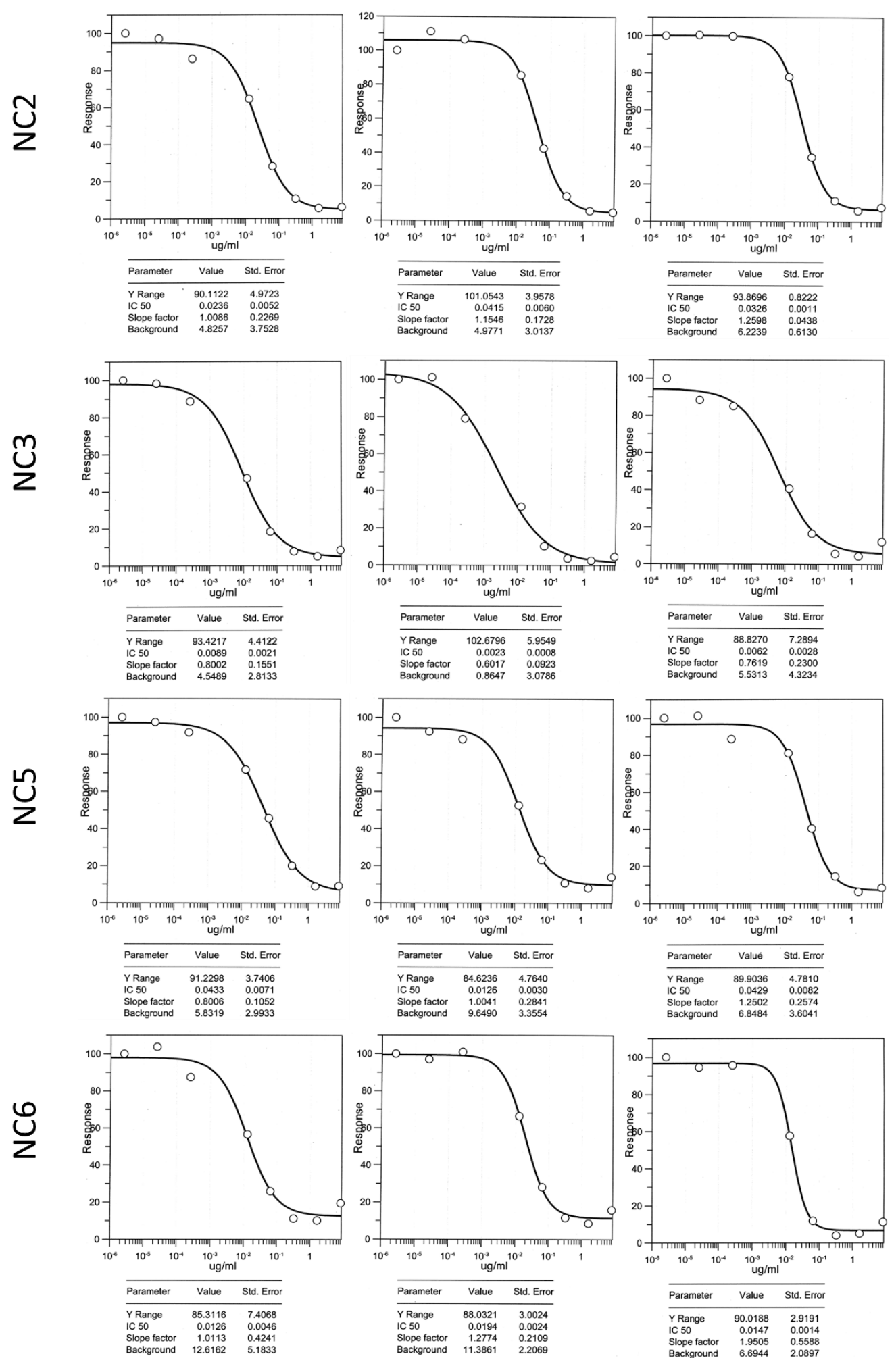

Figure 17. $\mathrm{IC}_{50}$ dosage response curves for nanoconjugates NC2, NC3, NC5 and NC6. 


\subsubsection{Prevention of non-specific binding to glass substrates}

For reducing nanoparticle binding to cell plate substrates and glass coverslips, $2 \mathrm{~mL}$ $0.01 \%$ poly-l-lysine solution was incubated on substrate surfaces in sterile conditions under UV light for $2 \mathrm{~h}$ prior experimental treatment. After incubation, poly-l-lysine solution was removed using a vacuum pump and substrate was rinsed once using sterile cell grade water. Poly-1-lysine coated wells/coverslips were then allowed to air-dry in sterile conditions for $4 \mathrm{~h}$ under UV light. After drying, plate wells/coverslips were used for cell seeding. Poly-l-lysine, a positively cationic polymer, was used to change the substrate surface charge and increase effective steric hindrance. The polymer coated slides did not reduce the cell adhesion or impart toxicity. Coating drastically reduced nonspecific binding of nanoparticles as compared to controls (Figure 18).

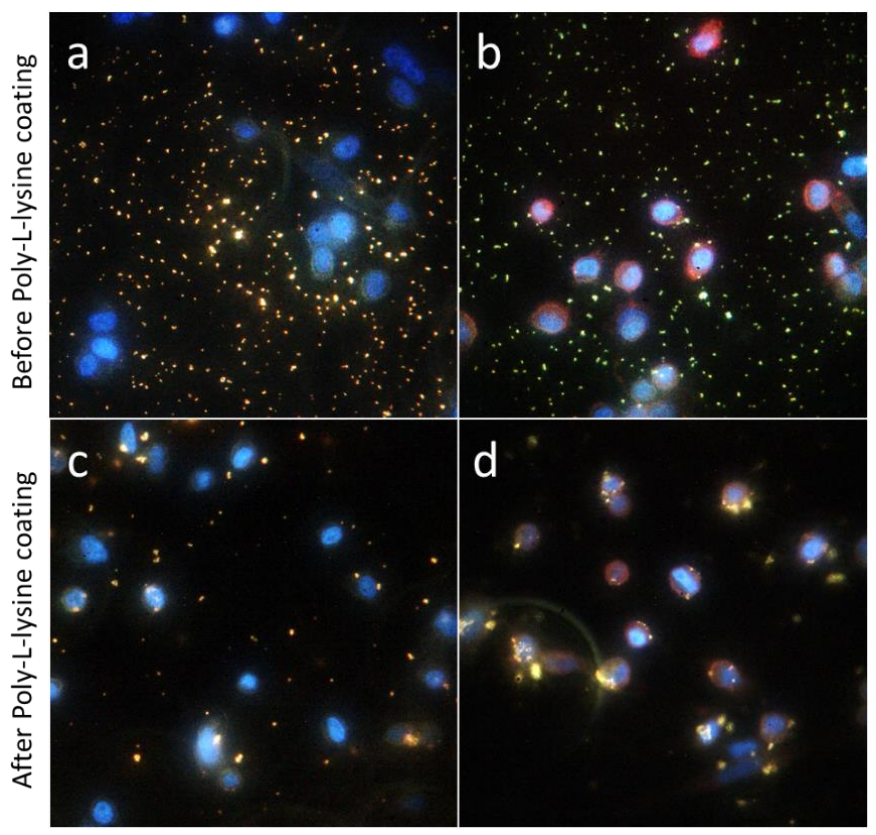

Figure 18. Non-specific binding of gold nanocages on the substrate was reduced by pretreatment with charged aminoacid homopolymers. (a-b) Prior to poly-L-lysine treatment, nanocages were seen everywhere on the glass slide along with PC3 cells; (c-d) After poly-L-lysine treatment, non-specific binding of nanocages to glass slide is visibly reduced. These images show poly-L-lysine coating on the substrate reduced non-specific binding of AuNCs. 


\subsubsection{Hyperspectral Imaging}

$2 \mu \mathrm{L}$ of dilute bombesin conjugated AuNCs was sandwiched between a clean glass slide and a coverslip. After drying for $10 \mathrm{~min}$ in a $40{ }^{\circ} \mathrm{C}$ hot air oven, the slide was focused under the dark field microscope at 100x. Cellular internalization of nanoparticles was detected using the Cytoviva dark field hyperspectral imager.

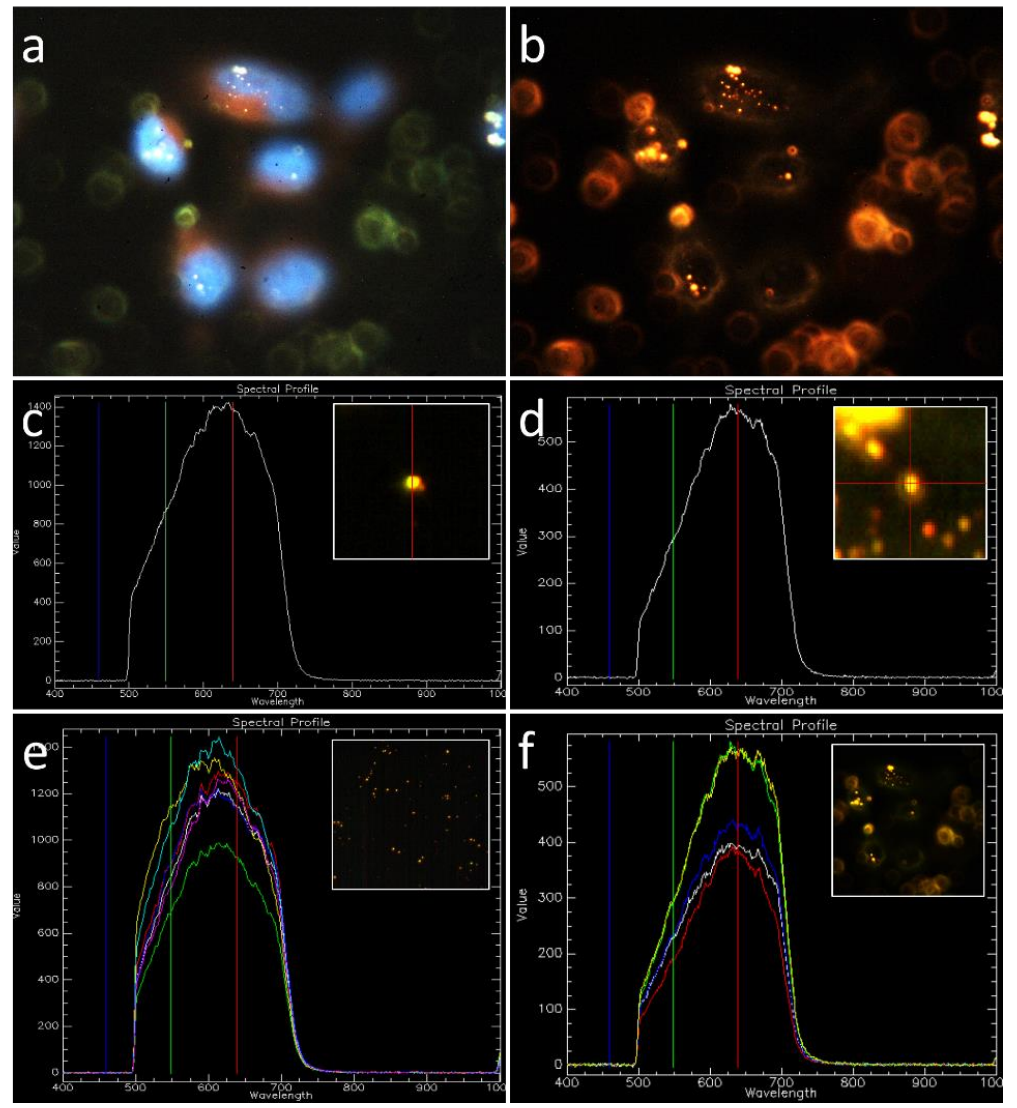

Figure 19. Construction of hyperspectral library for AuNC-BBN. (a) Dark field fluorescence image for bombesin conjugated AuNCs internalized in PC3 cells; (b) Corresponding hyperspectral image; (c) Spectral plot for a single Bombesin conjugated AuNC spot on a glass slide; (d) Spectral plot for a single Bombesin conjugated AuNC spot internalized within PC3 cells; (e) Hyperspectral library for Bombesin conjugated AuNCs coated on a glass slide with corresponding hyperspectral image; (f) Hyperspectral library for bombesin conjugated AuNCs internalized in PC3 cells Hyperspectral profile match confirms AuNC-BBN signals. Hyperspectral imaging of AuNC-BBN was not influenced in presence of cellular environment

Using ENVI imaging software, spectral profiles for AuNCs were recorded.

Hyperspectral profile of gold signals (or yellow spots) scattered by AuNCs in dark field 
without cell treatment were first recorded as control. Next hyperspectral of illuminated gold signals on cell treated glass coverslips were matched with controls. AuNCs were found to scatter light in the red band of the visible spectrum and a spectral library was constructed by recording profiles for multiple nanocages signals on the slides. Results evidently show similar profiles for bombesin conjugated AuNCs with and without cells were found (Figure 19).

\subsubsection{Pathway Blocking Studies using endocytic inhibitors}

Internalization of nanoconjugates in cells and their dependence on caveolae and clathrin mediated endocytosis were investigated using microscopy and image analysis. Briefly, NC1-NC6 were selectively studied in PC3 cells grown in 6 -well plates $\left(5 \times 10^{5}\right.$ cells/well) for following treatments: (a) cells without any inhibitors; (b) cells blocked for clathrin mediated endocytosis; (c) cells blocked for caveolae mediated endocytosis; (d) all modes of endocytosis blocked for cells treated at $4{ }^{\circ} \mathrm{C}$. Treatment concentrations in each well for clathrin blocking study were $10 \mu \mathrm{M}$ chlorpromazine (affects AP2 distribution and clathrin lattice assembly) and $2 \mu \mathrm{M}$ cytochalasin D (assists blocking actin function). Treatment concentrations in each well for caveolae blocking study were $5 \mathrm{mM}$ methyl- $\beta$-cyclodextrin (blocks lipid raft mediation via cholesterol depletion) and $100 \mu \mathrm{M}$ amiloride (blocks macropinocytosis via submembranous acidification). All samples were treated with inhibitors for 30 min followed by nanocage treatment (50 $\mu \mathrm{L}$ of $0.2 \mathrm{mg} / \mathrm{mL}$ ) for $2 \mathrm{~h}$ at $37{ }^{\circ} \mathrm{C}$. For energy dependent study (blocks all modes of endocytosis), cells were incubated for $30 \mathrm{~min}$ at $4{ }^{\circ} \mathrm{C}$, followed by nanocage treatment $\left(50 \mu \mathrm{L}\right.$ of $0.2 \mathrm{mg} / \mathrm{mL}$ ) for $2 \mathrm{~h}$ at $4{ }^{\circ} \mathrm{C}$ conditions. PC 3 cells not treated with nanoparticles 
were used as control. After $1 \mathrm{~h}$ treatment, cells were washed to remove unbound particles, and microscopic slides were prepared with DAPI nuclear stain. Slides were imaged using Cytoviva dark-field imaging (100× oil). Dark field imaging was utilized for investigating the cellular internalization of nanocages at both $37{ }^{\circ} \mathrm{C}$ and $4{ }^{\circ} \mathrm{C}$. Results show high internalization of NC3 and NC6 in PC3 cells at $37^{\circ} \mathrm{C}$, while none under $4{ }^{\circ} \mathrm{C}$ (Figure 20).

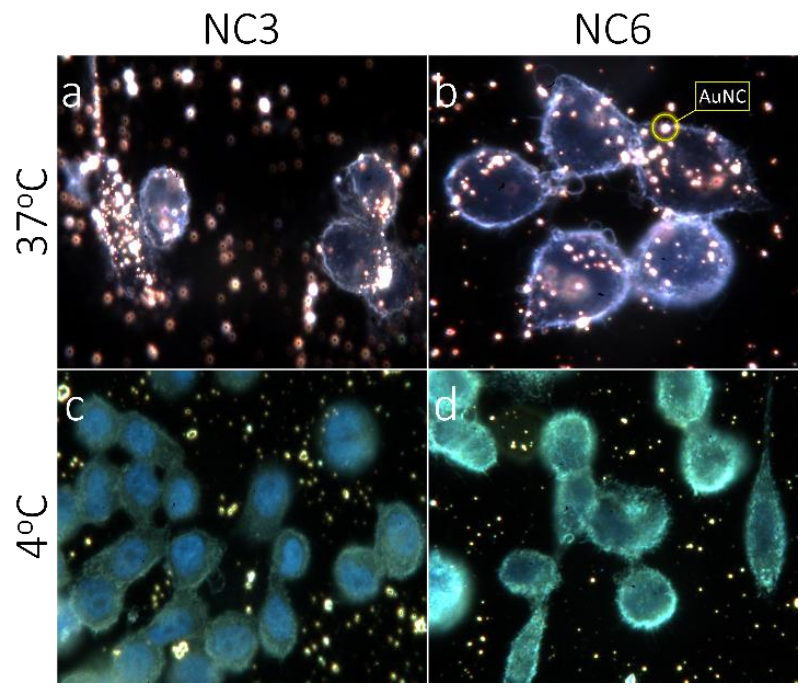

Figure 20. Dark field images of PC3 cells treated with nanoconjugates (a) NC3 after treatment with cells at $37^{\circ} \mathrm{C}$; (b) NC6 after treatment with cells at $37^{\circ} \mathrm{C}$; (c) $\mathrm{NC} 3$ after treatment with cells at $4{ }^{\circ} \mathrm{C}$ (d) NC6 after treatment with cells at $4{ }^{\circ} \mathrm{C}$. At $4{ }^{\circ} \mathrm{C}$ no internalization was observed as all endocytic processes were arrested. This result shows that nanocage internalization is energy dependent.

This result indicates an energy dependent pathway for cellular uptake of nanoparticles.

To understand the effect of surface coating on internalization, nanoconstructs NC1, NC3, NC4, and NC6 were studied for possible associations with caveolae and clathrin mediated endocytosis. Results show that NC3 and NC6 did not internalize after the clathrin pathway was blocked while it is seen that $\mathrm{NC} 1$ did not internalize after blocking the caveolae pathway (Figure 21). Images also show no uptake of PEGylated particles (NC4) within cells (Figure 21). 


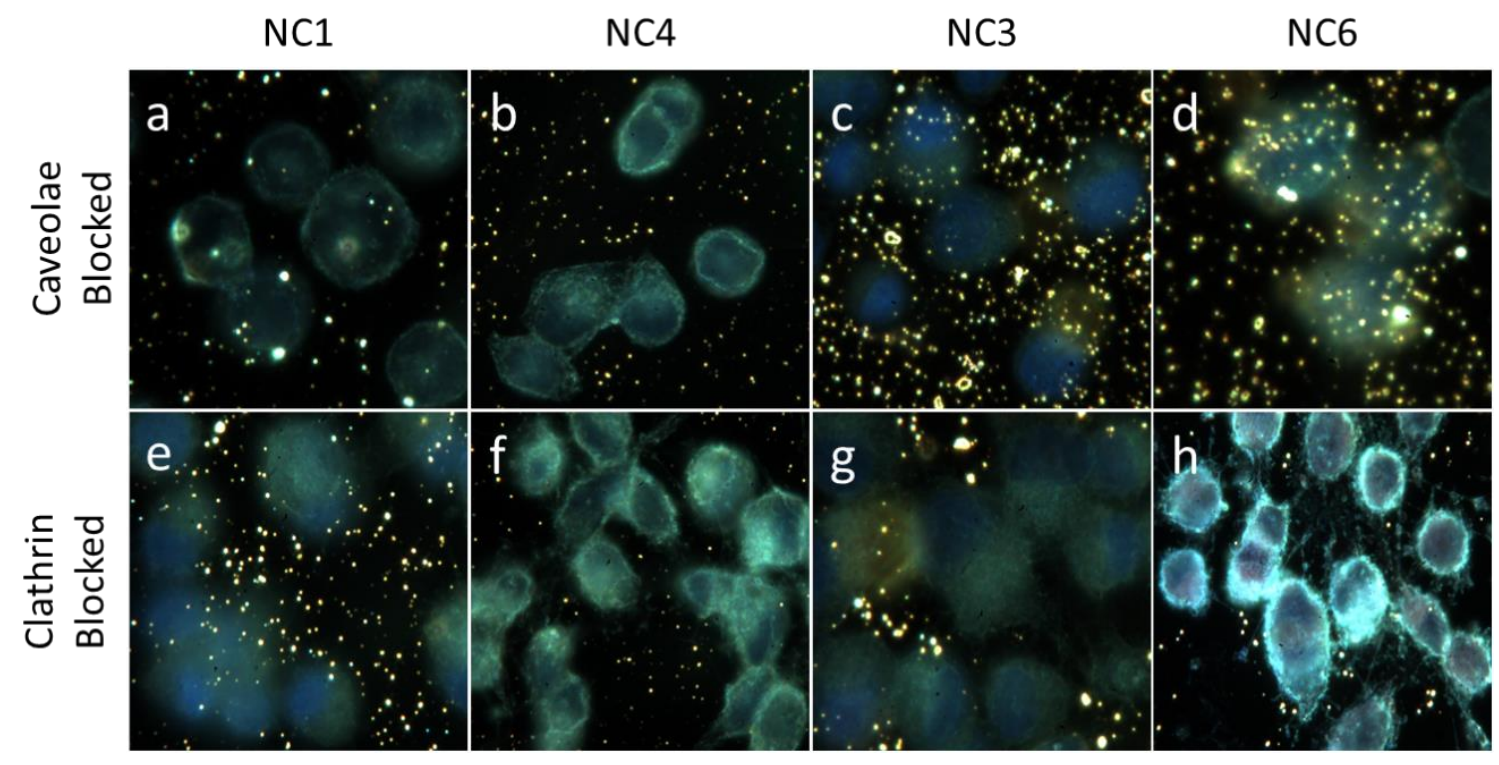

Figure 21. Dark field imaging for different type of surface coated nanoconjugates incubated with PC 3 cells, with specific endocytic pathways blocked using inhibitors. Top panel: Caveolae pathway blocked (a) NC1 on live cells (b) NC4 on live cells (c) NC3 on live cells (d) NC6 on live cells; Bottom panel: Clathrin pathway blocked (e) NC1 on live cells (f) NC4 on live cells (g) NC3 on live cells (h) NC6 on live cells. This result confirms that internalization of bombesin conjugated gold nanocages is dependent on clathrin pathway.

To investigate the mechanism of receptor mediated internalization NC6 was further studied in PC3 cells $\left(5 \times 10^{5}\right.$ cells/well $)$ grown in 6-well plates. In this study, certain endocytic pathways were selectively blocked using various inhibitors and uptake was investigated. (a) Cells without any inhibitor treatments were used as a live control. (b) To prove the uptake was indeed a dynamin dependent pathway (Caveolae or Clathrin mechanism), dynamin pathway was blocked prior NC6 treatment using dynasore hydrate $(80 \mu \mathrm{M})$. Results showed that uptake was affected in dynamin blocked cells. Therefore, clathrin mediated and caveolae mediated endocytosis were studied in PC3 cells as a next step. (c) Subsequently, caveolae pathway was blocked using methyl- $\beta$ cyclodextrin $(5 \mathrm{mM})$ or filipin $(5 \mu \mathrm{M})$. (d) Separately, Clathrin pathways were blocked using X-22 anti-clathrin antibody $(3 \mu \mathrm{g} / \mathrm{mL})$ or chlorpromazine $(20 \mu \mathrm{M})$ or pitstop-2 $(25 \mu \mathrm{M})$. After 30 min inhibitor treatment, inhibitor concentrations were halved in each 
well followed by NC6 $(10 \mu \mathrm{L}$ of $1 \mathrm{mg} / \mathrm{mL})$ treatment per well. After $4 \mathrm{~h}$ incubation at $37^{\circ} \mathrm{C}$, the resulting cells were washed to remove unbound nanocages, and microscopic slides were prepared with DAPI nuclear stain and CellMask Deep Red plasma membrane stain. All treatments were carried out in serum free media.

\subsubsection{Antibody mediated co-localized inhibition}

To study clathrin mediated endocytosis, X-22 anti-clathrin antibody was colocalized with NC6 in PC3 cells. The antibodies were purified by passing through a Zeba Micro spin desalting column to remove any preservatives. The antibody was treated with cells

with a slight modified procedure ${ }^{40}$. First, cells were incubated with PBS for 2 min at 37 ${ }^{\circ} \mathrm{C}$, followed by incubation with ice cold PBS for another 2 min. Cells were then incubated with permeabilization buffer $(0.01 \%$ Tween 20 in $1 \times \mathrm{PBS}$ at $\mathrm{pH} 7.6)$ for 1 min. The cells were washed with cold $1 \times$ PBS 3 times, and the antibody was treated in serum free media at $37{ }^{\circ} \mathrm{C}$ for $30 \mathrm{~min}$ followed by nanoconstruct treatment.

\subsubsection{Image analysis for NC6 quantification in cells}

NC6 aggregates were quantified from 200 cells from each blocking experiment. Each experiment was performed in duplicate for statistical analysis. The study included a live control, dynamin pathway blocked experiment (DYN-BLOCK, effectively blocking both clathrin and caveolae mediation) using dynasore, caveolae blocking using methyl- $\beta$-cyclodextrin $(\mathrm{CAV}-\mathrm{M} \beta \mathrm{CD})$, caveolae blocking using filipin (CAVFIL), clathrin blocking using X-22 anti-clathrin antibody (CLT-X22AB), clathrin 


\begin{tabular}{|c|c|c|c|c|c|c|c|c|c|c|c|c|c|}
\hline & \multicolumn{2}{|c|}{ LIVE } & \multicolumn{2}{|c|}{ DYN BLOCK } & \multicolumn{2}{|c|}{ CAV-MBCD } & & \multicolumn{2}{|c|}{ CAV-FIL } & \multicolumn{2}{|c|}{ CLT-CLOR } & \multicolumn{2}{|c|}{ CLT-PIT2 } \\
\hline No. & $\begin{array}{c}\text { SELECTED } \\
\text { CELL }\end{array}$ & $\begin{array}{l}\text { ASSOCIATED } \\
\text { AGGREGATES/ } \\
\text { CELL }\end{array}$ & $\begin{array}{l}\text { SELECTED } \\
\text { CELL }\end{array}$ & $\begin{array}{l}\text { ASSOCIATED } \\
\text { AGGREGATES/ } \\
\text { CELL }\end{array}$ & SELECTED CELL & $\begin{array}{l}\text { ASSOCIATED } \\
\text { AGGREGATES } \\
\text { /CELL }\end{array}$ & No. & $\begin{array}{l}\text { SELECTED } \\
\text { CELL }\end{array}$ & $\begin{array}{l}\text { ASSOCIATED } \\
\text { AGGREGATES } \\
\text { /CELL }\end{array}$ & $\begin{array}{l}\text { SELECTED } \\
\text { CELL }\end{array}$ & $\begin{array}{l}\text { ASSOCIATED } \\
\text { AGGREGATES } \\
\text { /CELL }\end{array}$ & $\begin{array}{l}\text { SELECTED } \\
\text { CELL. }\end{array}$ & $\begin{array}{l}\text { ASSOCIATED } \\
\text { AGGREGATES } \\
\text { /CELL }\end{array}$ \\
\hline 1 & & 31 & & 1 & & 48 & 1 & & 4 & & 0 & & 0 \\
\hline 2 & & 38 & & 4 & & 11 & 2 & & 5 & & 0 & & 5 \\
\hline 3 & & 10 & & 6 & & 20 & 3 & & 9 & & 0 & & 1 \\
\hline 4 & & 23 & & 0 & & 31 & 4 & & 7 & & 0 & & 0 \\
\hline 5 & & 24 & & 3 & $\$$ & 8 & 5 & & 3 & & 0 & & 4 \\
\hline 6 & & 29 & & 9 & & 28 & 6 & & 13 & & 5 & & 0 \\
\hline 7 & & 12 & & 8 & $\theta$ & 48 & 7 & & 11 & & 1 & & 4 \\
\hline 8 & & 22 & & 1 & & 11 & 8 & & 9 & & 2 & & 12 \\
\hline 9 & & 40 & & 0 & & 7 & 9 & & 13 & & 7 & & 2 \\
\hline 10 & & 2 & & 4 & & 5 & 10 & 48 & 10 & & 0 & & 9 \\
\hline
\end{tabular}

Table 1. Cells were exposed to NC6 after specific endocytic pathways were blocked using inhibitors. Visible gold signals from 100 individual cells were collected for analysis. Only representative images are provided here. (LIVE) Cells exposed to NC6 without pre-treatment of inhibitors were used as control. (DYN-BLOCK) Dynamin pathway blocked using dynasore, (CAV-M $\beta C D$ ) caveolae pathway blocking using methyl- $\beta$-cyclodextrin, (CAV-FIL) caveolae pathway blocking using filipin, (CLT-CLOR) clathrin pathway blocking using chlorpromazine, and (CLT-PIT2) clathrin pathway blocking using pitstop-2

blocking using chlorpromazine (CLT-CLOR), and clathrin blocking using pitstop-2 (CLT-PIT2). Individual cells were analyzed for NC6 uptake as shown in Table 1. Data from duplicates averaged and analyzed for quantification (Table 2). 


\begin{tabular}{|c|c|c|c|c|c|c|c|c|}
\hline \multicolumn{8}{|c|}{$\begin{array}{l}\text { RESULTS SUMMARY: NC6 ACCUMULATION IN } 20 \\
\text { Statistical analysis result from duplicates. All results have been averaged. } \\
\text { *Each visible gold signal, i.e. nanoaggregate is approximated as } 1 \text { unit }\end{array}$} & \multirow[b]{2}{*}{ Trend } \\
\hline & UNT & DYN & CAV-MßCD & CAV-FIL & CLT-X22AB & CLT-CLOR & CLT-PIT2 & \\
\hline Total Nanoaggregate uptake in 200 cells & 4000 & 836 & 3069 & 3023 & - 751 & - 561 & - 564 & \\
\hline SD & 428.51 & 98.47 & 319.39 & 190.00 & 157.21 & 70.70 & 27.72 & \\
\hline SE & 303.00 & 69.63 & 225.84 & 134.35 & 111.16 & 50.00 & 19.60 & \\
\hline Average Nanoaggregate/Cell & 21 & 4 & 16 & 16 & 4 & 3 & 3 & \\
\hline SD & 2.14 & 0.07 & 3.34 & 0.61 & 0.43 & 0.07 & 0.45 & \\
\hline SE & 1.52 & 0.05 & 2.36 & 0.43 & 0.31 & 0.05 & 0.32 & \\
\hline Empty Cells & 2 & 59 & 11 & 8 & 87 & 90 & 82 & $\sim$ \\
\hline SD & 1.41 & 0.00 & 3.54 & 3.54 & 14.14 & 26.87 & 12.02 & \\
\hline SE & 1.00 & 0.00 & 2.50 & 2.50 & 10.00 & 19.00 & 8.50 & \\
\hline \% REDUCTION & $0 \%$ & $-79 \%$ & $-23 \%$ & $-24 \%$ & $-81 \%$ & $-86 \%$ & $-86 \%$ & \\
\hline
\end{tabular}

Table 2. Quantification of AuNC-BBN within PC3 cells using optical microscopy (dark field fluorescence illumination).

NC6 was quantified as nanoaggregates within cells. (UNT) Cells exposed to NC6 without pre-treatment of inhibitors; (DYN) dynamin pathway blocked using dynasore; (CAV-M $\beta C D)$ caveolae pathway blocking using methyl- $\beta$-cyclodextrin;

(CAV-FIL) caveolae pathway blocking using filipin; (CLT-X22AB) clathrin pathway inhibition using anti-clathrin antibody colocalization (CLT-CLOR) clathrin pathway blocking using chlorpromazine; and (CLT-PIT2) clathrin pathway blocking using pitstop-2. Results show decrease in AuNC-BBN uptake when CME was blocked.

Using the data, the mechanism of internalization is explained through series of endocytic steps representations as schemes. The molecules illustrated in these schematic can be identified with a key glossary as shown in Figure 22.

\subsubsection{Quantification of nanoparticle uptake using Neutron Activation Analysis}

Amount of nanoparticles in cytoplasm of cells was quantified as total gold content using Neutron activation analysis (NAA). In this study, NC1, NC3, and NC6 were treated in PC3 cells $\left(4.25 \times 10^{5}\right.$ cells/well $)$ grown in 6-well plates. After blocking clathrin mediated or caveolae mediated endocytosis, internalization within cells were compared. Treatment concentrations in each well for clathrin blocking study were 10 $\mu \mathrm{M}$ chlorpromazine and 


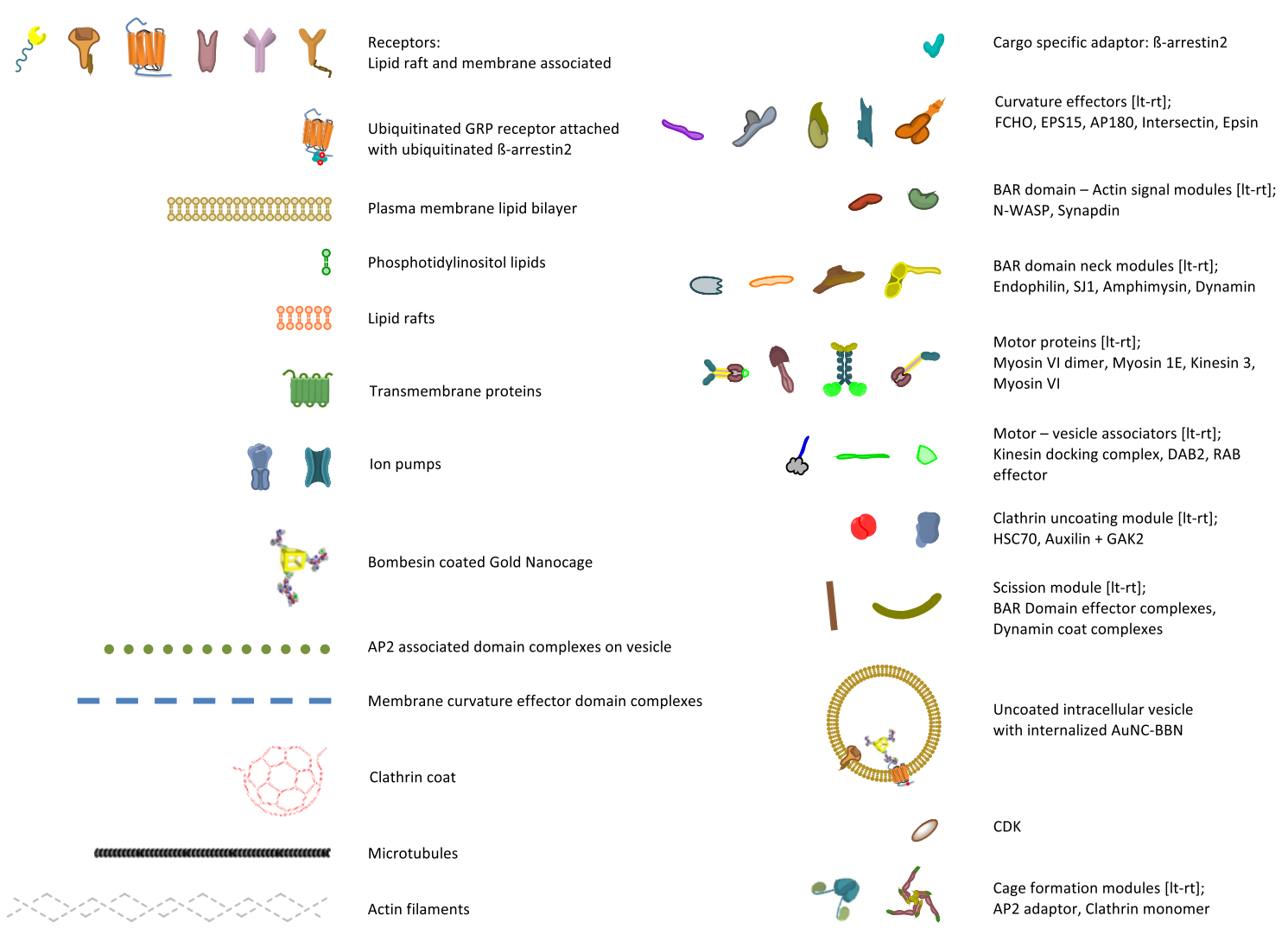

Figure 22. Glossary for schematics showing 2-D representation of the cell membrane during CME.

$2 \mu \mathrm{M}$ cytochalasin D. Treatment concentrations in each well for caveolae blocking study were $5 \mathrm{mM}$ methyl- $\beta$-cyclodextrin and $100 \mu \mathrm{M}$ amiloride. For gold internalization control, cells were not incubated with nanoparticles. After $30 \mathrm{~min}$ inhibitor treatment, PC3 cells were treated with $50 \mu \mathrm{L}$ of nanoconstruct to achieve a gold concentration of $13.35 \mathrm{ppm} /$ well. After $4 \mathrm{~h}$ incubation at $37{ }^{\circ} \mathrm{C}$, resulting cells were washed, dislodged, and stored as cell pellets at $-20{ }^{\circ} \mathrm{C}$ for NAA analysis for measuring varying levels of gold with respect to internalization in samples. NAA analysis was conducted at a Missouri University Research Reactor (MURR). Briefly, cell samples were dried, weighed and placed in high density polyethylene irradiation vials. The vials were irradiated inside the nuclear reactor core for less than a minute in 
a thermal flux density of $\sim 5 \times 10^{13} \mathrm{ncm}^{-2} \mathrm{~s}^{-1}$. After a decay time of $24 \mathrm{~h}$, the $\beta^{-}$radiation (411 KeV gamma ray) of ${ }^{198} \mathrm{Au}$ was measured using a high purity germanium detector (HPGe) for $1 \mathrm{~h}$ at a sample gap length of $5 \mathrm{~cm}$. Experiments were performed in triplicates for statistical analysis and data was represented as comparative percentages of gold (ppm). Preliminary analysis for NC3 and NC6 showed an insignificant 20\% decrease in internalization for clathrin pathway relative to caveolae pathway after blocking. This was caused due to nonspecific binding of AuNCs on substrate that led to erroneous results. Even after multiple washes using PBS or acidic buffers results did not yield any desirable outcome. This issue has been previously documented in another study ${ }^{41}$.

To overcome the problem of nonspecific substrates were coated with poly-1lysine. Cells collected after treatment was then subjected to cell sorting using flow cytometry (Figure 23). For this study the experiments were planned as follows: (a) untreated control - without any inhibitors; (b) colocalization with $3 \mu \mathrm{g} / \mathrm{mL} \mathrm{X-22} \mathrm{anti-}$ clathrin antibody; (c) pretreatment with clathrin inhibiting chlorpromazine. Results were then corroborated with data obtained with dark-field microscopy analysis. All treatments were carried out in serum free media. 


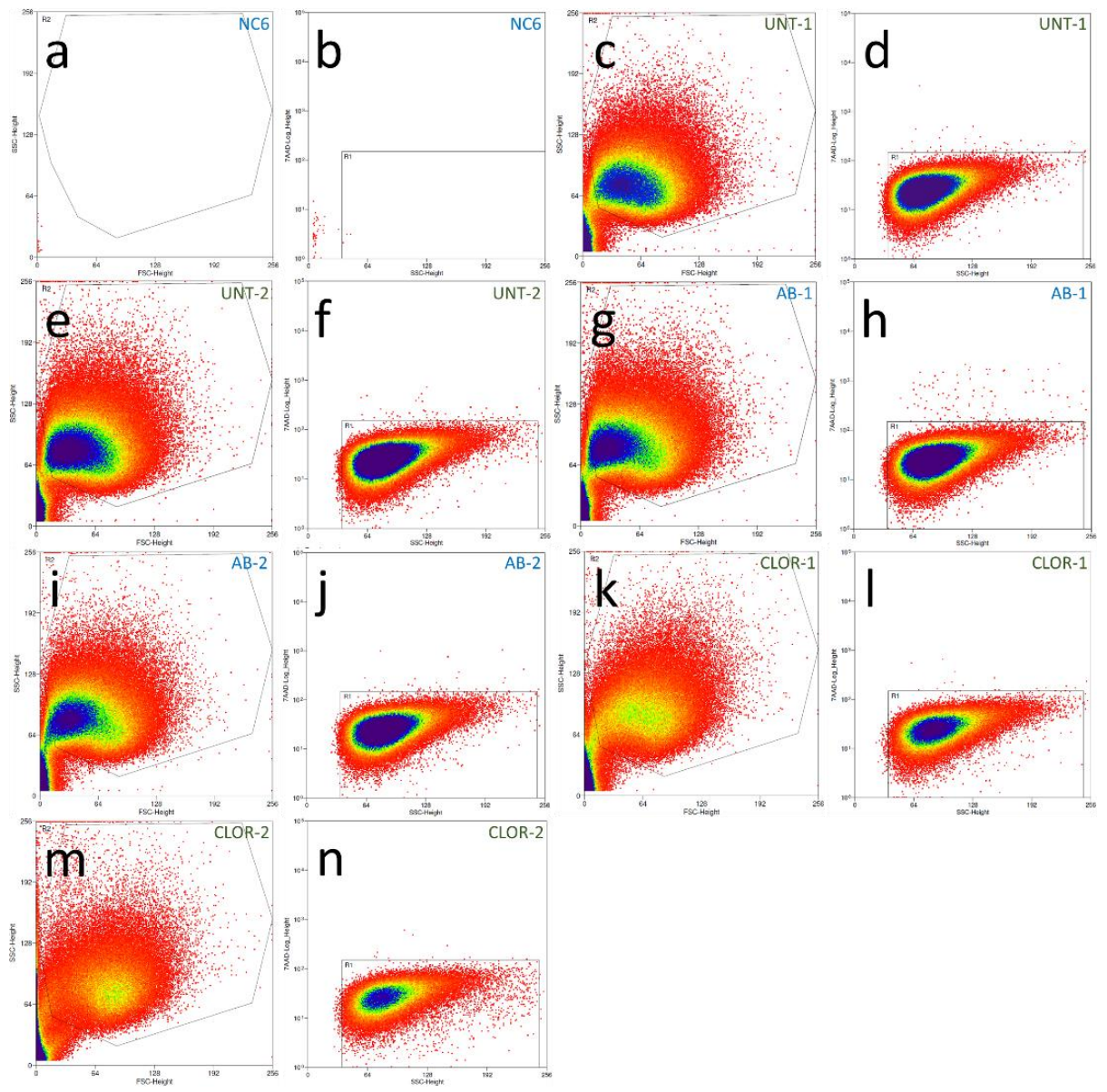

Figure 23. Flow cytometry dot plots showing FSC and SSC. For cell samples, R2 represents gate to sort PC3 cells from total registered events. Nanocage events falling outside the gate (based on control FCS and SSC for NC6) are excluded from sort. R1 represents gate to sort live cell events negative for 7AAD into collection tube. (a-b) NC6 in media for control plot; (c-f) UNT: Duplicate experiments for NC6 treated PC3 cells pretreated with no inhibitors; (g-j) AB: Duplicate experiments for NC6 treated PC3 cells pretreated with X-22 anti-clathrin antibody; (k-n) Duplicate experiments for NC6 treated PC3 cells pretreated with clathrin pathway inhibitor chlorpromazine.

\subsubsection{TEM analysis for NC6 uptake in cells}

TEM was utilized to document several CME characteristic processes such as clathrin coated pits, vesicular budding and presence of clathrin coated vesicles (CCVs). Results show formation of pits in cellular membrane of PC3 cells following nanoparticle treatment (Figure 24). Subsequently, images document dynamin dependent vesicular formation with characteristic tightening of the neck and presence of nascent budded vesicles (Figure 25). 

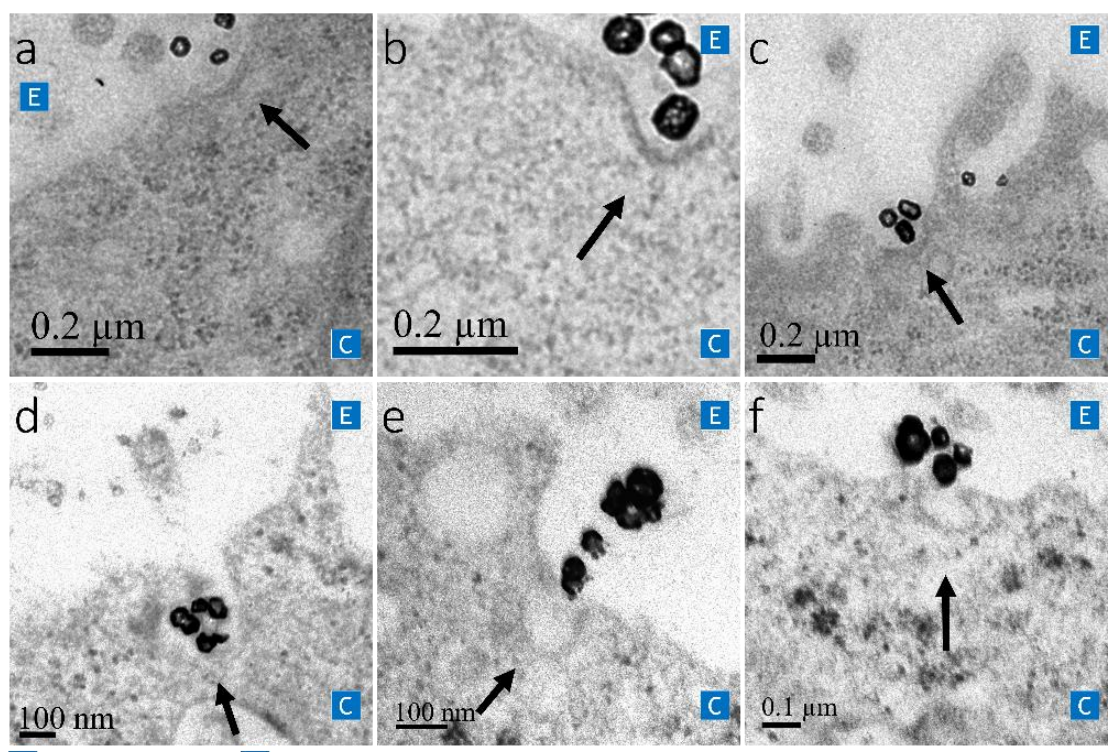

E Extracellular space; C Cytoplasm

Figure 24. TEM images showing plasma membrane of PC3 cells after exposure to bombesin conjugated gold nanocages. (a-f) Clathrin coated pit formation associated with endocytosis of NC6.

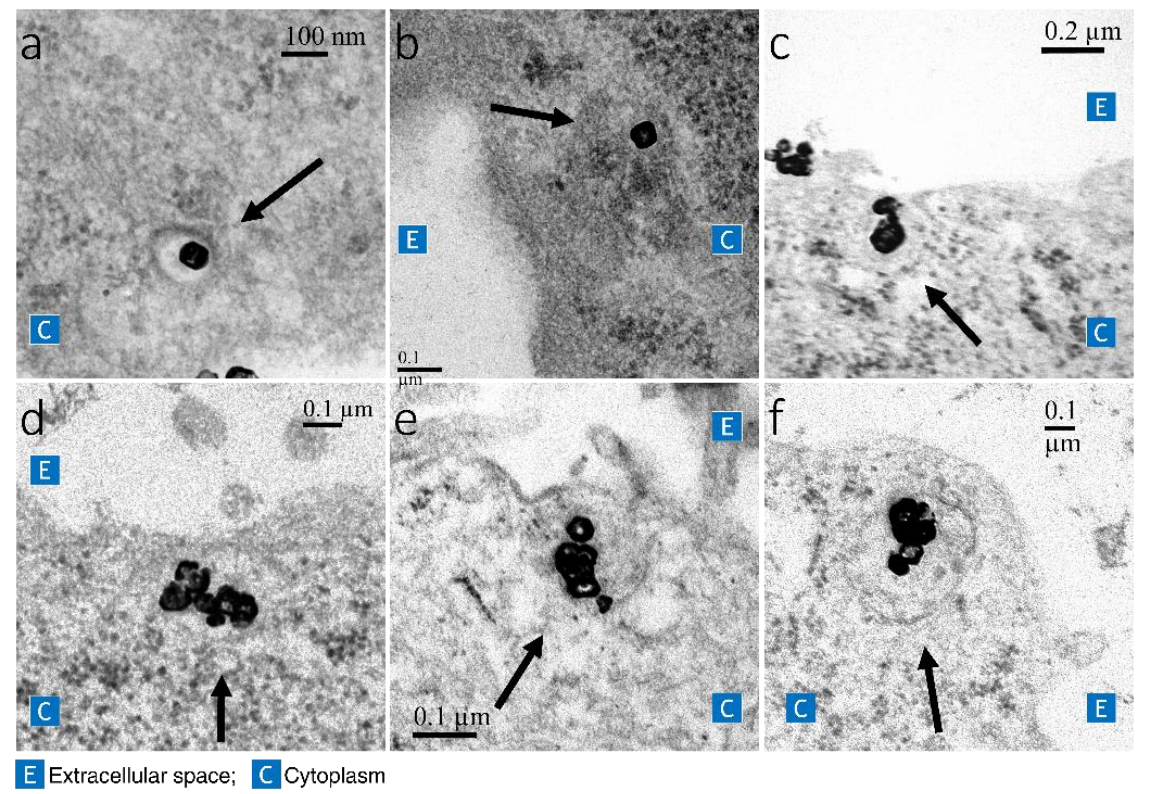

Figure 25. TEM images showing plasma membrane of PC3 cells after exposure to bombesin conjugated gold nanocages. (a-f) The transition of invaginations to form budded clathrin coated vesicles in the cytoplasm was observed. 
Following budding, nanoparticles are seen tethered to the inner membrane of a CCV or early endosomes (Figure 26) followed by late stage accumulation in endosomes or lysosomes. Results show presence of AuNCs in the cytoplasmic matrix indicating late endosomal or lysosomal release due to vesicular rupture within the cell (Figure 26).

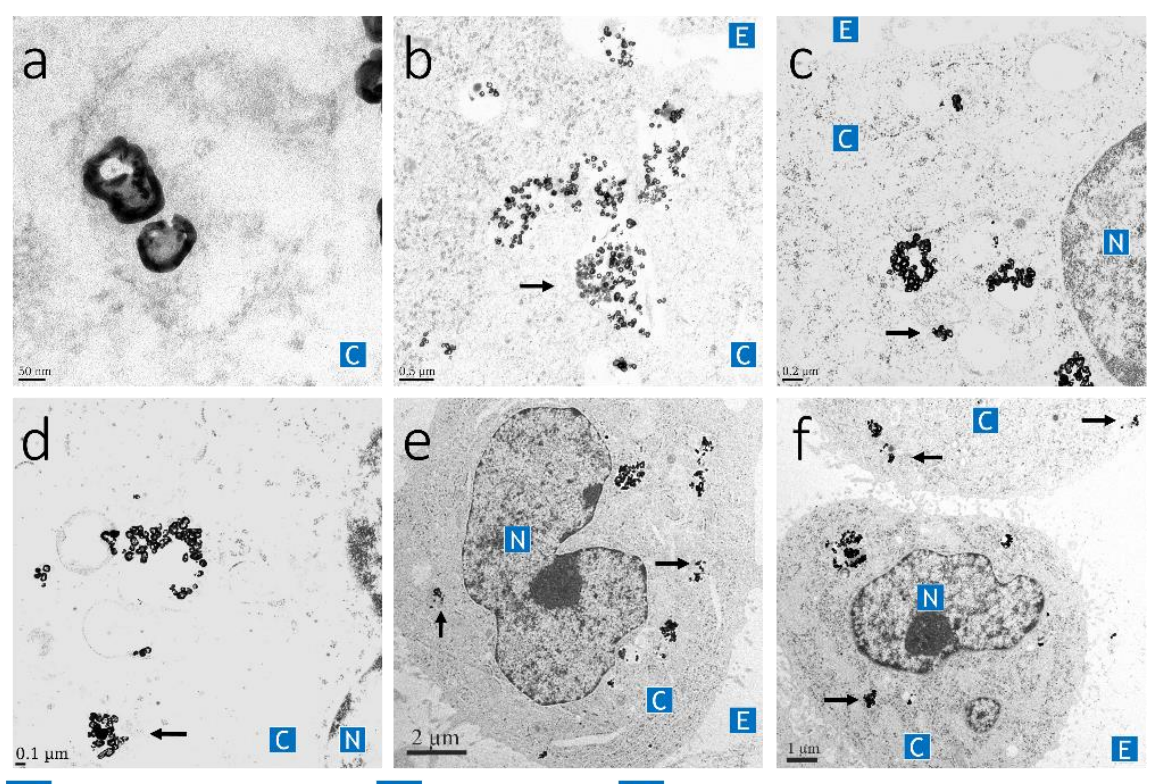

E Extracellular space; C Cytoplasm; N Nucleus

Figure 26. TEM images showing trafficking of AuNC-BBN into endosomal vesicles and lysosomes. (a) NC6 still attached to the GRP receptor on the inner side of clathrin coated vesicle; (b-c) AuNCs final fate in lysosomes. AuNCs exit the lysosomes and enter into cytoplasmic cellular space.

\subsubsection{Statistical analysis}

(a) Microscopy: For the analysis, we denoted the inhibitor treatments as CLT, DYN and CAV. Statistically significant separation of one of the groups is shown in (Figure 27 and Table 3). However, due to the very small sample size different tests were performed to estimate the correlation. When comparing only CLT and CAV, we used the Wilcoxon Rank Sum test. When comparing all three inhibitors, a Kruskal Wallis test was used. Both tests indicated that the CAV gives significantly higher results than 
CLT with a p-value of 0.0048 (assuming a one-sided alternative is appropriate). The difference was not statistically significant for DYN even though the mean and median values were largely different. This result can be attributed to small observation size for DYN (duplicates).
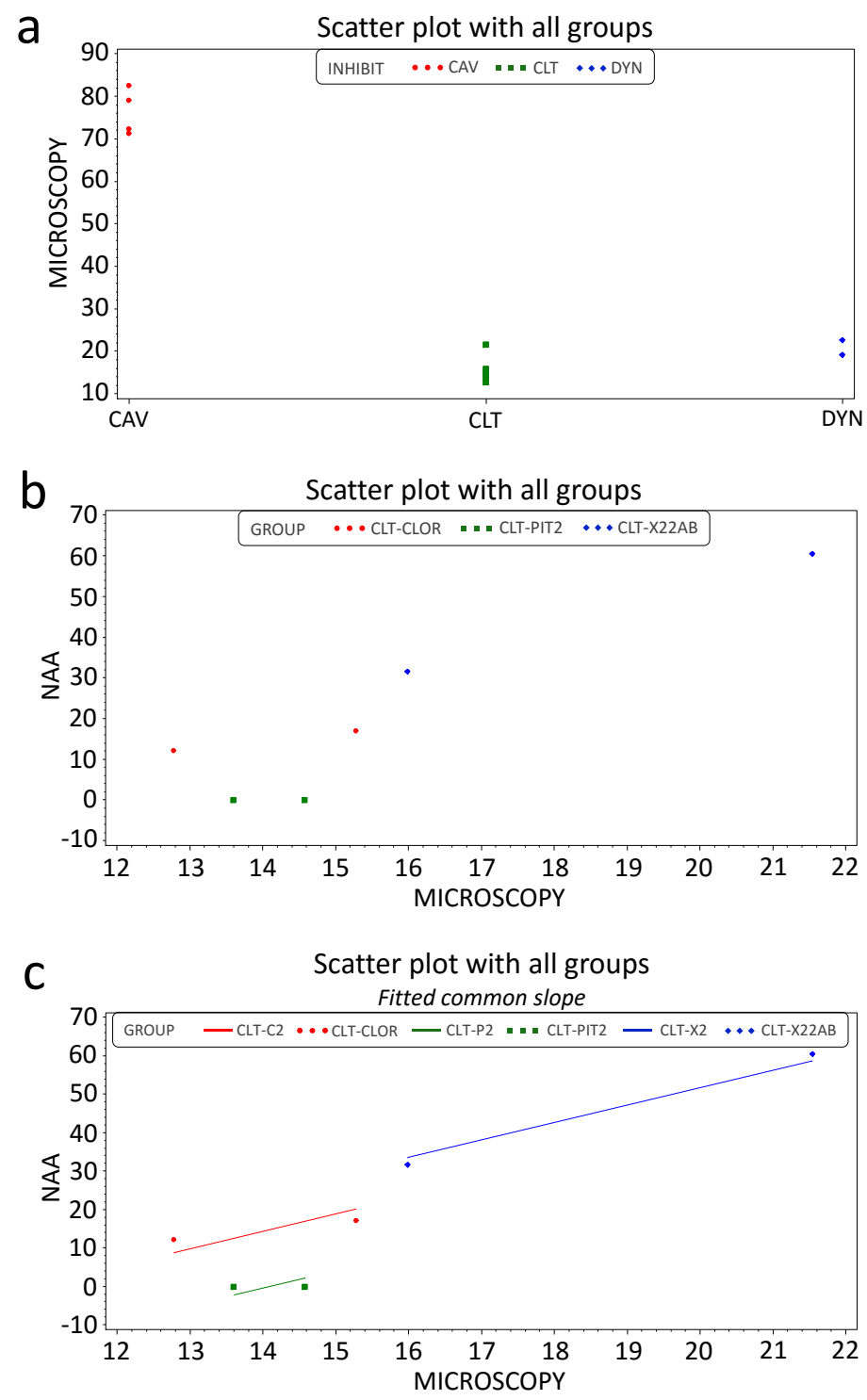

Figure 27. Statistical analysis on internalization of NC6 in PC3 cells in presence of inhibitors. 
(a)

\begin{tabular}{cccccc}
\hline Inhibitor & $\mathbf{N}$ & $\begin{array}{c}\text { Sum of } \\
\text { scores }\end{array}$ & $\begin{array}{c}\text { Expected } \\
\text { under H0 }\end{array}$ & $\begin{array}{c}\text { Std Dev } \\
\text { under Ho }\end{array}$ & $\begin{array}{c}\text { Mean } \\
\text { Score }\end{array}$ \\
\hline CLT & 6 & 22.0 & 39.0 & 6.244998 & 3.666667 \\
\hline DYN & 2 & 14.0 & 13.0 & 4.654747 & 7.000000 \\
\hline CAV & 4 & 42.0 & 26.0 & 5.887841 & 5.887841 \\
\hline
\end{tabular}

(b)

\begin{tabular}{ccc}
\hline & Chi-Square & 8.6667 \\
\hline & DF & 2 \\
\hline Asymptotic & Pr $>$ Chi-Square & 0.0131 \\
\hline Exact & Pr $>$ = Chi-Square & 0.0010 \\
\hline
\end{tabular}

(c)

\begin{tabular}{cccc}
\hline Inhibitor & Wilcoxon $\mathbf{Z}$ & DSCF value & Pr $>$ DSCF \\
\hline CLT vs. & -1.6667 & 2.3570 & 0.2181 \\
DYN & & & 3.6181 \\
\hline CLT vs. & -2.5584 & & $\begin{array}{c}0.0284 \text { (P-value adjusted } \\
\text { for multiple comparisons) }\end{array}$ \\
CAV & & 2.6186 & 0.1530 \\
\hline DYN vs. & -1.8516 & & \\
CAV & & & \\
\hline
\end{tabular}

(d)

\begin{tabular}{cccccc}
\hline Inhibit & $\mathbf{N}$ & $\begin{array}{c}\text { Sum of } \\
\text { scores }\end{array}$ & $\begin{array}{c}\text { Expected } \\
\text { under Ho }\end{array}$ & $\begin{array}{c}\text { Std Dev } \\
\text { under Ho }\end{array}$ & Mean Score \\
\hline CLT & 6 & 21.0 & 33.0 & 4.690416 & 3.50 \\
\hline CAV & 4 & 34.0 & 22.0 & 4.690416 & 4.690416 \\
\hline
\end{tabular}

(e)

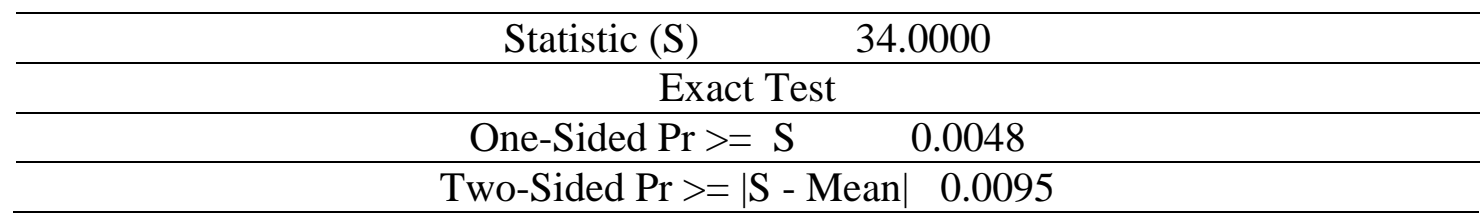

Table 3. Statistical Analysis. (a) Wilcoxon Scores (Rank Sums) for Variable microscopy Classified by Variable inhibitors; (b) Kruskal-Wallis test; (c) Pairwise Two-Sided Multiple Comparison Analysis Dwass, Steel, Critchlow-Fligner Method; Variable: microscopy; (d) Wilcoxon Scores (Rank Sums) for Variable microscopy Classified by Variable Inhibitors; and (e) Wilcoxon Two-Sample Test. 
(b) Correlation of microscopy with NAA data: Since it will not be appropriate to pool all six observations and treat as one single sample for the sake of small observation size, we utilized a method suggested by Bland and Altman ${ }^{42}$. Using this method, we assume a common correlation between microscopy and NAA counts for each inhibitor treatment. The analysis of covariance with the correlation estimate were obtained by partitioning sums of squares. The data is represented by a scatterplot of the six data points and separately with scatter plot along with the estimated regression line (based on covariance model) for each inhibitor included (Figure 27).

The estimated correlation using this method is 0.9556 . However, the test for the correlation being 0 is only marginally significant $(\mathrm{p}=0.0444)$ due to smaller observation size. Although this method of estimating a correlation does not lend itself to finding a confidence interval estimate for the correlation, given the observed p-value one would conjecture that a $95 \%$ confidence interval estimate should range from 0.02 to 0.99 . Based on the width of this confidence interval the estimate can be considered rough. However, the evidence does indicate the presence of a positive correlation between NAA and microscopy.

\subsection{RESULTS AND DISCUSSIONS}

\subsubsection{Properties of BBN conjugated gold nanocages}

First, AuNCs with PVP were synthesized (NC1). Then 2 types of conjugates were synthesized based on surface polymer chemistry, (1) AuNC-BBN-PVP wherein PVP (NC2 and NC3) was an additional polymer ionically linked to the surface to impart stability and (2) AuNC-BBN-PEG wherein PEG (NC5 and NC6) was an additional 
polymer covalently linked to the surface to impart stability (Scheme 1). Here NC2 and NC3 and similarly NC5 and NC6 had different ratio of peptide attached to surface. As PVP was ionically linked we hypothesized that more amounts of thioctic acid-BBN would displace the polymer and attach to the surface as compared to PEG. All nanoconstructs NC1-NC6 had a UV max absorption peak at 700-750 nm, indicating no changes to particle stability (Table 4). NC1 and NC4 devoid of bombesin had a negative zeta potential while other constructs conjugated to bombesin had a positive zeta value (Table 4). The increase in zeta value was indicative of amount of bombesin displaced on the surface.

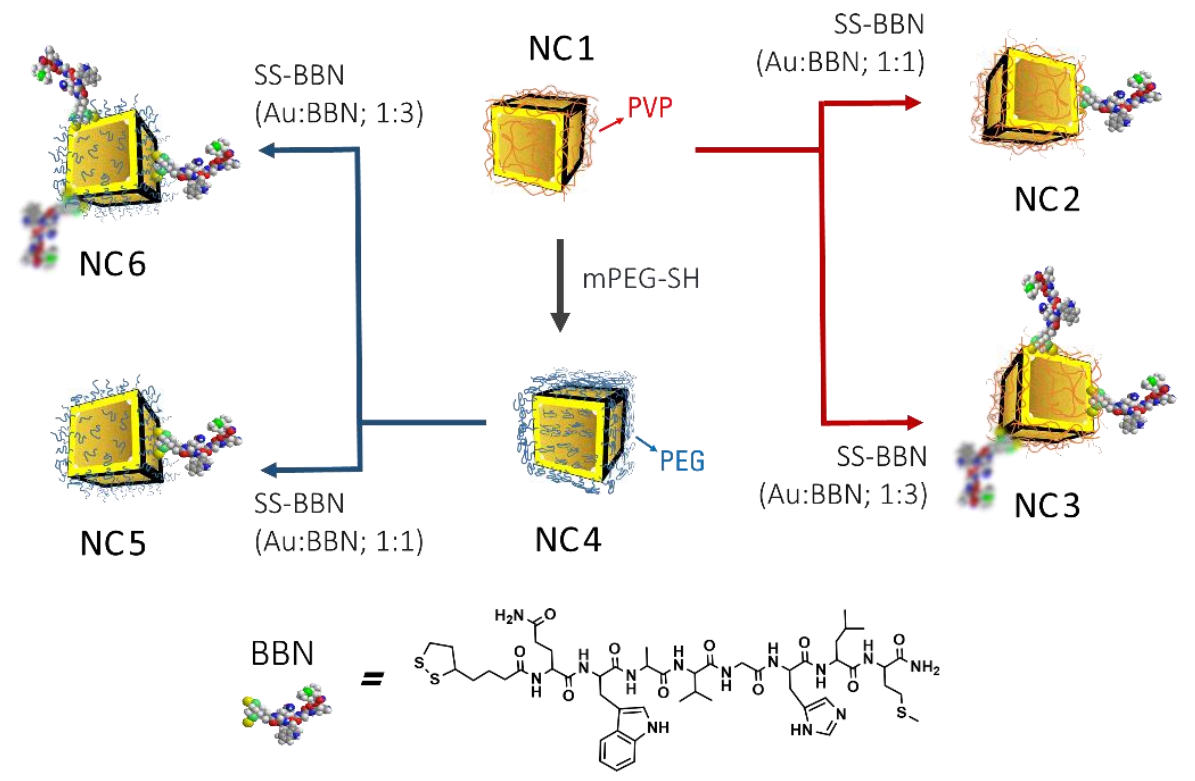

Scheme 1. Schematic Illustration of the Synthesis of Bombesin Conjugated Gold Nanocages. 


\begin{tabular}{cccccc}
$\begin{array}{c}\text { Conjugate } \\
\text { code }\end{array}$ & Conjugate Name & Au:BBN & $\zeta(\mathrm{mV})$ & $\begin{array}{c}\text { Hydrodynamic } \\
\text { size }(\mathrm{nm})\end{array}$ & $\begin{array}{c}I \mathrm{C}_{50} P C 3 \\
(\mathrm{ng} / \mathrm{ml})\end{array}$ \\
\hline NC1 & AuNC-PVP & Control & -35.2 & 100.2 & NA \\
\hline NC2 & AuNC-BBN-pvp & $1: 1$ & -11.9 & 105.8 & $32.6 \pm 9$ \\
\hline NC3 & $\begin{array}{c}\text { AuNC-BBN-pvp } \\
0.2 \text { mg BBN/mg NC3 (HPLC) }\end{array}$ & $1: 3$ & 25.4 & 111.7 & $5.8 \pm 3$ \\
\hline NC4 & AuNC-PEG & Control & -39.7 & 88.0 & NA \\
\hline NC5 & AuNC-BBN-peg & $1: 1$ & 15.9 & 150.6 & $32.9 \pm 17$ \\
\hline NC6 & $\begin{array}{c}\text { AuNC-BBN-peg } \\
0.13 \text { mg BBN/mg NC6 (HPLC) }\end{array}$ & $1: 3$ & 36.3 & 142.4 & $15.6 \pm 4$
\end{tabular}

Table 4. Physicochemical Properties of Gold Nanoconjugates.

Similarly, there was an increase of $10-40 \mathrm{~nm}$ in hydrodynamic size for BBN constructs indicating conjugation. TEM imaging of heavy-metal uranyl stained samples confirmed the surface coating on the PVP, PEG and Bombesin conjugated nanoparticles (Figure 28). Because these cubic cages were synthesized using silver nanocubes, the inner wall was assumed to be a gold-silver alloy. This formation of this alloyed interior was confirmed by flame atomic absorption spectroscopy (fAAS) that showed a gold-silver ratio of 6:1. Furthermore, to estimate amount of peptide conjugated, nanoconstructs were digested using a cyanide-gold extraction process. In this process, gold atoms in AuNCs react with cyanide $\left(\mathrm{CN}^{-}\right)$to form water-soluble $\mathrm{Au}(\mathrm{CN})^{2-}$ ions ${ }^{43}$. This left the conjugated $\mathrm{BBN}$ to be dispersed in the solution. By measuring BBN's absorbance at $280 \mathrm{~nm}$ and its retention time of 11.9 min using HPLC, we found $1 \mathrm{mg}$ of NC3 to have $\sim 0.2 \mathrm{mg}$ of $\mathrm{BBN}$ on the surface, and $1 \mathrm{mg}$ of NC6 had $0.13-0.15 \mathrm{mg}$ on the surface. 
a

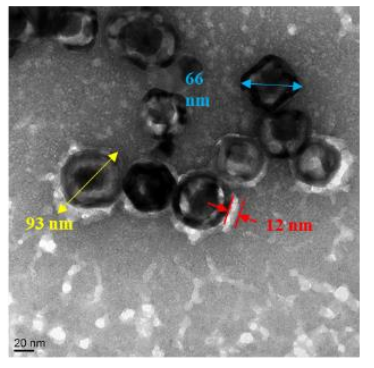

b

C
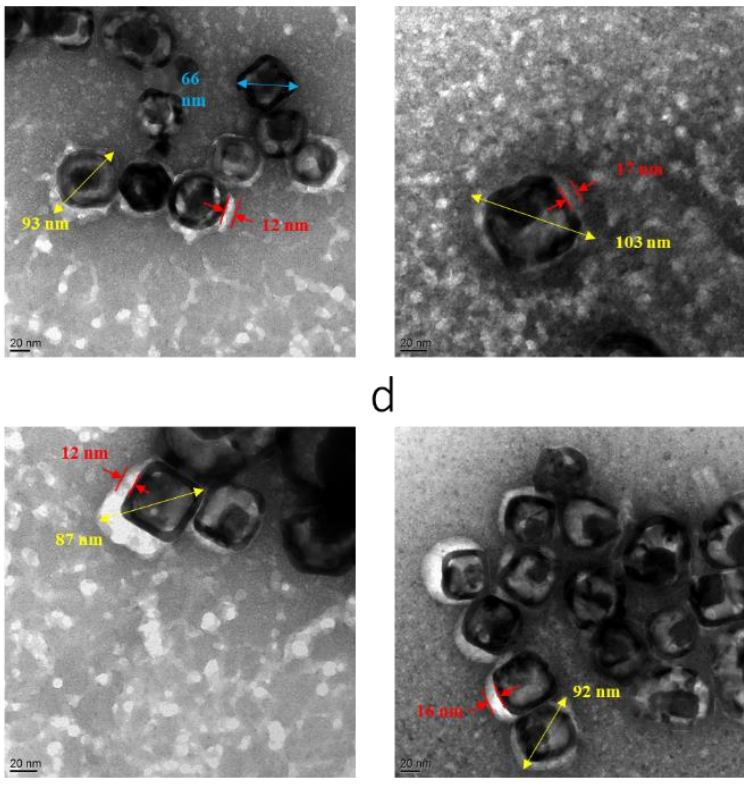

d

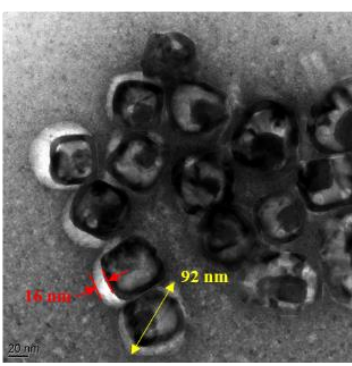

Figure 28. TEM images of nanoconjugates stained with uranyl acetate. (a) NC1; (b) NC3; (c) NC4; (d) NC6. TEM grids were stained immediately after deposition to minimize surface coat contraction due to solvent dissipation. Blue ar row indicates diagonal core length of particles, yellow arrow indicates total hydrodynamic size and red arrow indicates thickness. Results show presence of surface PVP/PEG/Bombesin coating over the nanocages.

\subsubsection{Receptor affinity of BBN conjugated gold nanocages}

BBN-AuNC constructs were treated in vitro with PC3 cells. Because PC3 cells exhibit high GRPR densities ( 44,000 sites/cell) as compared to other cells, the affinity experiments will be sensitive for the BBN constructs ${ }^{44}$. Nevertheless, when constructs were treated with fixed cells, we found high amount of particle attachment to the cell surface only for BBN constructs using dark field fluorescence imaging (Figure 29). The yellow spots in dark field images were confirmed as gold nanocages by matching the hyperspectral profiles for synthesized AuNCs. 

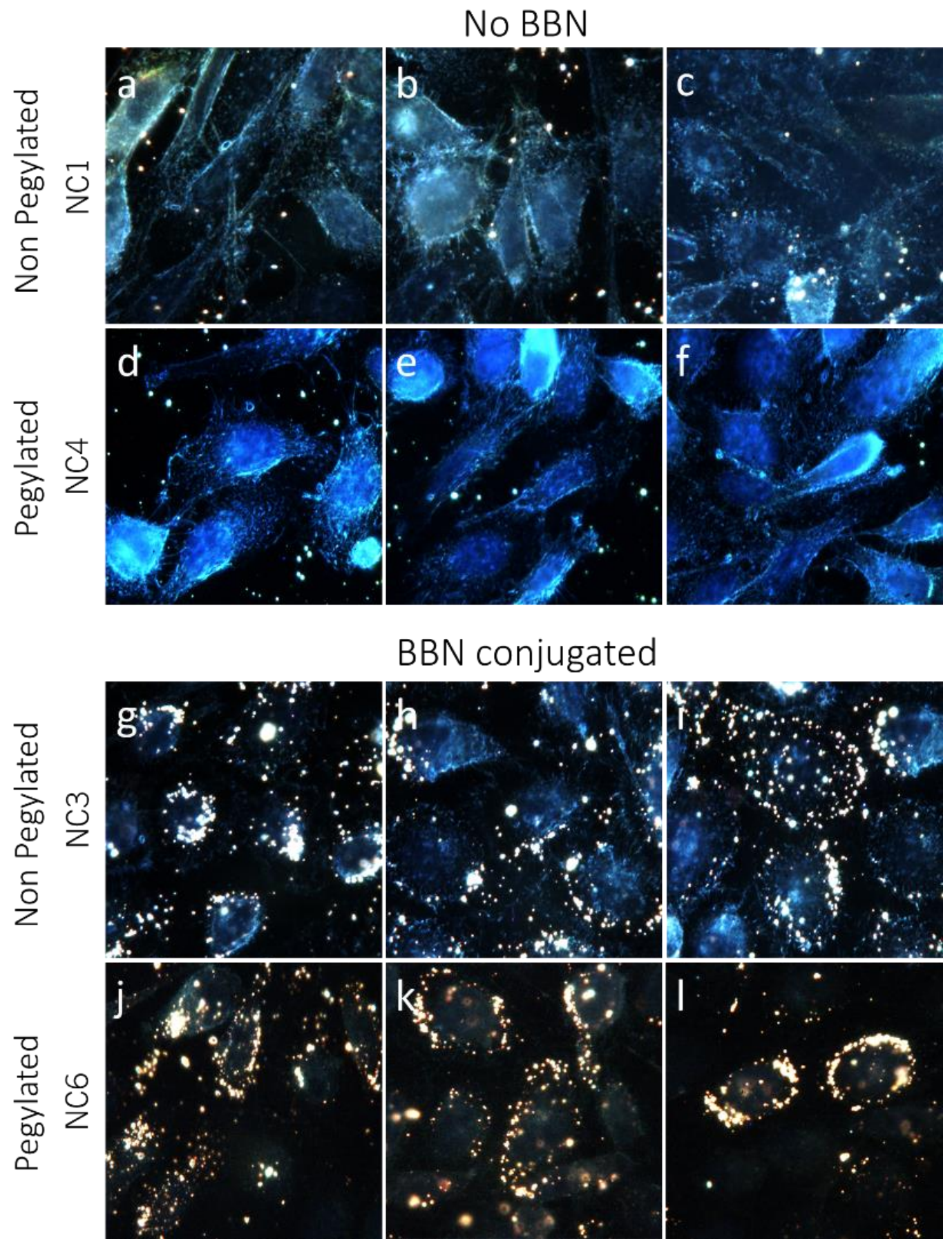

Figure 29. PC3 cells were fixed and treated with constructs. Nanoparticles were allowed to interact and bind with the GRP receptors still present on the cell surface. Bombesin conjugated AuNCs recognize these receptors on the cell surface and bind to them. Representative dark field backscattering images of fixed PC 3 cells after treatment with the following nanoconjugates: $(\mathrm{a}-\mathrm{c}) \mathrm{NC1}$; $(\mathrm{d}-\mathrm{f}) \mathrm{NC} 4$; $(\mathrm{g}-\mathrm{i}) \mathrm{NC} 3$; and $(\mathrm{j}-\mathrm{l}) \mathrm{NC6} .100 \mathrm{X}$ oil. 
As expected, AuNCs without $\mathrm{BBN}$ on surface ( $\mathrm{NC1}$ and $\mathrm{NC4}$ ) did not bind to receptors. These results show that NC3 and NC6 can preferentially bind to GRPR on PC3 cells. We further quantified this affinity using in vitro competitive displacement cell binding assays $\left(\mathrm{IC}_{50}\right)$ on PC 3 cells using radiolabeled ${ }^{125} \mathrm{I}^{-T y r}{ }^{4}-\mathrm{BBN}$. Results show that as the

more $\mathrm{BBN}$ on the surface the more affinity towards PC3 cells. This meant lower $\mathrm{IC}_{50}$ for the nanoconstructs due to higher binding capability. The $\mathrm{IC}_{50}$ values for $\mathrm{NC} 2$, NC3, NC5, and NC6 were $32.6 \pm 9 \mathrm{ng} / \mathrm{mL}, 5.8 \pm 3 \mathrm{ng} / \mathrm{mL}, 32.9 \pm 17 \mathrm{ng} / \mathrm{mL}$, and $15.6 \pm 4$ $\mathrm{ng} / \mathrm{mL}$, respectively. Based on these experiments the BBN-constructs showed very high receptor targeting ability in GRPR expressing prostate cancer cells.

\subsubsection{Mechanism of endocytosis}

Receptor specific pathways such as clathrin and caveolae pathways are dependent on dynamin mediated vesicular budding. Other non-receptor specific pathways include micropinocytosis used for solutes and phagocytosis to engulf large particles. Several studies have shown GRPR utilizes the clathrin mediated endocytosis ${ }^{38,45}$. Based on this data, uniform size and targeting agent, we hypothesized that BBN conjugated AuNC would also utilize clathrin mediated endocytosis. In order to find the mechanism of internalization, each major pathway in the cell was selectively blocked and uptake of nanoconstructs was quantified.

First in order to establish a known endocytic pathway was being used we blocked all modes of pathways using temperature regulation. All known endocytic pathways are energy dependent therefore we studied the uptake of constructs at $4{ }^{\circ} \mathrm{C}$ and compared with regular uptake at $37^{\circ} \mathrm{C}$. Results show little to no internalization in 
cells at $4{ }^{\circ} \mathrm{C}$, confirming a knock energy dependent pathway. Similar results were obtained from Casibang et al, where BBN analogs showed less uptake at $4{ }^{\circ} \mathrm{C}^{46}$. Additionally, results from other cell studies in Hela, bronchial epithelial and adult stem cells, show biomolecule coated gold nanorods did not internalize at $4{ }^{\circ} \mathrm{C}^{47}$. NC6 was then investigated for further mechanistic studies because of its higher stability in physiological media (Figure 14 and 15).

As a means to investigate possible receptor mediation, dynamin dependent pathways were blocked using dynasore hydrate, and cells were treated with NC6. As dynamin ring formation required for vascular scission is arrested after inhibition many of the pit formations at the membrane would cease operations. This will eventually stop endocytosis of clathrin and caveolae mediated pathways. As predicted, results from dark field fluorescence microcopy confirm decreased uptake of AuNC-BBN-PEG in PC3 cells after dynamin ring formation was arrested, while particles internalized in control (Figure 30). This result confirms the participation of dynamin and therefore suggests the use of clathrin or caveolae mediated pathways. Similar results were published by Yang et al. where they showed uptake of nanoclusters using dynamin and clathrin mediated pathways ${ }^{48}$. A previous study by Rotello and coworkers showed that AuNPs with non-targeting cationic ligand entered through dynamin dependent caveolae pathway ${ }^{49}$. However, as our nanoparticles are targeted we posit the use of GRPR internalization by clathrin mediated pathway. Therefore, as a next step we blocked caveolae pathway and studied the uptake in cells. 


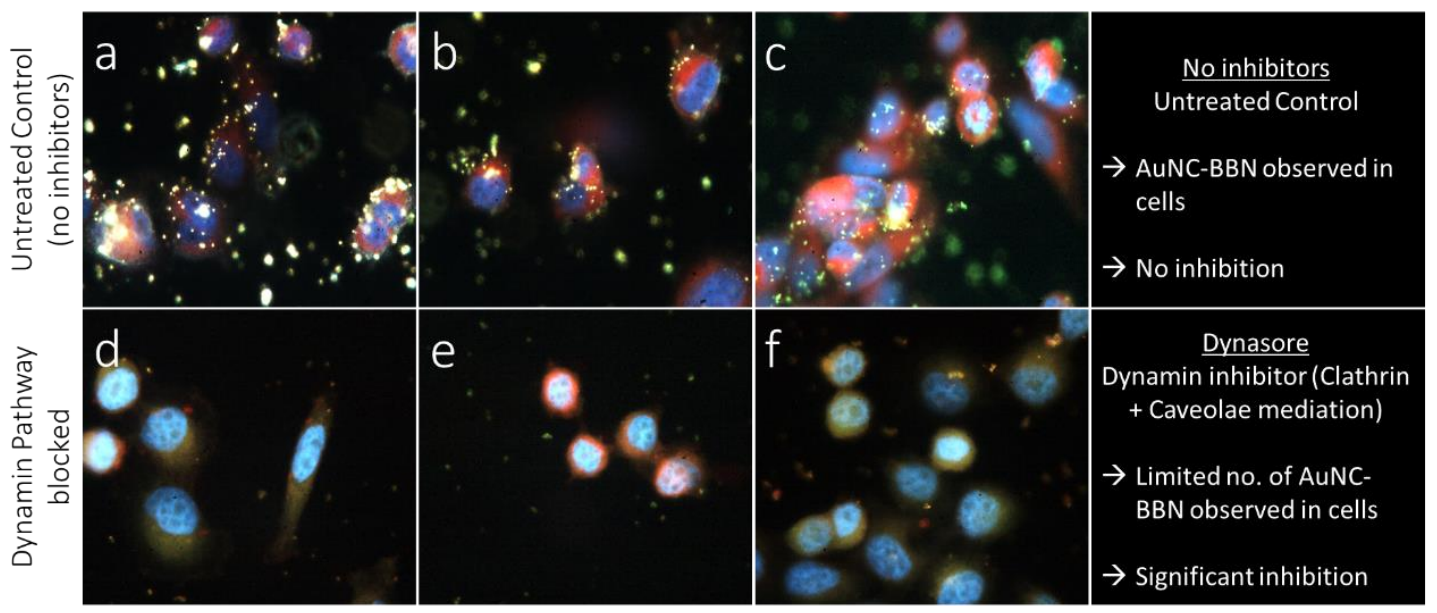

Figure 30. Bombesin conjugated nanocage NC6 requires dynamin for entry in PC3 cells. NC6 was treated with PC 3 cells in the presence and absence of a dynamin inhibitor. Representative dark field images (stained with DAPI and CellMask; $100 x$ oil) of cells in the $(a-c)$ absence and $(d-f)$ presence of dynamin inhibitor.

The caveolae pathway requires formation of pits along the membrane supported by cholesterol dependent caveolin networks ${ }^{50}$. Thus, by removing lipid rafts containing cholesterol the pathway could be inhibited. For this study, we used cholesterol depletors such as methyl- $\beta$-cyclodextrin $(\mathrm{M} \beta \mathrm{CD})$ or filipin on PC3 cells and investigated the uptake of NC6. Results from dark field fluorescence microscopy showed relatively same levels of uptake as control indicating inhibition of caveolae networks did not arrest uptake of AuNC-BBN. This information is in line with GRPR endocytosis using clathrin networks. These results are also similar to inhibition work by Zhao et al. where they proposed the use of clathrin mediation for receptor internalization ${ }^{51}$.

During the clathrin mediated endocytosis, AP2 a recruiter molecule calls for amphiphysin and clathrin molecules to initiate endocytosis ${ }^{52}$. While clathrin forms the pit, amphiphysin recruits dynamin to bud the pit in order to form a vesicle. In this study, we blocked each molecule separately and studied the uptake of NC6 in PC3 cells. Cells were blocked using chlorpromazine (AP-2 blocker), or Pitstop-2 (amphiphysin 
interferer) and X-22 clathrin antibody (clathrin inhibitor). Results from dark field fluorescence microscopy indicated that AuNC-BBN uptake in all 3 separate studies was arrested (Figure 31).

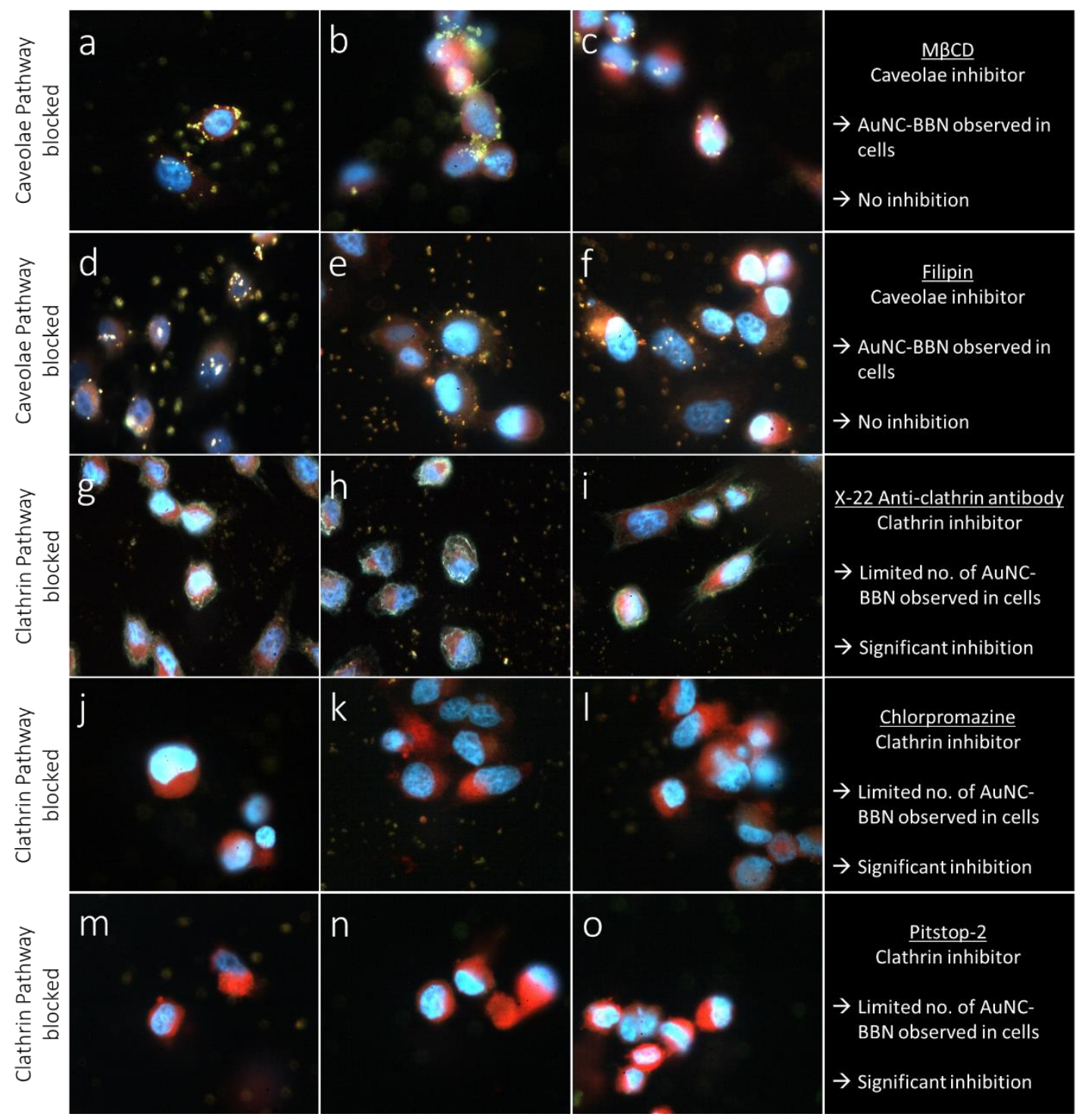

Figure 31. Caveolae inhibitors $M \beta C D$ and filipin did not influence the internalization of NC6 in PC3 Cells, whereas clathrin inhibitors anti-clathrin antibody, chlorpromazine and pitstop-2 significantly influence the internalization. Representative dark field images (stained with DAPI and CellMask; 100x oil) of cells treated with caveolae inhibitors (ac) MBCD; (d-f) Filipin; and clathrin inhibitors (g-i) X-22 anti-clathrin antibody; (j-1) Chlorpromazine; and (m-o) Pitstop2 . 
The results confirm that clathrin mediated endocytosis is responsible for internalization of bombesin conjugated gold nanocages that target the GRPR receptor. We quantified the amount of nanoaggregate/ cell in the images using hyperspectral dark field image analysis. Average number of nanoaggregates in 200 cells were compared with controls and samples blocked with caveolae inhibitors. This analysis revealed a decrease of $85 \%$ for both clathrin inhibitors (chlorpromazine and pitstop-2) and an $81 \%$ decrease using the antibody (Figure 32).

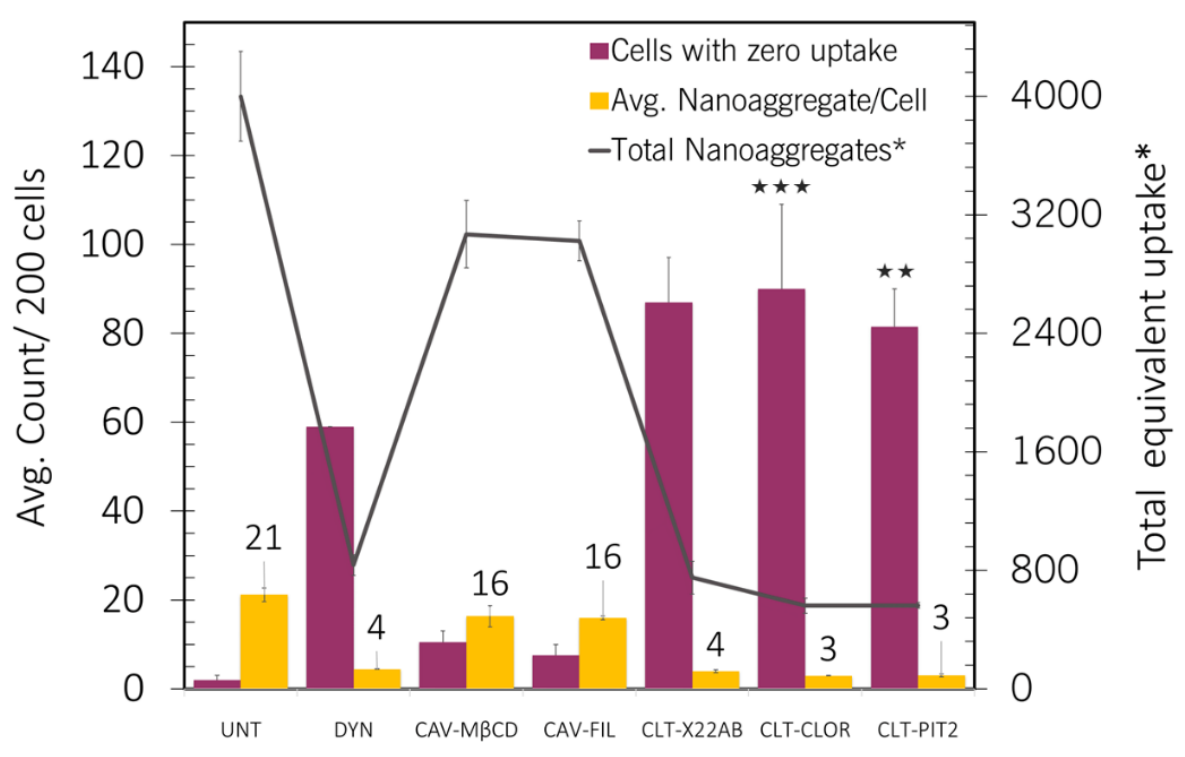

Figure 32. Quantitative analysis of NC6 internalized in PC3 cells in the presence and absence of caveolae and clathrin inhibitors using optical microscopy technique (dark field fluorescence illumination). The legend indicates the following:

(UNT) untreated; (DYN) dynamin pathway blocked with Dynasore; (CAV-M $\beta C D)$ caveolae pathway blocked with methyl- $\beta$-cyclodextrin; (CAV-FIL) caveolae pathway blocked with filipin; (CLT-X22AB) clathrin pathway inhibited during colocalization by anti-clathrin antibody; (CLT-CLOR) clathrin pathway blocked with chlorpromazine; (CLT-PIT2) clathrin pathway blocked by Pitstop-2. The nanoparticles in two hundred cells were monitored and quantified. Each visible gold signal, i.e., nanoaggregate, was approximated as one unit. PC3 cells treated with clathrin inhibitors internalize fewer AuNCs. ${ }^{\star \star} \mathrm{p} \leq 0.01$, and ${ }^{\star \star \star} \mathrm{p} \leq 0.001$ and the values were analyzed using one-way anova.

As expected caveolar inhibition only reduced the uptake by $23 \%$. Furthermore, the cells after treatment were dislodged and the amount of gold was quantified using Neutron 
activation analysis (NAA). Similar to microscopy results, NAA analysis also confirmed a $60 \%$ and $85 \%$ reduction in uptake of AuNC-BBN when clathrin pathways were blocked using antibody and chlorpromazine respectively (Figure 33). Together, these results signify majority of nanoparticles internalize via receptor attachment mediated by clathrin pathway. Based on these results it is quite possible for particles to endocytose via caveolae pathways as very low levels. These internalizations could be due to particle cross-linking and surface charge based agglomerations ${ }^{13}$.

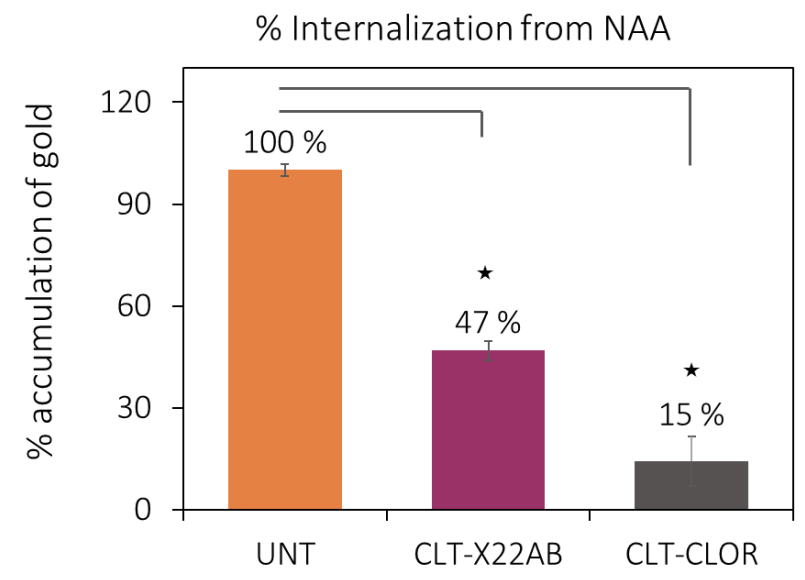

Figure 33. Quantitative analysis of NC6 internalized in PC3 cells in the presence and absence of clathrin inhibitors using NAA. The legend indicates the following: (UNT) untreated; (CLT-X22AB) clathrin pathway blocked with Antibody; (CLT-CLOR) clathrin pathway blocked with chlorpromazine. ${ }^{\star} \mathrm{p} \leq 0.05$, and the values were analyzed using one-way anova.

\subsubsection{Final fate of $\mathrm{BBN}$ conjugated gold nanocages in cells}

In order to understand the intracellular fate of AuNC-BBN in PC3 cells, TEM was used to provide evidence for each step of endocytosis and the fate of nanoparticles. For this step, thin cross sections of treated cells were imaged at multiple locations for observing the plasma membrane interactions, endosomal interactions and cytoplasmic status. We 
observed seven major steps typically seen in clathrin mediated endocytosis, as explained below.

\subsubsection{Step (1) Pre-binding}

As a first step, TEM results showed that majority of AuNC-BBN was present in either monomeric, dimeric or trimeric forms outside the surface (Figure 34). This result proves that particle agglomeration was minimal and it did not exceed the vesicular limit of $300 \mathrm{~nm}$. This information can be interpreted as internalization is likely to take place by clathrin mediation.

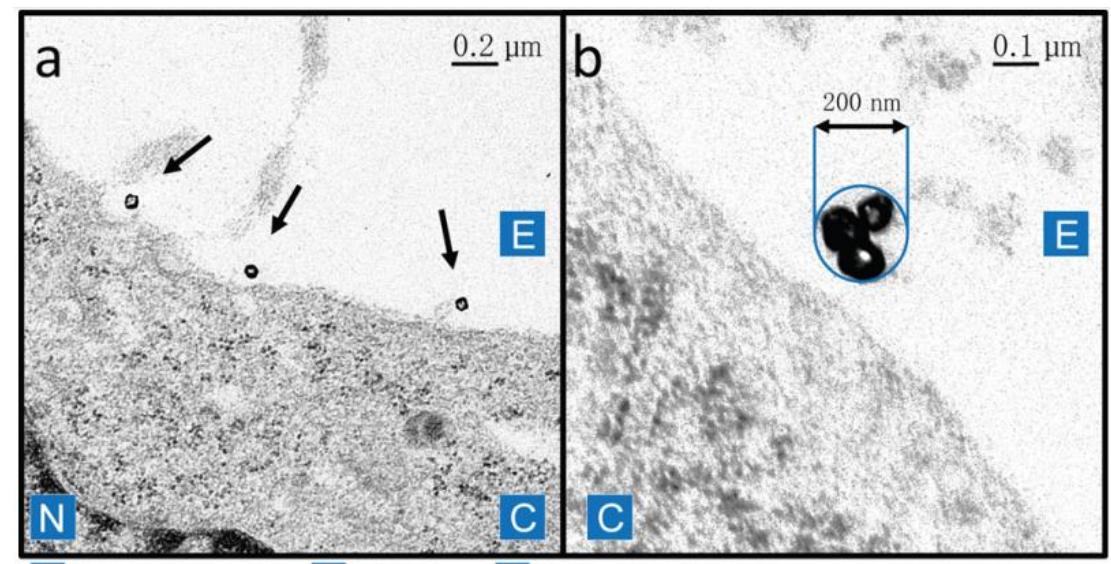

E Extracellular space; C Cytoplasm; $\mathrm{N}$ Nucleus

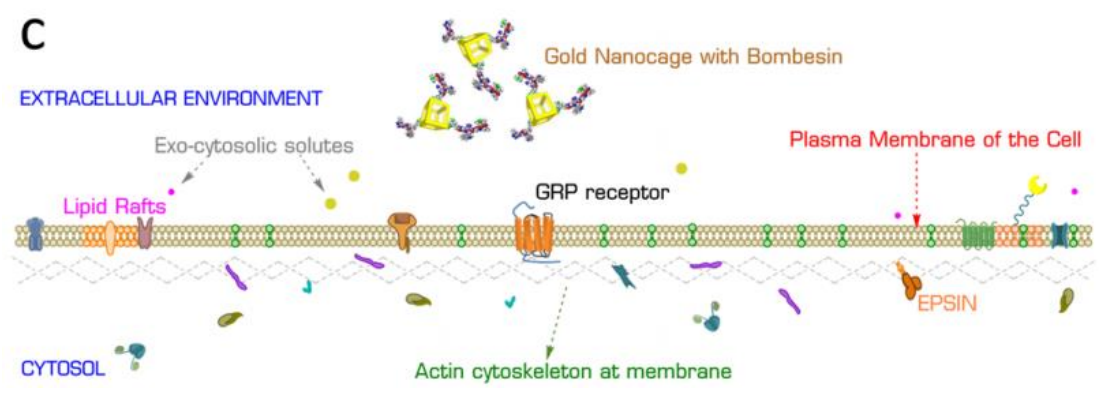

Figure 34. (a, b) TEM images of NC6 in a prebinding cellular environment (see arrows). (c) Schematic representation of the prebinding cellular environment. (Molecules depicted in all subsequent steps can be identified using a glossary available in the Supporting Information; Figure S28.) 


\subsubsection{Step (2) Post-binding signaling cascade and (3) Membrane curving}

TEM images evidently show single or dimeric particle attachment to the cell surface followed by membrane curving and thickening (Figure 35a and 35b). As expected, once bombesin peptide on the nanoparticle surface binds to the GRPR extracellular domain, it would initiate intracellular signaling. This is achieved by phosphorylation of the intracellular $\beta \gamma$-trimeric subunit of the G-protein by G-protein receptor kinase (GRK) ${ }^{53}$. This signal in turn lead to arrestin dependent endocystosis of AuNC-BBN ${ }^{54}$.

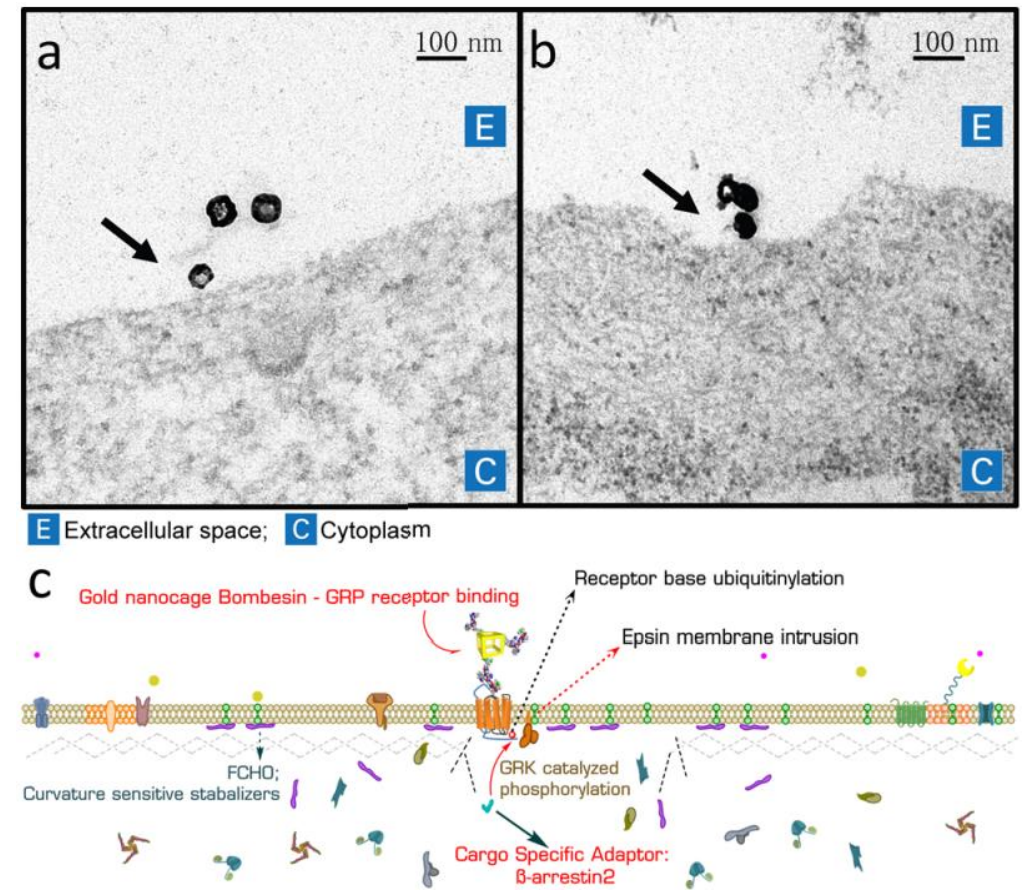

Figure 35a. $(a, b)$ Binding of NC6 with GRP receptors on PC3 cancer cells is shown in TEM images. The TEM images show postbinding signal cascade and membrane intrusion environment within cells (see arrows). (c) Schematic representation of the above environment depicting the important events

Studies have shown this step necessitates Epsin association with Eps15 and intersectin to form the primary invagination complex that allows membrane curving to take place $^{55}$. This complex formation leads to thickening of the membrane for stability and 
allows the AP-2 to recruit clathrin. When cells are blocked with chlorpromazine, AP-2 concentrations are lowered and therefore should not allow formations of clathrin network. Similarly, when X-22 antibody is sent into cells by membrane permeabilization, clathrin molecules are inhibited to form networks within the cell.

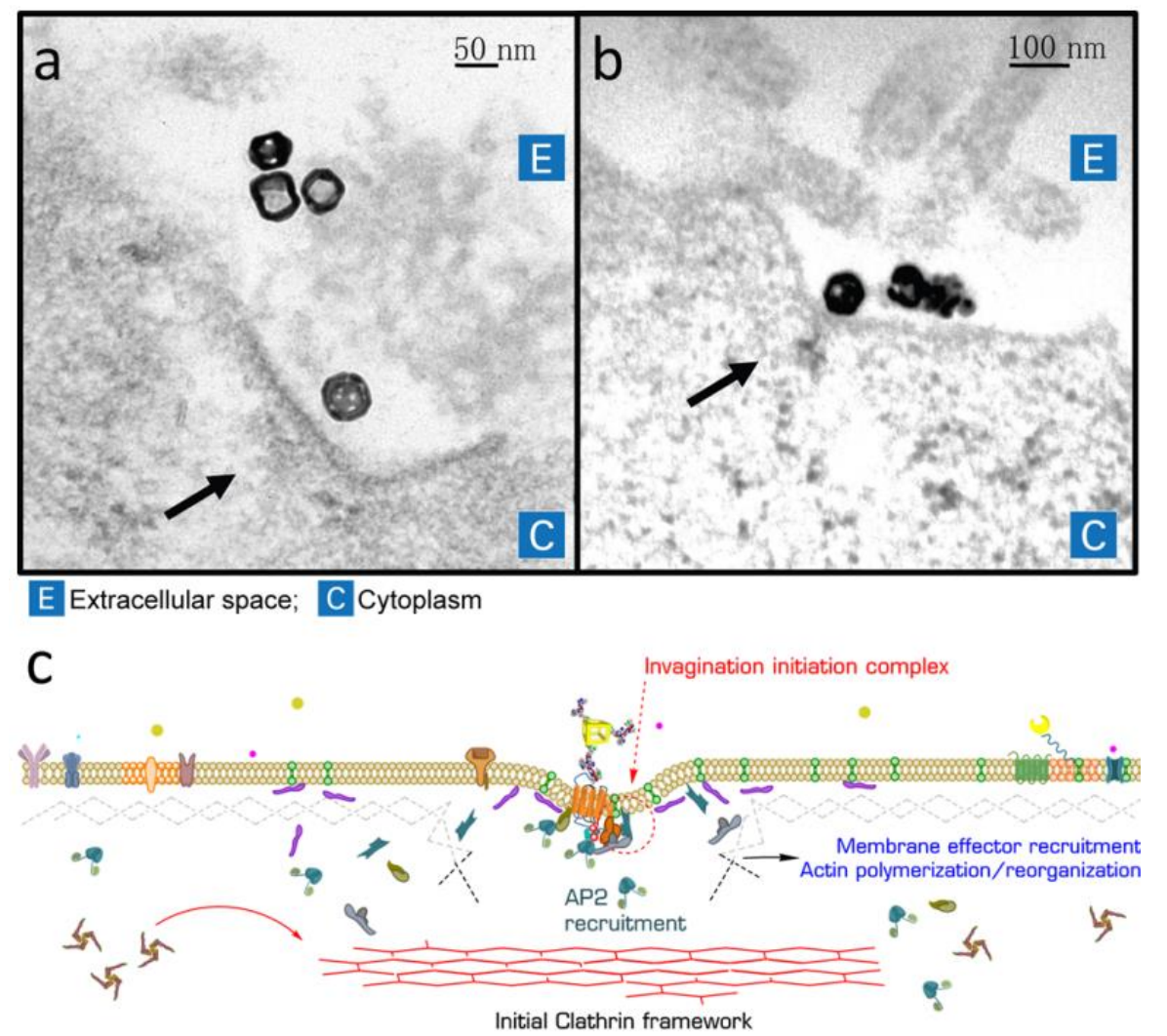

Figure 35b. (a,b) Binding of NC6 results in curving of the cellular membrane as observed in TEM images. Thickening of membrane is observed at these sites (see arrows). (c) Schematic representation of effector recruitment needed for initial curvature.

\subsubsection{Step (4) Clathrin pit formation and (5) Vesicular scission}

TEM images further confirm formation of pits along with membrane that are characteristic signatures of clathrin mediated endocytosis (Figure 36a). In the schemes representing the mechanism, the membrane effectors and scaffolding complexes discussed before, are now 


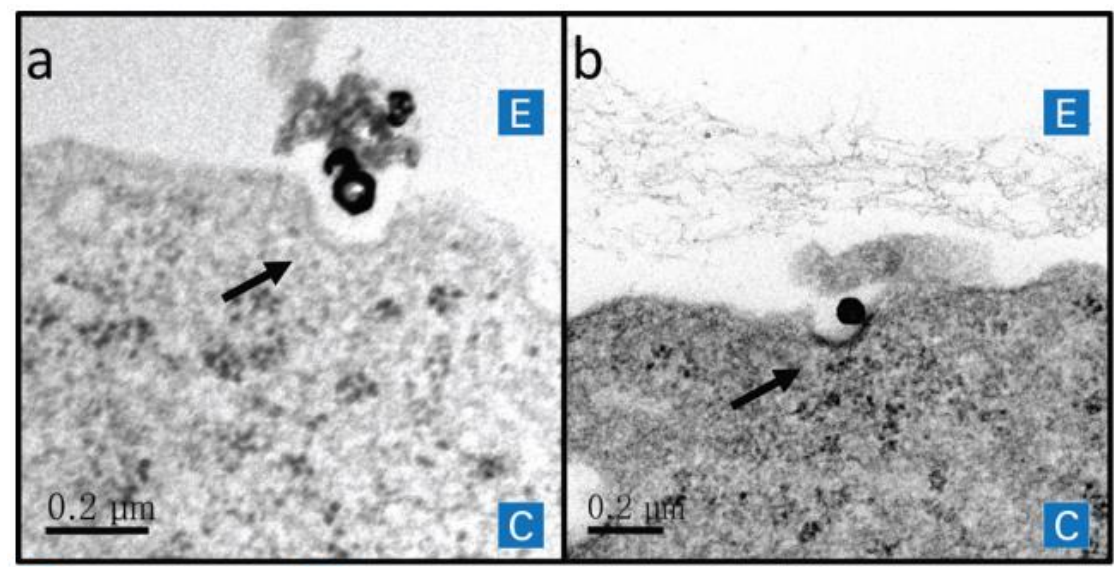

E Extracellular space; C Cytoplasm

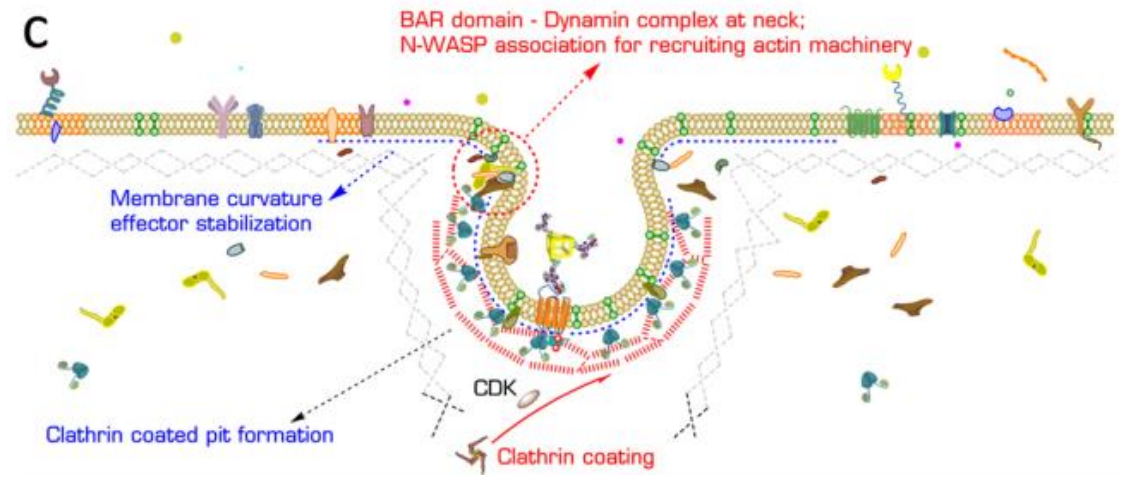

Figure 36a. Formation of clathrin coated pits in the cellular membrane is characteristic of clathrin mediated endocytosis of NC6. $(a, b)$ Before internalization of nanocage the membrane pit formation is observed in TEM images along the cellular membrane (labeled with arrows). (c) Schematic representation of the primary invagination and clathrin cage formation (scaffolding complexes lining the pit are now shown as a blue dotted line for simplicity).

represented as a blue dotted line for simplification sake (Figure 36-38). Further tabulation seen in the images leads to neck narrowing (Figure 36b). Amphiphysin complexes and the dynamin ring is typically formed at the neck for vesicular budding. Interestingly, when the dynamin was inhibited by dynasore, or amphiphysin was blocked by pitstop-2 in our studies, we observed a reduction in uptake. These results show a dynamin dependent uptake of AuNC-BBN in cells. 


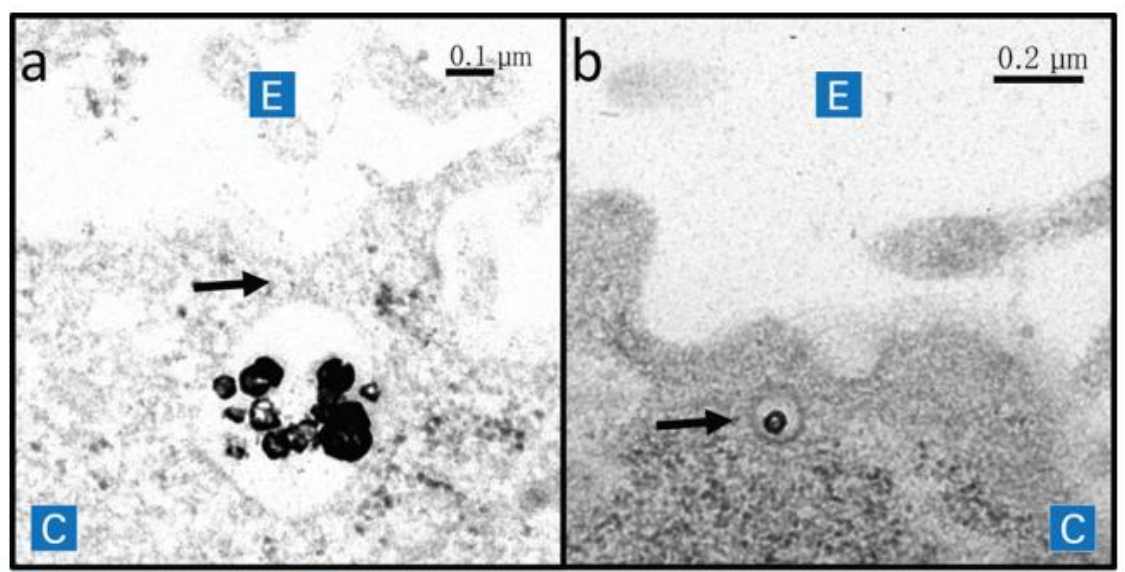

E Extracellular space; C Cytoplasm

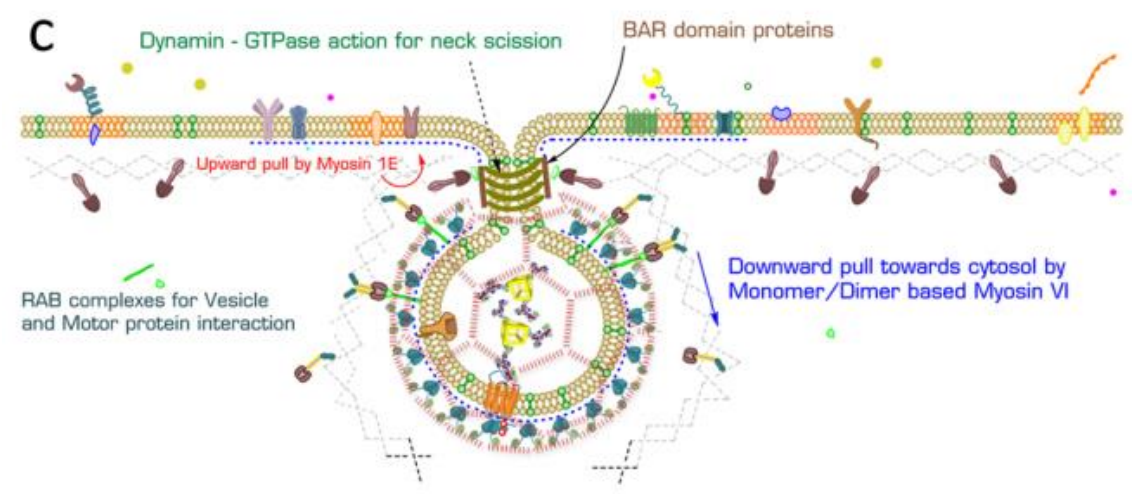

Figure 36b. Transformation of clathrin pits to nascent vesicles. (a,b) TEM images showing pit closure and characteristic clathrin coated vesicles (CCVs). The arrow in part (a) indicates the point of tubulation where the dynamin ring would be present and (b) CCV containing a single nanocage. (c) Schematic representation of scission and budding operations involved in CME.

\subsubsection{Step (6) Endosomal/lysosomal accumulation and (7) Cytoplasmic release}

The final stages as revealed by TEM images proved evidence of cytoplasmic release of AuNC-BBN in PC3 cells. TEM images show vesicles undergoing clathrin uncoating necessary for endosomal transport. In both these vesicles and nascent vesicles being transported, particles were seen attached to the wall displaying the AuNC-BBN-GRPR binding ability (Figure 37). 


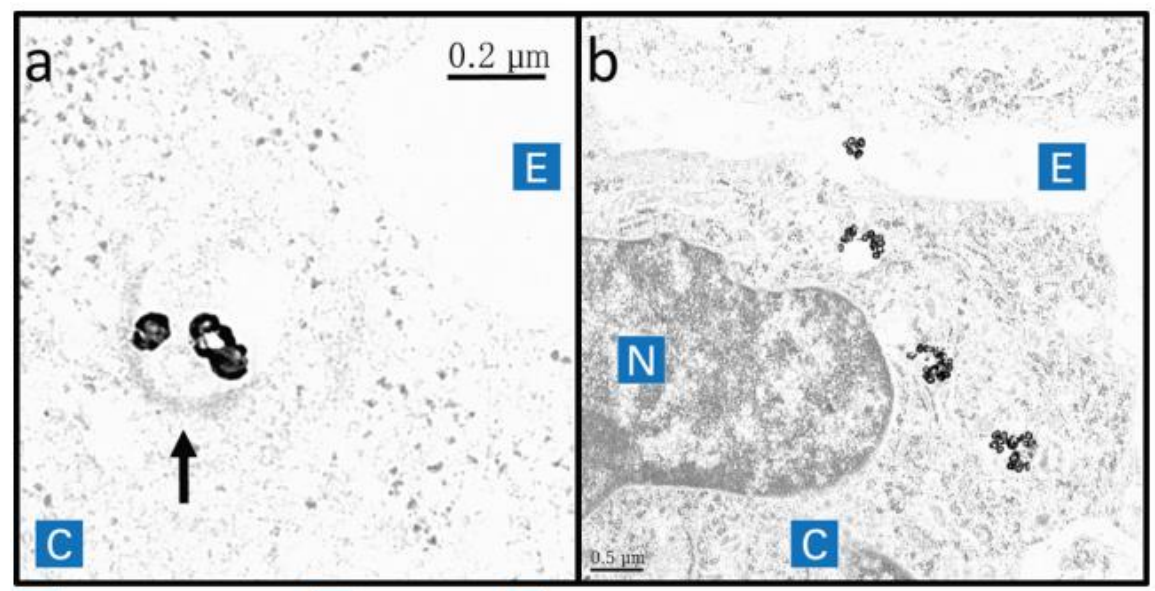

E Extracellular space; C Cytoplasm; $\mathrm{N}$ Nucleus

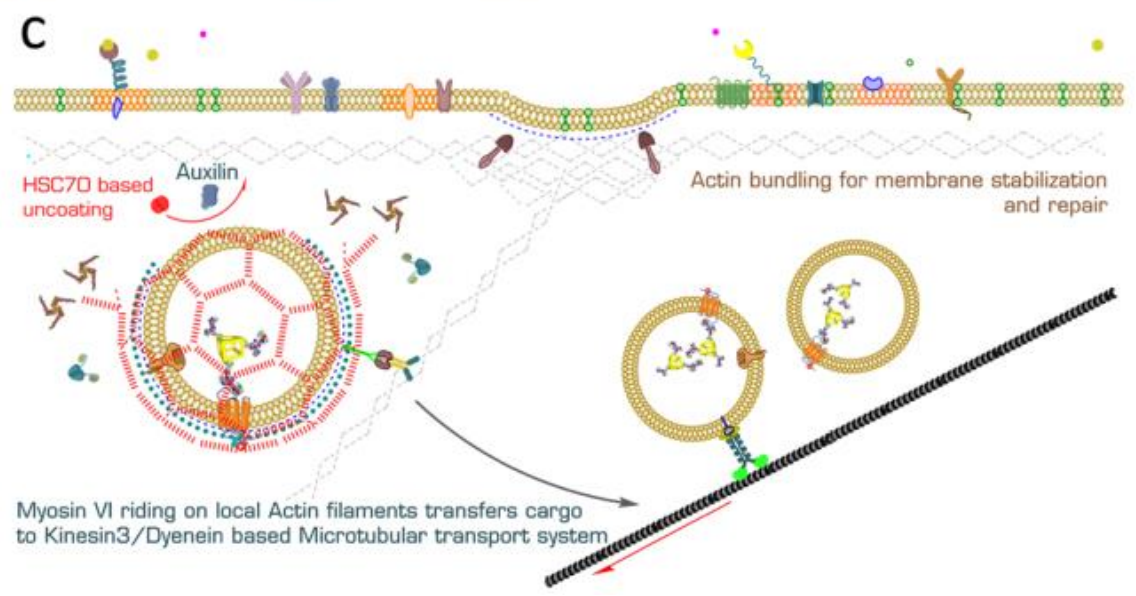

Figure 37. Trafficking of nanocages within cells is controlled by the method of endocytosis. NC6 conjugates were internalized via CME process. (a,b) TEM images show uncoating of clathrin coated vesicles (labeled with arrow) and their transport within cells as early endosomes. (c) Schematic representation of uncoating and vesicular transport of nascent internalized vesicle.

Separately, nanocages were found inside several early endosomes still attached to the wall. However, the amount of nanocages gradually increased in late endosomes or lysosomes present near the nucleus (Figure 38), wherein particles were no longer attached to the inner wall. We expect as $\mathrm{pH}$ steadily drops in late endosomes and lysosomes, particles unbind from the receptor. This has been explained earlier by Le Roy and coworkers as deubiquitination of receptors ${ }^{56}$. Deubiquitination allows cargo 


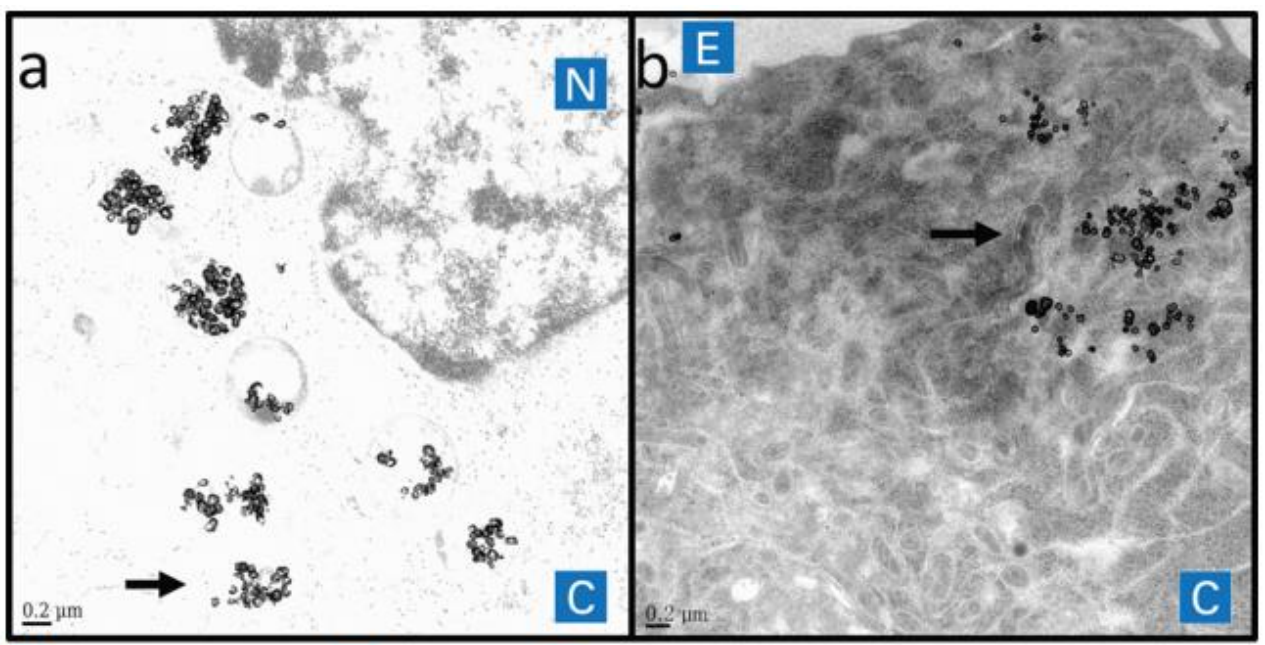

E Extracellular space; C Cytoplasm; N Nucleus

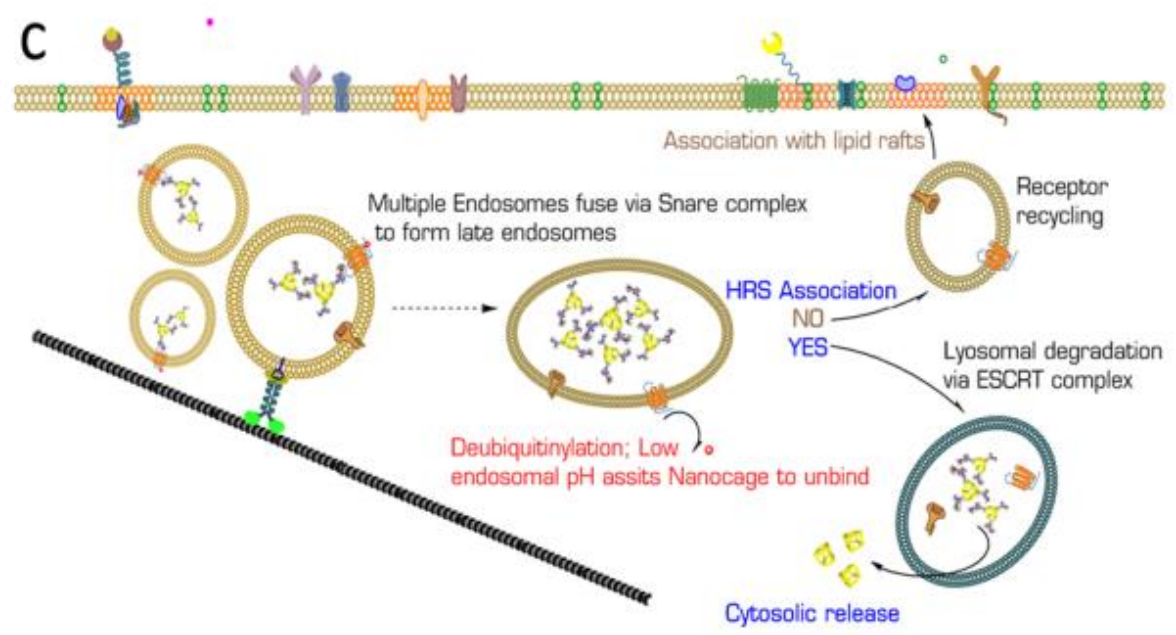

Figure 38. Release of nanocages from lysosome is an important event in trafficking. (a,b) TEM images showing NC6 unbinding within lysosomes. The arrows in the figure indicate AuNCs present in cytoplasm after their eventual lysosomal release. (c) Schematic representation of the endosomal receptor recovery and lys osomal degradation process.

processing components to process the receptor for recycling or degradation based on HRS association factors ${ }^{57}$. But in many cells nanoparticles were seen in the cytoplasm. We expect the large amount of nanocages present in these endosomes and the shape of the cubic particle to play an important role in rupturing the vesicles. Similar results were presented by $\mathrm{Chu}$ et al., where the release of particles from lysosomes to cytoplasm was attributed to anisotropic shape of nanocages ${ }^{58}$. Furthermore, we also investigated particle internalization after inhibition of endocytosis using inhibitors. 
Analysis of TEM images revealed intact cell morphologies and decreased internalization when clathrin mediated pathway was blocked (Figure 39).

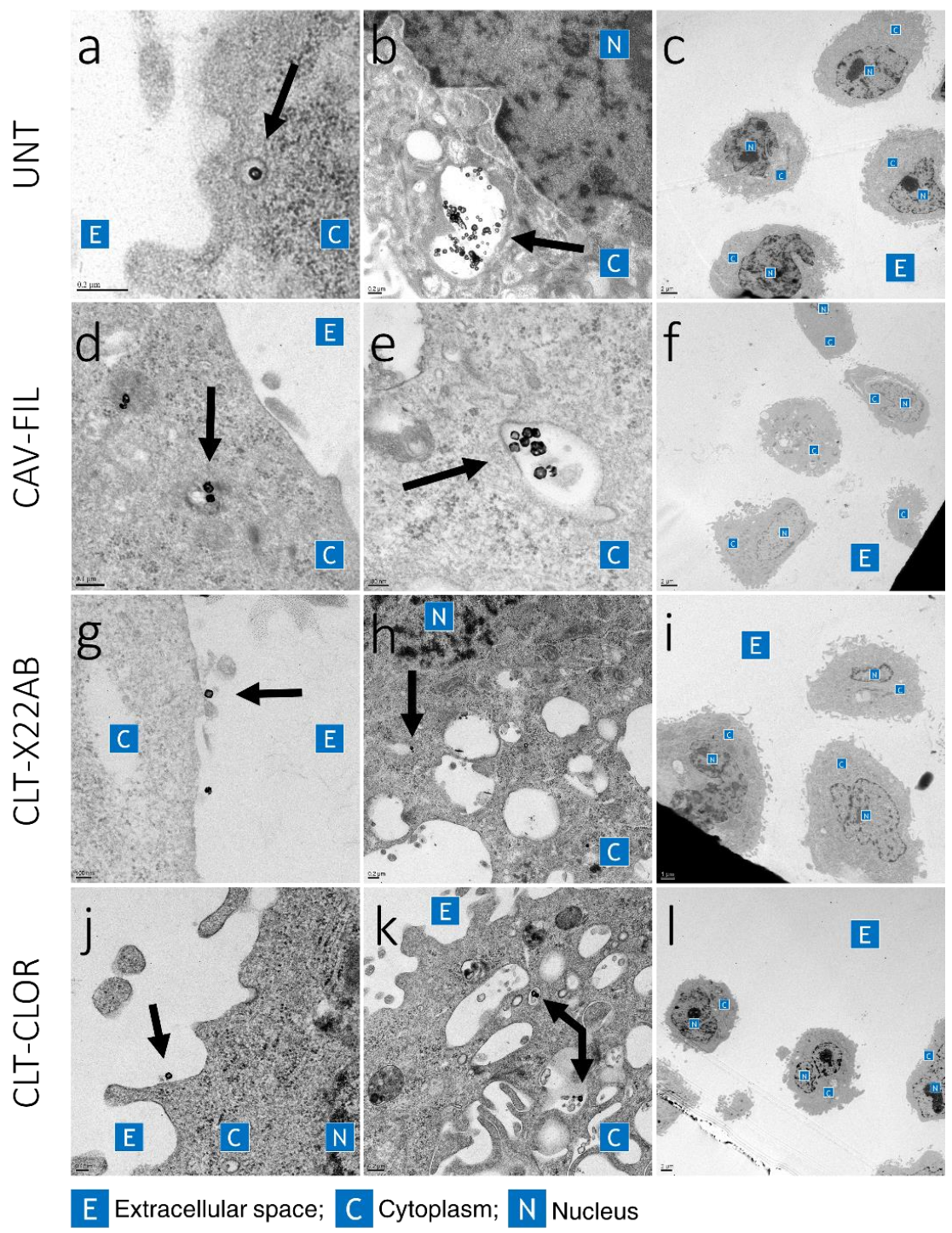

Figure 39. TEM analysis of NC6 internalized in PC3 cells in the presence and absence of caveolae and clathrin inhibitors The legend indicates the follows: (UNT) Untreated; (CAV-FIL) Caveolae pathway blocked with Filipin; (CLT-X22AB) Clathrin pathway inhibited during co-localization by anti-clathrin antibody; (CLT-CLOR) Clathrin pathway blocked with Chlorpromazine. (a, d, g, and j) Representative images for internalization; (b, e, h and k) Representative images for endosomal accumulation; (c, f, i and l) Representative images for cell morphology. Arrows denote nanocages. 
Overall these mechanisms explained in Figures $34-38$, depict an a highly dynamic process with recruitment of several biomolecules including adaptors proteins, recruiter proteins, stabilizer proteins, pit formation proteins and motor proteins working to form a vesicle. These results clearly prove that bombesin peptide still attaches to the GRPR after conjugation to nanocages. In a previous study, Juliano and co-workers also indicated no loss in peptide specificity when conjugated to a passive delivery nanocarrier (AuNCs) ${ }^{45}$. Taken together, peptide functionalized gold nanocages can be used to specifically target cancer cells.

\subsection{CONCLUSION}

Bombesin conjugated gold nanocages have high affinity toward GRPRs that are overexpressed in cancer cells. The high affinity is required for designing targeted therapeutic nanoparticles to achieve high efficacy. This data also confirms that targeting characteristic of bombesin is retained post-conjugation with gold nanocages. Results also provide evidence of GRPR internalizing via the clathrin mediated pathway in cancer cells. Overall, this study validates the important role of surface ligands in internalization and cytoplasmic release of therapeutic nanoparticles for cancer therapy. The comprehensive mechanism established in this study allows for design and development of future targeted delivery of peptide conjugated nanoparticles in oncology. 


\title{
How targeted nanoparticles selectively detect circulating tumor cells (CTC) in blood?
}

\author{
> Enriching CTC using antibody conjugated high affinity nanocubes?
}

\begin{abstract}
Success in cancer therapy requires efficient diagnosis. Currently, tissue diagnostics is performed using an invasive biopsy and further analyzed by immunohistochemistry, FISH, and PCR for staging and treatment planning. However, cancer metastasize by spreading primary tumor cells to distant organs. Therefore, it is possible to isolate circulating tumor cells (CTC) from patients' blood which is easier and less non-invasive than biopsies. The current CTC capture technique relies on EpCAM expression based detection. However, it has been observed metastatic cells which undergo epithelial to mesenchymal transition (EMT) do not express EpCAM and cytokeratin markers on their surface making their detection impossible. Furthermore, with the discovery of tumor heterogeneity and consequent impact on clinical treatment, it is important to detect patients with CTCs early on, based on their genetic alterations which EpCAM based detection lacks. As a solution, a robust magnetic nanoparticle based sensing platform was developed, and Non-small cell lung cancer (NSCLC) was chosen as a viable target. NSCLC is the leading cause for cancer related mortality rates in the US, often associated with $20-35 \%$ response rate and a $\sim 10$ month median survival time warranting a need for effective diagnosis. In NSCLC, both HER-2 (2-5\% mutation incidence) and EGFR (10-35\% mutation incidence) overexpression and their exclusivity in individuals has been seen as a prerequisite in chemotherapeutic selection and dose. Moreover, there is no standardized process to selectively identify CTCs based on HER-2 and EGFR surface expressions. Overall
\end{abstract}


this fact leads us to believe these markers could represent tumor heterogeneity as well. Therefore, targeting these markers may allow us to capture both EpCAM positive and negative cells and also allow EMT characterization.

\section{METHODS}

$20 \mathrm{~nm}$ Magnetic iron nanocubes (FeNC) functionalized with either Herceptin (HER -2 target) or Cetuximab (EGFR target) as markers were developed. FeNC antibody binding capacity was estimated using known Globin protein via ELISA technique. Subsequent separation of spiked live A549 (HER-2 +ve; EGFR +ve) and HCC827 (HER2 -ve; EGFR+ve) cells from a mixture was performed using FeNCs in $1 \mathrm{X}$ PBS, blood plasma and serum to simulate CTC detection. Reduction on non-specific signals and quantification was achieved using fluorescence analysis and counting was based on an automated algorithm. Further EMT status was derived from immunolabelling of isolated live cells.

\section{RESULTS}

Data suggests strong correlation between number of A549 cell captured when Herceptin conjugated MNPs are used (96\% difference vs HCC827), while Cetuximab conjugated MNPs pull both A549 and HCC827. In conclusion, the results show that FeNC based sensing allows both cell marker characterization as well as capture simultaneously. The nanocubes allow better characterization of HER-2 and EGFR positive metastatic cell subpopulations and provide easier prediction of tumor heterogeneity without resorting to invasive procedures. Furthermore, capture efficiency is higher due to size and magnetic moment imparted by its cubic shape. 


\section{CHAPTER 2}

\subsection{INTRODUCTION}

Early diagnosis of cancer in patients can help increase survival chances. Early diagnosis helps medical professionals to evaluate the state of the disease and stop cancer in its tracks. Diagnosis involves analysis of patient history, biological activity and metabolic or disease precursor identification. These precursors if caught early can lead to early prevention, as well as increase drug efficacy and decrease treatment times. However, current state of diagnosis is not often sensitive and diagnosis is usually performed late due to delayed symptoms in patients $^{59}$. On a separate note, though the advances in diagnostic tools have improved the sensitivity in detection capabilities, clinical evaluations often do not correlate well. These factors may include; type of biomarkers to be analyzed in patients or at what stage these might be present in the body for adequate quantification. Recent clinical data has shown each patient may have a distinct biomarker in their system and therefore one cancer biomarker might not represent a population ${ }^{60-62}$. This finding shows that one type of treatment might not be successful in all patients. Thus, not only we need to identify different sets of biomarkers, we need to precisely identify which treatment might benefit specific patients. Furthermore, many of these diagnostic approaches require invasive tests that increase chances of surgical related issues. Therefore, there lies a clinical need for improving diagnostic approach using personalized medicine for cancer treatment.

\subsubsection{Current diagnostic approaches}

Currently, cancer is diagnosed by a multitude of approaches after obtaining tumor tissue from patient. Primarily, patient tissue is obtained via an invasive tumor biopsy. This tissue is 
further investigated for identifying pathological patterns via microscopy. Separately the tissue is either dissected or lysed to identify protein biomarkers indicative of oncogenic activity. Typical methods used for biomarker identifications include immunohistochemistry (IHC), fluorescence in situ hybridization (FISH) and reverse transcriptase polymerase chain reaction $(\text { RT-PCR })^{63}$. IHC identifies heterogeneous or homogenous cell populations based on cell antigens ${ }^{64}$. These antigens can be present both intracellular or as receptors on the membrane. Typically, antibodies/peptides labeled with fluorophores are used to identify the biomarkers. On the other hand, FISH is used to identify genetic abnormalities. In FISH, fluorochrome probes are used to mark telomeric and centromeric ends ${ }^{65}$. In cancerous cells, these fusion breakpoints in the genes are typically disturbed due to gene arrangements by oncogenic mutations. These genetic changes that are identified, explain the protein upregulation within these cells. Although these approaches can identify specific markers, both immunohistochemistry and FISH are qualitative. For a more quantitative approach, RT-PCR or reverse transcriptase polymerase chain reaction is utilized in both single as well as highthroughput assays to identify RNA expression ${ }^{66}$. Similar to FISH, RNA extracted from lysed tissue can give information about gene expression as well as protein upregulation in the cancer cells. However, all these methods rely on tissues obtained from patient via an invasive biopsy. Furthermore, the odds of performing patient biopsy are beyond the timeframe of early disease condition due to delayed symptoms. Also patients during treatment undergo various biochemical changes and genetic mutations that can have significant impact in selecting drugs. These changes are often manifested as acquired drug resistance that worsens disease progression. In these cases, biopsy cannot be used as a means of real time monitoring of patients. Therefore, there is an urgent clinical need to identify biomarkers by non-invasive 
diagnostic procedures using biological fluids such as blood, serum and urine that would help real-time monitoring of patients during treatment. In fact, recent studies on patient blood samples have shown presence of tumor cells and their components ${ }^{67}$. Consequently, these components such as circulating tumor cells (CTCs), cellular exosomes or DNA can be identified in the blood to provide valuable tumor information ${ }^{68}$. Thus, a new approach of detecting CTCs is of considerable importance in novel diagnostics.

\subsubsection{Present detection technologies for Circulating tumor cells}

CTCs are a major subset of the tumor components found in the blood shed by the primary tumor $^{69}$. CTCs are living malignant cells in the circulating blood system that lead to metastases $^{69}$. Metastases promote secondary tumor sites within the body away from the primary tumor leading to disease progression. As CTCs are generated from an invasive tumor region, these cells harbor vast amount of tumor information within them. This information can relate to the protein upregulation and receptor expression or gene expression and mutations. Most of the commonly available detection technologies capture CTCs from the blood using an anti-epithelial cell adhesion marker EpCAM. For example, FDA approved products such as CellSearch $^{\circledR}$ rely on EpCAM coated magnetic beads to collect and enrich CTCs for further analysis. However, EpCAM based devices are approved only for certain types of cancers. For instance, CellSearch ${ }^{\circledR}$ is used for breast, prostate and colorectal cancer while not for lung cancer ${ }^{70}$. Previous reports on lung cancer cases have shown that the CTC data captured using EpCAM beads were poorly correlated in certain patient groups and showed less detection efficiency. Consequently, erroneous data can lead to poor treatment choices and decrease the survival rate. Therefore, it is imperative to develop sensitive techniques that can capture 
different types of CTCs with high efficiency in different patients. For such precision and specificity in capturing CTCs, the detection markers would play a major role. However, there is lack of information to identify these markers due to difference in oncological behavior in different cancers. Thus, it is vital to understand underlying mechanism of CTC generation and their biochemical makeup for us to design new capturing markers.

\subsubsection{Epithelial to mesenchymal transition}

Recent studies have shown that CTCs are generated, when the tumor cells tend to migrate, invade the stroma and shed in to the blood stream ${ }^{71}$. Analysis of these cells revealed a loss of epithelial characteristics and an increase of mesenchymal properties ${ }^{72}$. This change is observed in almost all cancers with non-small cell lung cancer (NSCLC) being most predominant ${ }^{73}$. Studies have shown cancer cells in NSCLC undergo epithelial to mesenchymal transition (EMT) in order to migrate ${ }^{74,75}$. In this process cells lose their epithelial characteristics and therefore suggests loss of EpCAM, a primary marker for capturing CTCs (Figure 40).

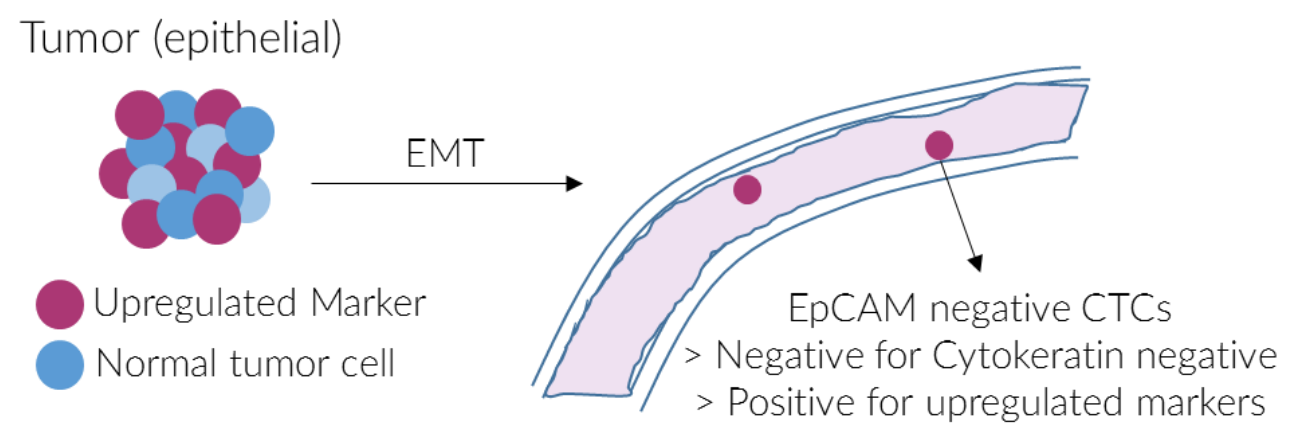

Figure 40. Tumor shedding caused by EMT upregulation in tumor cells. 
The EMT pathway lead to upregulation of certain oncogenes such as Vimentin, Slug/Ras pathway and $\mathrm{N}$-Cadherin ${ }^{76}$. These kinds of proteins allow degradation of the extracellular matrix, increase metalloproteinase activity and in turn give rise to EMT morphology ${ }^{77,78}$. EMT morphology include elasticity of cells required for migration and invasion and ability to quickly proliferate. These properties have also been documented in acquired drug resistance during treatments in NSCLC patients ${ }^{79}, 80$. Overall, these studies suggest that EMT may be the primary pathway for cell proliferation and survival during stress. Previous studies have shown certain tyrosine kinase protein families to be upregulated during this process ${ }^{81-83}$. These proteins are often associated with disease progression and mutation generations ${ }^{84,85}$. Proteins such as EGFR and HER-2 belonging to tyrosine kinase family have been shown to play a crucial role in regulating $\mathrm{EMT}^{86,87}$. In fact, treatment of lung cancer begins with analysis of these markers and several drugs in the market target these receptors ${ }^{88}$. These finding suggest that these pathways (EGFR/HER-2) may be upregulated before and after EMT. Additionally, EGFR and HER-2 expression might actually increase during EMT if not stay same. Based on the reported studies, we hypothesized that these biomarkers can be used to target and detect CTCs irrespective of their EpCAM expression. Furthermore, analyzing CTCs based on EGFR or HER-2 markers levels might also reveal patient information for early diagnosis and drug selection as well as during treatment to avoid acquired resistance. 


\subsubsection{Ultrasensitive detection using nanoparticles}

Current technologies used in isolating CTCs involve the use of large magnetic beads. Limitations of these beads include large size $(>100 \mathrm{~nm})$ and tendency to aggregate. Specifically, magnetic beads are ferromagnetic in nature and have inherent magnetic properties and can result in aggregation. These properties lead to low specificity and magnetic capture ability. To overcome this limitation, we designed $20 \mathrm{~nm}$ magnetic nanocubes with greater magnetic moment and paramagnetic properties compared to the magnetic beads. Therefore, these nanoparticles do not contain residual magnetization in absence of an externally applied magnetic field for capturing them. As they are devoid of inherent magnetization, these particles do not agglomerate and achieve high solvent stability. Additionally, the cubic shape has larger surface to volume ratio, and higher force of attraction per given angle as compared to spherical beads. Anti-EGFR or anti-HER-2 were conjugated on the surface of magnetic nanocubes (MNC) and used for capture. Based on this design parameters, we hypothesize these MNC would be able to selectively target and capture both non-EMT and EMT-CTCs with high efficiency. In this study, we developed techniques to easily capture spiked CTCs from serum using MNC and further characterized their EMT status using protein analysis.

\subsection{METHODS AND CHARACTERIZATION}

\subsubsection{Materials}

Iron (III) acetylacetonate, oleic acid, benzyl ether, poly(maleic anhydride-alt-1octadecene), N-(3-Dimethylaminopropyl)-N'-ethylcarbodiimide hydrochloride (EDC), 
N-Hydroxysulfosuccinimide $\quad$ sodium $\quad$ salt $\quad$ (sulfo-NHS), 2-(Nmorpholino)ethanesulfonic acid (MES), bovine serum albumin, MS-SAFE Protease and Phosphatase Inhibitor, triton X-100, tween-20 and phosphate buffer saline were purchased from Sigma-Aldrich. Hexane, toulene and chloroform were purchased from Acros organics. Sodium hydroxide $(\mathrm{NaOH})$, acetone, sucrose, sodium borate buffer, Hoechst dye, CellMask deep red membrane dye, Pierce ECL Western Blotting Substrate, Pierce BCA Protein Assay Kit and TMB were purchased from ThermoFisher Scientific. B-Actin, Vimentin, EpCAM, Cytokeratin primary antibody kit (7, 8, 17, 18, 19, PAN), EGF, HER-2, both anti-mouse and anti-rabbit secondary HRP-linked IgG antibody were purchased from Cell Signaling. Carboxymethyl-polyethyleneglycolamine was custom synthesized from Nanocs and Laysan Bio. 10x TBS, 10x Tris/Glycine/SDS buffer, 4-15\% 10-well $50 \mu 1$ ready Mini-Protean TGX, Precision Plus Protein Dual color standard ladder and supported nitrocellulose Membrane $(0.2 \mu \mathrm{m})$ were purchased from Biorad. Recombinant Human EGF (carrier-free) and Alexa Fluor 647 anti-Cytokeratin PAN antibody were purchased from BioLegend. Recombinant Human Transforming Growth Factor $\beta-1 /$ TGF $\beta-1$ was purchased from Novoprotein. Amicon $0.5 \mathrm{ml} 100 \mathrm{Kd}$ Regenerated cellulose Ultracel centrifugal filter units were purchased from Merck Millipore Ltd. Cetuximab (Eli Lilly and Company) and Herceptin (Genetech Inc.) were purchased from the hospital pharmacy.

\subsubsection{Instrumentation}

Core nanoparticle size measurements were performed using Joel TEM. Hydrodynamic size and zeta potential was measured using a Malvern Zetasaizer Nano-ZS. 
Centrifugation was performed on a 5424 Eppendorf and refrigerated RC 6+ Sorvall centrifuge. $\mathrm{pH}$ was measured using a SevenCompact Mettler Toledo $\mathrm{pH}$ meter equipped with an InLab Micro electrode. Fluorescence imaging, fluorescence assays and UV-vis absorption assays were performed on a Cytation 5 Cell imaging multi-mode reader. Gel electrophoresis was performed on a Biorad Mini-Protean Tetra system and blots were transferred using a Genscript e-blot transfer system. Western blot imaging and acquisition was performed using Image Lab ver 5.2.1 software on a ChemiDoc XRS system from Biorad. Band densitometry analysis was performed on Image Studio ver 5.2. Solvent extraction was performed on a Büchi Rotovapor R-124 attached to a waterbath and water cooled distillation column. SQUID measurements were performed on a Quantum Design MPMS 3 Magnetometer.

\subsubsection{Synthesis of Iron nanocubes}

Iron nanocubes (FeNC) was synthesized using a high temperature polyol reduction process in organic phase. We designed a novel protocol for synthesizing particles with paramagnetic behavior based on earlier studies ${ }^{89,90}$. Briefly, iron (III) acetylacetonate $(7.06 \mathrm{~g}, 20 \mathrm{mmol})$ was added to a mixture of oleic acid $(1.129 \mathrm{~g}, 12.689 \mathrm{ml})$ and benzyl ether $(104 \mathrm{~g}, 99.71 \mathrm{ml})$ in a 3-neck $200 \mathrm{ml} \mathrm{RBF}$ attached to a reflux condenser and a schlenk line. This solution was degassed using Argon $(20 \mathrm{kPa})$ for $1 \mathrm{~h}$ under constant stirring (400 RPM) and the reaction was kept under argon throughout the process. After initial degassing the reaction temperature was increased to $295{ }^{\circ} \mathrm{C}$ at a heating rate of $20{ }^{\circ} \mathrm{C} /$ min under constant stirring (500 RPM). Reaction color turned jet black from reddish brown after $30 \mathrm{~min}$, and at this stage the solution was cooled down to $100{ }^{\circ} \mathrm{C}$. 
Solution was then quickly cooled on ice and a mixture $(80 \mathrm{ml})$ of hexane, toluene and acetone with a ratio of 1:1:2 was added. This solution was stirred and sonicated to remove particles stuck to the stir bar. The solution was then transferred to a $500 \mathrm{ml}$ beaker with a seal and allowed to precipitate for $15 \mathrm{~h}$. The top solution was decanted and the remaining solution containing precipitate was centrifuged at $20000 \mathrm{~g}$ for $1 \mathrm{~h}$ (15 $\left.{ }^{\circ} \mathrm{C}\right)$. The pellet was then resuspended and washed several times using a mixture hexane and toluene (ratio of 1:1) until a slight clear supernatant formed. For each wash, tube was kept over neodymium magnet (pull force of $250 \mathrm{lbs}$.) for $15 \mathrm{~min}$ followed by slow removal of supernatant using a $10 \mathrm{ml}$ pipette. This solution was then concentrated in hexane and washed 5 times $(15,000 \mathrm{~g}$ for $15 \mathrm{~min})$ until a clear supernatant formed. Finally, the particles (20 mg yield) were resuspended in $4 \mathrm{ml}$ of hexane and stored at room temperature.

\subsubsection{Hydrophilic conversion of Iron nanocubes}

Iron nanocubes synthesized using polyol process resulted in formation of hydrophobic particles soluble only in organic phase. In order to convert them to hydrophilic particles for use in aqueous and physiological solutions, we replaced the organic coating with a functional amphiphilic polymer by modifying a previously published $\operatorname{protocol}^{91}$. Briefly, synthesized FeNCs (in hexane) were injected into a rapidly stirring (500 RPM) Poly(maleic anhydride-alt-1-octadecene) (1.2 g, Avg mol wt. 30,000-50,000) solution dissolved in chloroform $(100 \mathrm{ml})$ in a one neck $200 \mathrm{ml} \mathrm{RBF}$. After $2 \mathrm{~h}$ of stirring, solvent was removed using a rotary evaporator $\left(100 \mathrm{RPM}, 55^{\circ} \mathrm{C}\right)$ attached to standard vacuum line protected by a liquid nitrogen cool trap, until a wet sludge was formed. 
The sludge was quickly mixed with $50 \mathrm{ml}$ of $0.1 \mathrm{M} \mathrm{NaOH}$ solution and dispersed using sonication for $1 \mathrm{~h}$. Solution was then stirred (500 RPM) for $15 \mathrm{~h}$ at room temperature to hydrolyze and open the maleic anhydride rings. The opening of these rings adds free carboxylic groups to the ends of the polymer. These groups can be used to functionalize amines on the nanoparticle surface by $-\mathrm{COOH}$ activation. The solution was washed 2 times with $0.1 \mathrm{M} \mathrm{NaOH}$ solution at $20,000 \mathrm{~g}\left(15^{\circ} \mathrm{C}\right)$ for $1 \mathrm{~h}$, followed by 2 times with 3-layer sucrose density gradient centrifugation $(30 \%, 20 \%$ and $10 \%$ sucrose). Pellet was resuspended and concentrated in $4 \mathrm{ml} 10 \mathrm{mM}$ MES buffer and washed once. As a next step $100 \mathrm{kDa}$ centrifugal filters were equilibrated with MES buffer and used for removing excess polymer (6000g for $6 \mathrm{~min})$. Solution was then again subject to 2-layer sucrose density gradient centrifugation (30\% and $10 \%$ sucrose) at $15000 \mathrm{~g}$ for $15 \mathrm{~min}$, followed by washes with DI water. FeNC pellet was then redispersed in $5 \mathrm{ml}$ water and stored at room temperature.

\subsubsection{Synthesis of pegylated nanocubes}

For added stability and solubility in aqueous solutions, the $-\mathrm{COOH}$ groups of hydrophilic particles were activated using EDC/sulfo-NHS and coated with aminePEG. Briefly, particles were dissolved in 0.1 M MES buffer ( $\mathrm{pH} 4.5$ ) containing $2 \mathrm{mg}$ EDC and $2 \mathrm{mg}$ sulfo-NHS and kept for shaking (850 RPM) for $1 \mathrm{~h}$ at $28^{\circ} \mathrm{C}$. Particles were centrifuged (15000g for $15 \mathrm{~min}$ ) and pellet was dispersed in $50 \mathrm{mM}$ sodium borate buffer containing $10 \mathrm{mg}$ carboxymethyl-polyethyleneglycol-amine $\left(\mathrm{CH}_{3} \mathrm{O}-\mathrm{PEG}-\mathrm{NH}_{2}\right.$, MW 2000) and $5 \mathrm{mg}$ EDC. This solution was kept for shaking (850 RPM) for $15 \mathrm{~h}$ at

$22{ }^{\circ} \mathrm{C}$. After reaction, pegylated FeNCs were washed with water 2 times $(15000 \mathrm{~g}$ for 
15 min), resuspended in DI water and stored at room temperature. Pegylated FeNCs were used for conjugation with antibodies.

\subsubsection{Antibody conjugation}

Hydrophillic pegylated FeNCs were conjugated to antibody via carbodiimide crosslinking using EDC/sulfo-NHS reaction. Briefly, $100 \mu 1\left(2 \times 10^{6}\right.$ particles/ml $)$ of FeNCs were washed with $0.1 \mathrm{M}$ MES buffer ( $\mathrm{pH} 4.5$ ) prior to being activated. The pellet was redispersed in $400 \mu 10.1$ M MES buffer (pH 4.5) with $3 \mathrm{mg}$ EDC and $4 \mathrm{mg}$ sulfo-NHS at $28{ }^{\circ} \mathrm{C}$ for 3.5 hours (shaking at $800 \mathrm{RPM}$ ). After activation, particles were centrifuged (15000g for $15 \mathrm{~min}$ ), redispersed in $100 \mu \mathrm{l} 1 \mathrm{X}$ PBS and mixed with antibody solution (4 $\mu 1$ of Globin $\mathrm{Ab}(10 \mathrm{mg} / \mathrm{ml})$ or $200 \mu 1$ of Cetuximab $(2 \mathrm{mg} / \mathrm{ml})$ or $200 \mu \mathrm{l}$ of Herceptin $(2 \mathrm{mg} / \mathrm{ml})$ ). Reaction was kept in shaker (800 RPM) for $15 \mathrm{~h}$ at 22 ${ }^{\circ} \mathrm{C}$, followed by 2 washings with $1 \mathrm{X}$ PBS (15000g for $15 \mathrm{~min}$ ). Final solution was redispersed in $1 \mathrm{ml} 1 \mathrm{X}$ PBS and stored at $4{ }^{\circ} \mathrm{C}$. FeNC-Globin $\mathrm{Ab}$ was used for estimating protein conjugation efficiency using known globin protein (ELISA), while FeNC conjugated to Cetuximab and Herceptin were used to target EGFR and HER-2 in cell studies. These particles were labeled as FeNC-EGF and FeNC-HER.

\subsubsection{Characterization of nanoconjugates}

FeNC constructs were characterized using TEM and DLS. For TEM, $8 \mu 1$ of $1 / 10^{\text {th }}$ diluted FeNCs were placed over a CF300-Cu carbon film based copper grid and air dried for $10 \mathrm{~min}$ in a hot air oven $\left(40{ }^{\circ} \mathrm{C}\right)$. TEM imaging for polymer coated and 
pegylated FeNCs showed a uniform size of $20 \mathrm{~nm}$ (Figure 41). For DLS measurements, $1 \mathrm{ml}$ of $1 / 10^{\text {th }}$ diluted FeNC solution was placed in a disposable UV-grade cuvette and analyzed. DLS showed that the hydrodynamic size of hydrophilic polymer coated FeNCs and pegylated FeNCs were around $\sim 140 \mathrm{~nm}$, and an increase upon conjugation

a

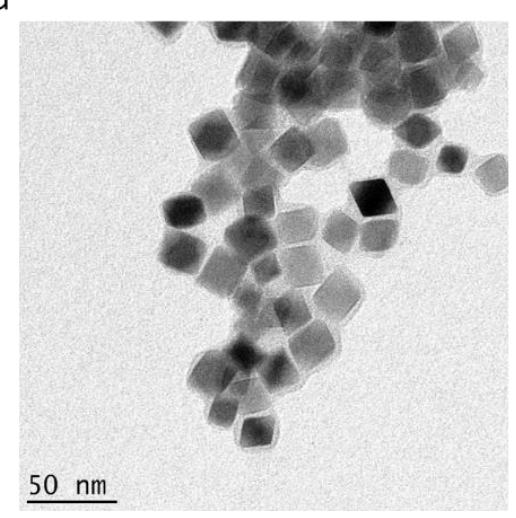

b

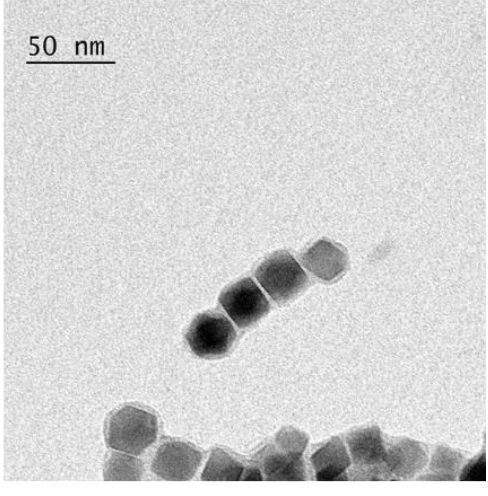

Figure 41. TEM imaging for (a) FeNC coated with polymer and (b) FeNC after pegylation. The cubic particles were uniform with an edge length of $20 \mathrm{~nm}$.

with antibodies $(\sim 170 \mathrm{~nm})$ (Table 5). Zeta potential of polymer coated FeNCs was -63 $\mathrm{mV}$ whereas upon coating and conjugation with PEG and antibody the zeta potential changed drastically to $-6 \mathrm{mV}$. No change in zeta was observed when FeNC - PEG was conjugated with antibody. The magnetic properties were further investigated using SQUID analysis. For this analysis, $5 \mathrm{mg}$ of lyophilized FeNC power was loaded in sealed SQUID sample holders. The data was recorded using the temperature sweeping mode under zero-field-cooled (ZFC) conditions from 2 to $300 \mathrm{~K}$ at $1000 \mathrm{Oe}$. The corrected magnetic moment was then determined by a previously published procedure $^{92}$. 


\begin{tabular}{llll}
\hline Construct & Hydrodynamic Size $($ d.nm) & Zeta $(\mathrm{mV})$ & TEM edge length $(\mathrm{nm})$ \\
\hline FeNC with (polymer) & 148 & -63 & 20 \\
\hline FeNC-Peg & 142 & -5.8 & 20 \\
\hline FeNC-Peg-HER & 171 & -6.5 & 20 \\
\hline FeNC-Peg-CTX & 175 & -5.8 & 20 \\
\hline
\end{tabular}

Table 5. Properties of FeNC constructs synthesized for the study.

\subsubsection{ELISA}

Indirect enzyme-linked immunosorbent assay (ELISA) was used for measuring conjugation efficiency of antibody bound to FeNC. For this study, FeNC-Globin conjugate was used and anti-globin antibody was used as a surrogate. Briefly, $100 \mu 1$ of $0.1 \mathrm{mg} / \mathrm{ml}$ Globin solution was added to wells of flat bottom sterile 96 well plate, and incubated for $15 \mathrm{~h}$ at $4{ }^{\circ} \mathrm{C}$. The wells were then washed with wash buffer $(0.5 \%$ Tween20) followed by blocking with $200 \mu 1$ of $2 \%$ BSA solution for $30 \mathrm{~min}$ at room temperature. As a next step, the wells were washed with wash buffer prior to addition of $100 \mu 1$ FeNC-Globin Ab constructs. FeNC- PEG was used as negative control was used as a negative control while antibody solution was used as a positive control for the experiment. PBS was considered as the blank. The nanoparticle solutions were incubated for $2 \mathrm{~h}$ at $37{ }^{\circ} \mathrm{C}$ followed by washing with wash buffer. $50 \mu 1 \mathrm{of} 0.32 \mu \mathrm{g} / \mathrm{ml}$ secondary antibody (goat anti Rabbit IgG-HRP) was then added to the wells and incubated for $30 \mathrm{~min}$ at $37^{\circ} \mathrm{C}$ followed by 2 washes with wash buffer. Finally, $50 \mathrm{ul}$ of the substrate solution (TMB peroxidase) was added to the wells and the reaction was 
stopped by adding $0.1 \mathrm{M} \mathrm{HCl}$ solution. The plates were read by a Cytation 5 absorbance plate reader at $450 \mathrm{~nm}$.

\subsubsection{SQUID magnetic analysis}

Magnetic iron nanocubes were characterized using superconducting quantum interference device (SQID) equipped with a $7 \mathrm{~T}$ magnet. In this process, Josephson junctions are used to measure a change in magnetic flux using a coil with a known inductance. For the magnetometry analysis, $5 \mathrm{mg}$ of pure lyophilized FeNCs were loaded in a VSM sample holder. Measurements were made using a five quadrant magnetic moment with varying external magnetic field analysis $\left(\mathrm{H}_{\max }\right.$ of $5 \mathrm{~T}$ at $\mathrm{T}=2$ K). Zero-field cooled magnetic susceptibility and field-cooled warming measurements were performed using a sweep mode from 2 to $300 \mathrm{~K}$ at 1000 Oe (Figure 43 and 42). Using this analysis, the blocking temperature was estimated. Raw data was then corrected for sample shape and radial offsets and presented. 


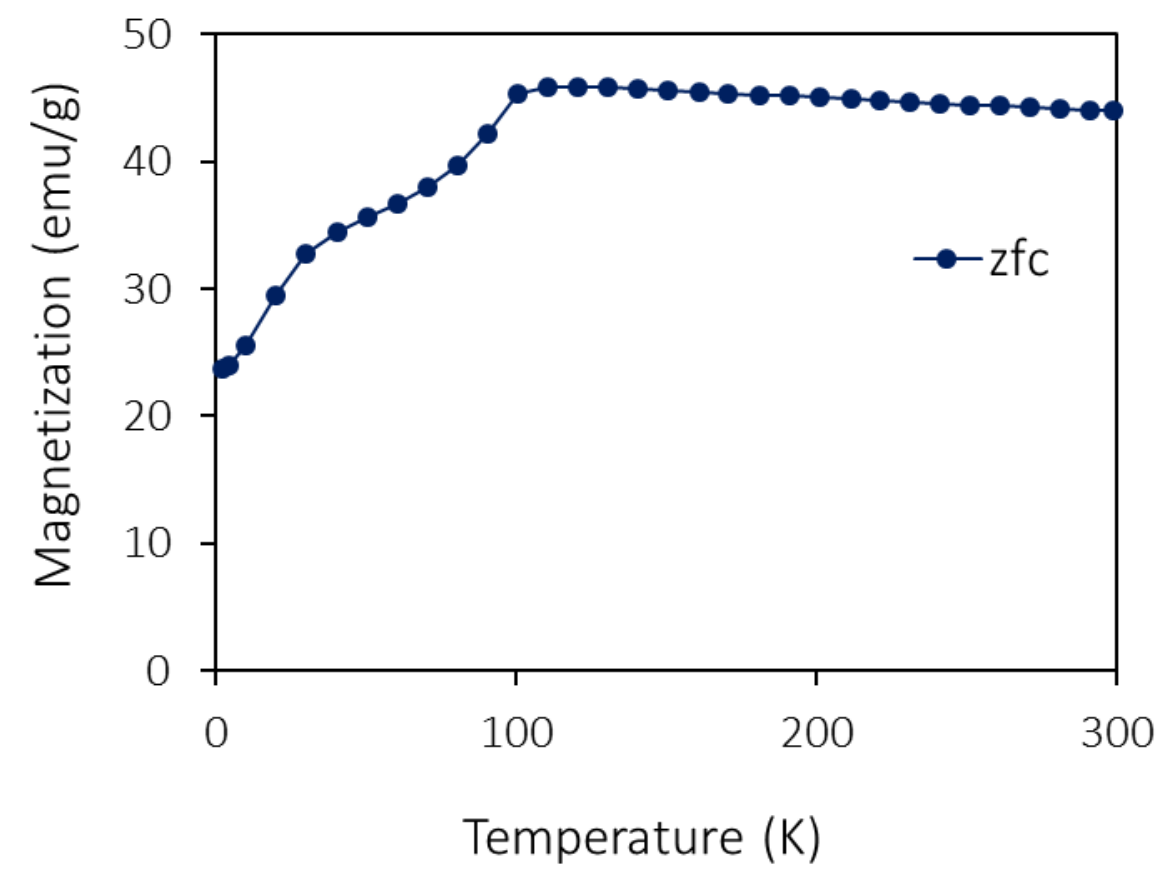

Figure 42. Magnetization vs temperature curve for FeNCs measured at zero field cooled state at 1000 Oe

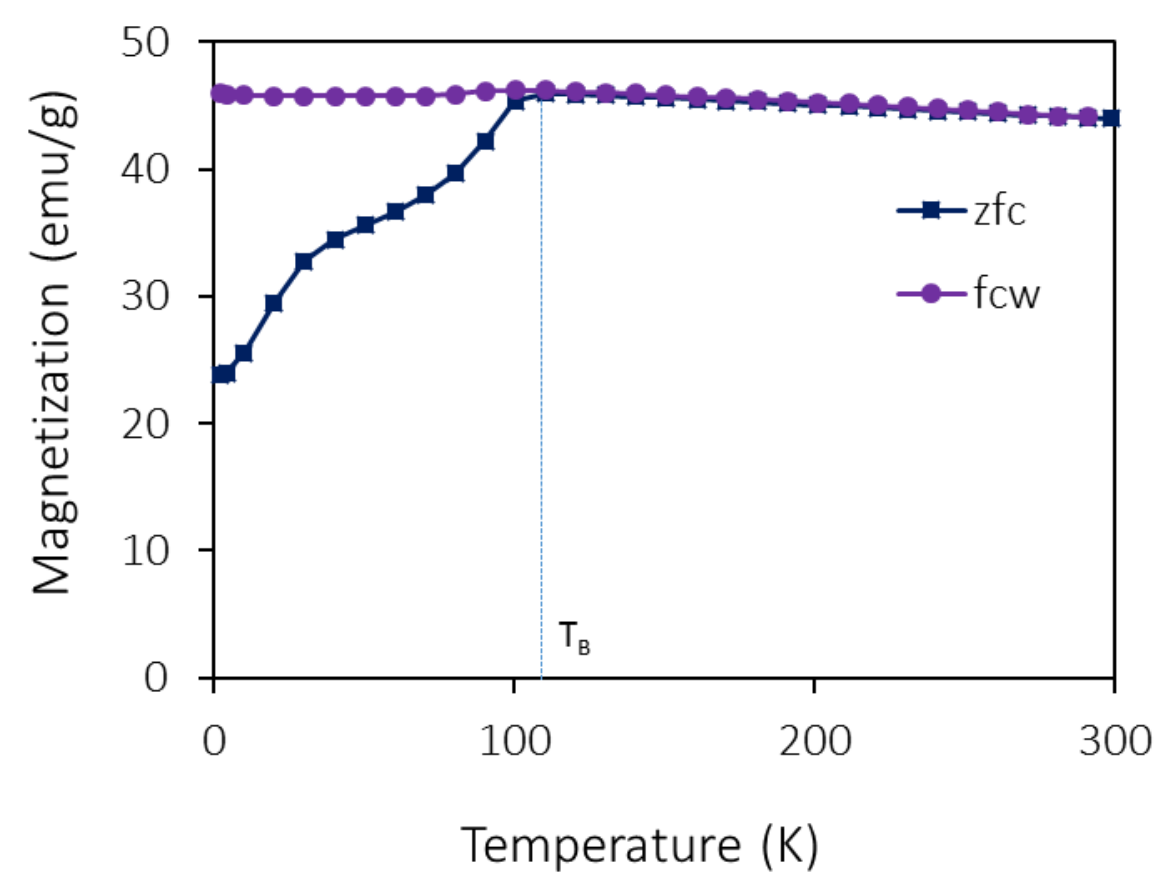

Figure 43. Overlay of magnetization vs temperature curve for FeNCs measured at zero field cooled state and field cooled warm state at 1000 Oe. The blocking temperature was calculated to be $-163.15^{\circ} \mathrm{C}$. 


\subsubsection{Isolation of blood serum}

Pig serum was provided by an investigator at MU for the spiking studies. $5 \mathrm{ml}$ of blood was collected from pigs in a sealed test tube. Clotted blood was centrifuged (100g for 10 min at $4{ }^{\circ} \mathrm{C}$ ) and serum was isolated and stored at $4{ }^{\circ} \mathrm{C}$. CTCs were spiked in freshly derived serum.

\subsubsection{Cell culture}

HCC827 and A549 human non-small cell lung cancer cell lines (ATCC, USA) were grown in RPMI 1640 medium (obtained from Gibco BRL). The media was supplemented with $4.5 \mathrm{~g} / \mathrm{L}$ d-glucose, $25 \mathrm{mM}$ Hepes, $0.11 \mathrm{~g} / \mathrm{L}$ sodium pyruvate, $1.5 \mathrm{~g} / \mathrm{L}$ sodium bicarbonate, $2 \mathrm{mM}$ l-glutamine, 10\% heat-inactivated fetal bovine serum (Atlanta Biologicals, USA), and $1 \%$ penicillin/streptomycin antibiotic solution. Cells were cultured in a humidified atmosphere of $95 \%$ air and $5 \% \mathrm{CO} 2$ at $37{ }^{\circ} \mathrm{C}$ (Thermo Scientific, USA).

\subsubsection{Capture methodology}

For the capture process and detection of spiked CTCs an easy and robust magnetic extraction process was designed (Scheme 2). NSCLC cells with or without EMT treatment were dislodged from the T25 flask at $70 \%$ confluence. Cells not specific to antibody were used as control. For all experiments, target cells (A549/HCC827) were live stained red and control cells (HCC827/MCF-7) live stained blue. Briefly, cells were suspended in 1X PBS and incubated with $40 \mu \mathrm{l}$ of Hoechst (blue) dye or $40 \mu \mathrm{l}$ of CellMask Deep red stain for $20 \mathrm{~min}$ at $37{ }^{\circ} \mathrm{C}$. As a next step, capture experiments relied on live cells, while 
cytokeratin detection was performed on fixed cells. The cells were washed with 1X PBS twice to remove excess dye (2000 RPM for $5 \mathrm{~min}$ ). Cells were counted and cell stock solution was prepared (known

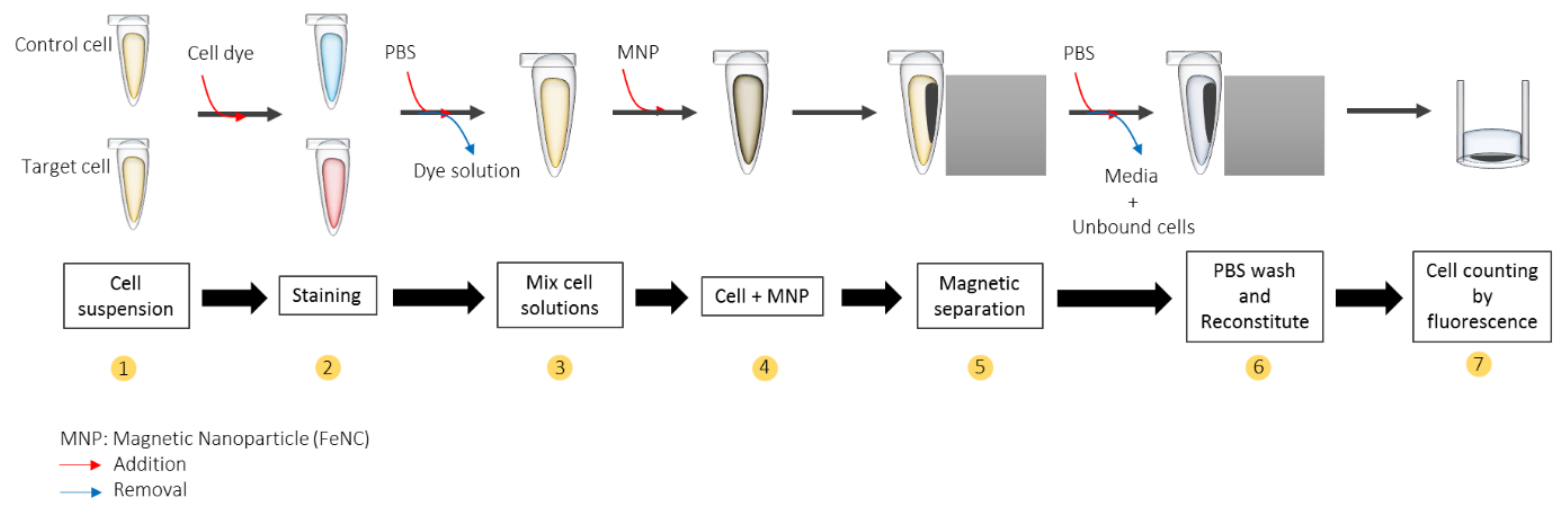

Scheme 2. Isolation of live CTC's using FeNCs assisted by magnetic capture.

amounts/ml). 200ul of cell suspension (known amount) was mixed with 200ul of control cells (same amount), and placed in a tube. Equal amounts of each target and control cell were also placed in separate tubes as non-treated control. $200 \mu 1$ of FeNC-Ab construct was then added to the tube with cells and incubated at $37^{\circ} \mathrm{C}$ for 2 hours (gently shaken every $5 \mathrm{~min}$ ). Following incubation, the tubes were placed next to a neodymium block magnet $(0.1 \mathrm{~cm}$ spacing) for $30 \mathrm{~min}$. The supernatant was carefully removed and the captured pellet was washed twice with $500 \mu$ l of $1 \mathrm{X}$ PBS using magnetic separation. The FeNC-Ab-Cell solution was then resuspended in $200 \mu \mathrm{l}$ of $1 \mathrm{X}$ PBS and transferred to flat bottom 96 well plate. To prevent any agglomeration a hydrophobic low retention $200 \mu 1$ tip was used to quickly mix and transfer without immersing the tip fully in solution. Cell counting was performed using an automated fluorescence based algorithm using Cytation5 imaging plate reader. 


\subsubsection{EMT conversion}

To induce EMT artificially in cells, $60 \mathrm{ng}$ of EGF (10 ng/ml) and $120 \mathrm{ng}$ of TGF $\beta$ (20 $\mathrm{ng} / \mathrm{ml}$ ) protein was mixed in $6 \mathrm{ml}$ serum free media and treated with A549 cells. Treatment was performed at $60 \%$ confluence in a $\mathrm{T} 25$ flask for $72 \mathrm{~h}$ in a $\mathrm{CO}_{2}$ incubator. Flasks were imaged at every $24 \mathrm{~h}$ using a microscope and images were obtained. At the end of the conversion, the flask was washed once with fresh RPMI media containing $10 \%$ FBS and twice with cold 1X PBS. Cells were then dislodged and used for magnetic capture.

\subsubsection{Western blot}

$1 \times 10^{6}$ HCC 827 and A549 cells at 70\% confluence were seeded in 6-well plates. Cells were treated in serum free media for a period of $72 \mathrm{~h}$ followed by whole-cell lysate preparation using lysis buffer containing $1 \mathrm{X}$ phosphatase inhibitor cocktail. Proteins were separated by $4-15 \%$ TGX-PAGE and were transferred onto nitrocellulose membrane for protein blot analysis. Membranes were washed with 1X TBST, blocked with 5\% BSA blocking buffer containing $0.5 \%$ NGS for $2 \mathrm{~h}$ and incubated with primary antibody overnight on a shaker at $4{ }^{\circ} \mathrm{C}$. Blots were then washed before and after incubation with secondary antibody, and developed with chemiluminescence system.

\subsubsection{Detection of cytokeratin}

Cells were captured by FeNCs were stained and fixed by adding $500 \mu 1$ of $4 \%$ paraformaldehyde and incubating for another 10 minutes at $37{ }^{\circ} \mathrm{C}$. Next, $500 \mathrm{ul}$ of $0.1 \%$ Triton X-100 was incubated in the cell solution for 5 min after staining and fixing. Rest 
of the steps were the same for FeNC treatment. After FeNC-Ab-Cell capture and washing once with $1 \mathrm{X}$ PBS, $500 \mu \mathrm{l}$ of $5 \%$ FBS solution in $1 \mathrm{X}$ PBS was added as a blocking agent for $30 \mathrm{~min}$. After removal of blocking agent, $400 \mu \mathrm{l}$ of Alexa Fluor 647 anti-Cytokeratin antibody $(2 \mu \mathrm{g} / \mathrm{ml})$ solution diluted in $1 \mathrm{X}$ PBS cells was added to the tube and incubated for $2 \mathrm{~h}$ at room temperature. Cells were then washed twice with $1 \mathrm{X}$ PBS and then resuspended in $200 \mu \mathrm{l}$ of $1 \mathrm{X}$ PBS. This solution was then quickly transferred to a 96 well plate for imaging and fluorescence based counting.

\subsubsection{Statistics}

All statistics were performed using One-way Anova on Minitab. Average values from triplicates were calculated and used for statistical analysis.

\subsection{RESULTS AND DISCUSSIONS}

\subsubsection{Properties of FeNC constructs}

FeNCs were synthesized by a high temperature thermal decomposition of iron acetylacetonate complex. This reaction generates $\mathrm{Fe}^{3+}$ ions that form iron crystal seeds at $295{ }^{\circ} \mathrm{C}$. By maintaining growth conditions, the seeds were transformed to iron nanocubes. Typically iron particles formed exist as a ferromagnetic state, however at around $20 \mathrm{~nm}$ particles undergo a transition from ferromagnetic to superparamagnetic. This transition allows the particles to be non-magnetized in an absence of an externally applied field. Results from SQUID analysis confirmed that the $20 \mathrm{~nm}$ nanocubes synthesized were indeed paramagnetic with a magnetic moment of $60 \mathrm{emu} / \mathrm{g}$ (Figure 44). The cubic shape imparted higher magnetic moments as compared to spherical 
counterparts. The particles were coated with a base polymer that had $-\mathrm{COOH}$ activations groups required for biomolecule functionalization on the surface. These particles were then stabilized using pegylation. This process makes them hydrophilic and extremely stable in different kinds of solvents. The overall coating thickness of these hydrophilic particles as shown in TEM images were up to $\sim 2 \mathrm{~nm}$ (Figure 41).

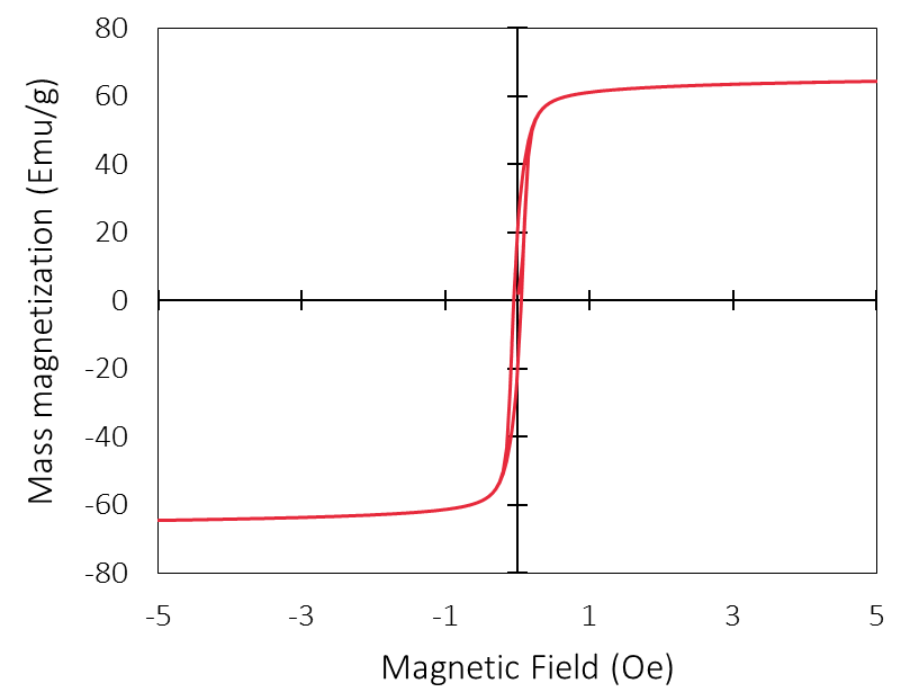

Figure 44. Magnetic hysteresis curve of FeNC obtained from SQUID measurements made at $2 \mathrm{~K}$.

Additionally, the hydrodynamic size increased confirming the conjugation to antibody on the surface. ELISA also confirmed the specific affinity of FeNC-Globin Ab to globin antigen (Figure 45). The specificity was linear with controls not being detected even at the highest concentrations. 


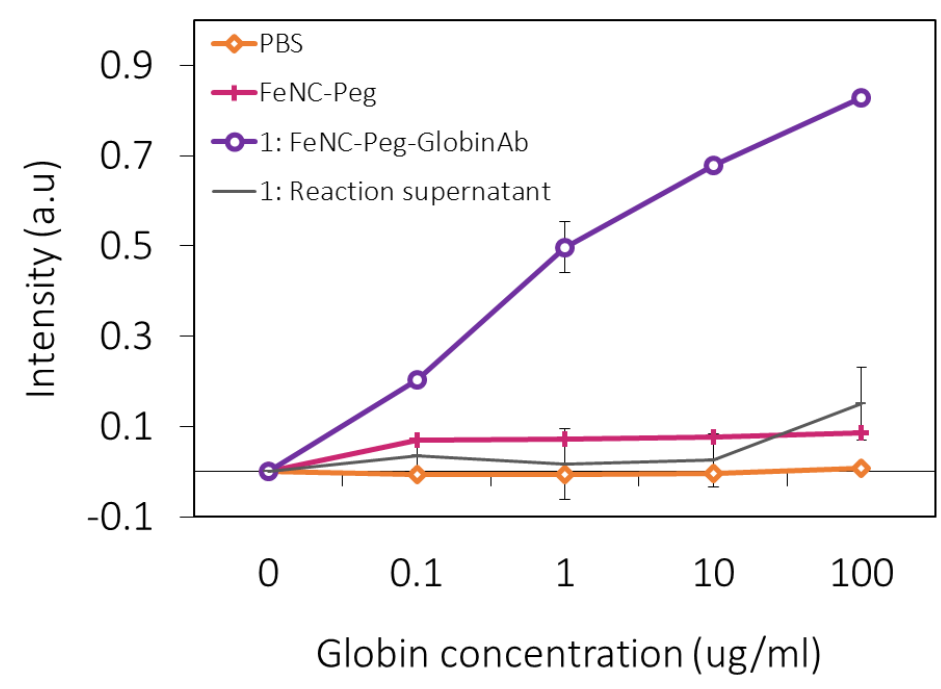

Figure 45. FeNC-Globin capture using a competitive binding assay.

After confirming the conjugation, binding affinity using globin, we conjugated EGFR and HER-2 targeting antibodies to the surface of the pegylated nanocubes to obtain highly uniform FeNC-HER/EGFR conjugates stable in aqueous solvent or media (Figure 46).

a

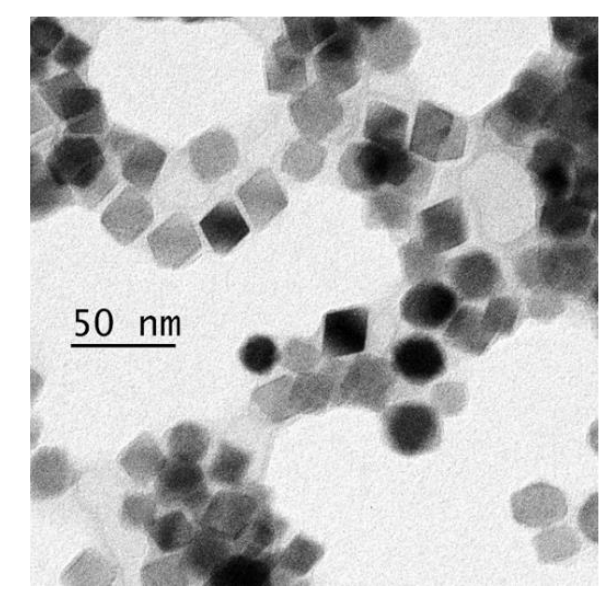

b

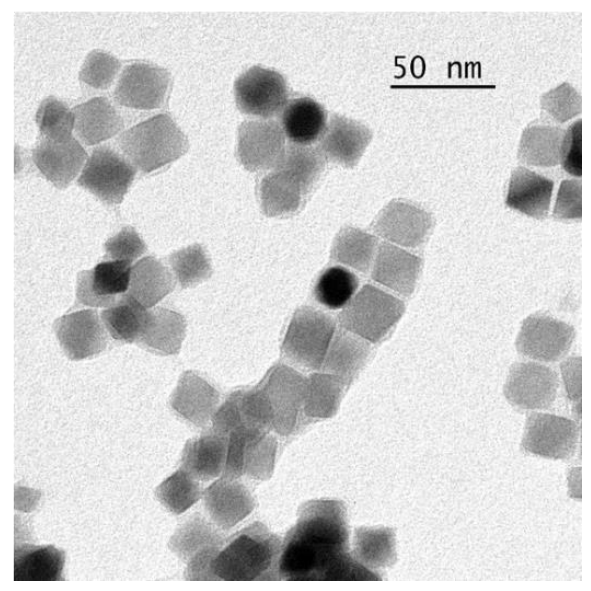

Figure 46. TEM images for (a) FeNC-HER and (b) FeNC-EGF. 
As a next step these FeNC-Ab particles (FeNC-EGF and FeNC-HER) used for targeting non-EMT NSCLCs. For the isolation of cells using FeNC-Ab, a robust magnetic capturing process was developed (Scheme 2). For this process a $250 \mathrm{lb}$ force magnet (Figure 47) was used to capture the cells tagged with FeNCs. This process had very high reproducibility to capture and characterize CTCs. In this method known amounts of target cells (HER2/EGFR marker positive) and control cells (negative) were mixed and the efficiency of the FeNC-Ab to isolate target cells was analyzed. The target cells were stained red while the control cells were stained with a blue dye for identification purposes (Figure 48). The amount of isolated cells in a given sample was detected using fluorescence that increases the detection sensitivity.

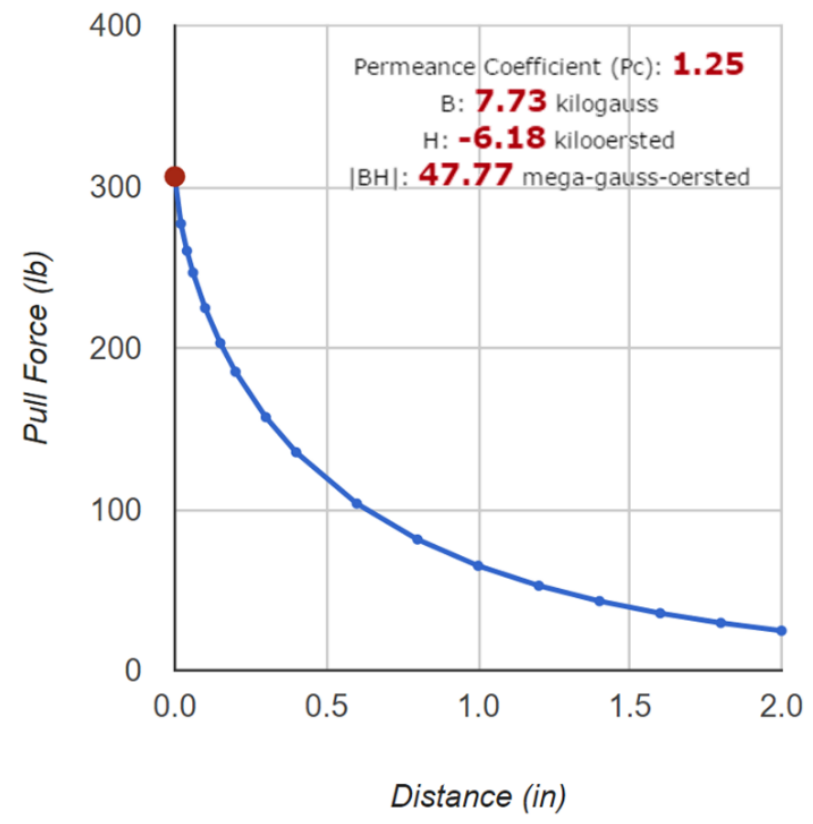

Figure 47. Properties of the external field applied for FeNC-CTC capture process. 


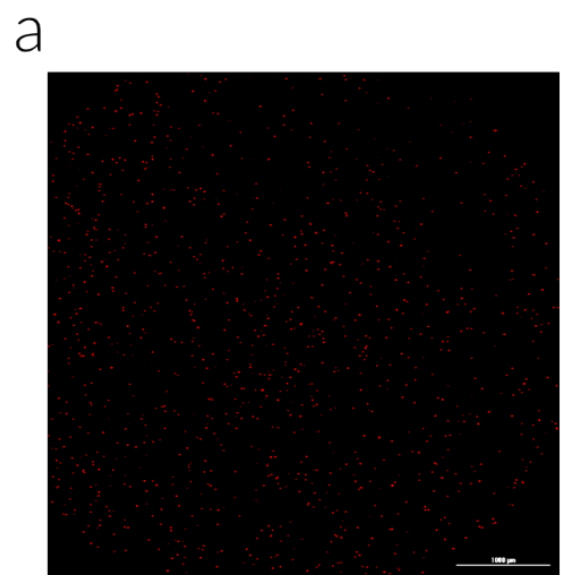

b

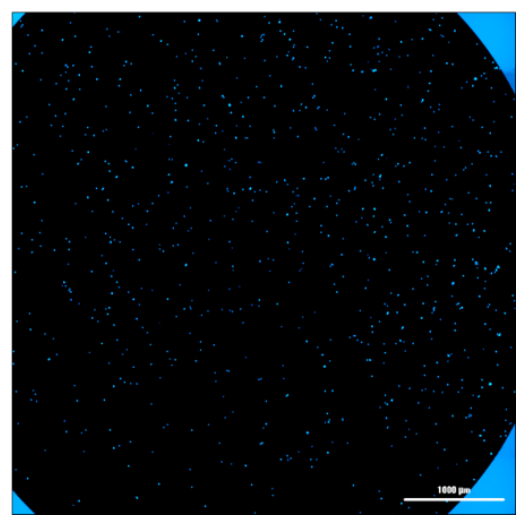

Figure 48. Cells were stained with (a) red dye using CellMask and (b) blue dye using Hoechst.

\subsubsection{Specificity and Sensitivity Tests}

To study ability of FeNC to capture NSCLC cells, we used HCC827 and A549 cells. Previous studies have shown HER-2 levels are upregulated in A549 KRAS mutant cells, while EGFR were shown to have very low expression ${ }^{93}$. Our western blot results confirm the same (Figure 49).

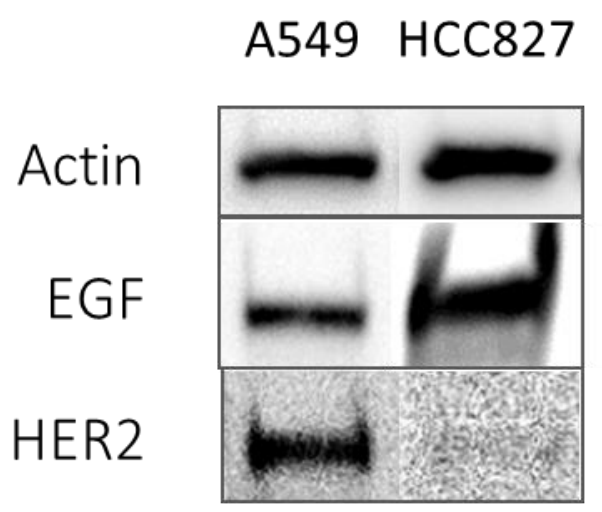

Figure 49. Western blot imaging shows that A549 cells were positive for HER-2, while HCC827 were not. Further the blot show that both cells were positive to EGFR, with HCC827 being more than A549. 
Therefore, we hypothesize that FeNC-HER will have the ability to capture more A549 cells as compared to HCC827 cells. In order to study the capture specificity of FeNCHER, we increased antibody loading on nanoparticle surface and captured target cells. Antibody amounts were double and tripled and they were compared against controls. Results show a linear increase in A549 cell capture as compared to HCC827 cells (Figure 50).
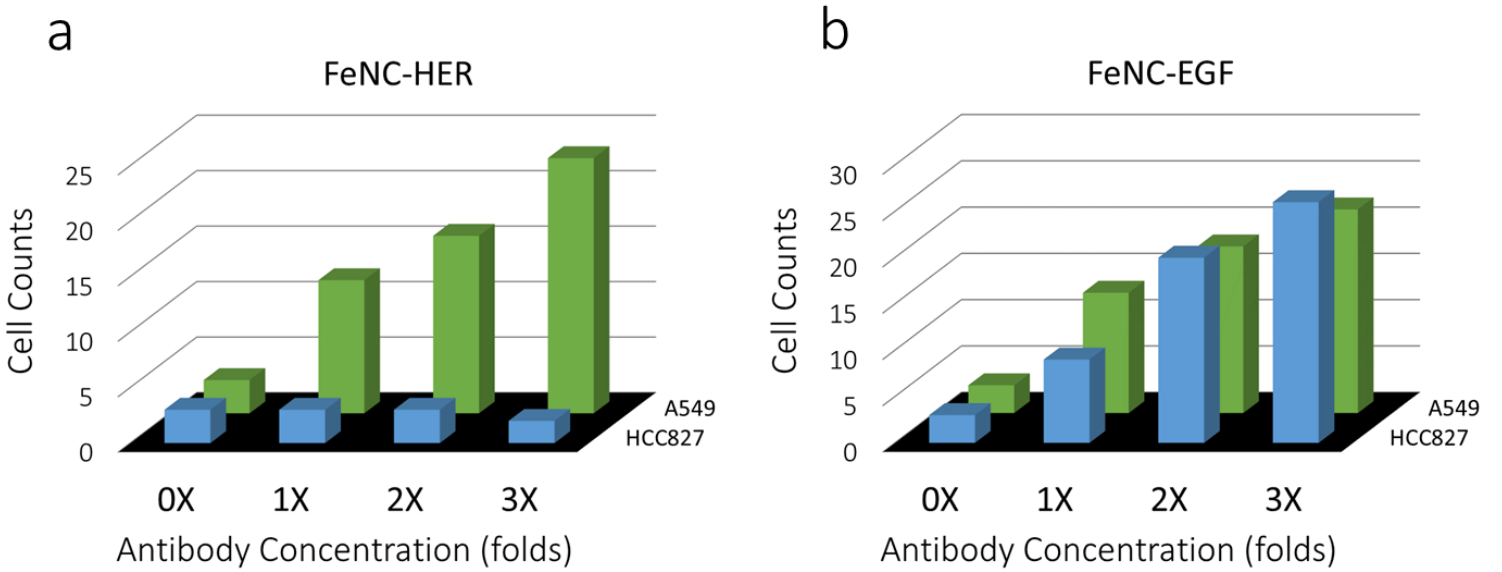

Figure 50. Specificity of (a) FeNC-HER and (b) FeNC-EGF towards HER-2 (A549) and EGFR (Hcc827/A549) expressing cells as function of surface antibody loading.

This data suggests that FeNC-HER was highly specific to HER-2 receptors. Separately we also studied the protein levels of EGFR in these cells. Previous studies have shown both cells express EGFR ${ }^{93,94}$. Therefore, FeNC-EGF should be able to capture both cells. However, our western blot analysis showed a relative decrease in EGFR expression in A549 cells as compared to HCC827. Our cell capture studies correlated well with EGFR expression; the amount of A-549 cells captured was relatively lower compared to HCC827 (Figure 50). The results obtained show the high specificity and sensitivity achieved in detecting EGFR specific cells. 
Detailed studies were performed to evaluate the sensitivity of the conjugates. We varied the concentration of nanoparticles used to capture cells. By doubling the amounts, we found that the number of cells captured were significant as compared to cells captured by non-specific binding to FeNCs without antibody (Figure 51). Overall, the analysis suggests a minimum concentration of $2 \times 10^{6}$ particles $/ \mathrm{ml}$ is required to achieve a high sensitivity in capturing cells without increasing non-specific binding.
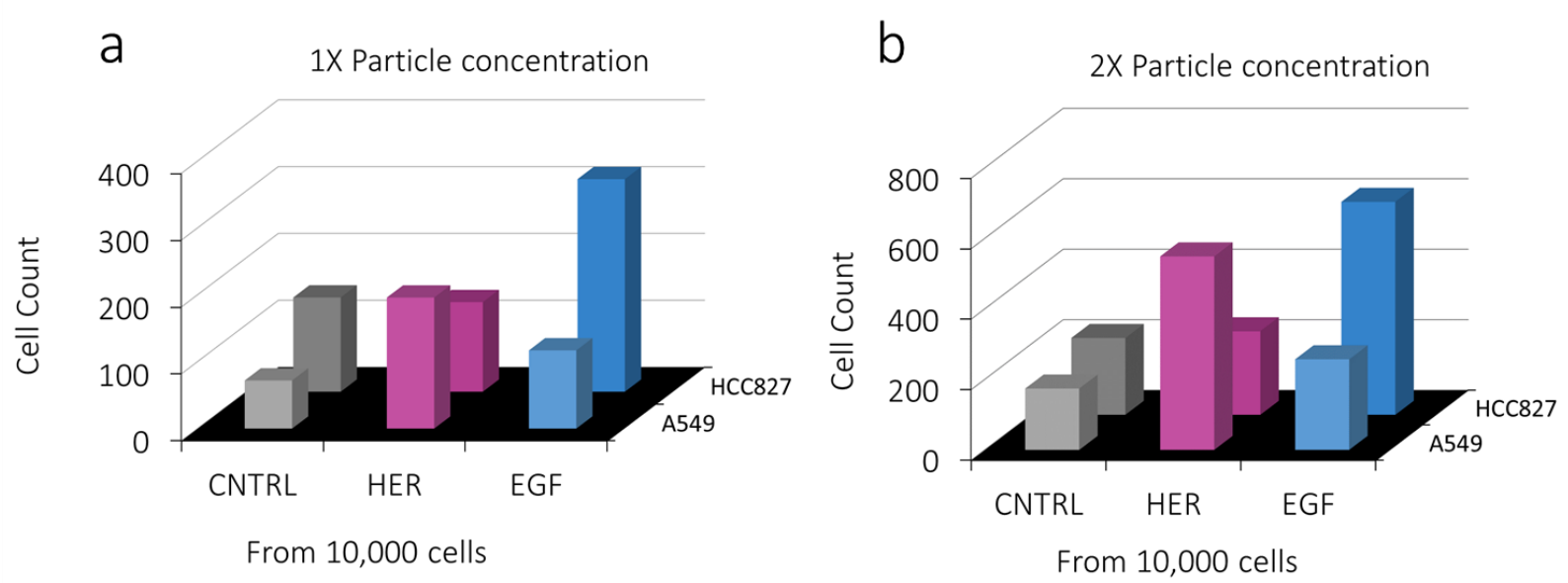

Figure 51. Sensitivity of FeNC-HER and FeNC-EGF towards HER-2 (A549) and EGFR (Hcc827/A549) expressing cells at different particle concentrations (a) $1 \times 10^{6}$ and (b) $2 \times 10^{6}$.

\subsubsection{Quantification in blood serum}

We conducted spiking experiments to evaluate the capturing ability of FeNC in serum samples. For this purpose, we spiked a mixture of A549 and HCC827 in pig serum, instead of $1 \mathrm{X}$ PBS. In this experiment the amount of cells captured from a known mixture of A549 and HCC827 cells using FeNC-EGF, FeNC-HER and FeNC control was compared. Based on our specificity and sensitivity tests we hypothesized that FeNC-HER would show considerable difference in capturing A549, while FeNC-EGF should capture both. Therefore, the capture efficiency from 10000, 1000 and 100 spiked 
cells were tested for each construct. Results showed that FeNC-HER and FeNC-EGF maintained their specificity towards HER-2 and EGFR receptors in serum conditions (Figure 52). In fact, FeNC-HER was able to capture more A549 cells in all three tests (Figure 52). Data suggests the specificity is more apparent when the amount of cells are decreased. On the other hand, FeNC-EGF was able to capture both cells, HCC827 cell capture numbers were relatively higher. The results demonstrate the capability of magnetic nanocubes to detect spiked CTCs in physiological media. As a next step, we further tested ability of FeNC-Ab in capturing EMT CTCs.

a

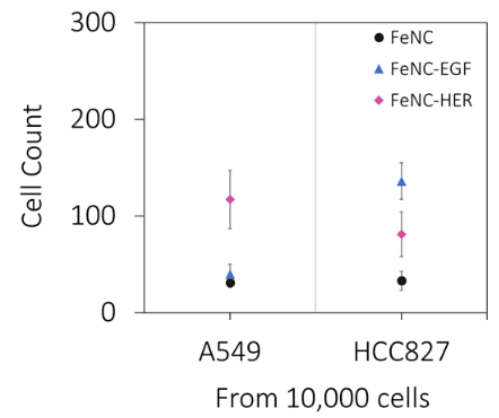

b

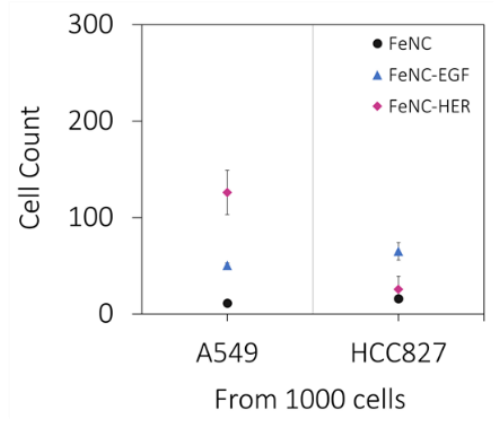

C

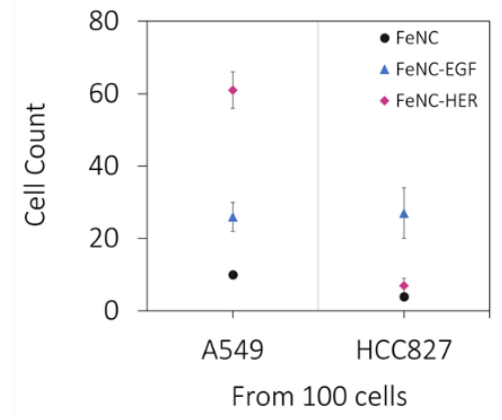

Figure 52. Cell capture from A549-HCC827 mixture spiked in serum using FeNC-HER and FeNC-EGF constructs. FeNC was used as a control to study non-specific binding. For this study (a) $10^{4}$, (b) $10^{3}$, (c) $10^{2}$ cells were spiked and captured using FeNC-ab constructs.

\subsubsection{Investigating effect of EMT pathway on epithelial markers}

Several clinical studies before and after drug treatment have shown that under EMT upregulation, cells lose their epithelial markers such as EpCAM or cytokeratin and gain 
mesenchymal invasive characteristics ${ }^{95}$. These characteristics favor proliferative and survival abilities in cancer cells. Therefore, EMT would in turn upregulate other known oncogenic markers such as EGFR and HER-2 in cell. Based on this information we hypothesized that cells will lose EpCAM and cytokeratin, but will not lose EGFR or HER-2 markers on the cell surface. To study this effect in vitro, we artificially induced EMT in A549 cells. As soon as we induced EMT, cell imaging confirmed the change in cellular morphology (Figure 53). Results show EMT induction allows cells to be more plastic and elongate in nature. These features are frequently seen in earlier studies and explain the cause for metastases ${ }^{80}$.
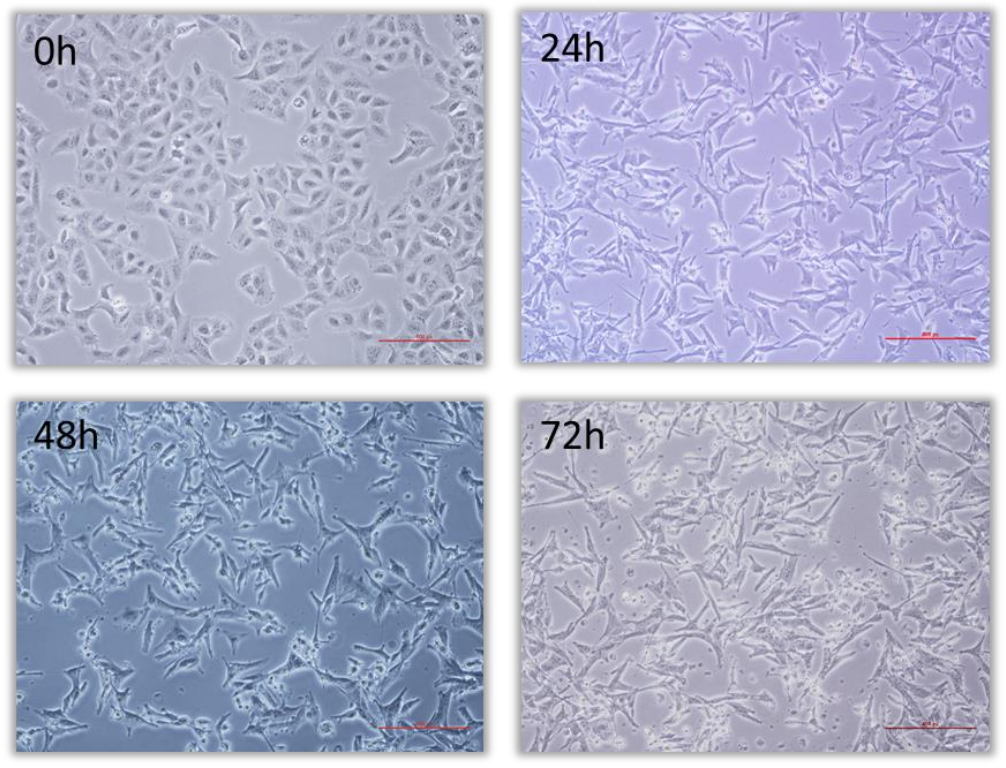

Figure 53. Microscopy for EMT activated cells indicate an elongated elastic morphology with reduced surface binding signifying loss of epithelial binding markers used for adhesion (40x; bright field) 

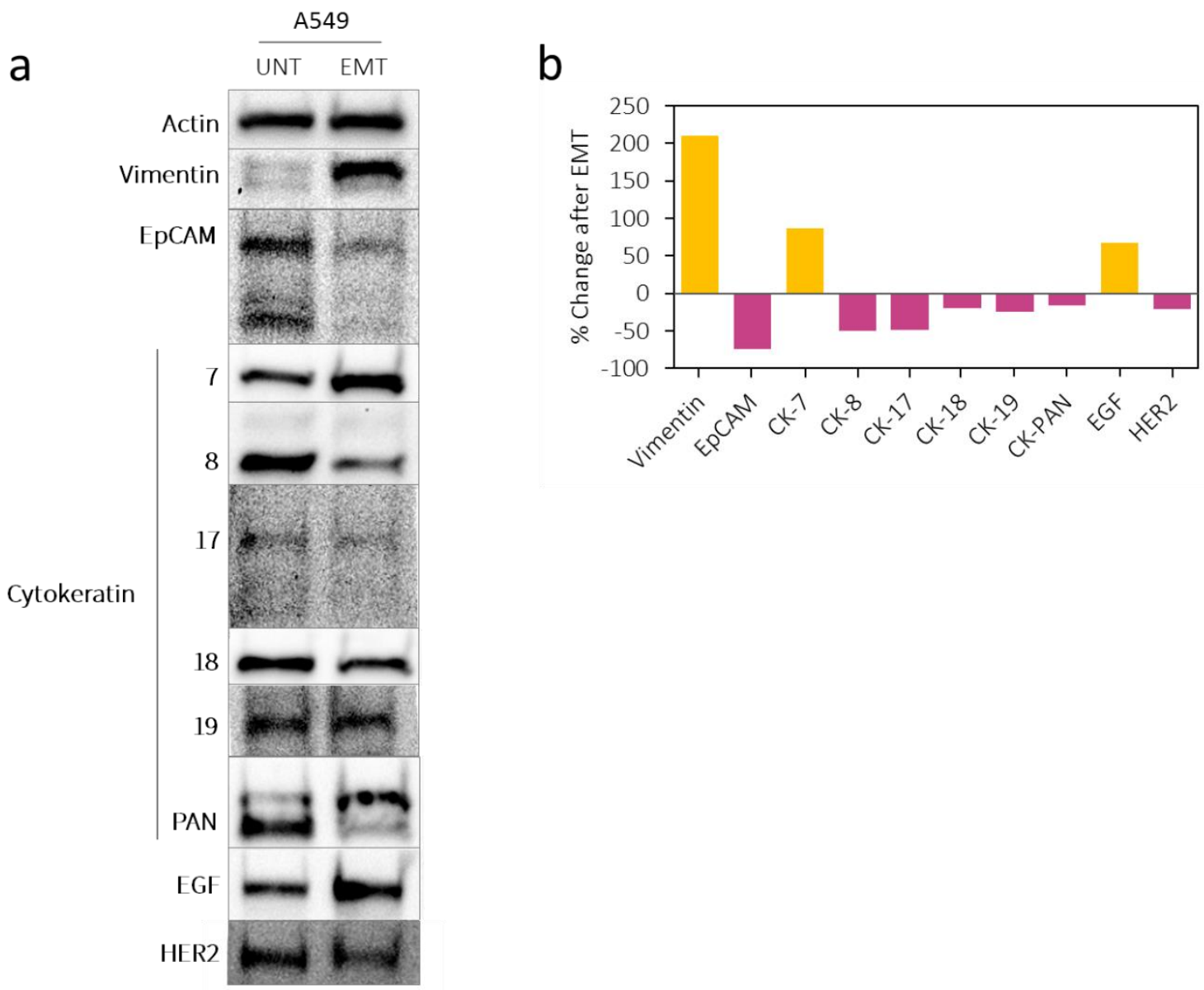

Figure 54. Western blotting for analysis of EMT marker Vimentin, epithelial markers EpCAM and cytokeratin, and oncogenic overexpressed markers EGF and HER-2 in normal A549 and EMT activated A549 lung cancer cells.

Furthermore, we isolated cell lysates and analyzed the total protein levels using western blotting. In the blot analysis, EMT-cells showed an upregulation in Vimentin that is associated with regulation of downstream mesenchymal proteins such as $\mathrm{N}$-cadherin and E-cadherin (Figure 54). The data indicates that EMT pathway has been activated by treatment of TGF $\beta$ and EGF growth factors. Remarkably, results show a drastic decrease in epithelial markers in EMT cells compared to non-EMT cells. Specifically, EpCAM was downregulated by more than 70\% in EMT-cells (Figure 54). Additionally, cytokeratin markers such as $8,17,18,19$, and PAN were all downregulated by $15-50 \%$ 
in EMT-cells confirming that cells undergoing EMT lose epithelial markers (Figure 54). Remarkably, we see 70\% upregulation in EGF protein in these cells (Figure 54). Though HER-2 decreased in EMT-cells the downregulation was not so significant. We indeed observe considerable levels of HER-2 protein after EMT conversion. Therefore, both EGFR and HER-2 could form effective targets for capturing CTCs. Based on these results, we anticipate that our FeNC-Ab constructs would be able to capture EMT-CTCs devoid of EpCAM expression.

\subsubsection{Detection of EMT status and EMT-CTCs}

After inducing EMT in drug resistant A549 human lung cancer cells, we investigated the possibility of detecting the loss of epithelial markers using our magnetic capture technique. We investigated the EMT status of both non-EMT and EMT-CTCs using FeNC-EGF and FeNC-HER constructs, by detecting the cytokeratin levels displayed in the captured and isolate cells. We compared the number of nuclei (stained with blue dye) to that of number of cells with cytokeratin (stained with red dye) to quantify the change in cytokeratin expressing cells. Results show that EMT-cells isolated using both the constructs had low expression of cytokeratin (Figure 55). The results corroborate the data from our protein analysis in A549 cells and proves the ability of FeNC-Ab to capture and isolate cells for further biomarker analysis and for DNA isolation from CTCs. 

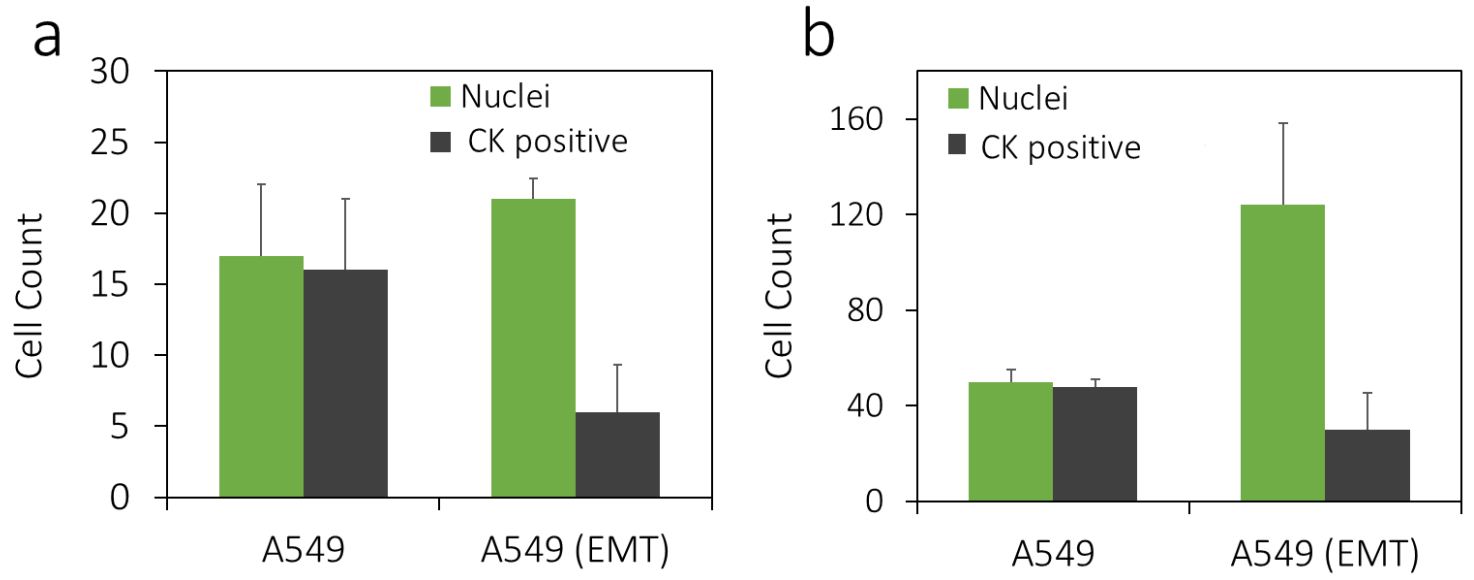

Figure 55. EMT-cells captured by (a) FeNC-HER and (b) FeNC-EGF show decreased epithelial cytokeratin expression.

A sharp increase in cell capture counts in EMT-cells was observed. To further investigate this increase, we spiked 100, 50 and 10 cells in serum and quantified the amount of cells captured by FeNC constructs. Results show that FeNC-EGF was able to capture both non-EMT and EMT cells (Figure 56).

a

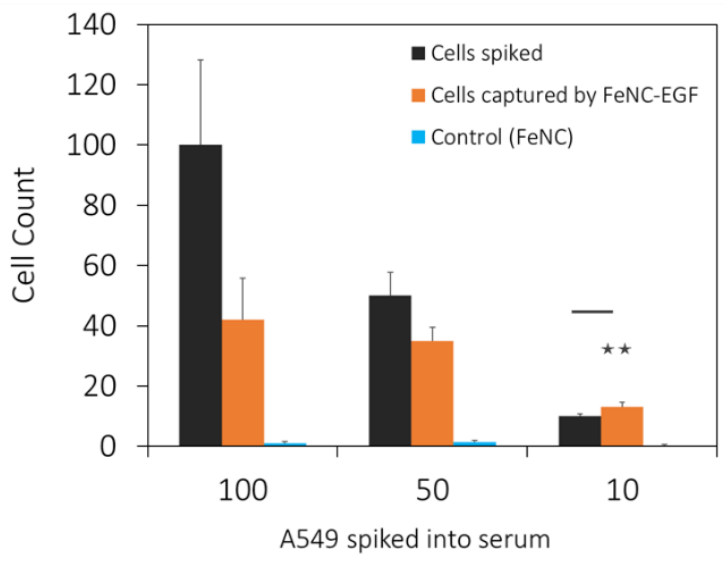

b

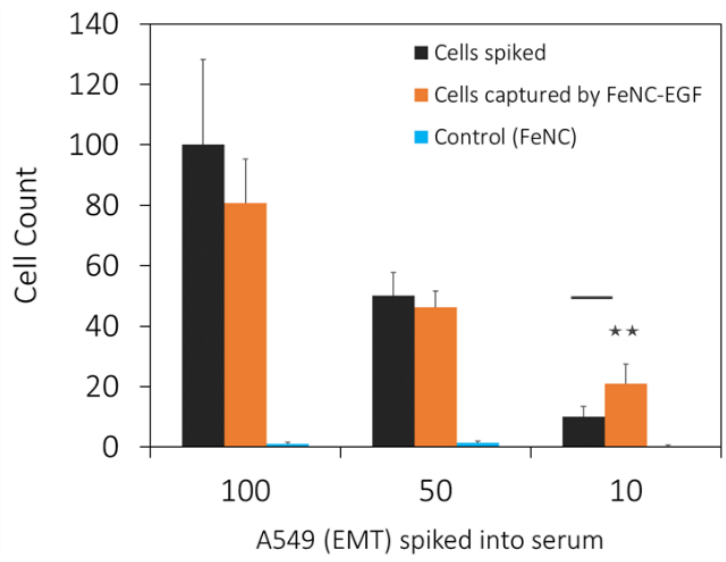

Figure 56. Amount of cells captured by FeNC-EGF (a) before and (b) after EMT. Results indiciate FeNC-EGF can capture as fewer as 10 cells without any loss in sensitivity after EMT. ${ }^{\star \star} \mathrm{p} \leq 0.01$, and the values were analyzed using one-way anova. 
Interestingly the capture efficiency was 2 fold in EMT-cells. This increase can be attributed to EGF protein upregulation. Similarly, we also observed the ability of FeNCHER to capture cells before and after EMT conversion (Figure 57). Additionally, we again noticed an increase in capture of EMT cells. These results suggest that, by targeting EGFR and HER-2 markers, we can capture CTCs efficiently irrespective of cells losing their epithelial markers and can serve as a robust approach for detecting CTC prior and during drug treatment. Furthermore, the results indicate, FeNC-Ab conjugates can capture as fewer as 10 cells. These conjugates provide an unique opportunity to be used in clinical setting, where capturing very few cells is key component in NSCLC diagnosis and treatment.

a

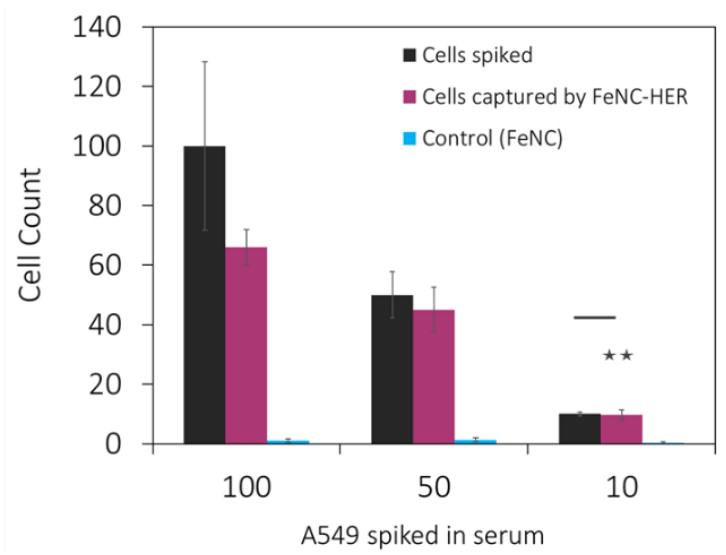

b

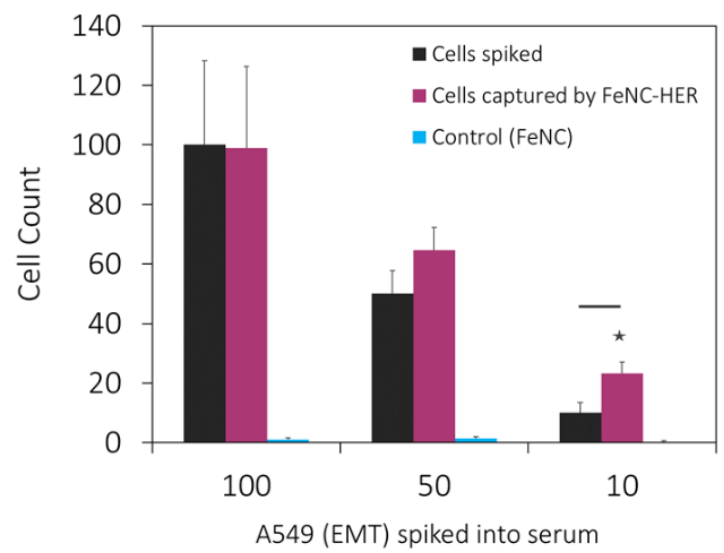

Figure 57. Amount of cells captured by FeNC-HER (a) before and (b) after EMT. Results indiciate FeNC-HER can capture as fewer as 10 cells without any loss in sensitivity after EMT. ${ }^{\star} \mathrm{p} \leq 0.05$, and the values were analyzed using one-way anova. 


\subsection{CONCLUSION}

In this study we synthesized antibody conjugated $20 \mathrm{~nm}$ paramagnetic iron nanocubes. We characterized the constructs and tested their ability in capturing spiked NSCLC CTCs. We found that the conjugates are extremely efficient in capturing these cells based on EGFR and HER-2 targets. Furthermore, we confirmed the loss of epithelial markers such as EpCAM and cytokeratin in cells undergoing EMT. The constructs showed very high EMT-cell capture efficiency. Additionally, the data suggests the high specificity of these conjugates to isolate and detect cells undergoing EMT. This provides an excellent example of the use of paramagnetic particles as a versatile tool for CTC capture and isolation based on receptor targeting. The methods established in this study allows for design and development of nanoparticle based non-invasive diagnostic kits. 


\title{
How to structurally collapse nanoparticles for molecular release using external stimuli?
}

\author{
$>$ Designing nanostructures to atomically rearrange in presence of femtosecond \\ pulsed laser
}

\begin{abstract}
Presently, nanomedicine strategies are slowly transitioning from a sustained drug delivery models to a controlled delivery model which can be far more effective and precise. Controlled delivery often involve molecular release from nanoparticles via external stimuli such as photonic or ultrasound energy. Therefore, it is extremely important to design functional nanostructures which can respond to a laser source with high sensitivity and low energy threshold. Current standard involves a microsecond or a nanosecond laser source which can lead to unwanted photothermal effects due to particle melting. An answer to this issue requires use of ultrashort femtosecond photonic source which can affect only electrons of a nanocrystal while leaving the lattice cold. This means particles can absorb and transfer energy before entering a melting phase. However sub-picosecond photonic interaction with nanoparticles remains a grey area and needs to be experimentally proven in agreement with theory. Also the mechanisms involved in energy absorption and thresholds are not fully understood. As a solution, functional nanocubic structures were developed, and cubic shape was examined for efficient energy confinement. Surface plasmon resonant (SPR) modes of the cubic nanoparticle were tailored to resonate and couple with pulsed laser source making the process extremely wavelength selective and requiring very less energy to cause a non-thermal particle modification. This modification allowed controlled release of biomolecules.
\end{abstract}




\section{METHODS}

Cubic nanocubes, nanoboxes and nanocages with specific structures were synthesized to study near field focusing of light energy using a femtosecond ultrashort pulse laser. Particle shape and its SPR modes were investigated by finite domain time domain (FDTD) simulation and key structural loci were selected for examination. Energy absorption was recorded as a function of SPR transformation monitored via transmittance and absorption optical spectroscopy. Particle modifications were visually recorded using transmission electron microscopy (TEM). Subsequent elemental analysis and crystallography was performed using Energy dispersive spectroscopy (EDS) and high-angle annular dark-field imaging (HAADF). Activation thresholds were correlated with shape modification via size distribution and single nanoparticle hyperspectral Rayleigh scattering spectroscopy. Atomic rearrangement was examined via high resolution TEM and non-thermal effects were correlated with energy absorption. Inertness of laser source and particle modifications were studied in cancer cells by MTT cell viability assays. Finally, molecular release of surface conjugated peptide was monitored using transmittance optical spectroscopy of surface peptide coating.

\section{RESULTS}

Highly controlled particle deformation was achieved under ambient environments by shape enhanced plasmonic hot-spots capable of confining incident optical fields through near field energy focusing. In this study, dynamic control over structural transformation of precisely modified cubic nanostructures; namely cubes, boxes and cages were achieved with a sub $50 \mu \mathrm{j}$ incident energy input. We detected three types of ultrafast light-matter interactions each characterized by geometry specific activation thresholds, and our observation suggests direct 
causation between a localized plasmon field and excitation energy required, leading to ultrafast sub-10 nm maskless photolithography by a non-thermal mechanism. Furthermore, these thresholds can be used for molecular release without causing off-side photothermal effects within cells. 


\section{CHAPTER 3}

\subsection{INTRODUCTION}

Current drug delivery systems largely rely on sustained release of drugs. For this purpose, nanoparticles are designed to allow drugs to release with time ${ }^{7,96}$. The sustained delivery is possible through the use of porous or biodegradable particles ${ }^{97,}{ }^{98}$. The drug concentration in circulation is thereby increased during the leaching process but is nonselective. Due to the non-selective nature, the drug often interacts with other organs and healthy tissues, leading to toxic side effects. A solution for this issue would require targeted therapy using an active delivery technique. Using this approach, cancer cells, tissues or tumors within the body can be targeted by nanoparticles. The nanoparticles can then be used to release drug only at the cancerous site in order to reduce side effects. In order to achieve this, the nanoparticle itself or its surface has to be functionalized with a responsive polymer. For example, several studies have demonstrated the use of smart polymers like hydrogels, pNIPAM, PMMA or dendrimer based structures over nanoparticles for controlled delivery ${ }^{99-102}$. These polymers such as pNIPAM (poly(N-isopropylacrylamide)) were used to control gel-to-sol transition state of the polymer on the surface. Such polymers can swell and increase its porosity when exposed to altered cellular $\mathrm{pH}$ or body temperature to allow drug release from within the nanoparticle ${ }^{103}$. However, use of polymers increase the hydrodynamic size of nanoparticle as well as impart toxicity ${ }^{104}$. Additionally, these polymers can increase steric hindrance to surface markers that target cancer cells. Instead, we can limit these deficiencies by using external agents to cause changes to the nanoparticle's physical state. As a result, using an external source to excite nanoparticles is viewed to be a more selective and sensitive approach for a targeted controlled release of 
$\operatorname{drug}^{105-107}$. Using this approach, energy from different sources of radiation can be used to modulate particle behavior. However, development of such technologies requires a functional nanoparticle that can respond to external radiation. Furthermore, the mechanisms of how nanoparticles respond and behave with external radiation is not fully understood. Therefore, finding a mechanism of release using external energy sources can play an important role in the development of a non-toxic controlled drug delivery technique.

\subsubsection{Smart nanomaterials for controlled drug delivery}

Unlike bulk metallic materials, nanoparticles have unique properties due to their size and shape ${ }^{108-110}$. Nanoparticles have been shown to exhibit highly specific light absorption, fluorescence as well as energy storage properties ${ }^{111-113}$. Several studies have experimentally shown that nanoparticles can absorb different kinds of radiation such as laser or ultrasound ${ }^{105}$. By absorbing radiation, the nanoparticle surface or structure could be modified, and therefore allow drug release. For example, core-shell nanoparticles and thermoresponsive liposomes were shown to respond to ultrasound energy $^{114,115}$. In that study, ultrasound energy was converted to chemical or heat energy to cause an activation. However, the merits of using lasers include highly localized treatment, uni-directional energy focusing and highly specific wavelength of operation. For example, pNIPAM coated gold nanocages were activated using NIR laser with a specific wavelength to heat and cause polymeric swelling ${ }^{116}$. Although these studies used external energies for a more directed controlled release, the release was still dependent on polymeric surfaces or heat energy that can affect nearby tissues. 
Moreover, the results from laser based studies show presence of nanoparticle heating that can cause thermal damage to tissues ${ }^{117-119}$. Therefore, for a non-photothermal approach it is imperative to utilize laser wavelengths that are not only transparent to tissues (near infrared (NIR) region), but do not allow intense nanoparticle heating. Moreover, to reduce these side effects, it is important for radiation to be absorbed at very low energies and cause change in nanoparticle behavior without the use of polymers. This technique would require functional nanoarchitecture to further increase light absorption characteristics.

Recent studies have shown that nanoparticles with high aspect ratios such as cubes or rods are excellent light absorbers and reflectors ${ }^{120-124}$. These qualities suggest that light interactions with anisotropic structures are highly specific. High aspect ratios allow very sharp features at the nanoscale. Similar to bulk physics, sharp features can enhance electromagnetic fields (EMF) at the nanoscale ${ }^{125,126}$. The resulting EMF at the surface of these nanoparticles have a characteristic energy wavelength. Because, light itself is an electromagnetic field it can interact with these features at the nanoscale. By modulating the wavelength of incident light the interactions may be more pronounced, and lead to high absorption of light. This increase in efficiency can be described by the Purcell factor, $\eta_{P}$ (Equation 1) ${ }^{127}$.

$$
\eta_{\mathrm{P}}=\frac{\gamma_{s p}}{\gamma_{r a d}+\gamma_{e w}},
$$

Here, $\gamma_{s p}, \gamma_{r a d}, \gamma_{e w}$ are decay rates associated with surface plasmons, radiative energy and bound modes. Thus, efficiency is a function of surface plasmons that is a dependent on structural shape. Therefore, using cubic or rod shaped particles we can achieve better energy absorption characteristics. However, finding the ideal shape requires an 
understanding of how these electromagnetic fields are generated on these particles and how they interact in different shapes.

\subsubsection{Localized surface plasmon resonance}

Particles at the nanoscale have very high surface to volume ratio thereby increasing relative atomic concentrations at the surface than the core ${ }^{128}$. Because of this property, nanoparticle characteristics is dominated by surface properties. The size decrease also leads to increase in energy band gap properties relative to bulk metals ${ }^{129}$. This increase in band gap signifies an increased incident energy required to excite electrons from the valence band to conduction band. For example, as bulk gold metal is reduced to a nanoparticle, a distinct blue shift is observed in color as higher energy light is required for electron absorption ${ }^{130}$. Further, in nanocrystalline materials, the electronic states are quantized (discrete energy levels) due to this change in band gap properties. However, nanoparticles made of noble metals such as gold and silver have free d-orbital electrons that are spatially confined ${ }^{131}$. As such, once particle size decreases below $50 \mathrm{~nm}$ (mean free path of an electron), the absorption properties increase while scattering power decreases ${ }^{132,133}$. This effect is explained by Mie scattering and is caused by the spatial confinement of free electrons on the surface of the nanoparticle to form an electron cloud $^{134}$. This electron cloud can have a specific wavelength depending on the size and shape of the nanoparticle. More importantly, the electron cloud can interact with incident light and absorb energy. For instance, if the wavelength of incident light matches with that of the electron cloud it would lead to absorption causing an oscillation of the electron cloud. This oscillation is referred to localized surface 
plasmon resonance (LSPR) relative to the positive atomic core ${ }^{130}$. The coherent oscillations are frequently associated with the Drude model to understand the linewidth of the plasmon resonance. For gold and silver, it is given by (Equation 2) ${ }^{135}$ :

$$
\Gamma=\gamma_{b}+\frac{\mathrm{A}_{v_{F}}}{L_{e f f}}
$$

Where $\gamma_{b}$ is the bulk damping, $\mathrm{A}$ is a constant, $v_{F}$ is the Fermi velocity and $L_{\text {eff }}$ is the effective electron path length. Subsequently, LSPR is responsible for electromagnetic field enhancement around the nanoparticle surface. When nanoparticles have high aspect ratios, it can contribute to directional LSPR. For example, gold nanorods have a longitudinal and a transverse LSPR mode. The LSPR modes in these asymmetric nanorods are considered decoupled due to the energy gap ${ }^{136}$. Therefore, the asymmetric shape gives rise to a polarization dependent excitation property ${ }^{137}$. However, special anisotropic structures such as cubic nanoparticles have a symmetric shape that can lead to coupled LSPR modes. Unlike rods, this hybridization leads a broad absorbance peak in the UV region and gives rise a polarization independent excitation ability ${ }^{138}$. Furthermore, hollow cubic structures can have enhanced surface fields due to coupling between its interior and exterior LSPR modes ${ }^{139,}$ 140. Such unique characteristics can essentially enhance inter-mode energy exchange ${ }^{141}$. Interestingly, cubic particles can absorb energy specifically in the NIR region ${ }^{39,142}$. Thus these particles not only are efficient in absorbing light but specifically absorb at wavelength s considered safe for tissues $^{143}$. Therefore, based on these data, a cubic nanoparticle less than $50 \mathrm{~nm}$ should be designed for the use of controlled activation by photonic radiation. 


\subsubsection{Photon-electron-phonon (light-matter) interaction}

In a previous study, cubic nanoparticles were revealed to be an excellent NIR energy absorber ${ }^{144}$. This study utilized a nanosecond laser tuned to the absorption wavelength of the particle. However, such studies also showed that, the laser energy was converted to heat. The high heat generation led to photothermal killing of cells ${ }^{145}$. As mentioned before, this is not part of the design criteria. In order to design nanoparticles that absorb energy but do not result in high photothermal conversion we require an understanding of how light energy is absorbed and converted to heat in nanoparticles. It is postulated that photons are directly captured by electrons to cause excitation, and subsequently increase the average electron temperature within the nanocrystal ${ }^{146}$. The electron excitations has been postulated to be associated with ultrashort electron oscillations and the ensuing ionic collisions explained by the Brunel effect (Equation 3) ${ }^{147}$ :

$$
V_{o s c}=\frac{e E}{m \omega},
$$

where $V_{\text {osc }}$ represents vacuum oscillation, e and $\mathrm{m}$ are the charge and mass of the electron, and $\mathrm{E}$ and $\omega$ are the electric field and frequency of the incoming electromagnetic radiation. In these crystals, the absorbed energy is converted to kinetic energy (electron heating) due to inverse Bremsstrahlung, leading to a fermi distribution of electrons that gradually changes to a maxwell distribution ${ }^{148}$. This effect eventually forms an electron plasma on the surface of the nanoparticle ${ }^{149}$. The heat capacity of this degenerated gas is given by (Equation 4$)^{147}$ :

$$
C_{e} \approx \frac{\pi^{2} E_{b}}{6 E_{f}} \kappa_{\mathrm{B}},
$$


where $E_{b}$ and $E_{f}$ are the binding and fermi energy and $\kappa_{\mathrm{B}}$ is the Boltzmann constant. Using equation 4 , the changes have been predicted to occur in the range of 1-20 $\mathrm{fs}^{16}$. Eventually, the energy is transferred to the lattice, known as electron-phonon relaxation that occurs within the range of several picoseconds ${ }^{147,150}$. This relaxation is influenced by the electron emission process described by the modified Dushman equation (Equation 5) ${ }^{151}$,

$$
Q_{R D}=\left(\frac{\pi D^{2} \phi}{e}\right) J_{\mathrm{RD}}, \quad \text { (Equation 5) }
$$

Where $Q_{R D}$ is the cooling rate due to thermionic emission, D reflects particle diameter, $\phi$ is the cathode work function, $e$ is the electron charge and $J_{\mathrm{RD}}$ is the emmited current density. The lattice energy is finally absorbed by surrounding medium in the range on nanoseconds ${ }^{147}$. Therefore, if the duration of relaxation is larger than the duration of laser pulse, it would lead to specific heating of electrons in the crystal lattice without heating up the lattice itself. Conversely, when nanoparticles were exposed to a nanosecond pulse, particle lattice would experience high heat conversion during the duration of laser pulse. Several studies in rods and spheres have shown this leads to particle melting due to high crystal temperatures ${ }^{152,153}$. In other words, if the pulse duration was in the range of femtoseconds (ultrafast light pulses), the average temperature of the lattice through the duration of pulse will be unchanged, that is referred as "cold lattice". The atomic displacements in this cold lattice can be expressed by change in atomic vibrational amplitude $\Delta \mathrm{q}_{\mathrm{k}}(\text { Equation } 6)^{147}$ :

$$
\Delta q_{k}=\frac{F_{t h, k}}{2 \omega_{0} \gamma M} \exp \left\{\iota\left(\omega_{0}^{2}-\gamma^{2}\right)^{1 / 2 t}-\varphi\right\}
$$

where $\gamma$ the damping factor, $\omega_{0}$ is the phonon frequency, $F_{t h, k}$ is the atomic force experienced and $\varphi$ accounts for the change in amplitude. Any changes occurring at this 
stage would theoretically be athermal. As the electrons are exited during the duration of the pulse alone, it would lead to very high absorption efficiency. At this stage, electrons will attain maximum energy reaching a temperature $T_{e^{-}}^{\max }$ given by (Equation 7) ${ }^{147}$ :

$$
\kappa_{\mathrm{B}} T_{e^{-}}^{\max }=\left[\frac{4 \epsilon_{f} A F\left(t_{p}\right)}{\pi^{2} n_{e} l_{s}}\right]^{1 / 2},
$$

where $A F$ the is the absorbed fluence, is $n_{e}$ the electron number density and $l_{s}$ is the skin layer of the material interface. Resulting heat that is inversely proportional to the skin layer will induce localized multiphoton and avalanche ionization. The electrons eventually will undergo electron-phonon relaxation succeeded by phonon-phonon relaxation that will increase the average lattice temperature. But the particles undergoing relaxation will not be exposed to any additional radiation if the pulse duration is shorter than the relaxation time. Thus, during this stage the particle will not attain a melting stage immediately. That is, electrons will establish a Fermi-Dirac distribution and the phonon state will not reach the high $t_{\text {tail }}$ of the maxwell distribution $(\text { Equation 8) })^{154,155}$.

$$
f(\varepsilon)=\frac{2 m}{(2 \pi \hbar)^{3}}\left(1+\mathrm{e}^{(\varepsilon-F) / \kappa_{B} T_{e}}\right)^{-1}
$$

where $\varepsilon$ is electron energy; $F$ is Fermi energy that is influenced by material, geometric shape, and size of nanoparticle; $T_{e}$ is electron temperature. This relaxed state with high electron temperatures, will lead to non-linear effects. Therefore, by using a laser pulse shorter than picoseconds we can conceivably achieve a transient non-thermal particle alteration. However, this remains to be experimentally proven. Furthermore, femtosecond laser effects and mechanism in cubic particles remains unexplored. 


\subsubsection{Ultrafast shape assisted structural deformation}

By tuning the wavelength of femtosecond light pulses we can selectively excite nanoparticles without affecting the surrounding medium. Studies have experimentally predicted these effects to be largely non-thermal in nature ${ }^{156-158}$. A non-thermal mechanism can lower the fluence needed for particle excitation, thereby reducing amount of incident energy. In nanoparticles with high aspect ratios, for instance cubes, the EMF enhancements might allow further absorption of light, and a reduction in required fluence ${ }^{159}$. This means shape plays an important parameter in absorption of laser pulses. Therefore, shape of the nanoparticles that dictate LSPR modes can theoretically allow particle deformation at precise points on the nanoparticle without affecting the rest of the nanoparticle. Previous studies have shown this effect in nanoparticles with exposure to femtosecond light radiation ${ }^{159}$. These studies have observed fragmentation of nanoparticles that are postulated to follow a (1) coulomb or (2) electrostatic mechanism of crystal deformation ${ }^{147,}{ }^{160}$. In coulomb explosion process, electrons from the sharp corners would be selectively excited and will be swiftly ejected ${ }^{147}$. Resulting expulsion will induce a positive ionic charge that explode to cause fragmentation. On the other hand, electrostatic explosion involves ions to be ejected along the static field generated by expelled electrons ${ }^{147}$. During both these mechanisms, it is believed that the energy absorbed is quickly equilibrated with time without causing whole particle melting or heat conversion. Based on this information, we hypothesize that cubic particles can selectively enhance light absorption at their LSPR modes and cause structural collapse without increasing the effective crystal temperature. In order to prove our hypothesis for shape assisted deformations in cubes, 
we designed $45 \mathrm{~nm}$ cubic nanoparticles and exposed them to ultrafast light pulses. For a more comprehensive understanding of LSPR modes and their influence on laser absorption we designed three different types of cubic nanoparticles having altered LSPR modes.

\subsection{METHODS AND CHARACTERIZATION}

\subsubsection{Materials}

Distilled water was used for all chemical experiments and synthesis. Silver nitrate, anhydrous ethylene glycol, sodium hydrosulfide, sodium hydroxide, polyvinyl pyrrolidone (PVP), hydrochloroauric acid, hydrochloric acid, nitric acid, ammonium hydroxide, titanium sulfate and silicon wafers were all purchased from Sigma-Aldrich. 4window fluorescent quartz cuvettes were purchased from Thor labs and Starna Cells. Nonlinear crystals (BBO crystal, type I SHG at $772 \mathrm{~nm}$; no coating; polished, thickness $0.75 \mathrm{~mm}$ ) were purchased from United Crystals Company.

\subsubsection{Instrumentation}

A Ti:Sapphire femtosecond laser system with regenerative power amplifier (Clark-MXR; model CPA-2110) was used for nanoparticle treatment. Two continuous-wave light sources (Avantes; models AvaLight-D-S-DUV and AvaLight-Hal-S) were used for covering incident spectral range from $185 \mathrm{~nm}$ to $2500 \mathrm{~nm}$. A Nonlinear crystal was used for second harmonic generation. An autocorrelator (Newport Corp.) was used for controlling pulse duration. Transmittance spectra was monitored using a 3 -channel UVIR high-resolution spectrometer (Avantes; model AvaSpec-3648-USB2-RM). AvaSoft 
software was used for transmittance analysis. FDTD Solutions (Lumerical Solutions Inc.) software was used to perform numerical simulations. A Headwall photonics imager equipped on a Cytoviva VNIR hyperspectral system was used for measuring hyperspectra. Joel TEM was used for imaging. FEI Tecnai F30 TEM with an EDX energy resolution of $134 \mathrm{eV}$ was used for elemental analysis. A Joel JEM-ARM200cF was used for STEMHAADF analysis with a resolution of $0.078 \mathrm{~nm}$. TEM images were analyzed on Gatan Digital Micrograph software. A Nanosurf easyScan 2 AFM was used for imaging. AFM images were analyzed using easyScan 2 software. A Malvern Nano-ZS was used for size distribution and zeta potential measurements. A Nanosight Nanoparticle tracking analysis (NTA) system was used for measuring particle concentrations. Metal concentrations were equalized using Inductively Coupled Plasma - Optical Emission Spectroscopy (ICP-OES) and Flame Atomic Absorption Spectroscopy (fAAS). UV-vis absorption was recorded using a Varian Cary Bio 50 UV-vis multi sampler spectrophotometer and a Cytation5 plate reader. The laser setup utilized an active damping SmartTable using pneumatic isolators by Newport Corp. A Newport stage with a controller was used for X-Y 96-well plate motion. Centrifugation was performed on a 5424 Eppendorf and refrigerated RC 6+ Sorvall centrifuge.

\subsubsection{Synthesis of cubic nanoparticles}

For this study 3 different types of cubic nanoparticles were prepared; [1] Nanocubes (NC), [2] Nanoboxes (NB) and [3] Nanocages (CG). Firstly, nanocubes were prepared using a polyol synthesis ${ }^{161}$. These nanocubes were used as a starting material for nanobox and nanocage conversions. Briefly, ethylene glycol was heated to $150{ }^{\circ} \mathrm{C}$ for 
$60 \mathrm{~min}$. Argon gas at $20 \mathrm{kPa}$ was introduced through a nozzle above the solution for 10 min. After initial argon flushing, the rest of the reaction remained under argon atmosphere $(10 \mathrm{kPa})$ at $155{ }^{\circ} \mathrm{C} .0 .7 \mathrm{~mL}$ of sodium hydrosulfide dissolved in ethylene glycol (3 mM) and $15 \mathrm{~mL}$ of polyvinylpyrrolidone (PVP) dissolved in ethylene glycol ( $20 \mathrm{mg} / \mathrm{mL}$; MW 55 000) were first degassed in Argon for 5 min followed by addition. After $8 \mathrm{~min}, 5 \mathrm{~mL}$ of degassed $\mathrm{AgNO}_{3}$ solution in ethylene glycol $(48 \mathrm{mg} / \mathrm{mL})$ was quickly added into the reaction solution. Reaction turned from a light yellow solution (at $1 \mathrm{~min}$ ) to green ochre (at $30 \mathrm{~min}$ ). The reaction was stopped at this moment and cooled in an ice bath for $5 \mathrm{~min}$ followed with addition of $40 \mathrm{ml}$ of acetone. The reaction mixture was centrifuged (7500 $\mathrm{g}$ for $40 \mathrm{~min})$ and the pellet was washed 4 times $(11000 \mathrm{~g}$ for $10 \mathrm{~min}$ ) with water to remove excess PVP. Finally, NC particles (40 mg yield) were resuspended in $10 \mathrm{ml}$ water.

In the next process $4 \mathrm{ml}$ of nanocubes were converted to nanoboxes via galvanic reduction with gold salt. Briefly, NCs were added to $10 \mathrm{ml}$ of PVP solution $(20 \mathrm{mg} / \mathrm{ml}$ in water) and heated to $100{ }^{\circ} \mathrm{C}$. This solution was then titrated with $17 \mathrm{ml}$ of $0.5 \mathrm{mM}$ $\mathrm{HAuCl}_{4}$ in a dropwise manner $(40 \mathrm{ml} / \mathrm{h})$ under constant stirring (400 RPM). The UVvisible spectrum of the nanoparticles was monitored continuously, and when peaks started to appear at $460 \mathrm{~nm}$ and $590 \mathrm{~nm}$, reaction was cooled on ice followed with addition of $200 \mu \mathrm{l}$ of $\mathrm{NH}_{4} \mathrm{OH}$. Once $\mathrm{NH}_{4} \mathrm{OH}$ dissolved the white $\mathrm{AgCl}$ precipitate, the particles were centrifuged (8000g for $40 \mathrm{~min}$ ). Resulting pellet was redispersed in PVP solution $\left(1 \mathrm{mg} / \mathrm{ml}\right.$ in water) and allowed to reflux at $100{ }^{\circ} \mathrm{C}$ for $10 \mathrm{~min}(400 \mathrm{RPM})$. Reaction was then cooled on ice and centrifuged (8000g for $40 \mathrm{~min}$ ) to obtain NB pellet. 
The pellet was further washed (11000g for $10 \mathrm{~min}$ ) with water and final NB pellet was resuspended in $4 \mathrm{ml}$ water.

Similar to NB synthesis, nanocages were prepared using nanocubes albeit further reduction until an $\mathrm{UV}$-Vis absorbance peak of $710 \mathrm{~nm}$ was attained. Final CG pellet was resuspended in $4 \mathrm{ml}$ water. If CG process was increased, nanoframes (NF; UV absorbance $>800 \mathrm{~nm}$ ) were found to form. All nanoparticulates solutions (NC, NB and CGs) were stored at room temperature under argon atmosphere.

\subsubsection{Characterization}

Cubic shapes and features of synthesized nanoparticles were imaged using TEM.

a

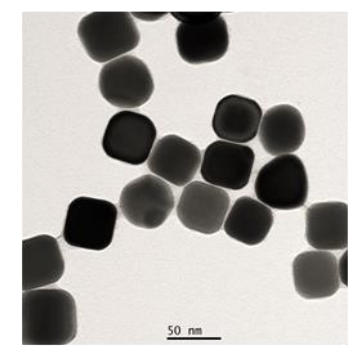

C

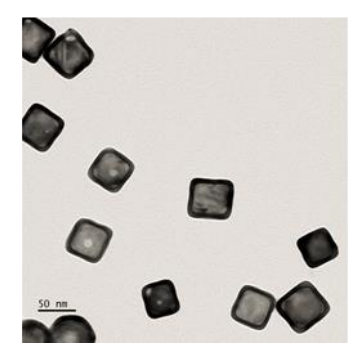

b

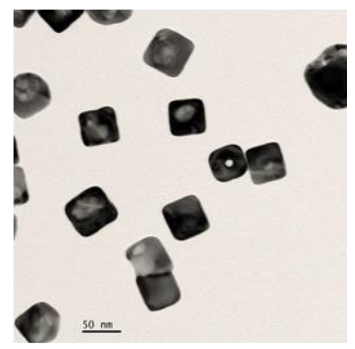

d

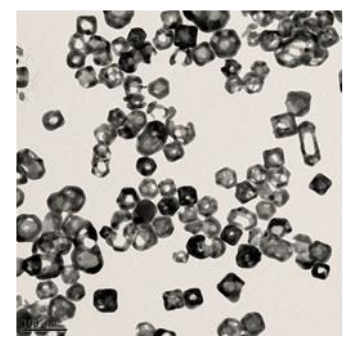

Figure 58. TEM images for (a) solid Ag nanocubes - NC, (b) Au-Ag nanoboxes with small cavity - NB, (c) hollow Au-Ag nanocages - CG and (d) Au nanoframes - NF. 
TEM images confirm the presence of solid nanocubes, nanoboxes with a small cavity, and hollow nanocages (Figure 58). Further reduction of CGs yielded nanoframe (NF) like structures and fragments (Figure 58). For cell viability, MTT assay was used. For elemental analysis, STEM-HAADF, STEM-EDS and EDX was used.

\subsubsection{Laser setup and real-time spectroscopy}

The femtosecond laser system (housed in a class 1000 clean room) had a fundamental wavelength of $772.00 \pm 1.15 \mathrm{~nm}$ with a full width at half maximum at $8.87 \pm 0.32 \mathrm{~nm}$ (Figure 59).

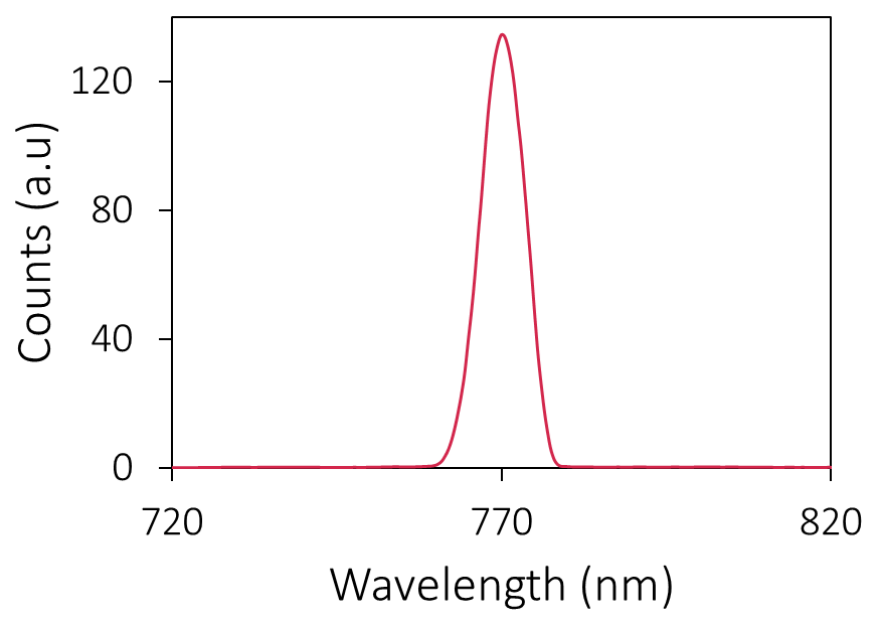

Figure 59. Fundamental wavelength of the Femtosecond pulse.

The system was set to produce a 150 fs pulse (1000 pulses/s) under linear polarization. For the setup, we allowed the laser beam to pass through a diaphragm, nonlinear crystal (to reduce wavelength to $386 \mathrm{~nm}$ ), a filter to block residual light and an optical attenuator (Figure 60 and 61). 


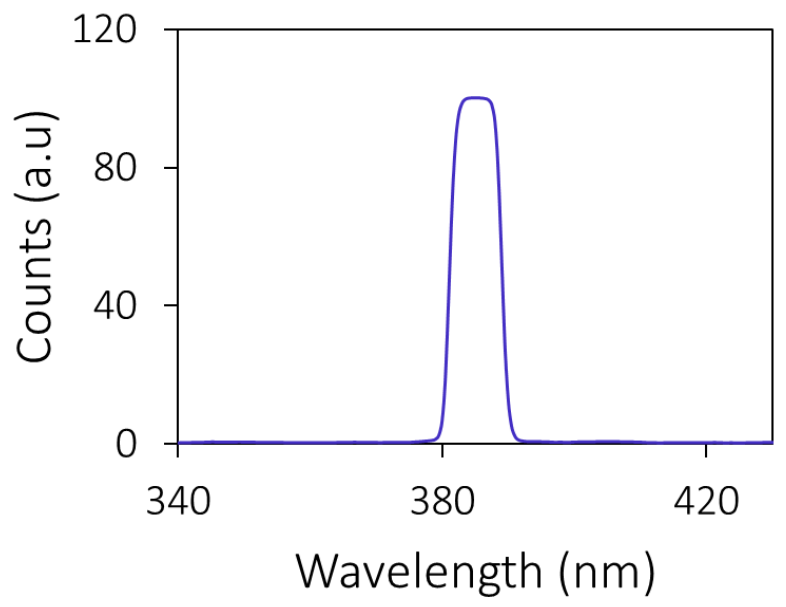

Figure 60. Reduced wavelength of the Femtosecond pulse at $386 \mathrm{~nm}$.

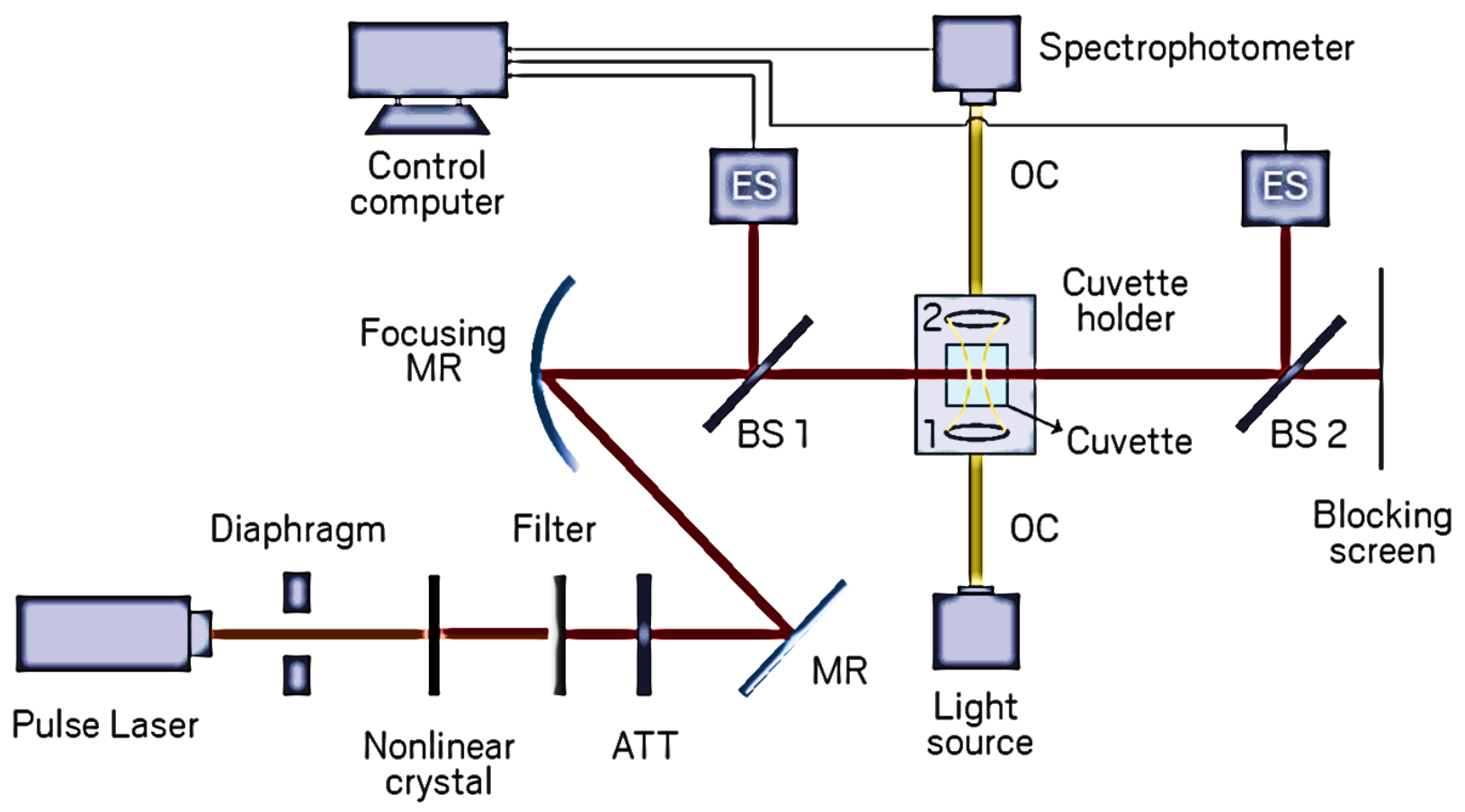

Figure 61. Custom laser setup for the experiments.

The beam was directed using bending mirrors to a focusing mirror $(\mathrm{f}=500 \mathrm{~mm})$. From the focusing mirror, the beam was directed through the sample cuvette. For the nonlinear crystal the beam was focused to the rear side of the cuvette. The beam was 
passed through 2 beam splitters both before and after the cuvette, for reflecting $11 \%$ of incident energy to 2 energy sensors. Finally beam path was ended using an absorbance screen. The spectroscopy unit was kept perpendicular to the beam direction through the sample cuvette. Light sources were delivered through an optical cable (OC) and collimated by a collimating lens unit 1 attached to the output (Figure 61). Light passing through the cuvette was collected by lens unit 2 attached to the input end of the OC and sent to the spectrophotometer. Signals from all units were processed using manufacturers software.

For CGs both incident beams of $772 \mathrm{~nm}$ and the second harmonic $386 \pm 0.85$ $\mathrm{nm}$ were used for exposure. For NBs and NCs, the $386 \mathrm{~nm}$ incident beam was used. An attenuator was used to vary the output $E_{\text {pulse }}$ (average energy of the pulse). $E_{\text {pulse }}$ was varied from $0.80 \mu \mathrm{J}$ to $135.0 \mu \mathrm{J}$ for both incident beam wavelengths. The standard deviation of pulse energy was estimated to be $0.8 \%$ at $772 \mathrm{~nm}$ and $2.0 \%$ at $386 \mathrm{~nm}$. The final diameter of the laser spot on the cuvette was measured to be $3.10 \pm 0.10 \mathrm{~mm}$. The amount of light transmitted by the two interfaces of the front cuvette wall was measured using sensors S1, S2 and S3 (Figure 62). The transmittance through an empty cuvette was measured to be $93 \%$ at wavelength $386 \mathrm{~nm}$, for the entire range of laser-pulse energy. The transmittance was measured to be $95 \%$ at low levels of pulse energy for water, methanol and butanol. In case of nanoparticle treatments, a beam splitter installed in front of the cuvette (Figure 61) reflected 6 to $9 \%$ of incident laser energy using a thin $(0.1 \mathrm{~mm}$ thickness) glass slide to the energy sensor. 


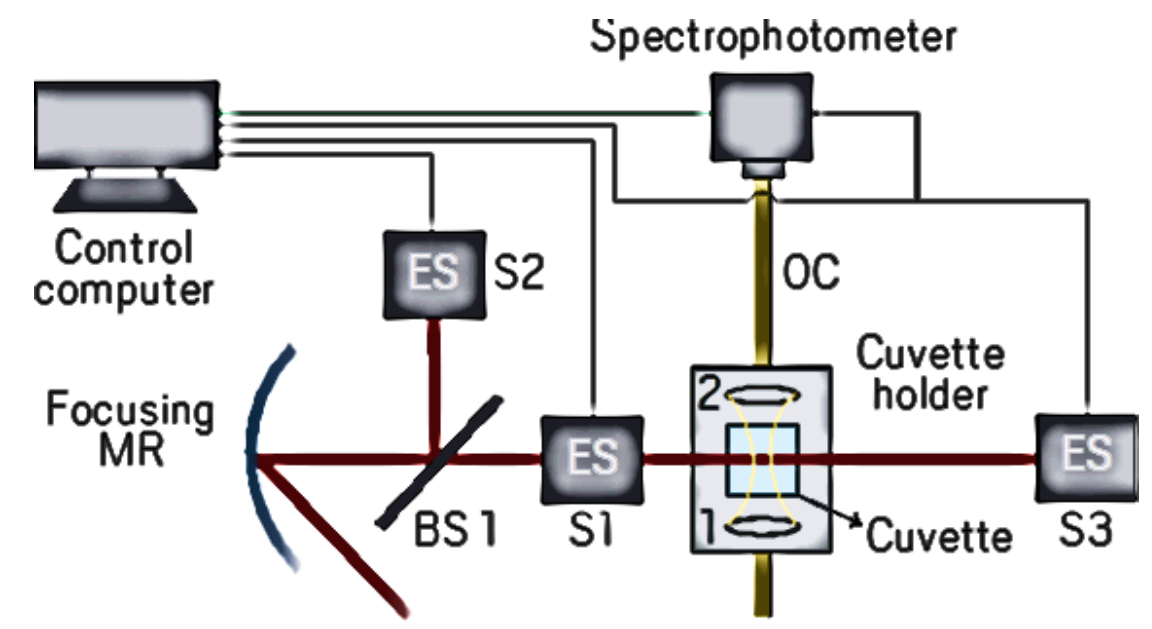

Figure 62. Sensors S1, S2 and S3 for measuring transmitted light within the setup.

Transmittance spectra for laser induced absorbance changes for nanoparticles was monitored through spectroscopy. The UV-IR high resolution spectrometer covered a range of wavelength from 185-2607 $\mathrm{nm}$ (Table 6). Resolution for ultraviolet and visible ranges of spectrum was measured to be $0.05 \mathrm{~nm}$ (full width at half maximum). Part of that range ( $185 \mathrm{~nm}$ to $1000 \mathrm{~nm}$ ) that contained the most prominent evidence of the modifications was selected for analysis. Subsequent analysis was processed on AvaSoft.

\begin{tabular}{lllll}
\hline $\begin{array}{l}\text { Channel } \\
\text { number }\end{array}$ & Wavelength range & Detector type & Integration time & $\begin{array}{l}\text { Number of spectra taken for } \\
\text { averaging }\end{array}$ \\
\hline $1(\mathrm{UV})$ & $185-756 \mathrm{~nm}$ & TCD1304 & $7.0 \mathrm{~ms}$ & 500 \\
\hline $2(\mathrm{VIS})$ & $596-1100 \mathrm{~nm}$ & TCD1304 & $3.7 \mathrm{~ms}$ & 1000 \\
\hline $3(\mathrm{NIR})$ & $937-2607 \mathrm{~nm}$ & Ham92xx & $3.9 \mathrm{~ms}$ & 1000 \\
\hline
\end{tabular}

Table 6. Transmittance spectroscopy: Parameters of the 3 channels. 


\subsubsection{Exposure of nanoparticles}

$1 \mathrm{ml}$ of nanoparticle solution $\left(\sim 4 \times 10^{10}\right.$ particles $\left./ \mathrm{ml}\right)$ was placed in a quartz cuvette made of UV-grade fused silica for exposure, while $1 \mathrm{ml}$ of solution was kept aside for control (no exposure). The height of the liquid for exposure and control were equally optimized for the meniscus to be above the laser spot. Samples were exposed to 10 trains of ultrashort laser pulses $(1$ train $=60000)$ with mixing in between each pulse train for maintaining sample homogeneity. Mixing was performed using an ultraclean sonicated glass tool. Transmittance spectra was measured before and after each train, using the spectra of control as reference signal for optimum sensitivity. This procedure allowed measurement of pure deviations from initial spectra for all samples. Solution temperature was monitored during exposure using a thermocouple attached to the bottom of the cuvette. Results show a very low variance of up to $3.75 \mathrm{~K}$ for all experiments. For each treatment, the spectra from solvent and empty clean cuvette was used as reference to negate influence of particle deposition on the walls during the exposure. Using the transmittance spectra, the shift of surface-plasmon resonance peak was acquired to plot shift of the resonance peak vs energy of laser pulses (at fixed pulse width and laser-spot diameter) (Figure 62). After each treatment, samples were placed in capped glass vials for analysis using UV-vis absorbance and NTA. Samples were separately kept in glass vials with argon gas blown on top prior to capping for further analysis. All cuvettes used for the exposure were cleaned using the following steps: (1) brief sonication in water, (2) detergent wash, (3) Aqua regia wash for 10 min, (4) Acetone/ethanol wash, (5) $\mathrm{NaOH}$ wash for $10 \mathrm{~min}$ and (6) repeated DI water wash followed by air drying for $1 \mathrm{~h}$. Cuvettes were then kept in sealed bags for experiments. 


\subsubsection{Cell culture}

PC3 human prostate carcinoma cells (ATCC, USA) were grown in RPMI 1640 medium (obtained from Gibco BRL). The media was supplemented with $4.5 \mathrm{~g} / \mathrm{L} \mathrm{d}$-glucose, 25 mM Hepes, $0.11 \mathrm{~g} / \mathrm{L}$ sodium pyruvate, $1.5 \mathrm{~g} / \mathrm{L}$ sodium bicarbonate, $2 \mathrm{mM}$ 1-glutamine, $10 \%$ heat-inactivated fetal bovine serum (Atlanta Biologicals, USA), and 1\% penicillin/streptomycin antibiotic solution. Cells were cultured in a humidified atmosphere of $95 \%$ air and $5 \% \mathrm{CO} 2$ at $37{ }^{\circ} \mathrm{C}$ (Thermo Scientific, USA).

\subsubsection{Cell viability tests}

In order to evaluate cell death due to laser exposure, ultrashort pulses were exposed to PC3 cells $\left(5 \times 10^{5}\right.$ cells $\left./ \mathrm{ml}\right)$ seeded in flat bottom 96 well plates both with and without nanoparticle treatment. For the exposure a new custom laser setup was made using a Newport stage controller (Figure 63). Range for the duration of exposure included the nanoparticle treatment durations. For these studies CGs were used for nanoparticle treatment $(3 \mathrm{~h})$. Influence of laser exposure, internalized particles during laser exposure was tested using MTT cytotoxicity assays. Results were plotted as energy dosage vs viability plots. Results show that the cell viability was not affected after laser treatment (Figure 64). Similarly, laser exposure for the same fluence and time used for all nanoparticle treatments did not adversely affect cell viability after CG internalization in PC3 cells (Figure 65). 


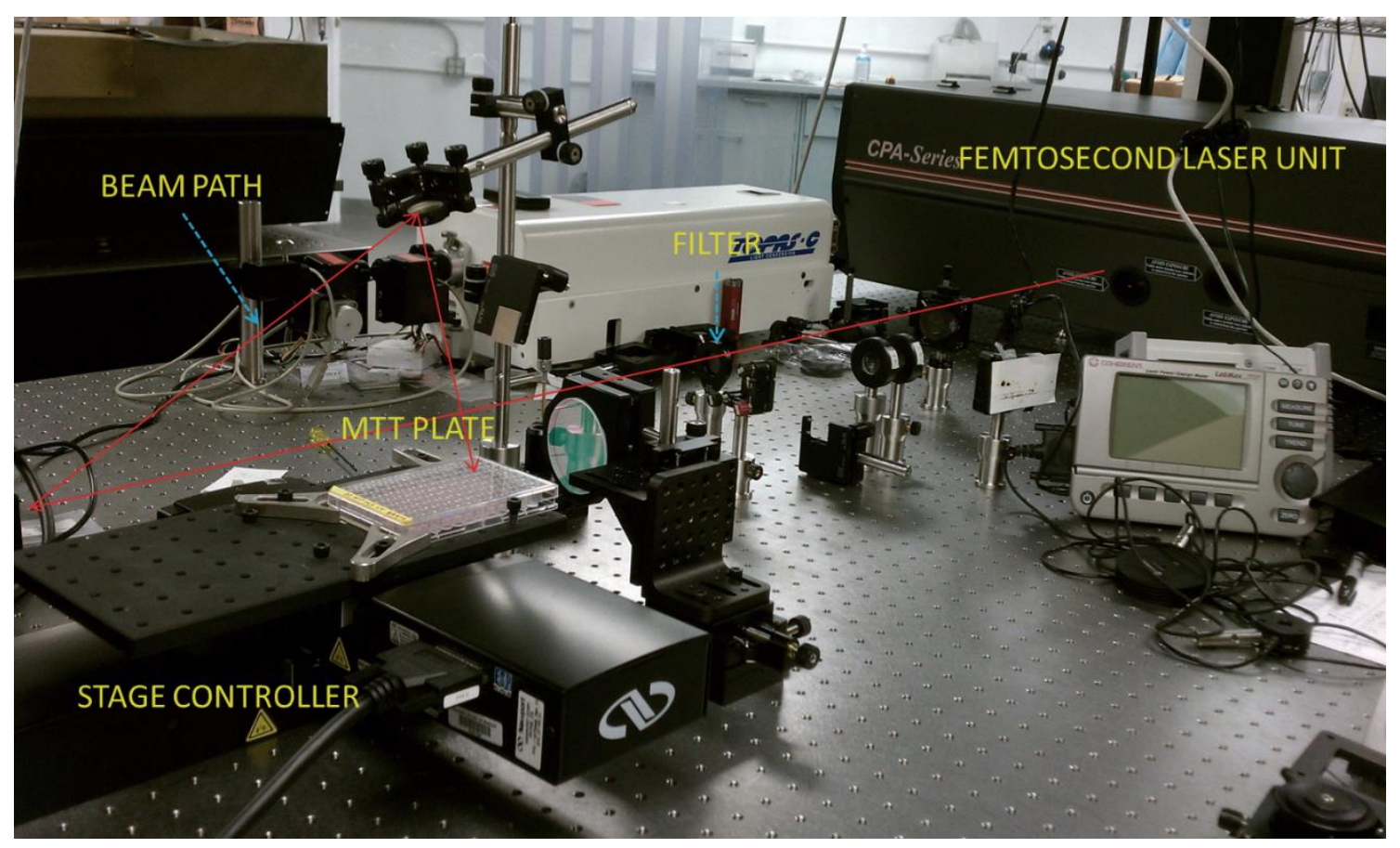

Figure 63. 96 well plates were exposed using a stage controller and focusing mirror.

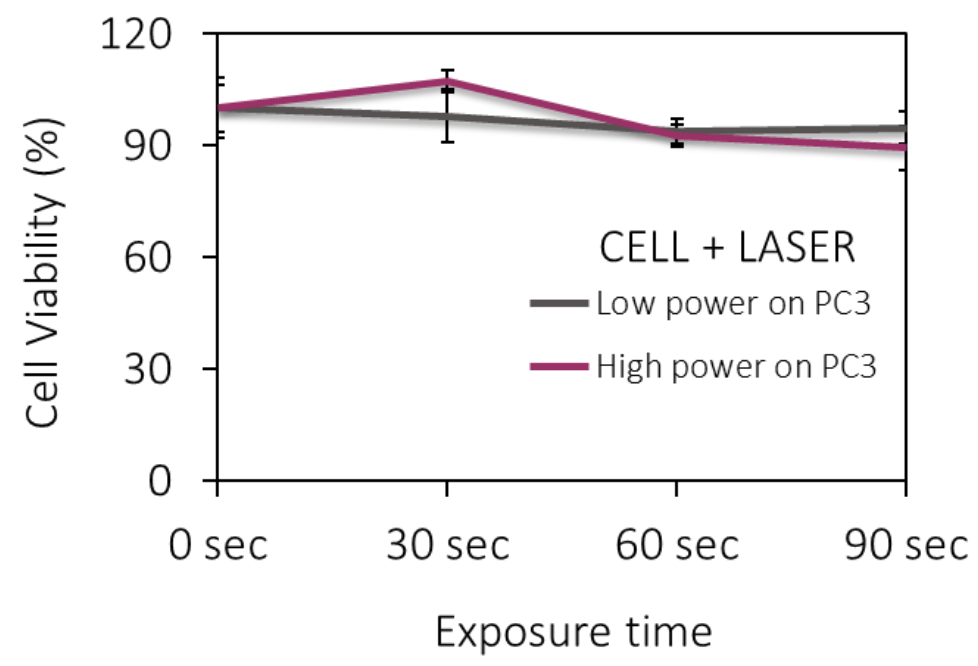

Figure 64. Femtosecond laser pulses at the NIR region did not affect cell viability. At the highest duration, both low and high power setting showed a viability of $85-90 \%$. Results are from triplicates. 


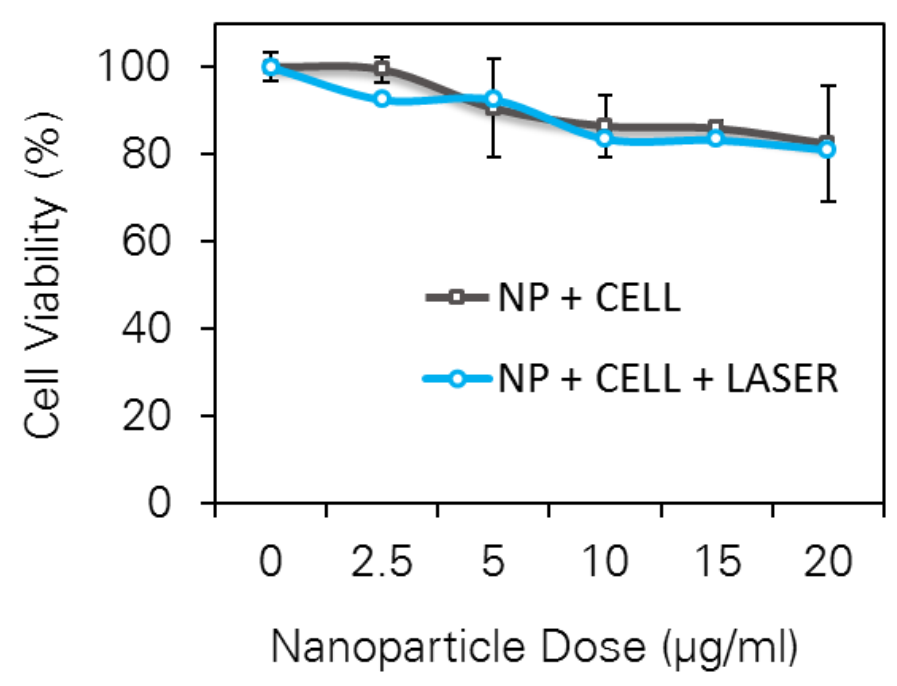

Figure 65. Femtosecond laser pulses at the NIR region did not affect cell viability after CG internalization. At the hig hest concentration, both low and high power setting showed a viability of 75-80\%. Results are from triplicates.

\subsubsection{FDTD simulation}

Numerical simulations were performed to solve electric field enhancements, power absorption and scattering properties of nanoparticles under a plane wave source. Nearfield E-field scattering, Electric field profiles and power absorbance was recorded for multiple cross sections of the simulated particle as well as for all 3 dimensions. Analyzed profiles were saved as 2-d images and 3-d plots. Simulations for a $100 \mathrm{fs}$ pulse was also recorded as a video for the simulated study.

For each simulation, wavelength-scale 3-d metal geometries were made in CAD. These geometries that included edge rounding, were based on TEM measurements for each type of particle. Interaction with light was solved using Maxwell equations using a conformal mesh (Cartesian). The simulation took Mie scattering in 3 dimensions into effect and compared the scattering and absorption cross sections. The problems were solved using a total-field scattered-field source (TFSF). Field profile $|\mathrm{E}|^{\wedge} 2$ in the YZ, 
a
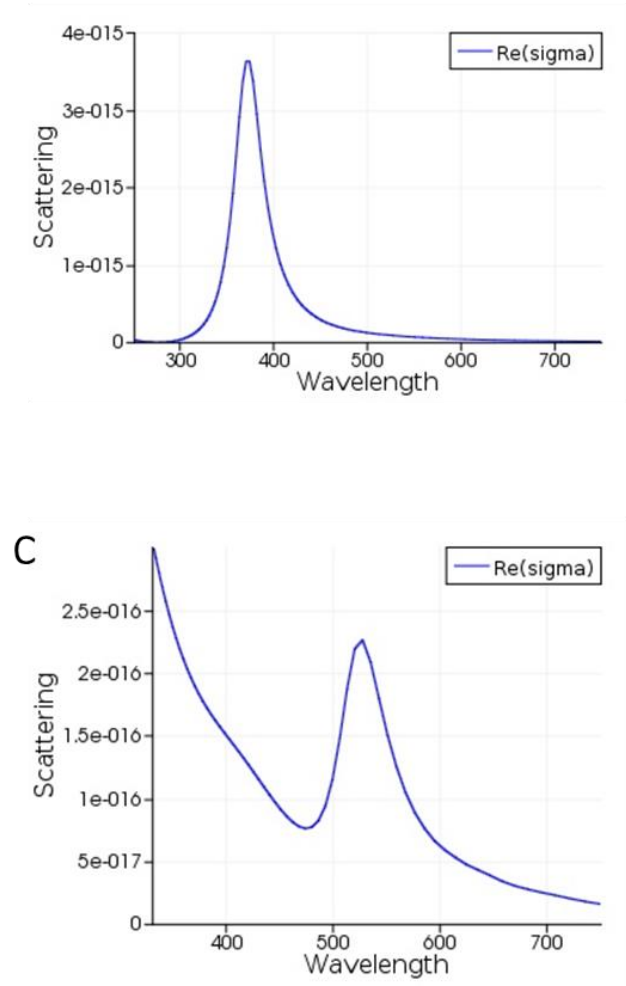

b
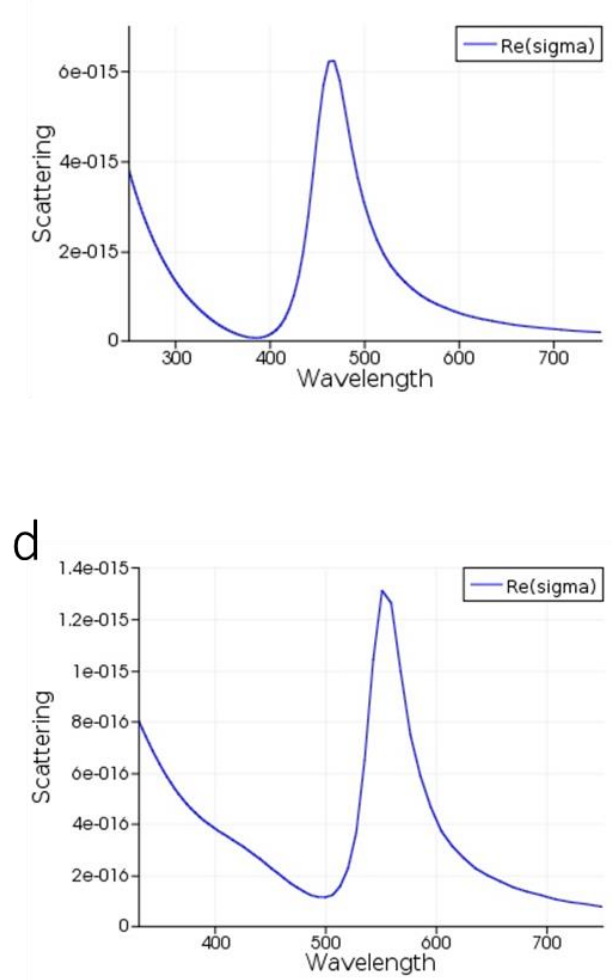

Figure 66. Scattering simulation for Ag and Au spheres. (a) $50 \mathrm{~nm} \mathrm{Ag} \mathrm{sphere,} \mathrm{(b)} \mathrm{Ag} \mathrm{sphere} \mathrm{with} \mathrm{a} \mathrm{dielectric} \mathrm{layer,} \mathrm{(c)} 50$ $\mathrm{nm}$ Au sphere, and (d) Au sphere with dielectric layer.

$\mathrm{XZ}$ and $\mathrm{XY}$ planes through the center of the particle were monitored during the simulation to calculate field enhancements. Spherical nanoparticle models were used as a control for the nanophotonic simulations. For the controls a $50 \mathrm{~nm}$ silver and gold sphere was used. Results for light scattering controls show accepted fit in model for both spherical particles based on earlier experiment (Figure 66) ${ }^{162,163}$. For the study, we also added a dielectric layer (to simulate PVP coating) on the surface. Results indicate a slight red-shift after introduction of a dielectric layer (Figure 66). Based on these results, we believe the experimental results would show a slight red shift relative to simulated models. 


\subsubsection{Single nanoparticle spectroscopy}

Samples after exposure were quickly diluted to $1 / 100$ th of the original concentration and sonicated for $1 \mathrm{~min} .2 \mathrm{ul}$ of this diluted solution was then placed on an ultrasonically cleaned glass slide (Nexterion Glass B laser cut slides; Schott). Drop was spread using a Nexterion Glass B coverslip and the edges were tightly sealed. Samples were then placed in a hot air oven $\left(45^{\circ} \mathrm{C}\right.$ ) for $10 \mathrm{~min}$ to air-dry. Slides were fixed on a 100x (oil immersion) objective and particles were focused using a dark field back-scattering scope combined with a $500 \mathrm{~nm}$ long pass filter to eliminate harmonics. A VNIR hyperspectral imaging system with a spectral resolution of $2.8 \mathrm{~nm}$ was then used to measure nanoparticle spectra from single illuminated nanoparticles using an incident

light source (aluminum bulb with a 400-950 nm range). For each sample, 10 nanoparticle spectra were recorded and a hyperspectral library was created using ITT ENVI software.

\subsubsection{HR-TEM STEM and EDX analysis}

Samples were prepared by dip coating in solution and air-dried for $15 \mathrm{~min}$. STEM-HAADF images were produced in dark field mode obtained by beam tilting $(200 \mathrm{kV})$. Results show that NCs are highly crystalline due to growth of a uniform silver crystal seed (Figure 67). 


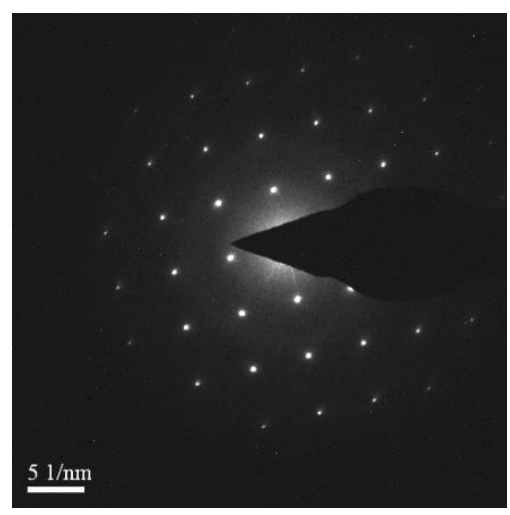

Figure 67. Crystallography for solid Ag nanocubes indicates a crystalline structure.

However, NBs and CGs are polycrystalline due to formation of silver-gold alloy during synthesis (Figure 68). Particles exposed at varying levels of energy were imaged. Particles at spectral threshold $(<20 \mu \mathrm{J})$ did not shown any changes, while particles exposed at deformation threshold $(<80 \mu \mathrm{J})$ showed selective fragmentation at sharp curvature points as predicted by simulations and LSPR modes of the respective nanoparticle structure. Their final structure was polycrystalline as well. (Figure 69).

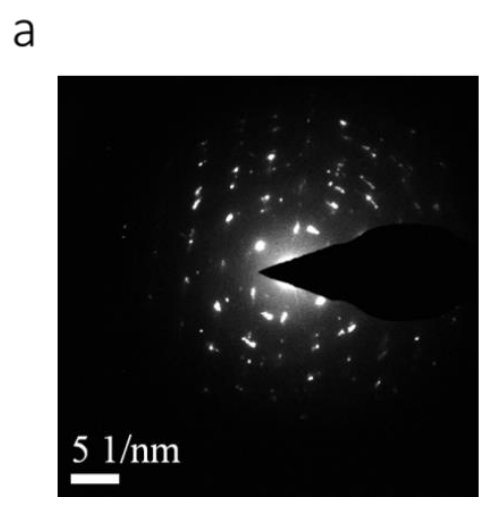

b

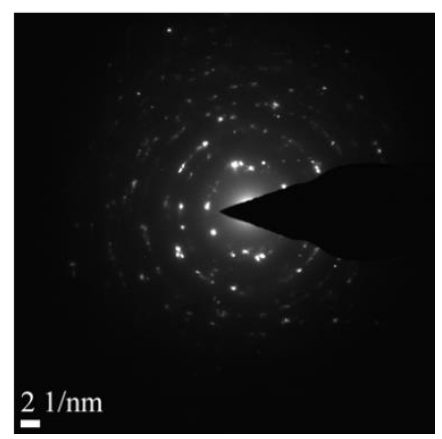

Figure 68. Crystallography for porous particles: (a) nanoboxes and (b) nanocages, indicate a polycrystalline structure. 


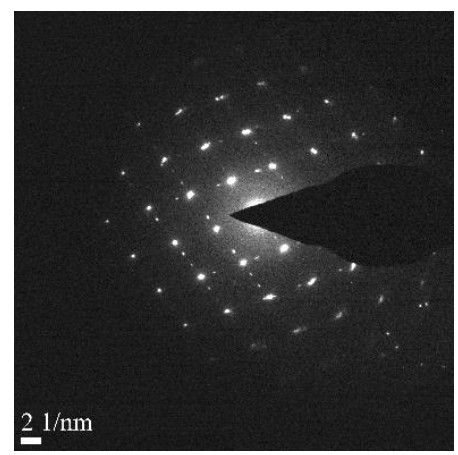

Figure 69. Crystallography for fragmented particles indicate a polycrystalline structure.

The rest of the structure remained largely intact without any structural modifications. In fact, the polycrystalline nature of NBs and CGs were preserved after exposure. On the other hand, all particles were seen to have crystalline structure at very high incident energy exposure $(>90 \mu \mathrm{J})$ signifying particle melting (Figure 70).

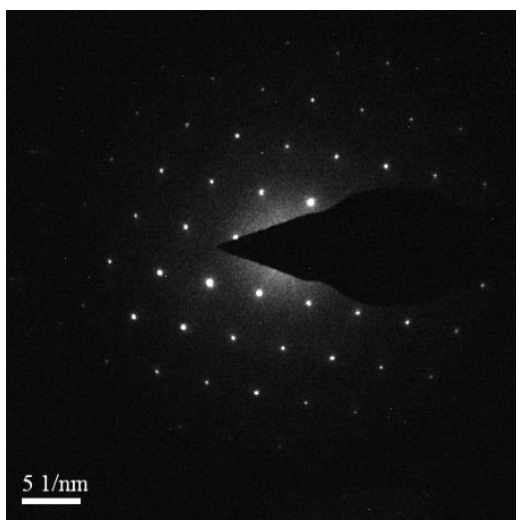

Figure 70. Crystallography for melted spheres indicate a crystalline structure.

At melting threshold all particles formed spheres. STEM-EDS revealed a uniform alloyed composition in NB and CG after exposure (Figure 71). 


\section{a}

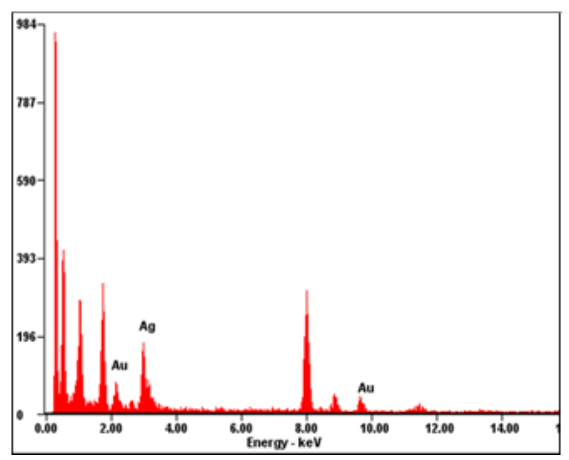

b

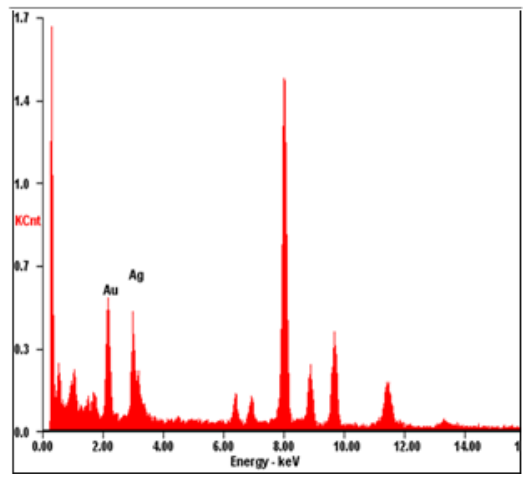

Figure 71. Au-Ag alloy composition for (a) Nanoboxes and (b) Nanocages. Results indicate the crystal structure remains preserved after laser treatment.

\subsubsection{Atomic force microscopy}

Nanoparticle samples diluted to $1 / 10^{\text {th }}$ were drop casted over a clean silicon wafer. Samples were then spin coated (1000 RPM) to form a clean monolayer on the wafer. The wafer was then placed under the AFM cantilever and imaged using non-contact mode at $10 \mathrm{~V}$ in $5 \mathrm{mV}$ steps.

\subsubsection{Size distribution analysis}

$500 \mu 1$ of sample solution before and after treatment was placed in clean size analysis cuvette and analyzed using dynamic light scattering. Histograms for effective hydrodynamic size in specific solvents were recorded and size vs intensity curves were plotted using zetasizer software. 


\subsubsection{Image analysis using TEM}

$8 \mu \mathrm{l}$ of nanoparticle suspension in water was air dried for $15 \mathrm{~min}$ in a hot air oven $\left(40^{\circ} \mathrm{C}\right)$ over a CF300-Cu carbon film based copper grid. For suspensions in other solvents, grids were placed on clean glass substrates and the sample was drop $(2 \mu 1)$ casted 5 times. This was followed by drying for $5 \mathrm{~min}$ in hot air oven $\left(40^{\circ} \mathrm{C}\right)$. Grids were then imaged at $120 \mathrm{kV}$ and images were analyzed using ImageJ.

\subsubsection{Statistics}

All statistics were performed using One-way Anova on Minitab. Average values from triplicates or more were calculated and used for statistical analysis.

\subsection{RESULTS AND DISCUSSIONS}

\subsubsection{Properties of different nanocubic architectures}

For this study, we fabricated three different nanoparticles with cubic shape having distinctive morphologies. These morphologies allow change in LSPR modes of the nanoparticle and their subsequent interactions with incident light. The cubic particles (45 $\mathrm{nm}$ edge length) were categorized into two groups namely solids and hollow particles. Nanocubes (NC) represented the solid group, while Nanoboxes (NB) and Nanocages (CG) represented the hollow group.

By having cavities within the solid surface we intended for formation of inner LSPR modes in contrast to exterior LSPR modes displayed by solid cubes. The sizing of these cavities between NB and CG, allowed us to investigate the interplay between exterior and interior modes and their effect on light absorption efficiency. NCs had 
sharp corners that can enhance EMF on the extreme convex curvatures of the particle. In contrast, NBs had a combination of sharp corners and a small central cavity that can enhance EMF both on the extreme convex (exterior) and extreme concave (interior) curvatures of the particle. On the other hand, CGs had sharp corners with a large cubic cavity to produce extremely thin surfaces on its faces ( $\sim 7 \mathrm{~nm}$ thickness). Thin films can allow intense absorption to stimulate plasmonic light traps. We expected these characteristic structures to give rise to dissimilar LSPR signatures. This will affect the light absorption characteristics in the visible to NIR range.
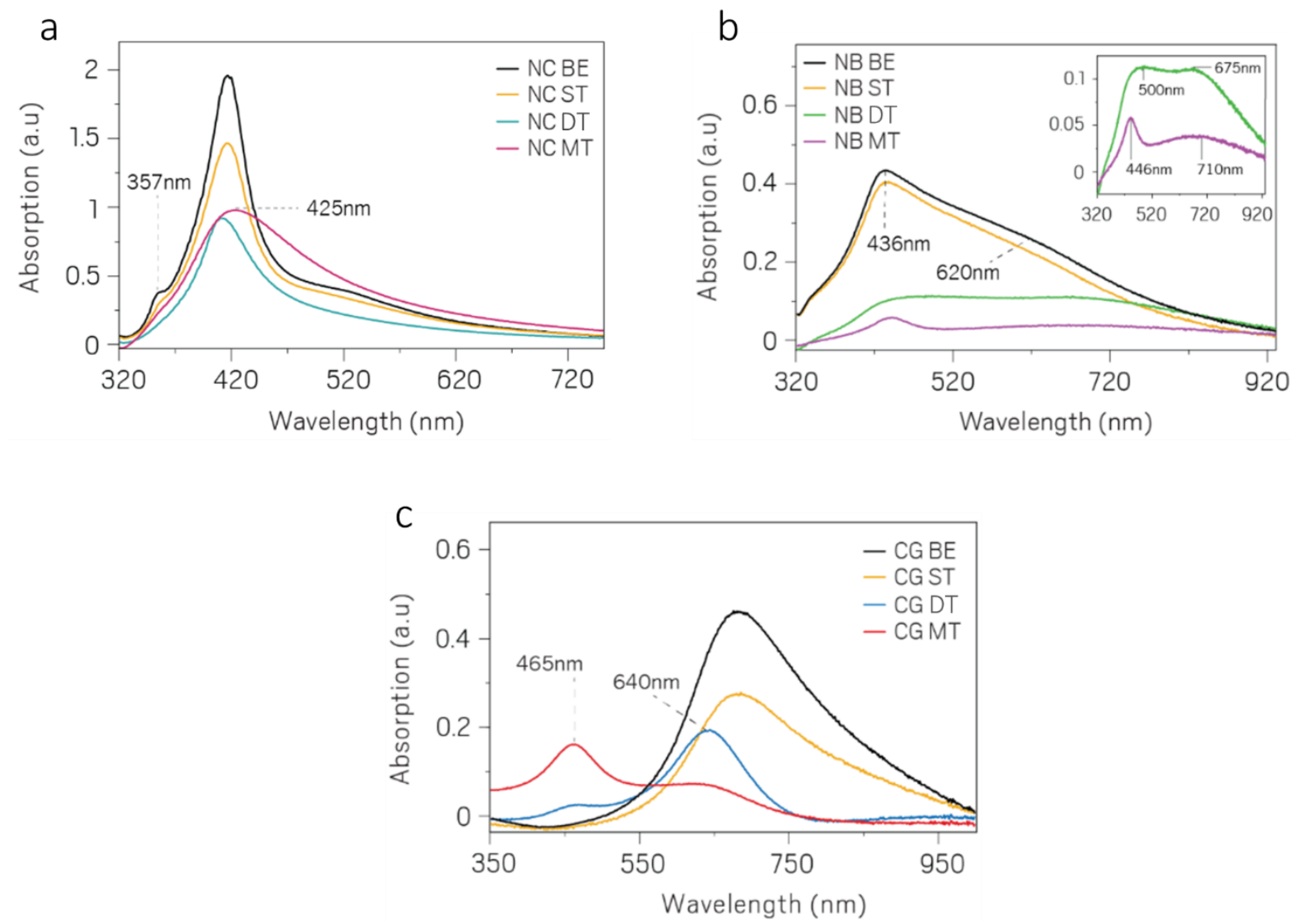

Figure 72. UV-vis absorbance profiles for (a) Nanocubes (b) Nanoboxes and (c) Nanocages, before and after exposure. BE, ST, DT and MT stand for Before exposure, Spectral threshold, Deformation threshold and Melting threshold respectively. 
Indeed, results from UV-Vis absorbance show that these particles before exposure (BE) had different absorbance maxima. NC had 2 peaks at 357 and $410 \mathrm{~nm}$ (Figure 72). The intense $410 \mathrm{~nm}$ peak represented the total particle absorption while it has been previously shown that the $357 \mathrm{~nm}$ peak corresponded to the sharp corners ${ }^{123}$. NB as predicted, had two intense peaks at $436 \mathrm{~nm}$ and $620 \mathrm{~nm}$ owing to inner and outer curvatures (Figure 72). CG had a single relatively broad intense band at $710 \mathrm{~nm}$ indicating an overlap of plasmons from the sharp corners and thin faces (Figure 72). Based on these results, we decided to tune the wavelength of ultrashort light puls es to match the absorbance peaks, allowing highly localized plasmonic resonance to occur.

\subsubsection{Simulating nanoplasmonic field enhancements}

To study the physical nature of nanoplasmonic behavior in the 3 different cubic architectures, we solved for electromagnetic field enhancements using numerical simulations. By solving, we investigated the nanophotonic interaction both within the particle and its surface. In order to negate any errors, we first simulated scattering spectra that is a function of LSPR. Results from scattering spectra models indeed fit our experimental UV-Vis absorbance spectral characteristics that is also a function of LSPR. As a next step we monitored the electric fields of the nanoparticle. The nearfield E-field scattering for all three particles showed a drastic enhancement at corners (Figure 73). Remarkably, results for total E-field confirm that sharp features such as corners and cavities, definitely enhance electromagnetic fields relative to the particle (Figure 74). Sharp corners in NC and CG were seen to enhance by 7 and 10 folds, while NB had an average enhancement of 6 folds. 
a

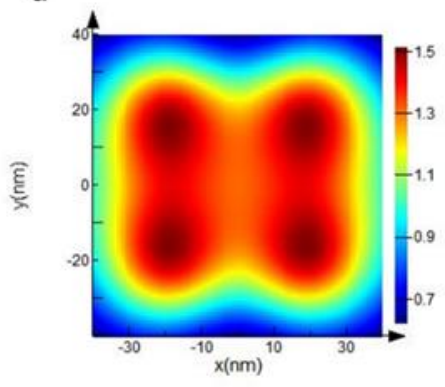

b

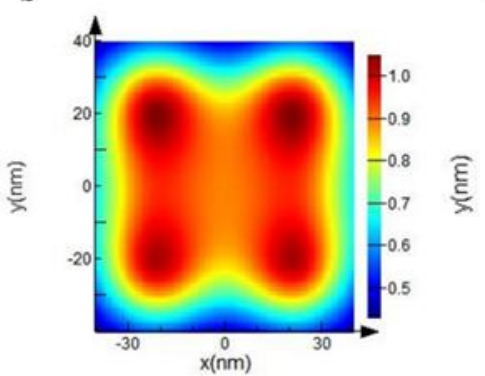

c

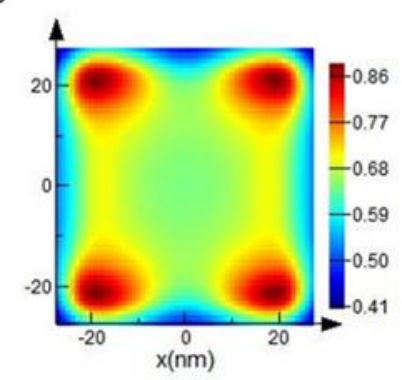

Figure 73. Simulated near-field E-field scattering for (a) Nanocubes (b) Nanoboxes and (c) Nanocages.

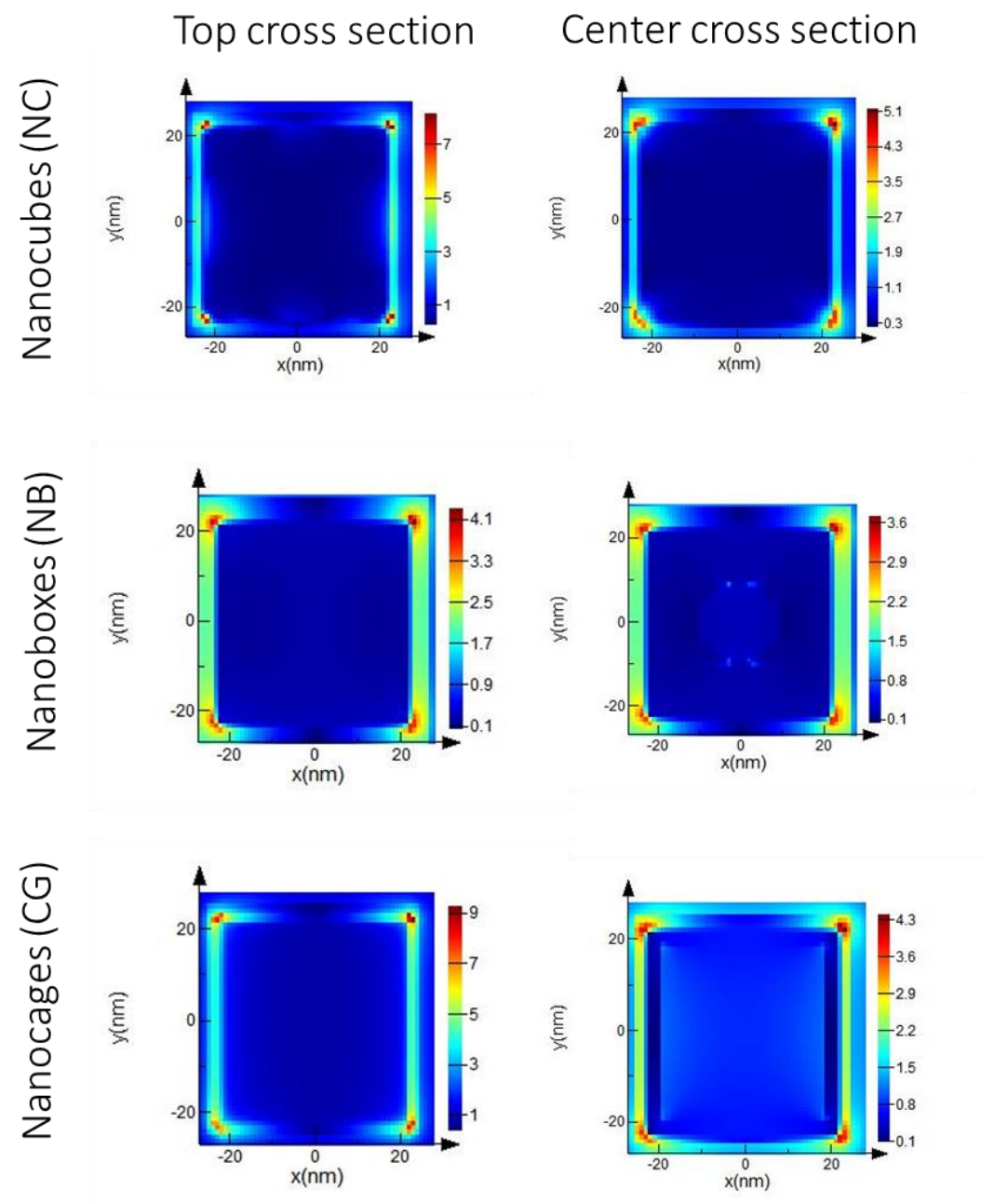

Figure 74. Simulated total E-field for (a) Nanocubes (b) Nanoboxes and (c) Nanocages for top and center cross sections for each particle structure. 


\section{Top cross section Center cross section}
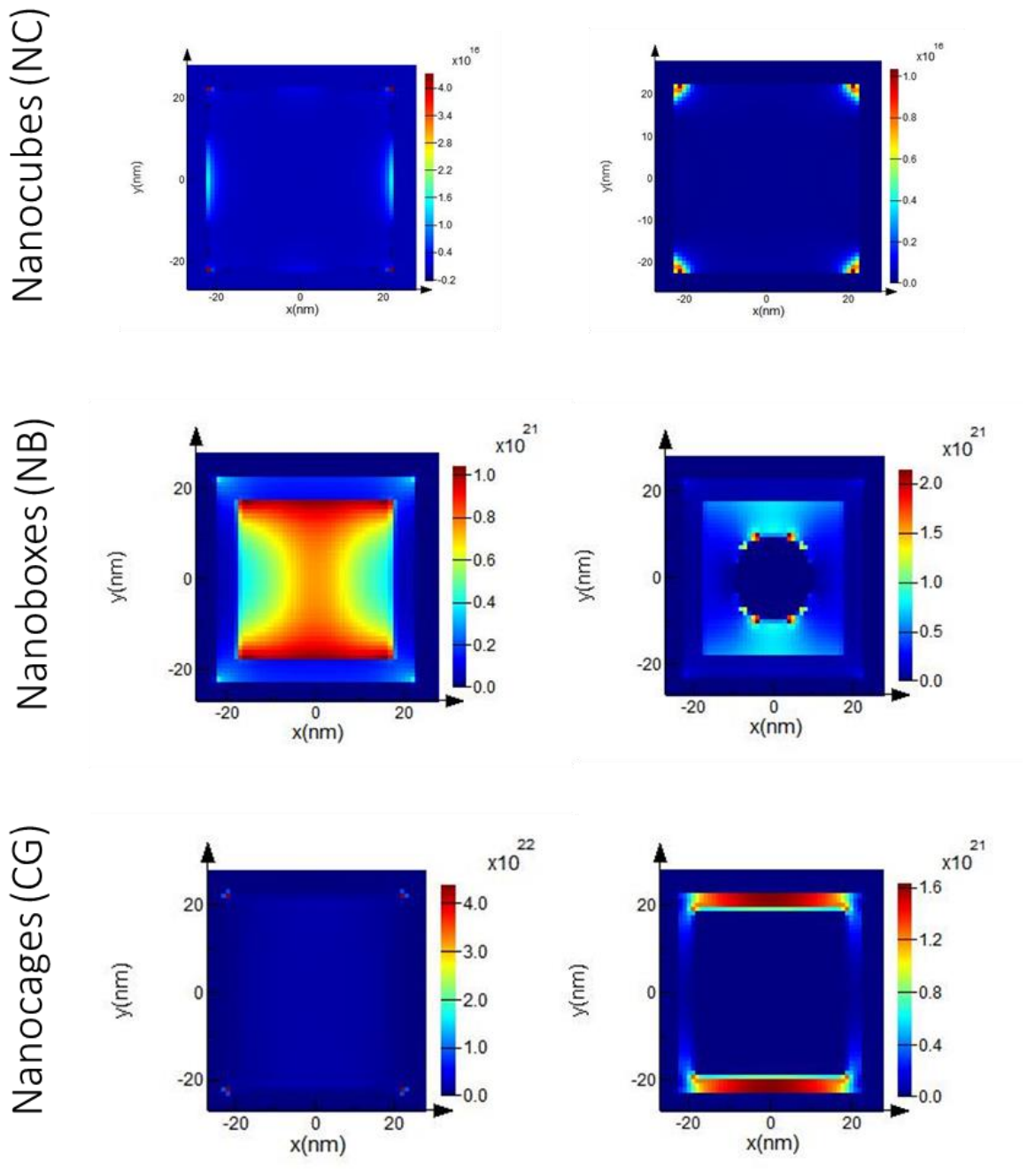

Figure 75. Simulated power absorbance profiles for (a) Nanocubes (b) Nanoboxes and (c) Nanocages for top and center cross sections for each particle structure.

Additionally, NB cavities showed an enhancement of 4 and 5 folds for increasing cavity diameters respectively. This dip seen in NB corner enhancement factors could be due to the LSPR coupling with cavities. These localized enhancements were considered as active plasmonic hot-spots. On the other hand, simulations from spheres indicated a very low enhancement of 2 fold as compared to high aspect ratio structures. 
As a next step we decided to monitor the power absorbance of light through the material. Data for all 3 cubic structures suggest an average absorbance at a factor of $10^{21}$. Interestingly, NC and CG did not show any extreme absorbance at the nanoparticle core, with one being a solid and one having a hollow structure (Figure 75). However, NB absorbance patterns indicated increased power absorbance at the cavities (Figure 75).
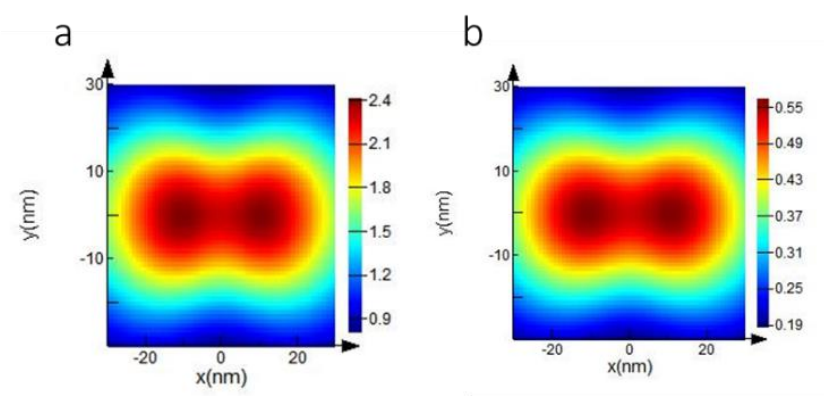

Figure 76. Simulated near-field E-field scattering for (a) silver spheres and (b) gold spheres.

This anomalous material behavior signifies an active LSPR coupling between the exterior and interior LSPR modes. This field coupling confirms the reason for the field enhancement dip at the corners in NBs. Based on these results we hypothesize that cubic particle would have intense absorbance specifically at corners. This would permit highly localized modifications at the corners. Furthermore, we also anticipated that the corners in NB would not play a major role in modification due to energy interference with cavities. Taken together, the simulations demonstrate high E-field enhancements owing to the sharp convex (corners) and concave (cavities) curvatures. This enhancement helps sub-wavelength localization of light, and therefore can theoretically 


\section{Top cross section Center cross section}
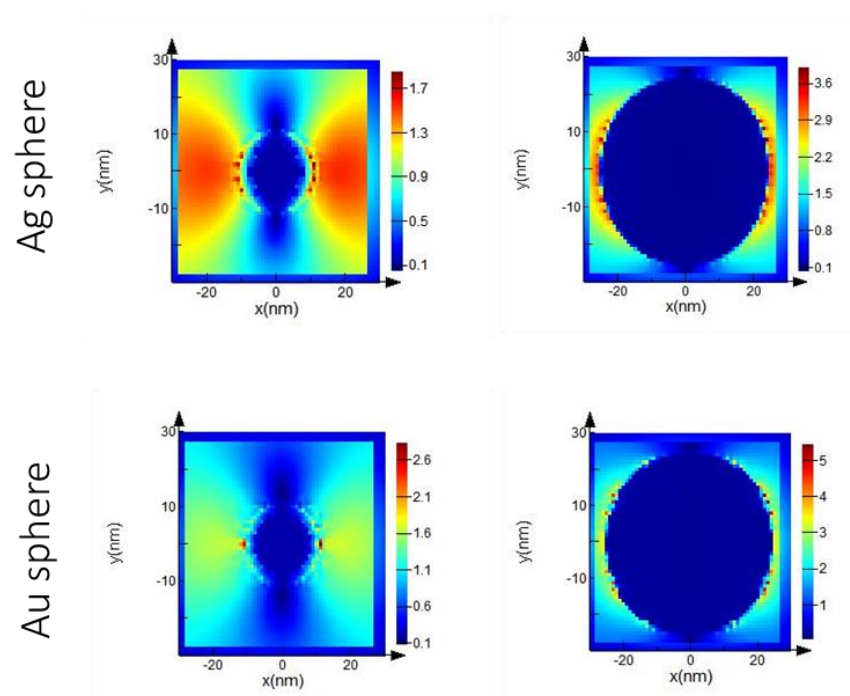

Figure 77. Simulated total E-field for (a) silver spheres and (b) gold spheres for top and center cross sections for each particle structure.

Top cross section Center cross section
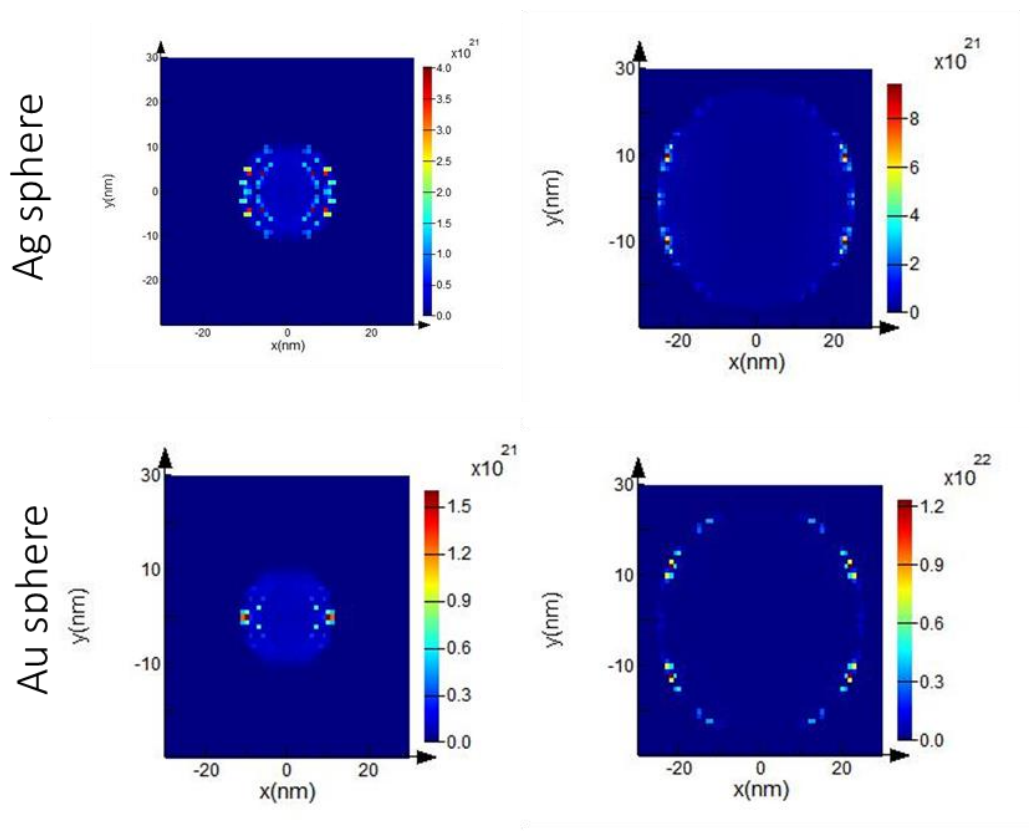

Figure 78. Simulated power absorbance profiles for (a) silver spheres and (b) gold spheres for top and center cross sections for each particle structure. 
reduce the amount of energy needed to modify a structure. While spherical particles also have near-field scattering properties, the total E-field enhancements are lower by 2-4 folds due to absence of shape anisotropy (Figure 76 and 77). In fact, spherical particles lack power absorbance properties and therefore are inefficient in absorbing energy (Figure 78). This result implies spheres require higher energies to work with, that proves the suitability of high aspect ratio structures in controlled release applications. However, these results indicate a theoretical possibility of low power light-induced modifications. In order to experimentally prove we exposed the nanoparticles to femtosecond laser pulse and studied ensuing modifications.

\subsubsection{Activation thresholds of cubic nanoparticles}

To excite the nanoparticles using an external energy source, we used a linearly polarized 100-150 fs ultrashort pulse with a $1 \mathrm{~ms}$ time gap. This time gap assures nanoparticle relaxation and temperature equilibrium in between every pulse. For preliminary studies, we steadily increased energies and measured the amount of energy needed for spectral changes, that is a function of shape and structure (Equations 1 and 2). Subsequently at each step of spectral change the nanoparticles were characterized for modifications using single nanoparticle spectroscopy and TEM. Spectroscopically, we identified three unique transmittance spectra deviations that were statistically significant ( $\mathrm{p}<0.001$ ) (Figure 79). These unique spectral changes (blue/ red shifts) take place at three different incident energies. Even when the laser polarization was configured to circular, similar spectral variations were observed. This indicates the changes to be irrespective of electric-field direction (Figure 79). These results confirm 
the presence of discrete energy levels (activation thresholds) required for spectral changes. The average activation thresholds for NCs were measured to be $\sim 14 \mu \mathrm{J}, \sim 70$ $\mu \mathrm{J}$ and $\sim 130 \mu \mathrm{J}$ respectively, For NBs these thresholds were $\sim 11 \mu \mathrm{J}, \sim 57 \mu \mathrm{J}$ and $\sim 125$ $\mu \mathrm{J}$ respectively. While for CGs these thresholds were $\sim 9 \mu \mathrm{J}, \sim 41 \mu \mathrm{J}$ and $\sim 121 \mu \mathrm{J}$ respectively. The spectral changes at 9-14 $\mu \mathrm{J}$ did not significantly shift the curves, however, the higher energy thresholds $(>20 \mu \mathrm{J})$ showed drastic SPR shifts. These significant activation thresholds indicate quantized interactions. Specifically, the data signifies that around $\sim 40 \mu \mathrm{J}$ the particles start to experience changes to their structure that is function of LSPR. To further understand the relation between the spectral changes and particle structure we studied the modifications produced at these energy levels.

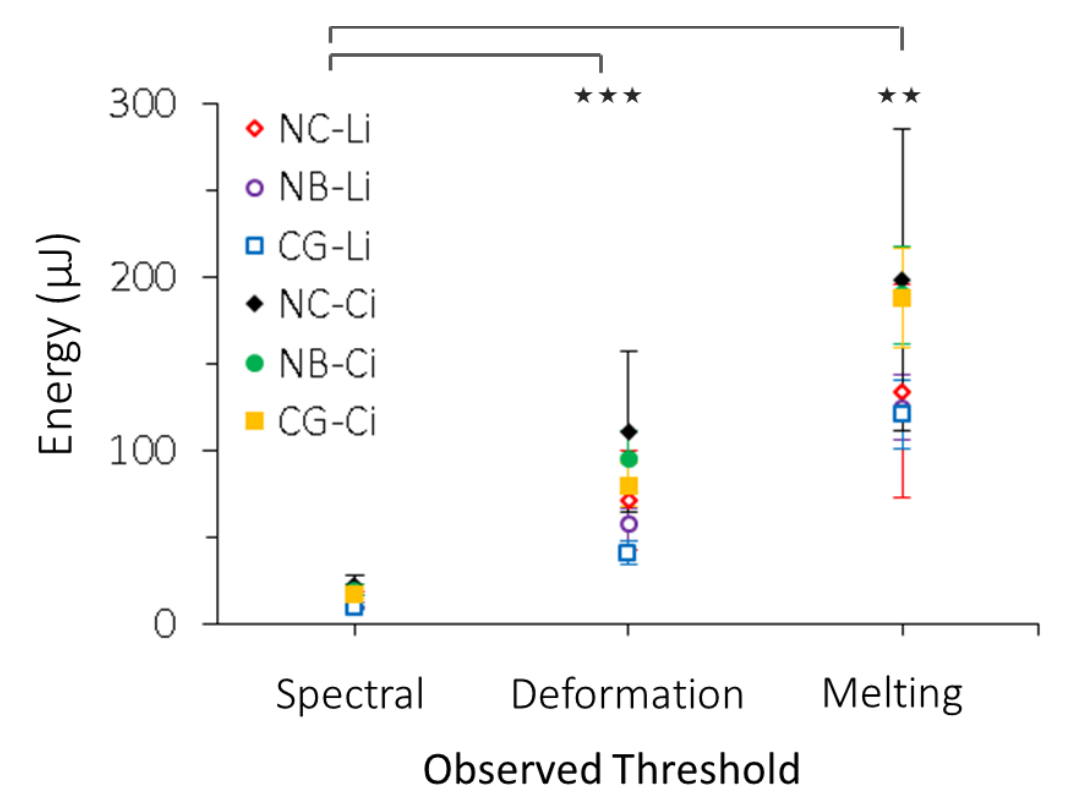

Figure 79. Activation thresholds for (a) Nanocubes (NC) (b) Nanoboxes (NB) and (c) Nanocages (CG) using linear (Li) and circular $(\mathrm{Ci})$ polarization exposure by femtosecond light pulses. Results indicate discrete levels of interaction energies for the three particles. ${ }^{\star} \mathrm{p} \leq 0.01$, and ${ }^{\star \star \star} \mathrm{p} \leq 0.001$ and the values were analyzed using one-way anova. 


\subsubsection{Controlled particle modifications}

Particle modification after laser treatment were further investigated by TEM and characterized by UV absorbance spectroscopy, size distribution analysis and single nanoparticle spectroscopy. As a control study, nanoparticles were treated at wavelengths that did not match its absorbance peaks. TEM images of the control samples showed no particle modifications. This indicates that nanoparticles with fixed absorbance properties can be laser treated with high specificity (Figure 80). As a next step, particle modification was studied after laser wavelength matching. In the corresponding 3 activation thresholds, no structural modifications were observed at the

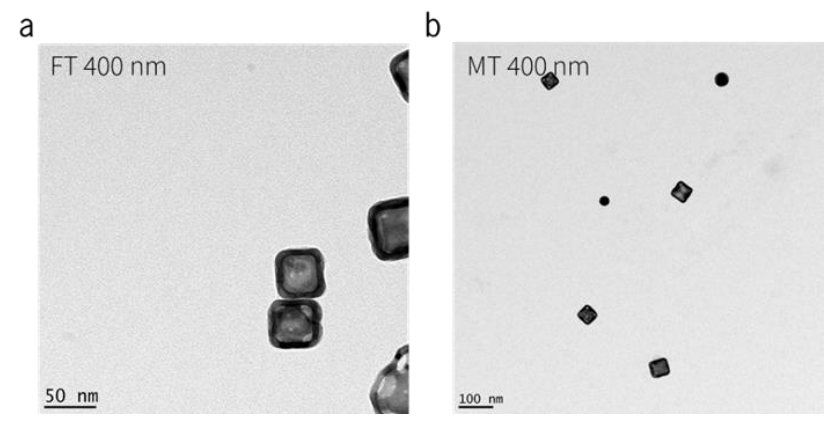

Figure 80. No particles modifications were observed in CGs $(710 \mathrm{~nm})$ when the laser wavelength was detuned to $400 \mathrm{~nm}$ at both (a) medium; 20-80 $\mu \mathrm{J}$ and (b) high power; (> $90 \mu \mathrm{J})$.

lowest energies (ST) (Figure 81). TEM images at tuned wavelengths with energies less than $20 \mu \mathrm{J}$ did not show any structural modification, implying the role of surface molecules rather than the structure, in LSPR shift. It is known in literature, that along with structure and shape, surface properties also serve as function of LSPR ${ }^{164}$. 
a

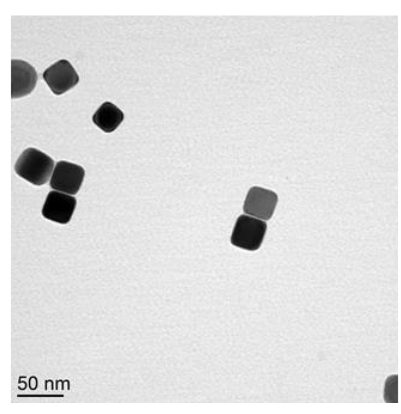

b

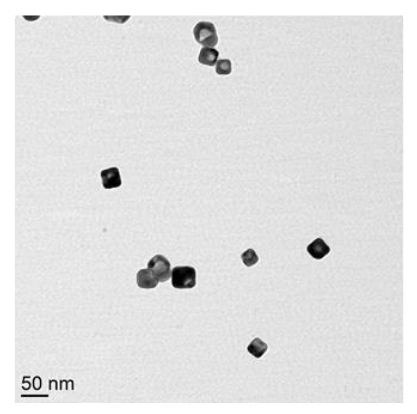

C

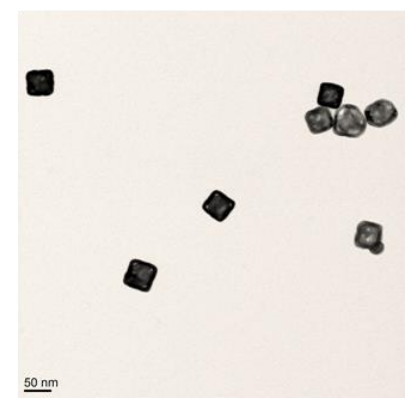

Figure 81. No particles modifications were observed in (a) Nanocubes, (b) Nanoboxes and (c) Nanocages at spectral threshold (ST).

Therefore, electron heating at the surface might generate enough kinetic energy to heat the skin layer $\left(l_{\mathrm{s}}\right)$ and transfer heat to surface molecules ${ }^{147}$. This transfer of energy to surface polymers (for example, PVP) on the surface, effect absorption and refractive index properties. This interdependence of LSPR to surface molecules have been previously documented in many studies, and is applied in SPR based sensors ${ }^{132,139,162 .}$

Contrastingly, particles modifications were observed in both the high energy thresholds. At the medium threshold $(20-80 \mu \mathrm{J})$ modifications at sharp curvatures were found, while at the highest threshold (>90 $\mu \mathrm{J}$ ) particle melting was observed (Figure 82). Based on these results we classified the 3 thresholds as [1] spectral threshold (ST; only spectral shift), [2] deformation threshold (DT, lowest energy required for localized fragmentation) and [3] melting threshold (MT, lowest energy required for total melting). TEM results for NCs at DT show blunted corners, with the rest of particle structure intact. At MT, NCs undergo a melting transition to form spheres as they have lowest surface area to volume ratio and therefore need the lowest energy to form. Similarly, CGs that have thin faces, fragmented at the corners in DT, while formed spheres at MT (Figure 82). NBs also formed spheres at MT, however the corners were not blunted at DT. Instead the concave curvature expanded to enlarge the cavity. 

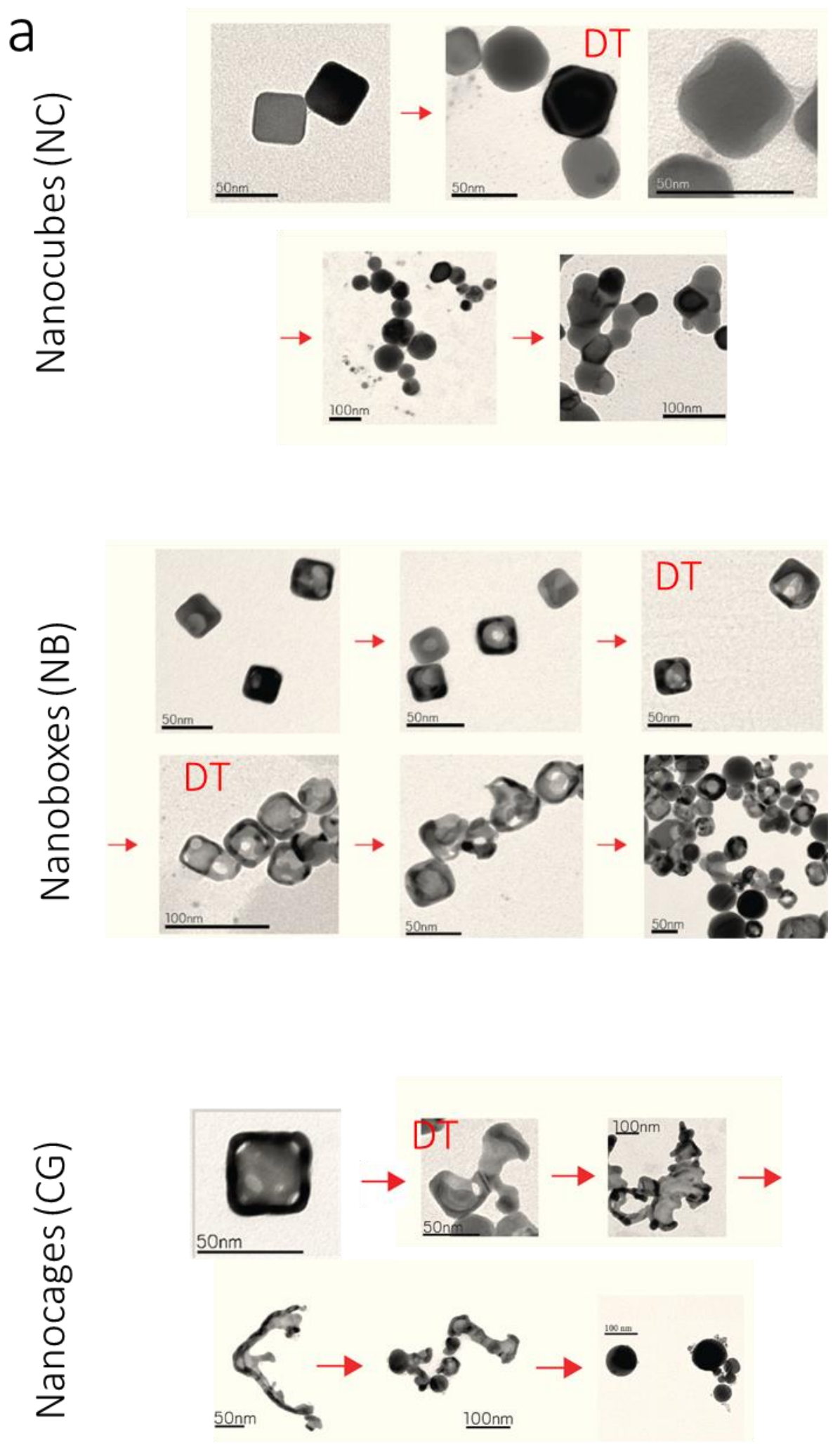

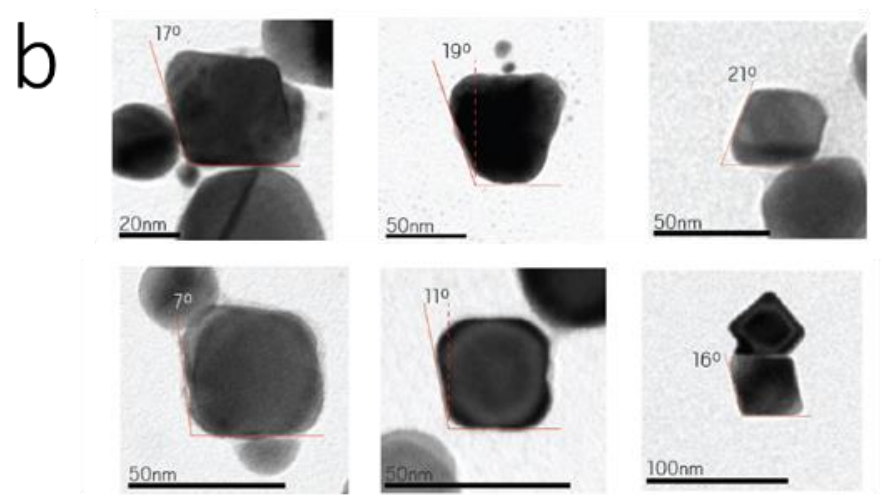

C
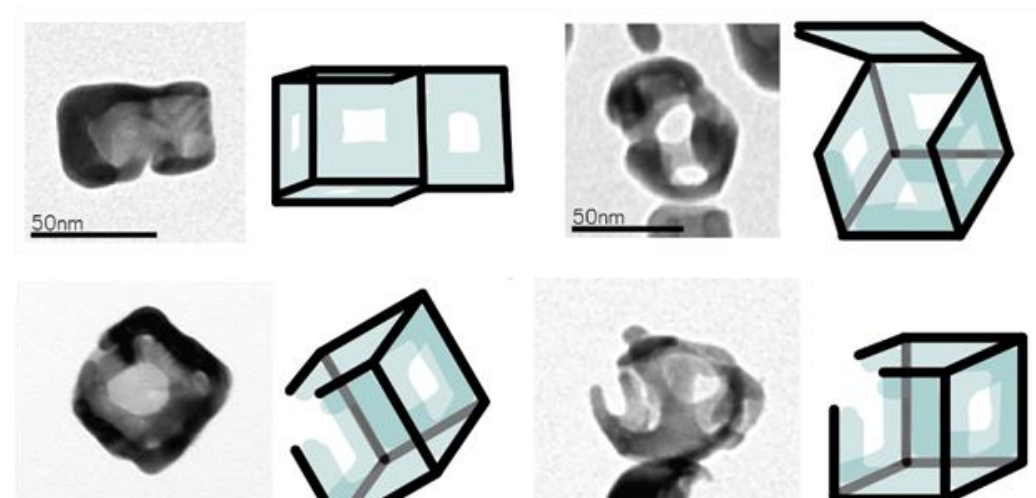

$50 \mathrm{~nm}$
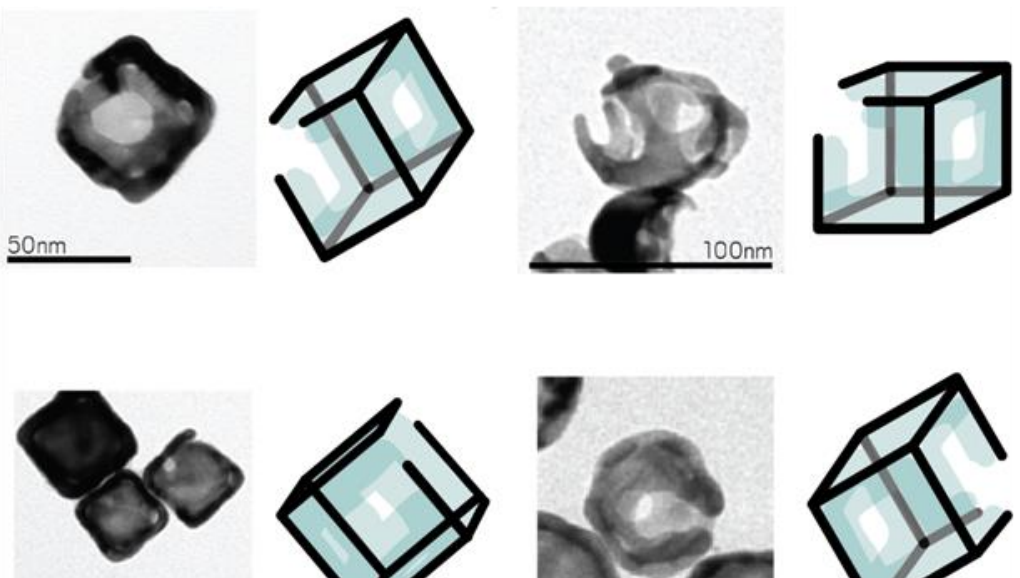

$\underline{20 n m}$
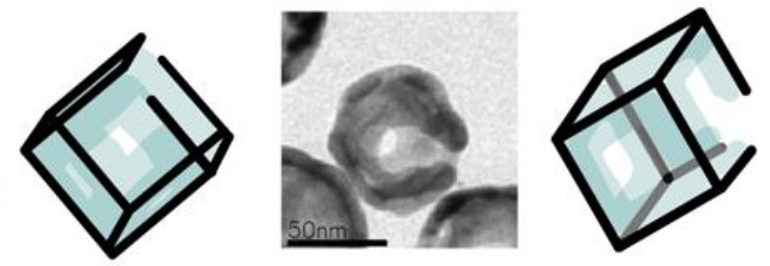

d

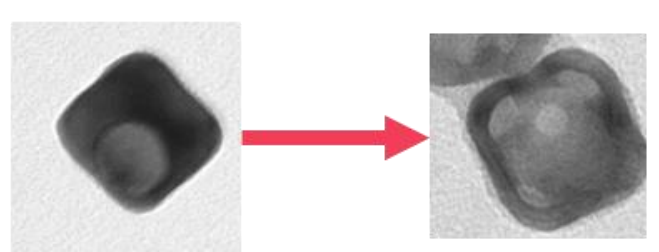

Figure 82. (a) Particle modification stages during the course of exposure in nanocubes (NC), nanoboxes (NB) and nanocages (CG). Results show deformation threshold (DT) (in between spectral threshold (ST) and melting threshold (MT)) specifically modifies particles at their plasmonic hot-spot locations at corners or cavities. (b) associated Lattice displacements in NCs at DT (c) Corner and edge fragmentations in CGs at DT (d) localized cavity enlargement in NBs to form ultrashort pulse assisted CGs at DT. 
All these modifications were associated with their corresponding spectral changes (Figure 72). At DT, the LSPR peak of NCs at $357 \mathrm{~nm}$ vanished along with blunting of corners. This evidence confirms $357 \mathrm{~nm}$ peak as a signature of sharp corners in cubes. Similarly, CGs at DT showed a red shift towards $500 \mathrm{~nm}$ corresponding to fragmentation of corners. In NBs the cavity expanded uniformly within the particle to form laser induced CGs. This is the first experimental evidence for laser induced nanobox to nanocage transformation in literature. Overall, these results prove that DT is indeed the energy level, where the LSPR modes absorb photonic energy at hot-spots causing localized deformation. Additionally, because localized deformation is not observed at MT, it can be considered as an energy level, where particles receive enough heat energy to undergo rapid melting. This differentiates deformation and melting stages and confirms the existence of discrete interactive-level of energy in these nanoparticles. Overall, these data evidently show specificity of laser wavelength to the LSPR wavelength of the cubic nanoparticle.

In order to further investigate modification of cavity in NBs, we increased the $E_{\text {pulse }}$ in a stepwise manner. For each $5 \mu \mathrm{J}$ step, particles were analyzed for spectral changes and modifications were characterized. We measured the change in cavity diameter using image analysis and plotted it as a function of incident energy. In order to normalize for all particles observed, the cavity size was measured relative to the edge length of each particle. The resulting ratio between cavity volume and particle volume was measured and represented as the effective void fraction $(\Phi)$. Results evidently show a minimum activation energy of $50 \mu \mathrm{J}$, was required to increase the cavity size (Figure 83). Therefore, the changes occurring inside NBs is in fact, dependent on coupled-SPR 

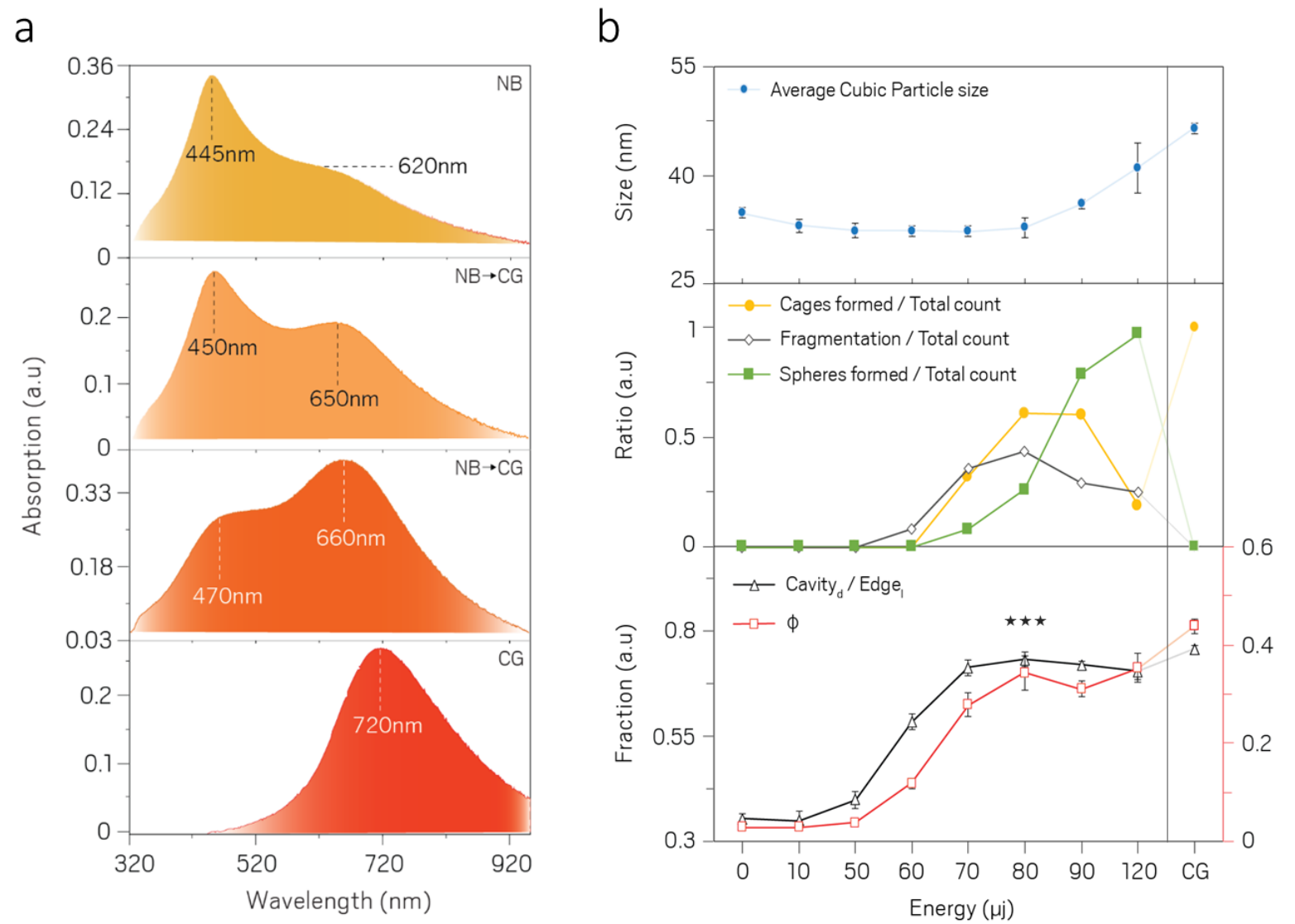

Figure 83. (a) Gradual shift in UV-vis absorption profile for CG formation using NB using wet chemical synthesis (b) Formation of CGs from NBs using laser pulses. (Top-bottom) Graphs represent average size as a measure of edge length, transformation statistics and cavity profile at various incident energies. The last column in all graph represent CG statics from wet synthesis for comparison. ${ }^{\star \star} \mathrm{p} \leq 0.001$ and the values were analyzed using one-way anova

enhanced absorption of laser light. At around $80 \mu \mathrm{J}$ most of the NBs were transformed to CGs. Although the fragmentation at this energy level was highest the average particle size remained same. Higher energies however, expanded the size of particle due to heating and formed spheres, as observed at MT (Figure 83). It is interesting to note that, the average particle size of CGs synthesized using wet chemical process was larger than laser synthesis (Figure 83). This size increase is attributed to gold deposition over the surface during wet synthesis. Whereas, laser synthesis efficiently etched the nanoboxes at the nanocavity surface without resulting in a size change. Remarkably, these results 
fit our simulated models that showed high absorption around the cavity (Figure 75). These results also corroborate similar simulated data using DDA approximations in a previous study ${ }^{165}$. That study postulated light amplification (SPASER) within nanobox cores. Together, the data demonstrate that, small nanocavities linked to nearby LSPR modes can trap light. This process allows precise maskless photolithography to take place in the presence of ultrashort light pulses. This experiment also confirms the role of nanocavities as a conduit for LSPR coupling in cubic particles. The LSPR coupling eventually initiates a resonance cascade, causing light confinement at sub-wavelength space.

\subsubsection{Characterization of single nanoparticle deformations}

Similar to a previous study we monitored modification in solution using size distribution analysis $^{166}$. Results show a sharp increase in fragmented particles especially in CGs (Figure 84). CG-fragmentation led to formation of nanoframe like beam structures previously seen during our synthesis of NFs (Figure 58). In fact, size distribution of CGs at DT resembled with the distribution of NFs synthesized using wet chemical process (Figure 84). AFM topography analysis of these fragments also showed very similar beam like fragment seen in NF synthesis (Figure 85). Additionally, we characterized particles based on their UV visible absorbance. These results were similar to the transmittance spectra recorded during exposure. However, both these techniques provide an averaged

result, from a mean population distribution. To understand the actual changes in the nanoparticles with more specificity and sensitivity we would need to characterize SPR shift of individual nanoparticles. 
a

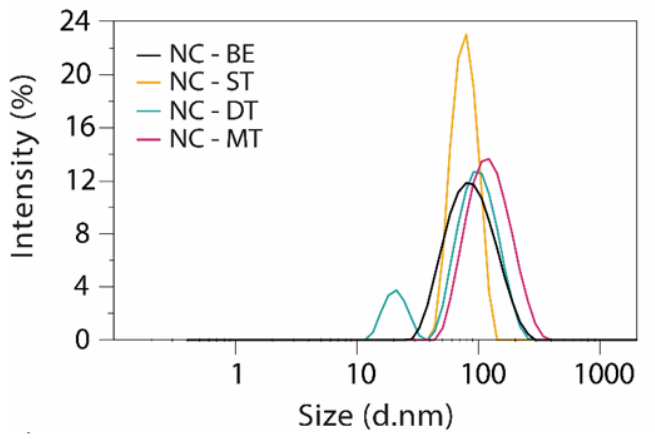

C

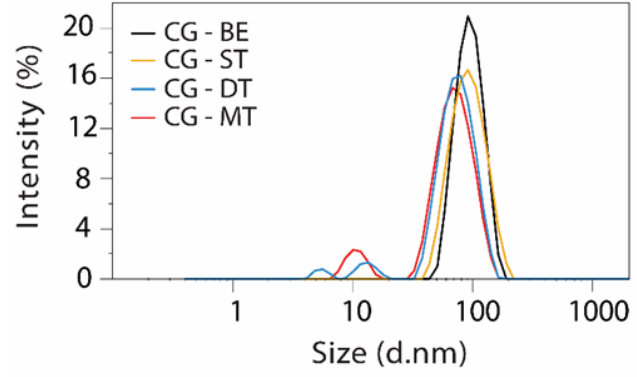

b
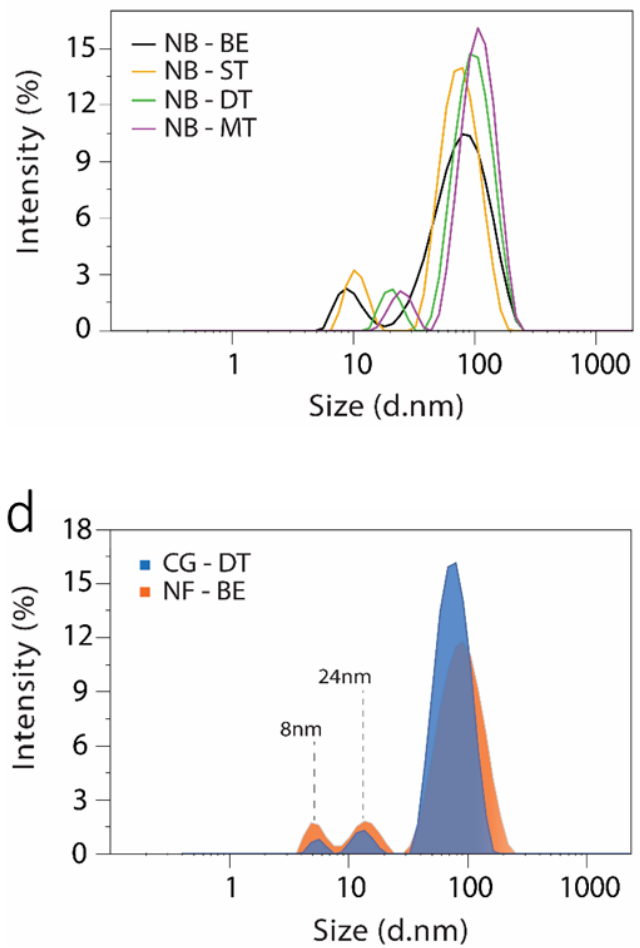

Figure 84. Particle size distribution profiles for (a) nanocubes, (b) nanoboxes, (c) nanocages and (d) comparison with as synthesized nanoframes. BE, ST, DT and MT stand for Before exposure, Spectral threshold, Deformation threshold and Melting threshold respectively.

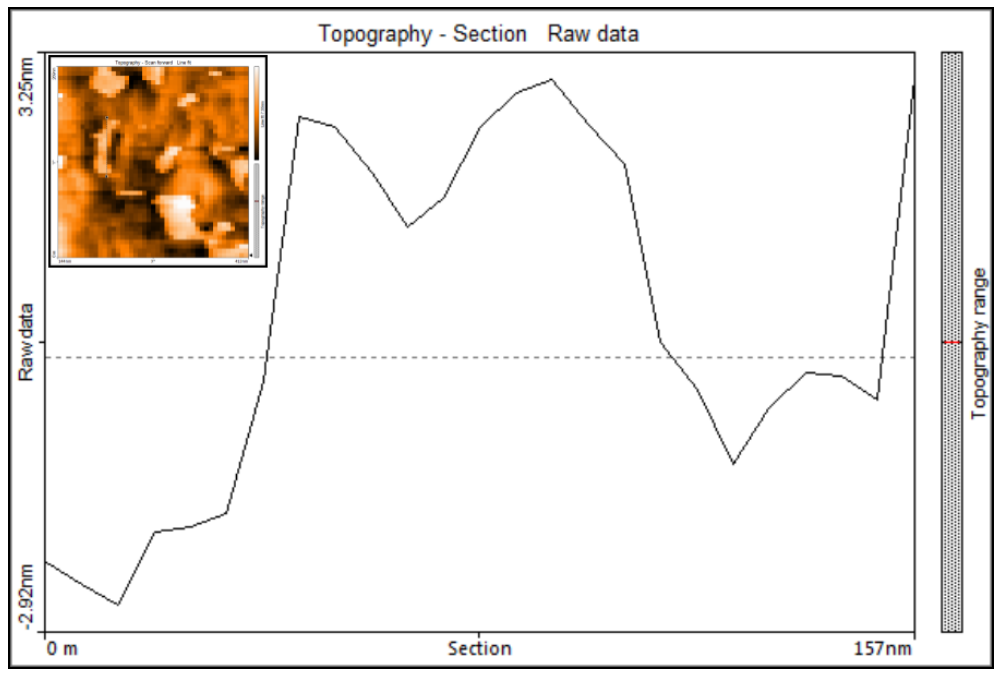

Figure 85. AFM surface topography profile for a nanobeam formed during nanocage fragmentation revels asymmetrical bending and flattening of structures. 
Therefore, using Rayleigh scattering spectroscopy we studied particle modifications in single nanoparticles ${ }^{123,167}$. This technique relies on the ability of single nanoparticles to scatter light ${ }^{135}$. As discussed before, particles size below the electron's mean free path absorb more photons than scattering. However, cubic particles that have a high volume as well as aspect ratios, have shown to scatter light intensely owing to the electromagnetic field enhancements from their edge lengths (Figure 86). Using hyperspectral analysis, the Rayleigh scattered light of nanocubic particles were measured (Figure 87). We observed spectra corresponding to LSPR peaks and detected changes in the SPR peak shifts at ST, DT and MT (Figure 87). As expected the changes in DT and MT were more pronounced. Results evidently show formation of spheres at MT (Figure 87). These results were similar to a previous study that documented formation of silver and gold-silver alloy spheres ${ }^{168,}$ 169. Additionally, SPR shifts at DT were in agreement with UV-visible shifts. Similarly, results from single nanoparticle spectra at BE were in direct agreement with simulated spectra using finite domain time domain (FDTD) analysis (Figure 88). This proves accepted fit between simulated models and experiments.

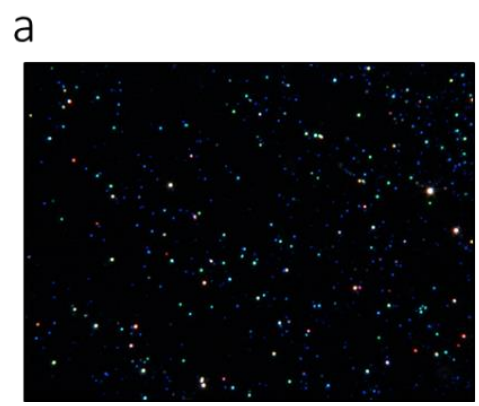

b

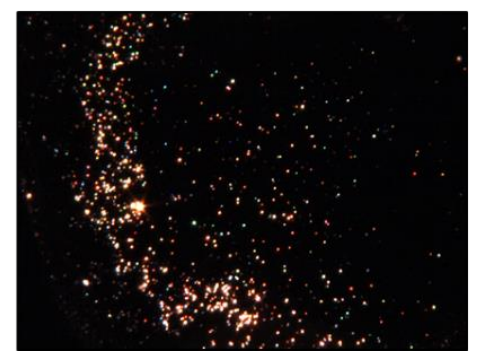

C

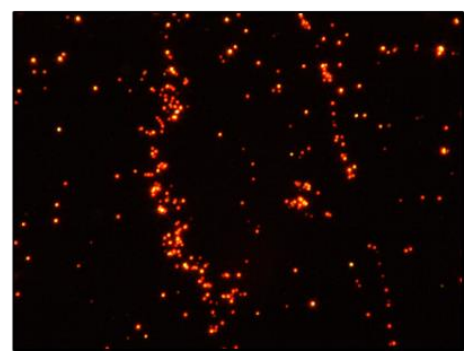

Figure 86. Rayleigh scattering of light by (a) NCs, (b) NBs, and (c) CGs (dark-field, 100x oil) 
a
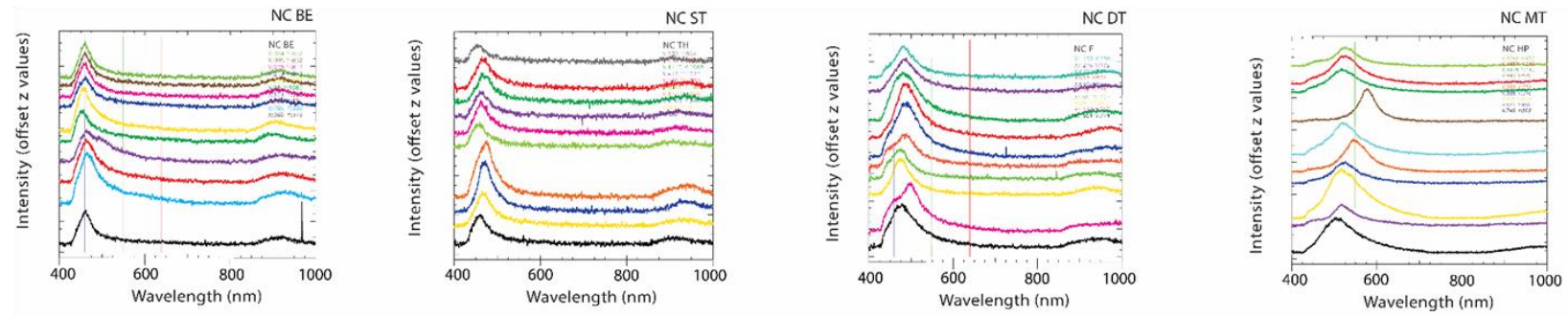

b
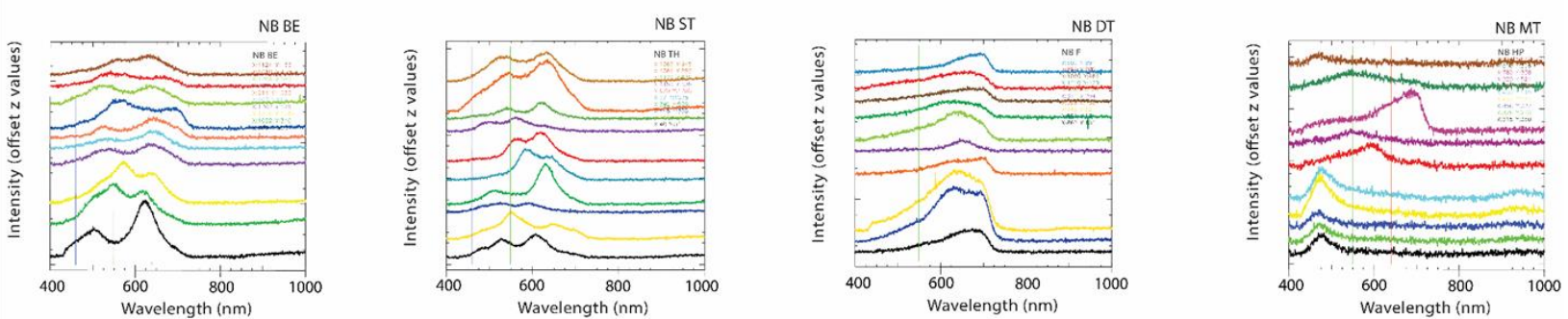

C
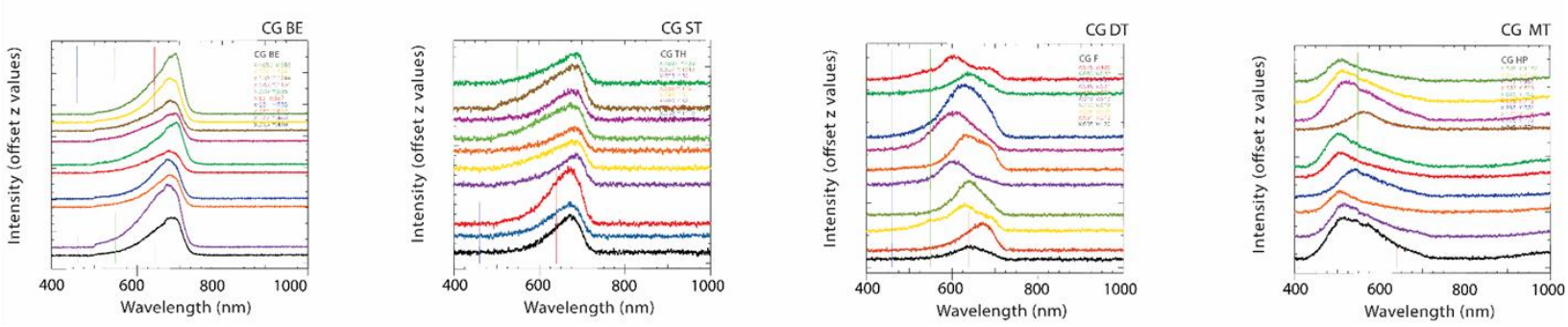

Figure 87. Rayleigh scattering of single nanoparticles (a) NCs, (b) NBs, and (c) CGs. BE, ST, DT and MT stand for Before exposure, Spectral threshold, Deformation threshold and Melting threshold respectively.

a

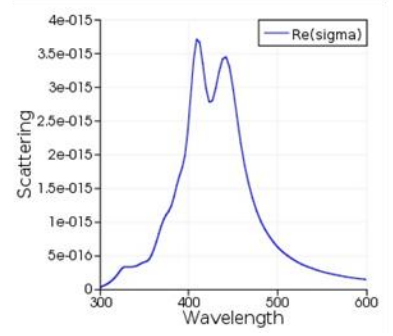

b

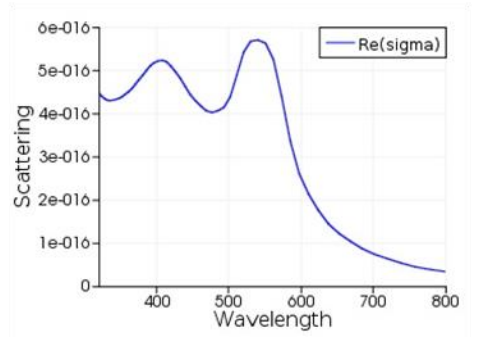

C

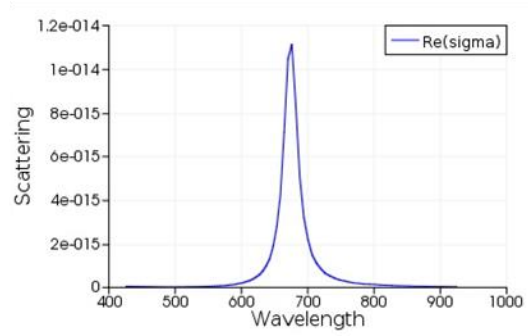

Figure 88. Scattering simulation for (a) NCs, (b) NBs, and (c) CGs. Results show a similar peak for NCs at $450 \mathrm{~nm}$, double peak for NBs at $420 \mathrm{~nm}$ and $580 \mathrm{~nm}$, and a $700 \mathrm{~nm}$ peak for CGs. 

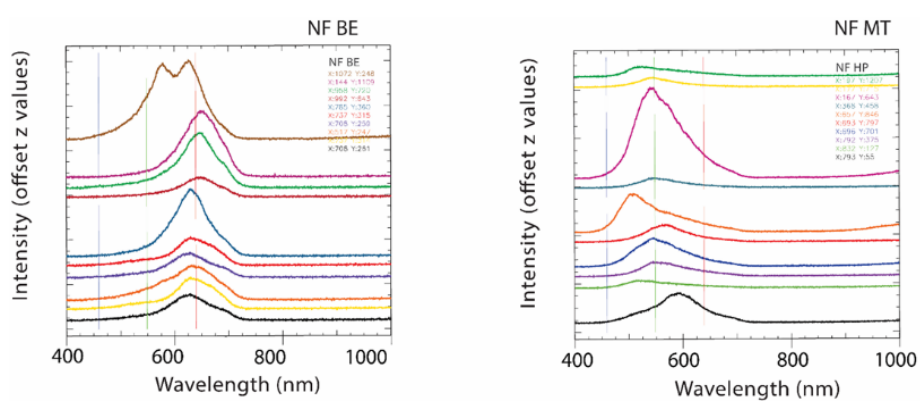

Figure 89. Rayleigh scattering of single nanoframes. BE and MT stand for Before exposure and Melting threshold respectively.

In the single nanoparticle spectroscopy data, CGs at DT were similar to NF before exposure (Figure 89). The result obtained signify the formation of frames by fragmenting nanocages using laser pulses. Interestingly, the SPR peak for NBs at DT were analogous to CGs before laser treatment (BE) (Figure 87). As shown in Figure 90, computed 3D near-field enhancements profiles from our simulations clearly demonstrate that NBs undergo selective lasing at the cavities. This was reflected in an earlier study regarding the coupling of interior and exterior plasmon modes ${ }^{170}$. Together, with single nanoparticle spectroscopy data and these simulations, results evidently corroborate with experimental TEM observations for the transformation of NBs to CGs after exposure at DT.

a

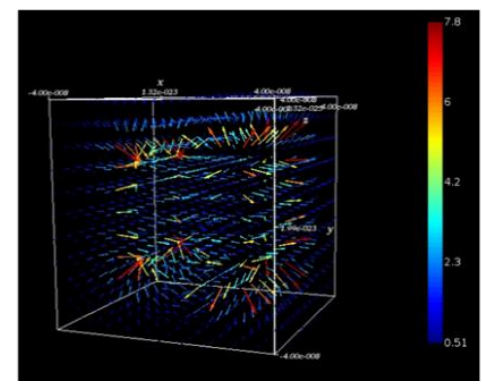

b

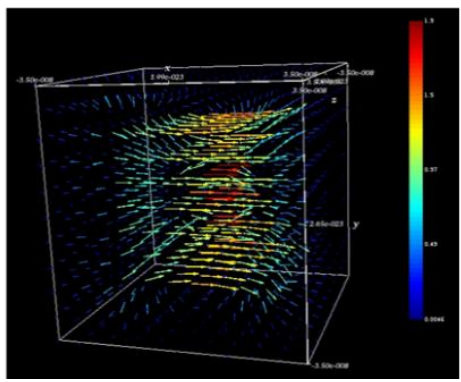

C

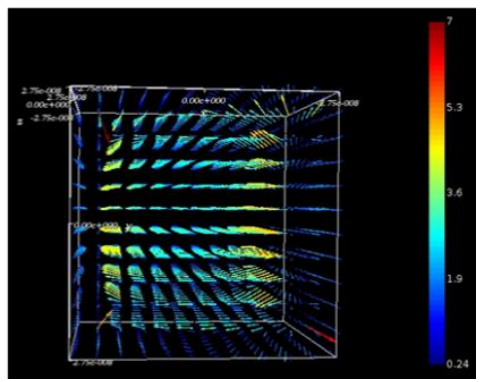

Figure 90. Computed 3D Near-field enhancements for (a) NC, (b) NB and (c) CG. Results clearly show selective lasing in cavities of NB as compared to NC and CG. 
Overall these results confirm the modifications at the single nanoparticle level. To further investigate the modifications at the atomic scale in single nanoparticles we analyzed exposed particles using high resolution TEM techniques.

\subsubsection{Lattice rearrangement in single nanoparticles}

In order to image at the atomic scale, the nanoparticles were imaged using STEMHAADF. TEM images for NC before exposure revealed a crystalline structure indicating formation of a uniform crystal from a seed (Figure 67). At DT, laser treated NCs were modified at the corners, but this did not affect the crystalline structure within the particle (Figure 91). The result obtained confirm localized modifications at the atomic scale. Furthermore, NCs transformed into spheres at higher energies (Figure 92). As expected, spheres that formed after melting showed high crystallinity (Figure 92). Unlike NCs, images for NB and CGs before exposure revealed a polycrystalline structure owing to gold

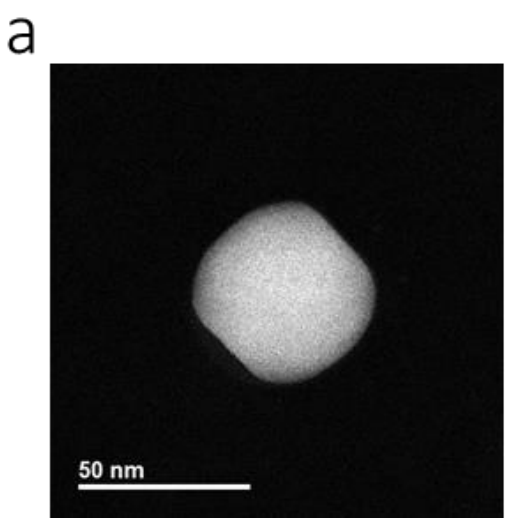

b

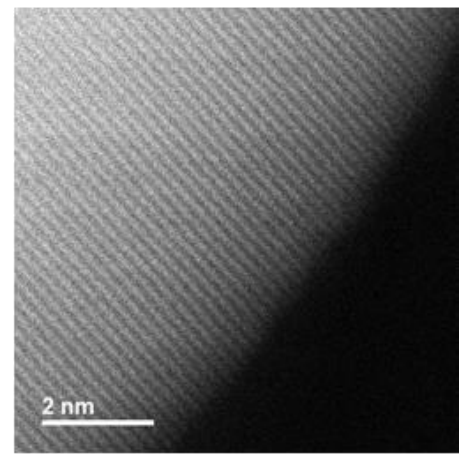

Figure 91. STEM-HAADF images for (a) deformed NC and (b) expanded view of the particle showing uniform crystalline lattice structure. 


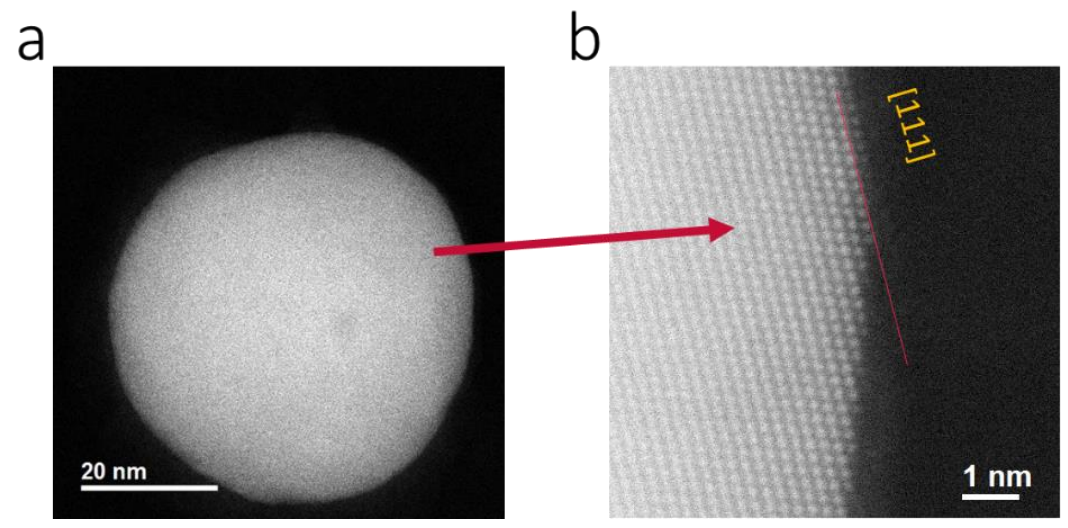

Figure 92. STEM-HAADF images for (a) melted spherical NC and (b) expanded view of the particle showing uniformly rearranged crystalline lattice structure.

deposition and formation of a gold-silver alloyed interior (Figure 68). At DT, both NB and CG after laser treatment showed LSPR based localized deformations and fragmentation at corners and cavities (Figure 93). Along with deformations at curvatures, defect boundaries at bending zones were observed (Figure 93). Previous studies have shown that defect boundaries can act as a seed for fragmentation ${ }^{147}$.
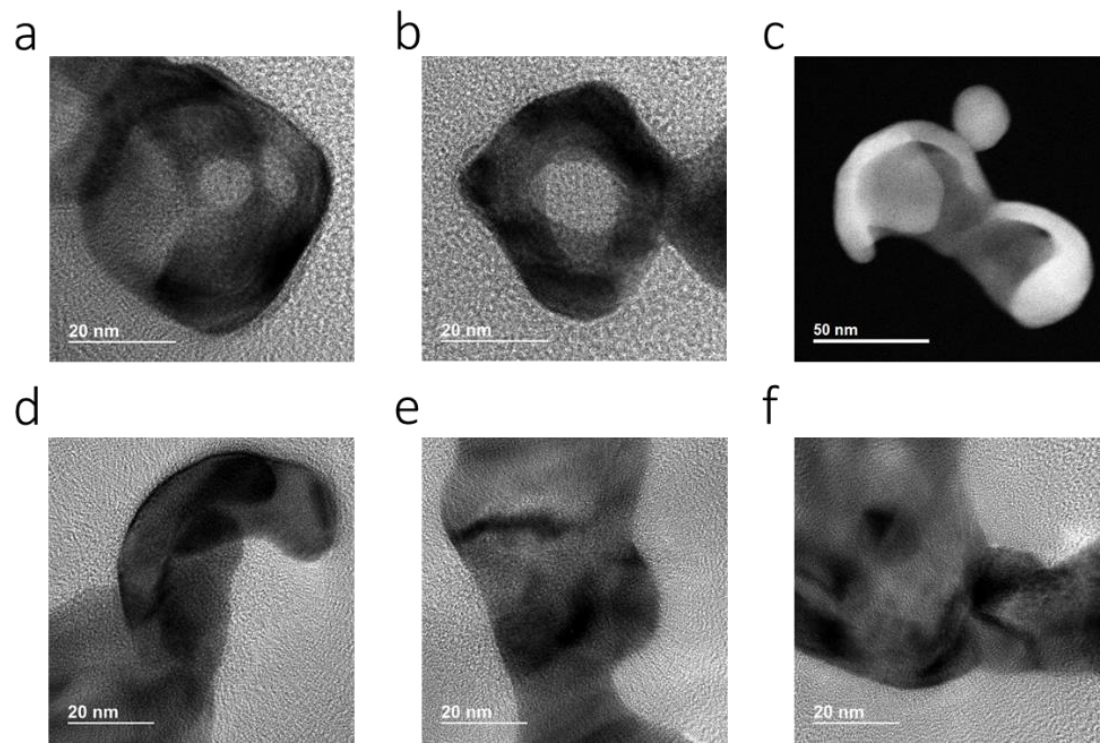

e
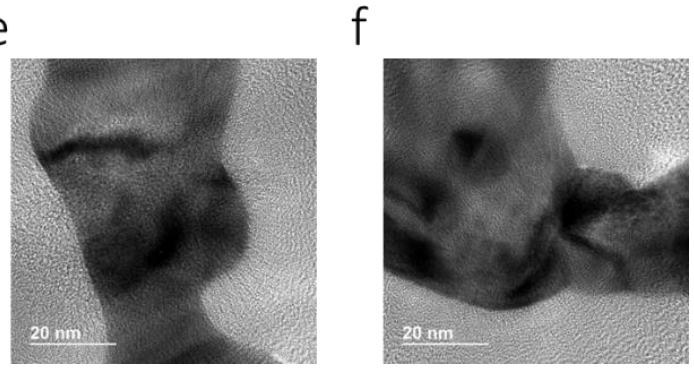

Figure 93. STEM-HAADF images for (a) modified NB (b) modified NB (c) an opened CG and its associated (d-f) bended joints. 

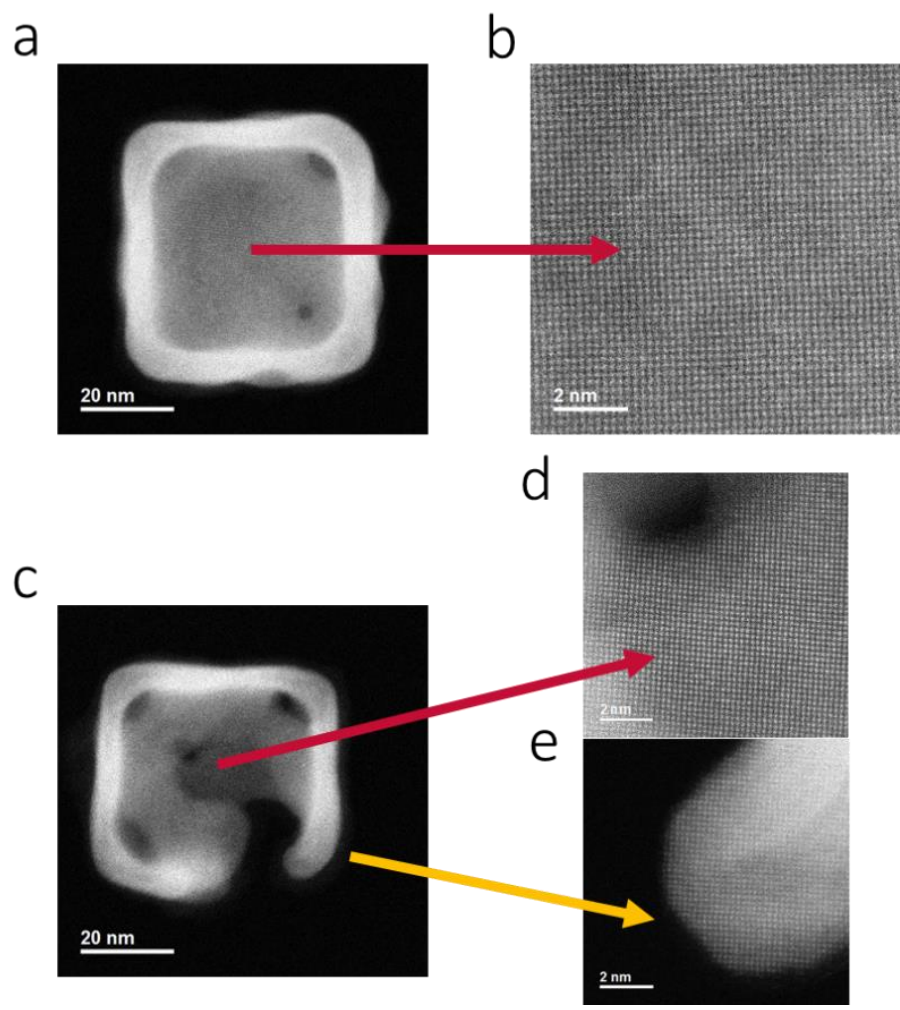

Figure 94. STEM-HAADF images for (a) unexposed nanocage (CG) with the (b) expanded view showing a uniform polycrystalline lattice (c) a deformed CG with its expanded views showing (d) uniform atomic columns of mixed Au and $\mathrm{Ag}$ atoms similar to unexposed CG and (e) similar structure at the fragmented corner indicating a columbic explosion or electrostatic repulsion at play.

Interestingly, the nanoparticles still maintained a polycrystalline network and did not show any signs of melting (Figure 94). Uniform lattice columns of gold and silver atoms were found in the facets of both particles (Figure 94). Because the localized deformations did not affect the whole lattice, these results confirm that, modifications at DT are highly localized. Additionally, the fragmented joints at corners were also observed to be intact (Figure 94). This observation indicates the fragmentation mechanism could be a athermal columbic explosion or electrostatic repulsion causing ultrafast atomic expulsion at the specific location. As a next step, the elemental composition of gold-alloyed particles namely, NBs and CGs were analyzed using STEM-EDS and EDX. 


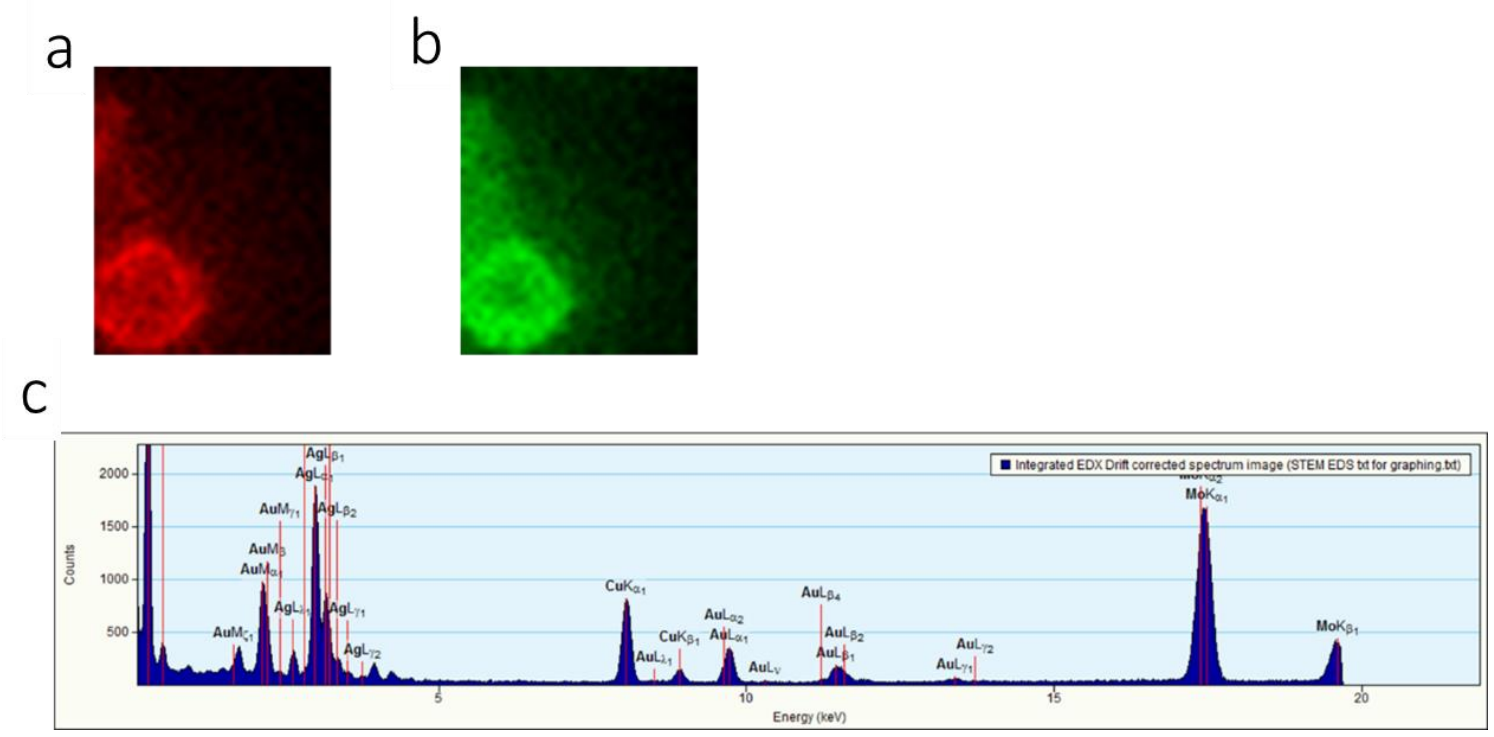

Figure 95. STEM-EDX analysis for a modified NB: (a) Thick gold exterior (b) a dispersed Ag interior (c) an alloyed composition for the particle.

(Figure 71). Results confirmed that the composition of gold and silver is preserved throughout the NB structure after exposure at DT (Figure 95). Interestingly, in NBs samples sub-10 nm silver nanoparticles were found (Figure 96). This results indicates silver leaching in nanobox cavities during the transformation step. Furthermore, TEM images showed presence of amorphous carbon jets protruding from curvature points especially corners (Figure 97). The amorphized carbon, previously shown to be hydrophobic, explains
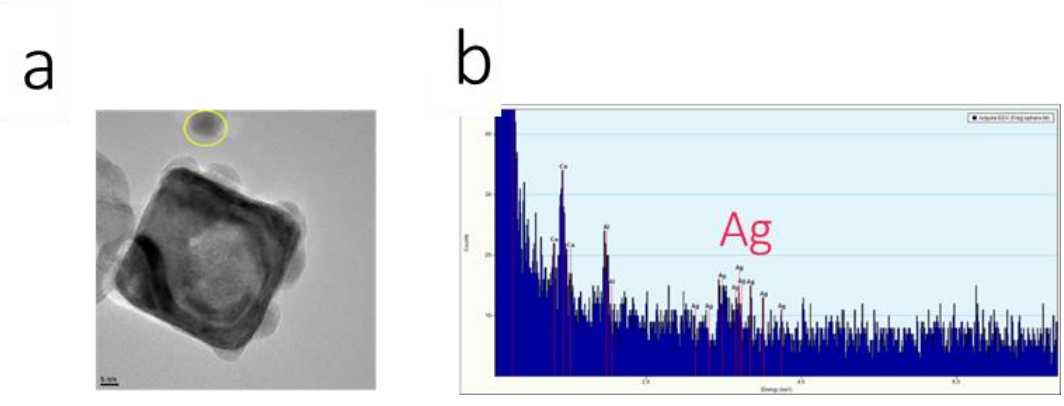

Figure 96. STEM-EDX analysis for a (a) spherical particle indicates (b) silver composition devoid of gold. 


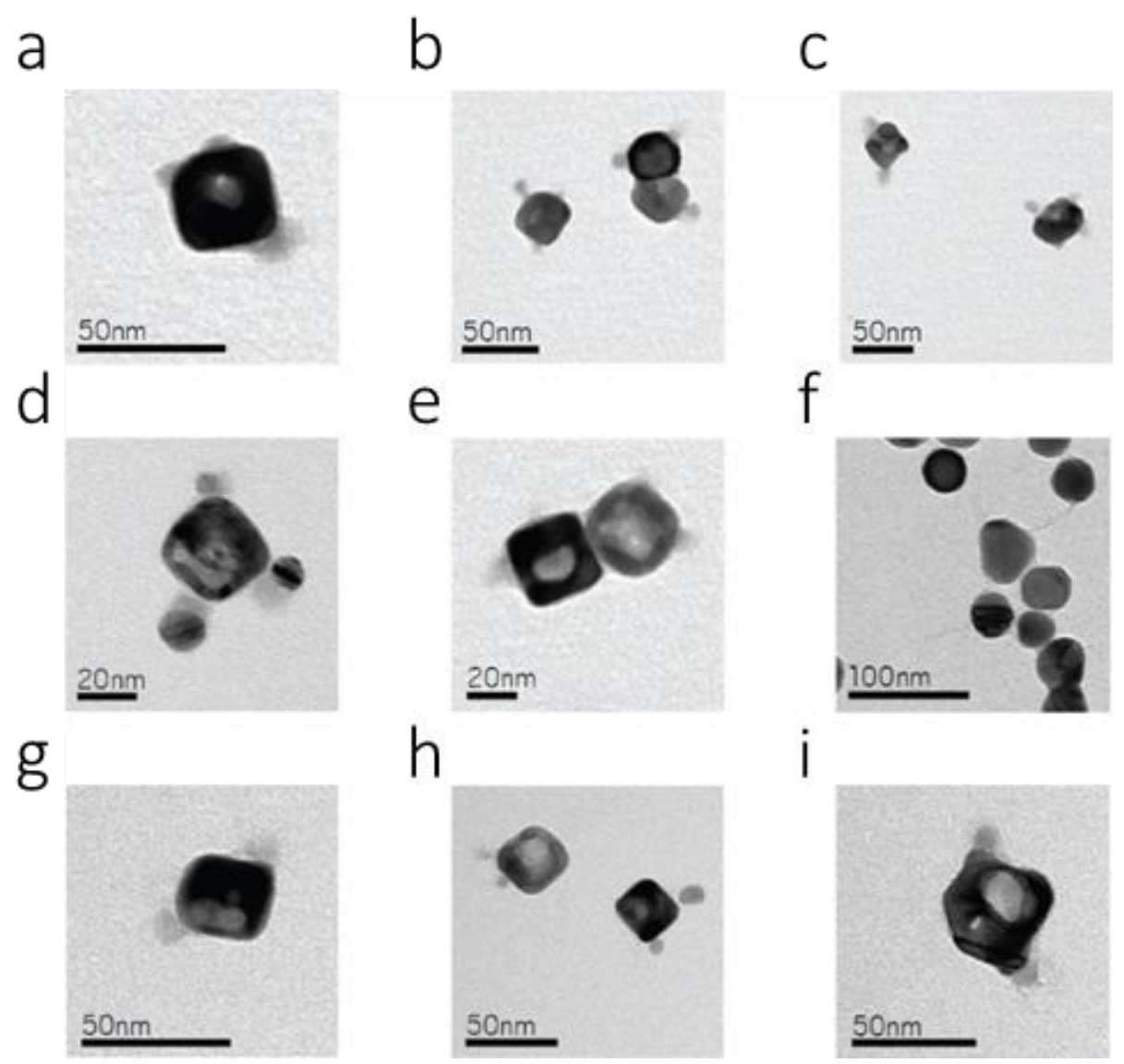

Figure 97. TEM images (a-i) show presence of amorphized PVP jets at the surface of modified particles exposed at DT.

presence of particle aggregates in solution after exposure at MT (Figure 98) ${ }^{171}$. These amorphous carbon formation was also confirmed in native nanoparticles during electron beam exposure in HR-TEM. Coincidently, these jets were only noticed in particles after exposure to DT. It is likely these jets were formed by amorphization of PVP due to hotspot energy release. Such amorphization has been previously documented in another laser study with PVP coated silver nanoparticles ${ }^{171}$. Together, they signify PVP heating at the curvatures that may be responsible for spectral shifts seen at ST. These results strongly demonstrate localized absorption of photonic energy at the corners and thin faces. 

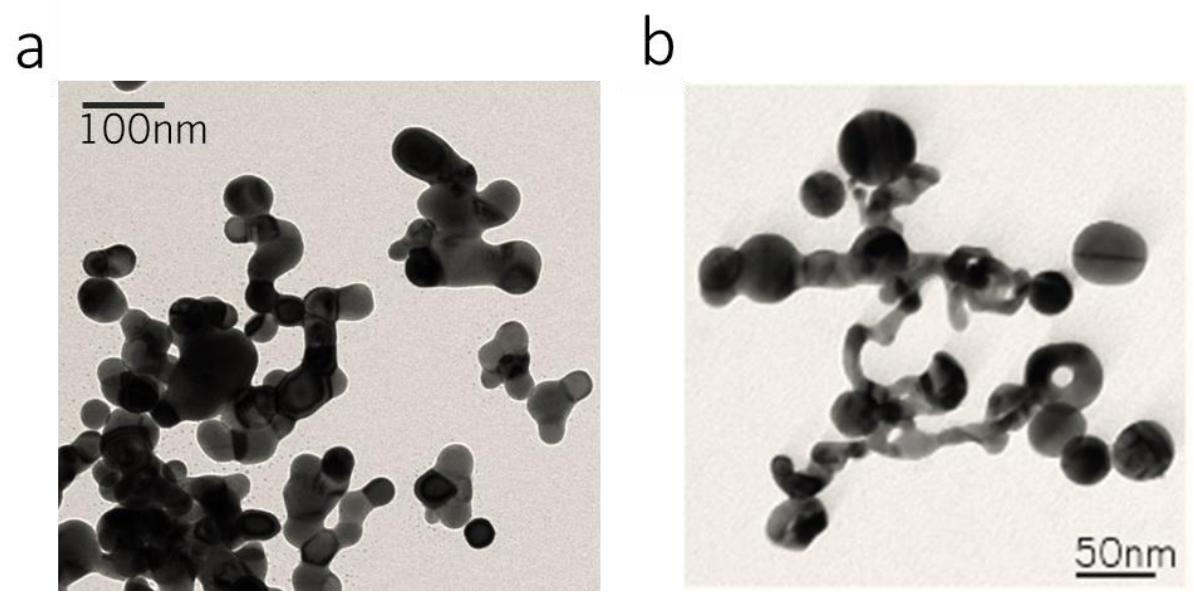

Figure 98. TEM images showing melted aggregated networks formed at MT (a) melted NC (b) melted CG.

\subsubsection{Mechanism of excitation in cubic nanoarchitectures}

Results from spectroscopy reveal discrete activation thresholds. Characterization of modified particles also confirm localized modifications due to LSPR association at DT. HR-TEM results also corroborated these localized modifications and show no rearrangements in the crystal lattice at DT. These results strongly suggest a non-thermal nature of excitation. In other words, it would mean the melting process at MT is thermal in nature. Therefore, any heat lost from the lattice during the transfer to the surrounding medium would have to be compensated with an increase in activation thresholds. Based on this theory, we hypothesize that activation thresholds for DT would not be dependent on the thermal conductivity of the surrounding medium. In order to prove this hypothesis, we exposed the nanoparticle to ultrashort pulses in different solvents. By changing the solvents with different thermal conductivity, we radically change the heat loss characteristic of the system. The laser treatment was performed in water $\left(0.6 \mathrm{Wm}^{-1} \mathrm{~K}^{-1}\right)$, methanol $\left(0.2 \mathrm{Wm}^{-1} \mathrm{~K}^{-1}\right)$ and butanol $\left(0.15 \mathrm{Wm}^{-1} \mathrm{~K}^{-1}\right)$. Because the thermal conductivity 

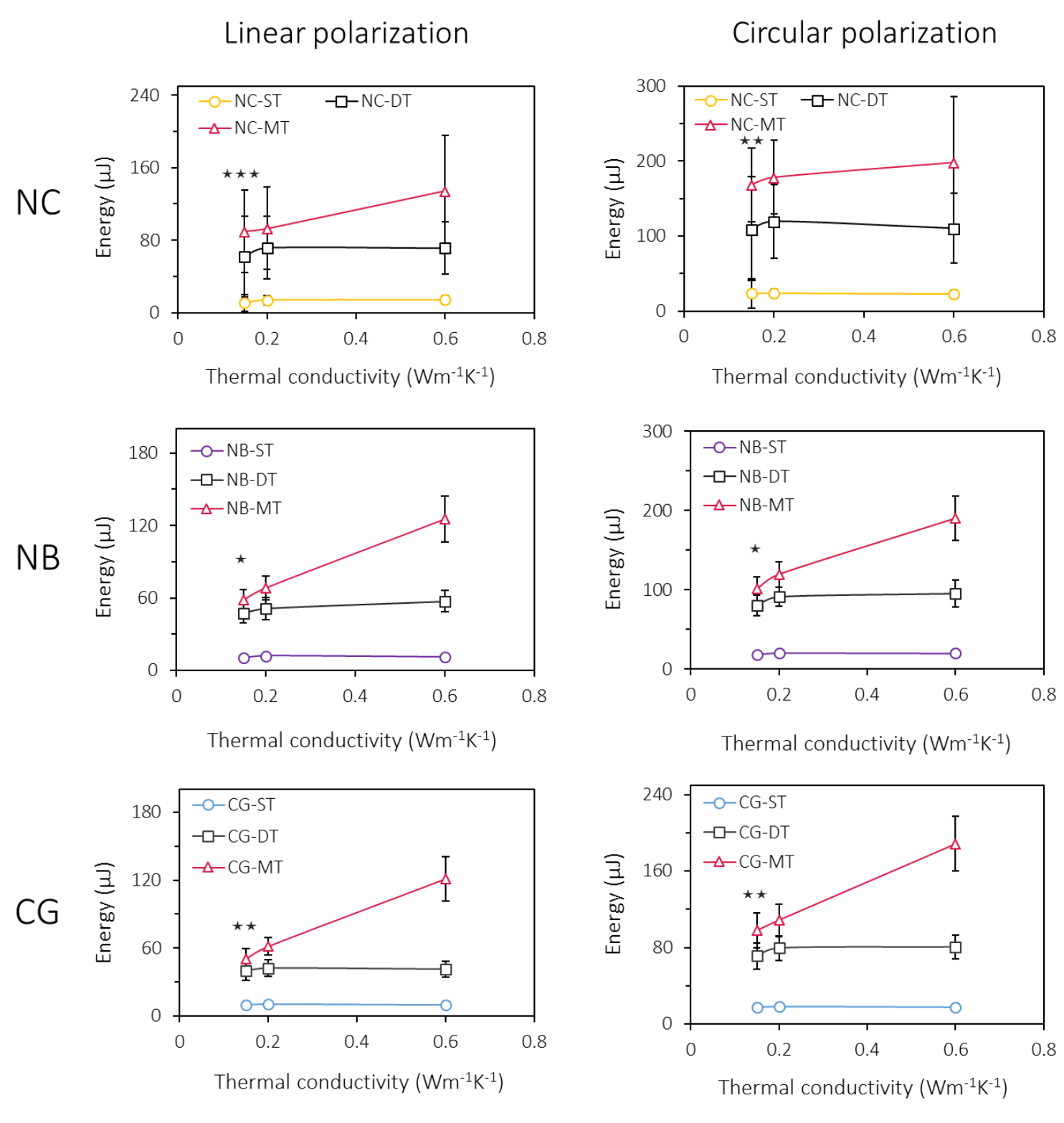

Thermal conductivity $\left(\mathrm{Wm}^{-1} \mathrm{~K}^{-1}\right)$

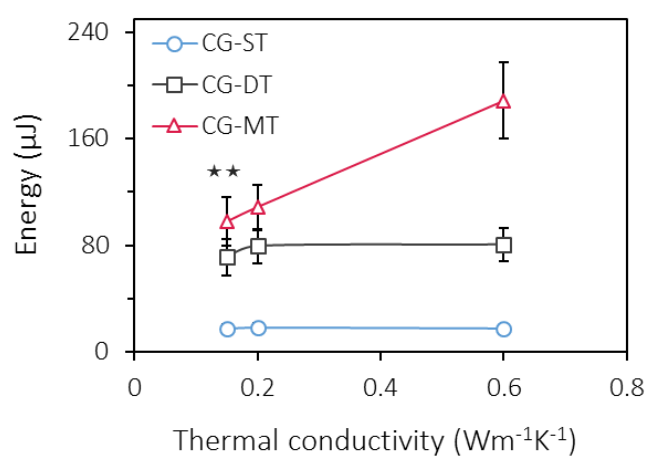

Figure 99. Activation thresholds for $\mathrm{NC}, \mathrm{NB}, \mathrm{CG}$ using linear $(\mathrm{Li})$ and circular (Ci) polarization exposure by femtosecond light pulses in three different mediums of varied thermal conductivities. Results indicate an increase in required activation energy with increasing thermal conductivities. ${ }^{\star}$ indicate $\mathrm{p} \leq 0.05,{ }^{\star \star}$ indicate $\mathrm{p} \leq 0.01$, and ${ }^{\star \star}$ indicate $\mathrm{p} \leq 0.001$ and the values were analyzed using one-way anova

between water and the other two solvents is 2 fold we expect an adjustment in activation thresholds, required for thermal changes to occur in the lattice. Although the difference between methanol and butanol is 1.3 fold, we expect a small change that could show how 
sensitive the test is. Overall, higher thermal conductivity would lead to higher heat loss to surroundings, and therefore increase the energy threshold to compensate for melting. Indeed, our experiments evidently show an increase in activation thresholds for all three particles in water for MT alone (Figure 99). The percent difference for NC, NB and CG at MT between water and methanol were $36 \%, 59 \%$, and $65 \%$ respectively. The percent difference for methanol to butanol for NC, NB and CG at MT were 3\%, $15 \%$ and $20 \%$ respectively. As such, activation thresholds at ST and DT in water, were higher comparable to both methanol and butanol. As changes in ST and DT were negligible, these results signify that modification at DT are indeed non-thermal in nature. This means that as ultrashort pulses are absorbed at localized hot-spots on the cubic particles, the intrinsic crystal field is affected at the curvatures. The resulting local-phonon excitation will ultimately decrease the binding energy $(\mathrm{Au} ; 3.375 \mathrm{eV} /$ atom $)$ at the hot-spots while, the lattice relatively remains cold ${ }^{147}$. This suggests that, resulting effects will not generate a photothermal effect. Therefore, the localized deformations may be due to coulomb explosion or electrostatic explosion that are associated with non-thermal and nonequilibrium conditions. Taken together, the data indicates that biomolecules such as proteins near the surface will not decompose due to particle thermal confinement. As such particle deformations might take place before the time of thermal denaturation $\left(\tau_{D}\right)$ of most proteins that is given by (Equation 9) ${ }^{172}$ :

$$
\tau_{D}=\frac{\hbar}{\kappa_{B} T} \exp \left(\frac{\Delta H-T \Delta S}{R_{g} T}\right)
$$

where $\tau_{D}$ is inversely proportional to temperature T; $\Delta H, \Delta S$ are the difference of enthalpy and entropy of the protein state, $\hbar$ is the planks constant, and $R_{g}$ is the universal gas constant. These factors predict a protein denaturation time of $1 \mathrm{ps}-1 \mu \mathrm{s}^{172}$. Considering 


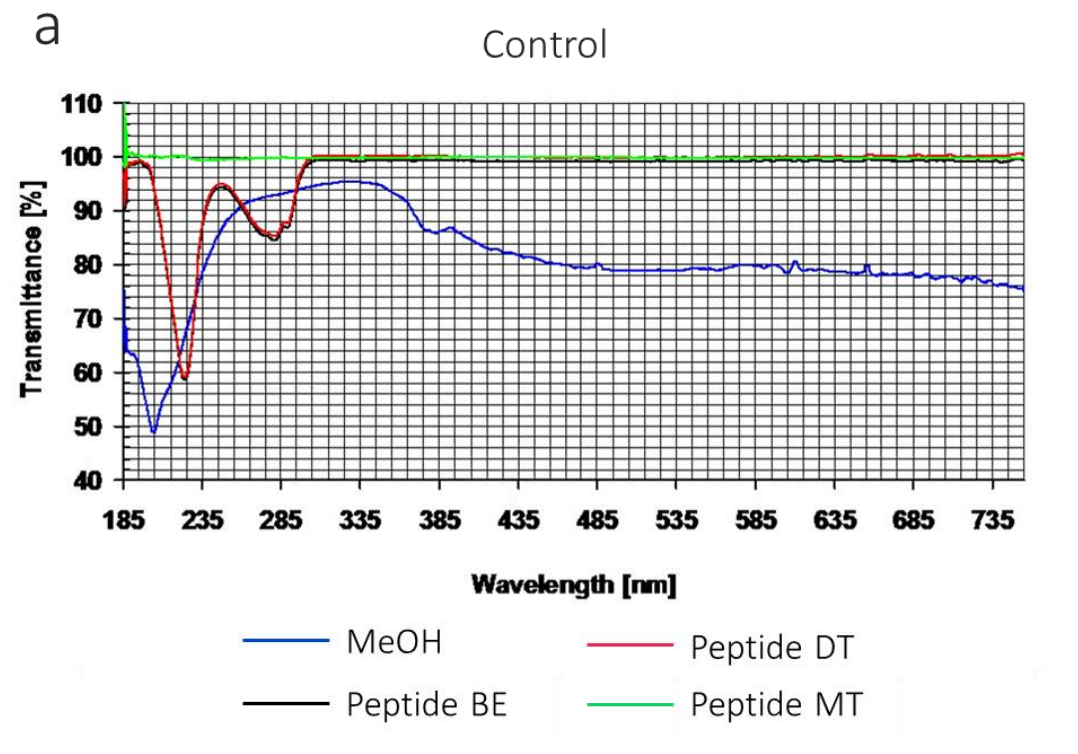

b

\section{Release}

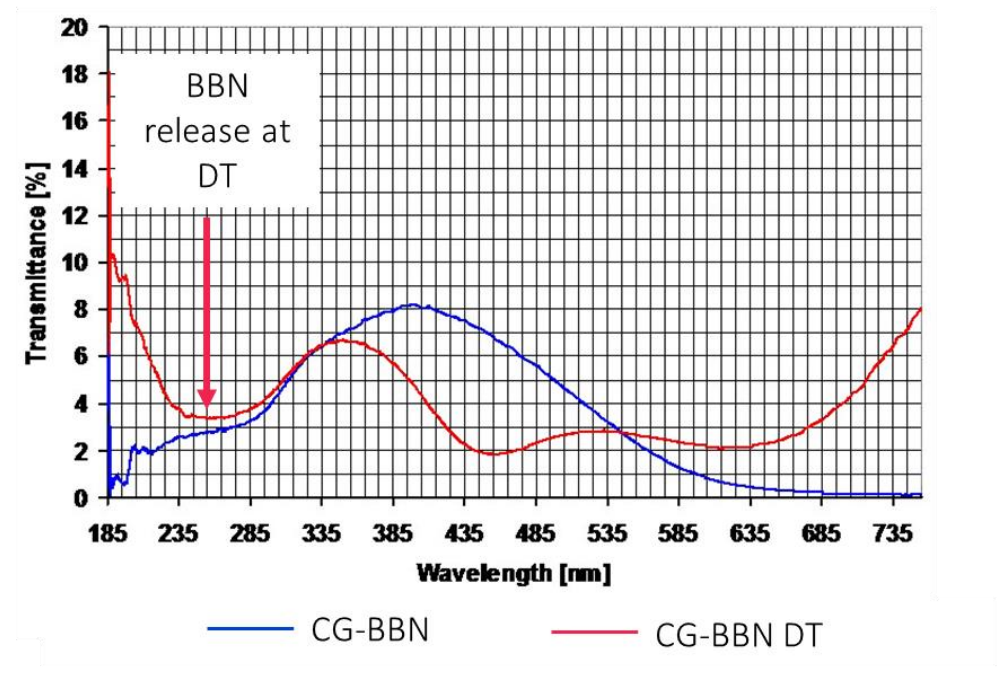

Figure 100. Transmittance spectroscopy of (a) Bombesin peptide as control showing a peak at $220 \mathrm{~nm}$ and (b) CGbombesin that was exposed at DT showing a peak at $245 \mathrm{~nm}$ suggesting release of peptide.

deformations take place at femtoseconds, DT would be ideal for controlled release of molecules. Based on these results we treated peptide (short protein sequence) conjugated gold nanocages with ultrashort pulses at DT to observe peptide-release. CGs were surface functionalized with Bombesin peptide using thiol linkers that have affinity for gold atoms. 
CG-BBN were then irradiated at DT and monitored. Results from transmittance spectra show release of peptide from surface indicating very low laser energy $(<50 \mu \mathrm{J})$ is required for controlled release (Figure 100). These results prove that cubic nanoparticles can be used for controlled delivery of drugs at very low laser fluence without causing additional photothermal effects.

\subsection{CONCLUSION}

In this study we synthesized three different functional cubic nanoarchitectures. We characterized the LSPR modes and electric field enhancements for these particles using nanoscale and atomic scale characterization techniques. We found three discrete energy levels in these particles using spectroscopy, that indicate presence of LSPR coupling and localized light confinement. Furthermore, we confirm confined absorptions at extremecurvatures, are responsible for localized modifications. Additionally, we also explain the mechanism of excitation as non-thermal in nature. Data suggests that nanobox geometries can guide or focus light. Overall, we show that using shape of nanostructures, one can design LSPR hot-spots for optimal absorption of ultra-low power laser radiation. By using localized absorption at very low intensities of energy we have developed a method for nanoparticle state change using external sources of energy. The comprehensive mechanism

established in this study allows for design and development of future targeted and controlled release of drugs using nanoparticles. 


\title{
How to design nanoparticles to aid in reversing drug resistant cancer using internal stimuli?
}

$>$ Designing nanostructures to genetically modify cancer for drug resensitization

\begin{abstract}
The current gold standard in drug delivery using liposomal nanoparticles that do not have active targeting capability, leading to higher dosage for required therapeutic effect. This also leads to acquired drug resistance by oncogene amplification and genetic mutations caused by cell survival activation pathways. In many cases the correlation between a gene amplification and resistance is not fully understood and requires a detailed study. To address this issue siRNA can be delivered, to end the mRNA production that causes specific protein amplification, but siRNA is very unstable and requires protection. Therefore, the aim of this study is to (1) develop a multifunctional nanoparticle that can be used to deliver siRNA in a protected environment as well deliver drug which can act on the resensitized cell and (2) make the nanoparticle targeted to the tumor to reduce off-side effects as well achieve lower effective dose. Particularly we chose to knockdown Axl pathway which has been shown to influence erlotinib TKI resistance in NSCLC treatment. Axl modulates various biological processes such as invasion, angiogenesis, resistance to chemotherapeutics and targeted drugs, survival and proliferation, thus believed to be playing a pivotal role in metastasizing cancers/malignancies. Separately to verify the role of AXL, CRISPR based gene editing was performed and subsequent effects were studied. This study will correlate Axl activation and expression with erlotinib sensitivity in NSCLC.
\end{abstract}




\section{METHODS}

In order to design a multifunctional protective nanocarrier, spherical biodegradable gelatin nanoparticles were examined for their siRNA loading efficiency, protection and delivery in multiple NSCLC cell lines. Antibody and siRNA encapsulation efficiency was performed using fluorescence spectroscopy. Cellular internalization of nanoparticles, delivery of siRNA was established using fluorescence microscopy. Furthermore, siRNA and crRNA assisted knockdown in cells were studied by monitoring protein expression using western blot, zymography, and mRNA expression using PCR. Finally, the results were associated with several NSCLC cell lines, biochemical pathways and microRNAs using KEGG bioinformatics. All aspects of knockdown were tested using erlotinib sensitivity tests performed through MTT, apoptosis, invasion and migration assays. Final validation was implemented in mice studies.

\section{RESULTS}

siRNA assisted knockdown and protein analysis revealed two oncogenes, AXL and FN14 that regulate erlotinib sensitivity in drug resistant NSCLC. Gene knockdown also confirmed the AXL-FN14 survival crosstalk. Further, targeted delivery of AXL/FN14 siRNA conjugated gelatin nanoparticles in mice led to reduction of tumors following erlotinib treatment. Overall this study demonstrates the stable delivery of siRNA using nanoparticles both in vitro and in vivo. 


\section{CHAPTER 4}

\subsection{INTRODUCTION}

In recent years, there have been large strides to understand drug efficacy post-treatment in cancer patients ${ }^{173-175}$. This heightened interest is primarily due to acquired drug resistance in patients ${ }^{176-178}$. The cause of these resistance cases are tied to poor understanding of posttreatment biochemistry. A major part of these biochemical issues partly tie with overexpressed oncogenes or mutations that compensate for the drug action in cancer cells $^{179-181}$. Frequently seen biomarkers in cancer patients include tyrosine kinase receptors families that are required for cell growth ${ }^{182-185}$. Predictably, the upregulation of these genes in cancer cells lead to uncontrolled growth of tumors. For example, epidermal growth factor receptor (EGFR), KRAS, HER-2 are few of these upregulated oncogenes ${ }^{186-188}$. To complicate this situation, not all of these upregulations in cancer patients are common ${ }^{88}$. Additionally, these genes can also exist as genetic or acquired mutations that can further change the overall function ${ }^{189-192}$. Therefore, a drug needs to target these wild-type biomarkers and be specific to mutations as well. Of all cancers, lung cancers are mostly affected due to the sheer number of mutations that vary from patient to patient ${ }^{193}$. Within lung cancer non-small cell lung cancer (NSCLC) accounts for almost $80 \%$ of the cases ${ }^{194}$. It is seen, almost $10 \%$ of NSCLC patients harbor EGFR mutations ${ }^{195}$. In addition to EGFR, other dominant mutations such as KRAS or ALK are also prevalent. Hence, efforts have been made to slowly bring personalized medicine to target the specific mutations. Of most drugs, tyrosine kinase inhibitors (TKI) are used to target these mutations ${ }^{196-199}$. However, as the cause of these mutations are themselves not understood, treatment decisions are also affected. This in-turn leads to disease relapse and decrease in overall survival ${ }^{200,201}$. Thus, 
there is a need to understand the pathways and signaling cross-talk between these mutations and their impact on targeted drugs. This understanding can help combat the rise of acquired resistance to drugs in cancer treatment.

\subsubsection{Lung cancer diagnosis and treatment}

Existing treatment regimens are decided after a tumor biopsy ${ }^{88}$. The cancer tissues taken from the patient are subject to variety of analysis. Specifically, IHC, FISH and PCR techniques are used to analyze the tissue ${ }^{63-65}$. Primarily, IHC staining reveals the overexpression grade of certain receptors on the cell surface. Further, FISH or PCR are used to confirm and quantify these biomarkers. In this way, tumor biopsy enables clinicians to find patient specific biomarkers. These biomarkers are usually overexpressed oncogenes or mutations that can be targeted by certain drugs. These drugs specifically bind and suppress the oncogene expression and thereby inhibiting its $\operatorname{action}^{199}$. By matching the drug to the mutation, the effectiveness of the treatment is increased. Furthermore, as the drug efficacy is increased it leads to shorter treatment times and increases the quality of life in patients. Primarily the first line and second line treatments in lung cancer cases include FDA approved cocktail of immunotherapy PDL1 checkpoint inhibitors with TKIs such as erlotinib or gefitinib ${ }^{202,203}$. Both these small molecule inhibitors bind to the intracellular domain of EGFR and specifically inhibits its downstream pathway 204,205 . Additionally, these drugs can bind and inhibit other tyrosine kinase receptors as well albeit with low specificity ${ }^{204}$. Both drugs target mutated EGFR that is very common in NSCLC patients. This specificity allows reduction in tumor growth and eventual control on the cancerous tissue. Although, the efficacy of 
these targeted therapies are very high at an initial stage, it is noticed that patient survival chances are still not correlative ${ }^{206}$. In recent years, there is a dramatic decrease in overall survival rate due to an increase in cases with treatment relapse. Much of the relapse has been tied to acquired drug resistance ${ }^{204}$. Due to resistance, the drug action often fails before the end of the treatment duration. Therefore, several studies are now underway to recognize the cause of resistance in hopes to increase the drug efficacy ${ }^{207-209}$. However, the underlying causes are still not understood.

\subsubsection{Acquired drug resistance to TKI}

TKI treatments often involve systemic use for $8-20$ months $^{195}$. Frequent use of the dug kills the tumor cells, but generates some cells that become immune to the drug. These TKI immune cells eventually grow to overtake the existing set of cancer cells and for resistant tumors. For example, patients treated with erlotinib often develop acquired resistance within 9-14 months ${ }^{195}$. The immunity to drug in these cells are established by forming resistant mutations that affect the drug action. Typically, TKIs bind to the intracellular ATP binding region of the EGFR receptor by competitive binding ${ }^{205}$. Studies have shown that these mutations may cause an increased affinity to ATP at the biding site by switching amino acids ${ }^{178}$. Another study also correlated increased ATP concentration in cells to increased competitive binding with erlotinib to the region ${ }^{204}$. This was reasoned due to ability of irreversible drugs such as CL-387785 that can still covalently bind and inhibit EGFR in resistant cells ${ }^{204}$. Overall, these mutational responses are a means of cancer cell survival. 
Several clinical studies have shown that, acquired resistance not only thwarts drug action but is increasingly correlated with worsening disease progression ${ }^{210-213}$. Recent studies have tied this increase in disease progression with invasive qualities of resistant cells ${ }^{79}$, ${ }^{214}$. Therefore, not only cells adopt new pathways to inhibit drug action, but simultaneously adopt invasive actions to quickly migrate and metastasize. Consequently, tumors spread and patient survival chances rapidly drop. This correlation is now being given serious importance as drug treatment are increasingly tied with exacerbated tumor spreading. A recent study on TKI inhibitors showed that the drug action might be disturbing normal cell regulation mechanisms ${ }^{215}$. These include regulation of receptor expression on the cell surface. In that study the authors argue that TKIs often target downstream pathways of EGFR such as MAPK/ERK or PI3K/AKT pathways. Although cancer cells often have uncontrolled growth mechanisms directly regulated by these downstream signaling proteins, the MAPK and AKT pathways are also used by intracellular mechanisms that continuously try to inhibit oncogene regulation. By targeting MAPK or MEK downstream, these regulatory mechanisms are impeded, causing a chain reaction of events leading to more uninhibited oncogenic actions. The study found that targeting MAPK pathways often led to deregulation of sheddase proteins such as ADAM 10 and 17 that shed receptors and control overall expression $^{215}$. Therefore, although cell growth was affected by targeting MAPK, the normal receptor shedding process was being stopped. Several studies have shown that decrease in sheddase activity in-turn increase receptor crowding on the surface ${ }^{78,215,216}$. This crowding enables a positive feedback loop to increase downstream action by autophosphorylation. Furthermore, this correlation is also evidently shown in in vivo 
studies $^{215}$. It is shown that, amount of receptors shed in blood of patients with resistant mutations are progressively low. The shed-receptor counts in the body fluid were inversely proportional to amount of receptors on the cancer cell surface. Studies indicated receptors such as AXL, MET or HER-2 are present in higher quantities due to deregulation of sheddase activity ${ }^{215}$. Interestingly, AXL upregulation is frequently seen in many resistant cancers ${ }^{82,217-219}$. Based on these studies, the AXL gene has been linked to TKI resistance.

\subsubsection{Role of AXL in TKI resistance}

AXL, that derives its name from the Greek word anexelekto that means uncontrolled, is present in most cells. The human AXL gene is located on chromosome 19q13.2 comprised of 20 exons. The structure of AXL contains two immunoglobin domains, two fibronectin type III, an extracellular proteolytic cleavage site, a transmembrane domain and a cytoplasmic domain that has the tyrosine kinase ${ }^{220}$. AXL belongs to class IX tyrosine kinase family and has documented to be upregulated in many cancers. Recently, AXL upregulations has been seen in patients undergoing treatment or with resistance ${ }^{82}$. In fact, most of the receptor overexpression seen in EGFR drug resistant cells belong to some tyrosine kinase family. These evidences suggest a tyrosine kinase cross-talk. AXL gene is responsible for encoding proteins related to growth, proliferation and antiinflammation in cells. Most importantly, AXL has been shown to activate the phosphoinositol-3 kinase signaling pathway downstream that is upregulated in almost all

cancers $^{221,222}$. In a separate study, AXL was also shown to activate MAPK/ERK pathway downstream $^{223}$. This information suggests, AXL upregulation due to sheddase 
deregulation during inhibitor treatment, might be back-activating downstream pathways to carry on the function of EGFR. Several studies have also shown that AXL is downregulated in cancer cells undergoing apoptosis ${ }^{222}$. Together, all these information suggests AXL may have an increasing role in causing resistance to EGFR inhibition by upregulating proliferative downstream signaling.

\subsubsection{AXL regulation of EMT}

Several studies have tied the proliferative abilities of AXL with activation of epithelial to mesenchymal transition (EMT) ${ }^{76,220,223,224}$. AXL upregulation in cells has also been correlated with increased expression of EMT proteins such as Vimentin, N-cadherin and MMPs. These EMT proteins change the morphology of the cancer cells to invade, migrate and proliferate ${ }^{225}$. This migration ability is gained by losing epithelial markers that are associated with cell-adhesion properties. The end result of upregulated EMT includes high motility of cells. The upregulation of EMT tied with AKT pathway can enhance cell proliferation and decrease cell apoptosis mechanisms ${ }^{222}$. Studies have shown a link between regulation of mTOR proteins and upregulated AKT pathways, wherein mTOR expression overrides normal cell-cycle regulatory pathways leading to uncontrolled $\operatorname{growth}^{226,227}$. All these downstream functions have been shown to be linked to NFkB that regulates gene expression within the nucleus ${ }^{227}$. Interestingly, these effects caused by EMT is commonly observed in patients with acquired resistance ${ }^{228}$. These evidence strongly supports the case for AXL's role in resistance causing mechanisms. However, little is known about this mechanism and warrants further understanding. Therefore, in this study, we hypothesize that, by inhibiting AXL we can possibly downregulate cell 
proliferation and resensitize cells back to EGFR treatment modality. To demonstrate the role of Axl in drug resistance, we used two approaches: (a) Downregulate AXL mRNA using siRNA delivery and understand the protein levels; and (b) Knockout Axl gene using CRISPR-CAS9 in drug resistant cancer cells and understand the cellular functions.

\subsubsection{Inhibition of AXL using siRNA (inhibitor therapy)}

Recent advances in mechanism of protein inhibition led to discovery of short interfering RNA (siRNA) ${ }^{229-232}$. siRNAs use RNAi based pathway that was evolved as an antiviral mechanism within mammalian cells. siRNAs have been shown to inhibit protein expression by impeding pathways specifically ${ }^{230}$. This specificity is achieved by base pairs in siRNAs that are complementary to the target mRNAs. siRNA act by forming a complex with an endoribonuclease DICER and Argonaute protein to form a RNAinduced silencing complex (RISC) ${ }^{233,234}$. Using the catalytic component of argonaute proteins the dicer can cleave and degrade mRNAs within the cell (Figure 101). Recent advances have made it possible to synthesize synthetic duplex siRNAs ${ }^{235}$. These siRNAs are around 19 base pairs and therefore small enough to be encapsulated or conjugated by adding additional functional groups to the $5^{\prime}$ end. Moreover, the length is short enough to evade interferon response when injected in the blood. Further, by adding UU overhangs these siRNAs have additional stability ${ }^{236}$. In addition, as dicer, argonaute and other adaptor proteins are naturally present in cells, the only functional component required for this process would be siRNA. Based on this information, by synthesizing an artificial siRNA complementary to AXL mRNA sequence we can thereby specifically 


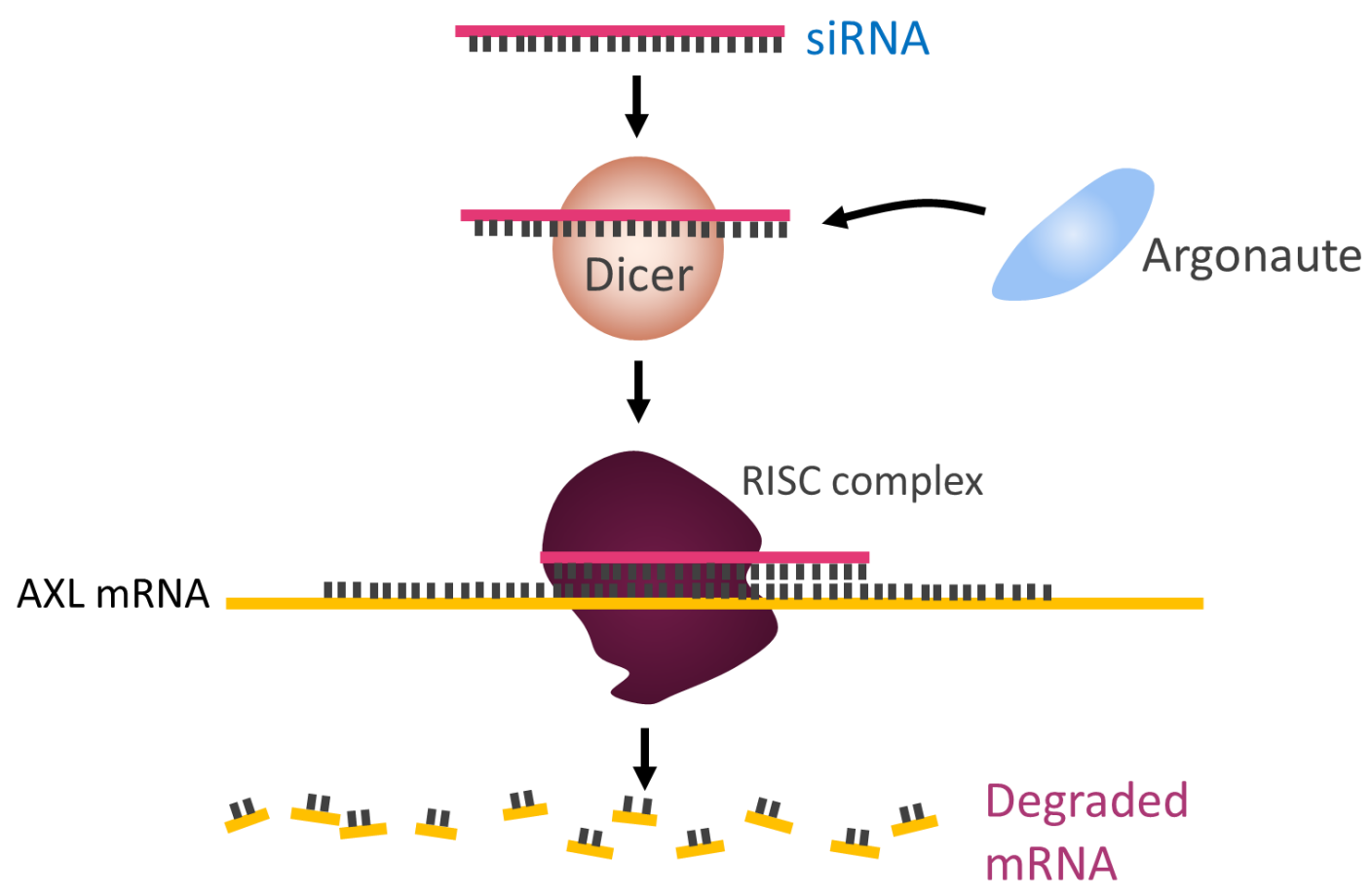

Figure 101. Activation of RISC complex using siRNA to degrade AXL mRNA in the cytoplasm.

target AXL within the cell cytoplasm. Therefore, for this study we used an siRNA (GGA ACU GCA UGC UGA AUG A) specific to the AXL mRNA within human cells ${ }^{237}$. This sequence is a short hairpin loop sequence and is very stable. Axl-siRNA was delivered using nanoparticle (covalent linking) and also by traditional transfection agent (electrostatic binding) and the mechanistic effects were studied in detail.

\subsubsection{Inhibition of AXL using crRNA (gene therapy)}

Recent advances in genome engineering has allowed highly specific DNA targeting systems such as RNA programmable clustered regularly interspaced short palindromic 


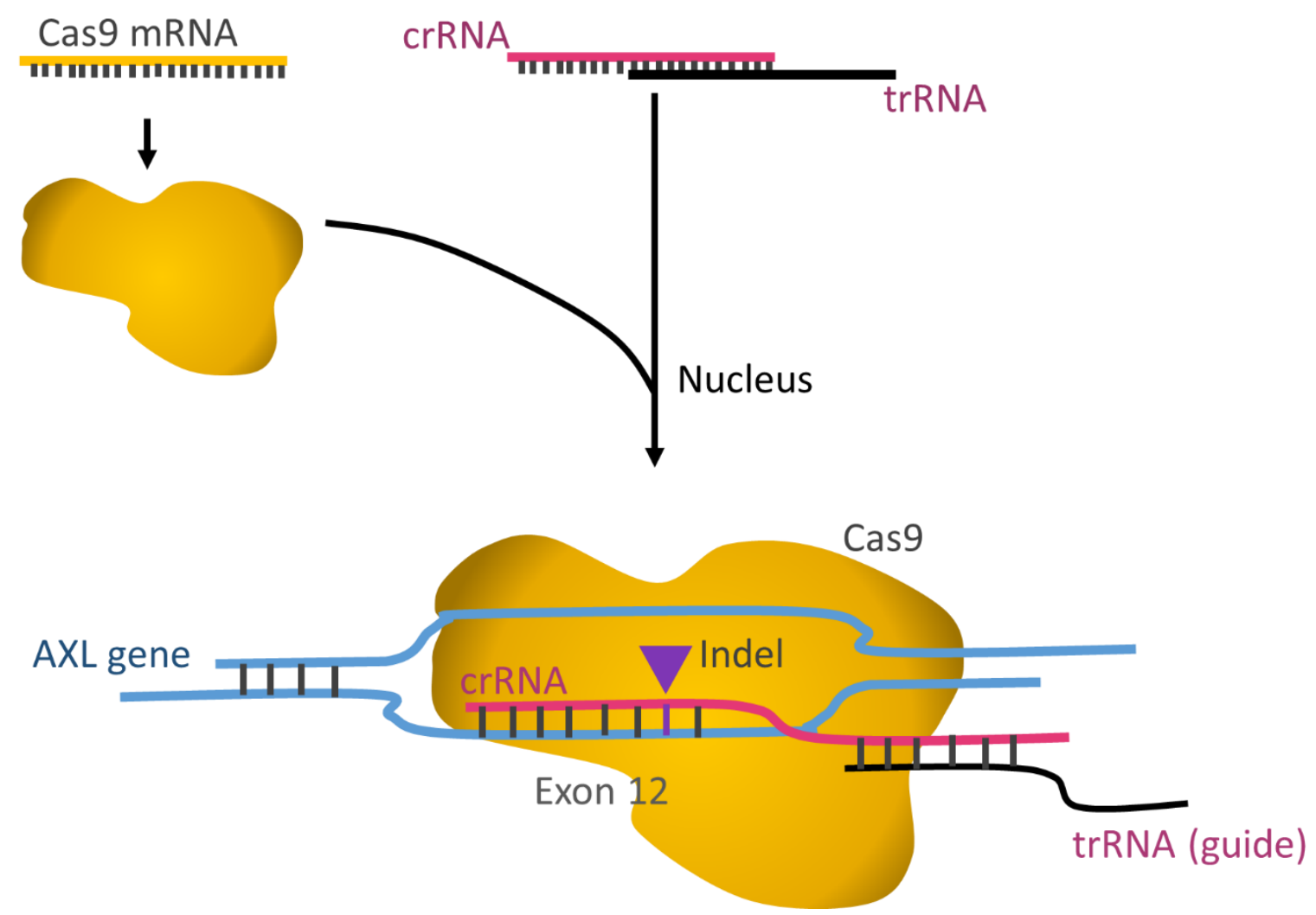

Figure 102. Formation of indel in the AXL gene using the complex formed by CRISPR RNA, Tracer guide RNA and the Cas9 endonuclease protein.

repeats (CRISPR)-Cas9 gene editing system ${ }^{238-240}$. Similar to RNAi pathway in higher organisms, the CRISPR system is also an evolved anti-viral cellular process found in bacteria $^{238}$. Several studies have shown that, using CRISPR-Cas9 highly site-specific indels can be introduced into target DNA ${ }^{241-243}$. The CRISPR functions using a CRISPRRNA (crRNA) that is complementary to the target DNA double-strand. Using a chimeric guide tracer-RNA (trRNA) the crRNA can associate itself with an endonuclease Cas9. Together, the complex can cause double-strand DNA nicks to cause a mutation within the genome (Figure 102). Once nicked, the DNA repair mechanism can then replace a nucleotide causing a mutation. However, as there will be a combination of mutations in 
different cells, this process would require careful selection of cell within a mutated population for selecting highly specific mutant alleles. Furthermore, as CRISPR system is of bacterial origin, it would require artificial production of Cas9 protein within the target human cells.

Comparable to synthetic siRNAs, crRNAs and trRNAs can also be synthetically produced. Additionally, Cas9 protein is genetically preserved universally functional endonuclease $^{239}$. Thus a synthetic mRNA would be capable of producing a functional protein within target cells. Therefore, for this study, we can use artificially synthesized AXL gene (exon 12) specific crRNA, synthetic trRNA, and synthetic cas9 mRNA to target the AXL gene and cause a mutation. This mutation will lead to a transcription of a non-functional mRNA, and a non-functional protein within the cell. Therefore, CRISPR induced AXL knockdown will inhibit the AXL downstream actions permanently. This allows for a careful genetic analysis of AXL actions before and after knockout within the cell.

\subsubsection{Translational medicine: Delivery of siRNA using nanoparticles}

Based on the available data and techniques we plan to knockdown the AXL functions and understand the basis of acquired resistance. However, studies have shown in vitro studies might not equate with in vivo studies ${ }^{244,245}$. Thus, it is necessary to test the hypothesis in higher organisms. For this purpose, the drugs and siRNA would have to be targeted to cancer tissues without off-target internalization within the organism. However, current practice often involves oral or systemic delivery of drugs that are nonselective. These drugs reach the tumor by enhanced permeability and retention (EPR) ${ }^{246-}$ 
${ }^{248}$. EPR effect is caused due to vascular permeability of tumors. The permeability is inturn due to the enhanced angiogenesis surrounding the tumor region required for the growth of tumors ${ }^{246}$. Therefore, due to the plentiful supply of blood vessels near the tumor, the probability of the drug reaching the tumor is very high. However, drugs often reach other organs using this method leading off-target side-effects and toxicity ${ }^{249}$. Therefore, in vivo studies would require development of an efficient cancer-targeted delivery agent.

The current Holy Grail in nanomedicine has been the selective delivery of inhibitors or targeting agents to the cytoplasm of cancer cells within the organism. To achieve this, different type of nanoparticles has been developed throughout this decade to target and traffic drug molecules in cancer cells $1,250,251$. Several studies have shown that nanoparticles are capable of diffusing through the tumor ${ }^{10}$. Because of size constraints, the particles accumulate and can theoretically increase drug concentration within the target. Additionally, several studies have shown that nanoparticles can be made porous for drugs to be encapsulated ${ }^{96,116,252}$. In addition to the porosity, nanoparticles can also be designed attach different targeting biomolecules (antibodies, peptides, aptamers and others) on the surface and encapsulation of drug molecules for targeted delivery 27,253 , ${ }^{254}$. However, delivery of siRNA has always met significant barriers ${ }^{255}$. This is mainly because, siRNA is easily subjected to degradation in bodily fluids due to presence of RNases $^{255}$. These endogenous enzymes are present not only inside the body, but present on surfaces outside as well. This makes siRNA a target for degradation in both environments. 


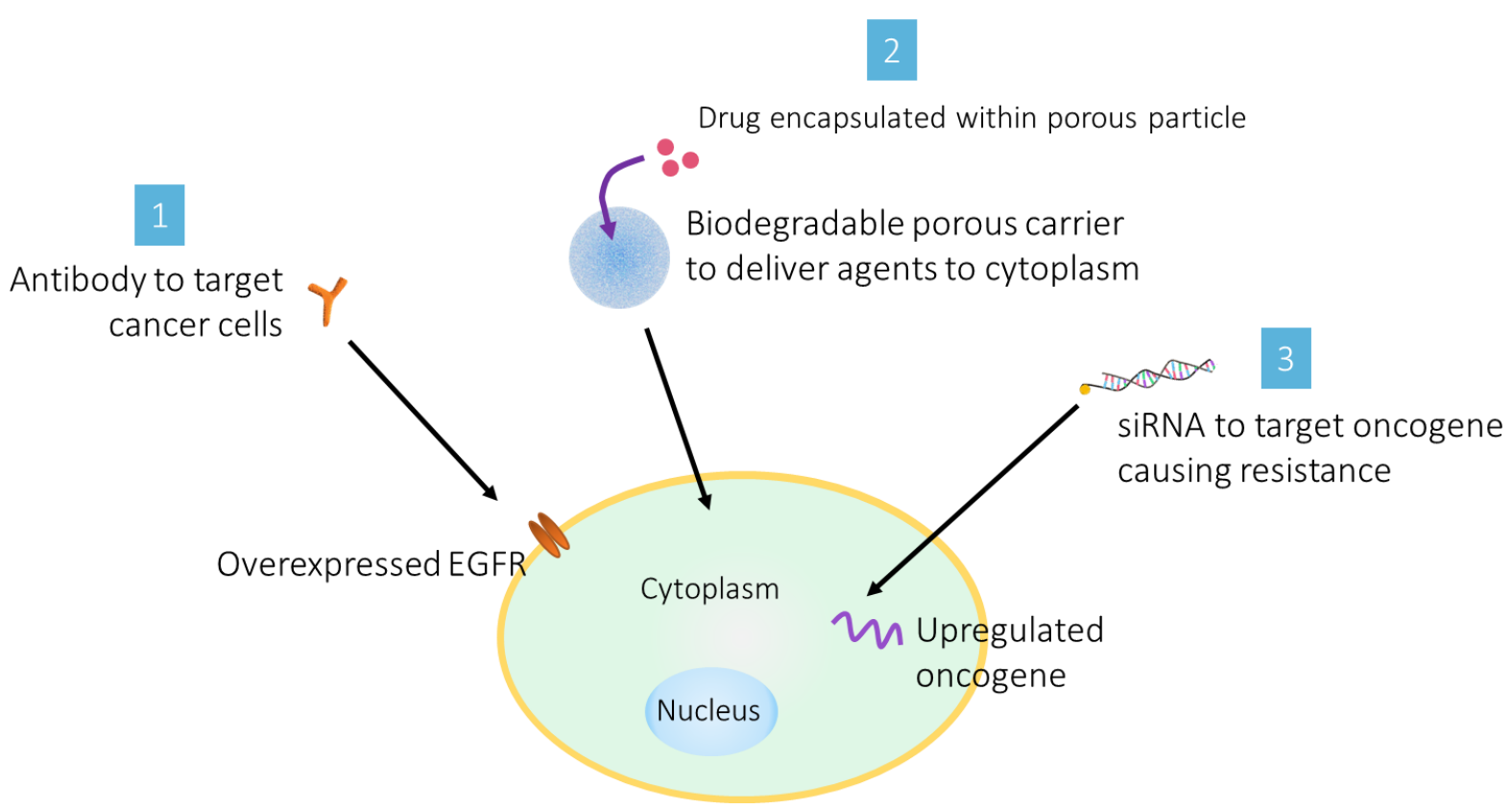

Figure 103. The three components needed to target, release drug and destroy resistance causing oncogenic mRNA within cell cytoplasm.

Additionally, siRNA being negatively charged, has an added disadvantage to cross the cell membrane due to repulsive forces. Therefore, majority of in vitro studies are performed using transfection agent. These agents exhibit toxicity at higher concentrations and cannot be used in vivo ${ }^{256}$. Hence, it is important to design a drug encapsulated nanoparticle that can protect and stabilize siRNA for both in vitro and in vivo environments. For this purpose, we designed a porous biodegradable protein based gelatin nanoparticle. Because of its biodegradable nature, the nanoparticle would be highly cytocompatible. The protein structure allows encapsulation of drug and surface functionalization using targeting antibodies/peptides. Additionally, the cross-linked nature of gelatin matrix can protect short strands of siRNA and thereby enabling siRNA delivery. In our study we functionalized an FDA approved anti-EGFR antibody 


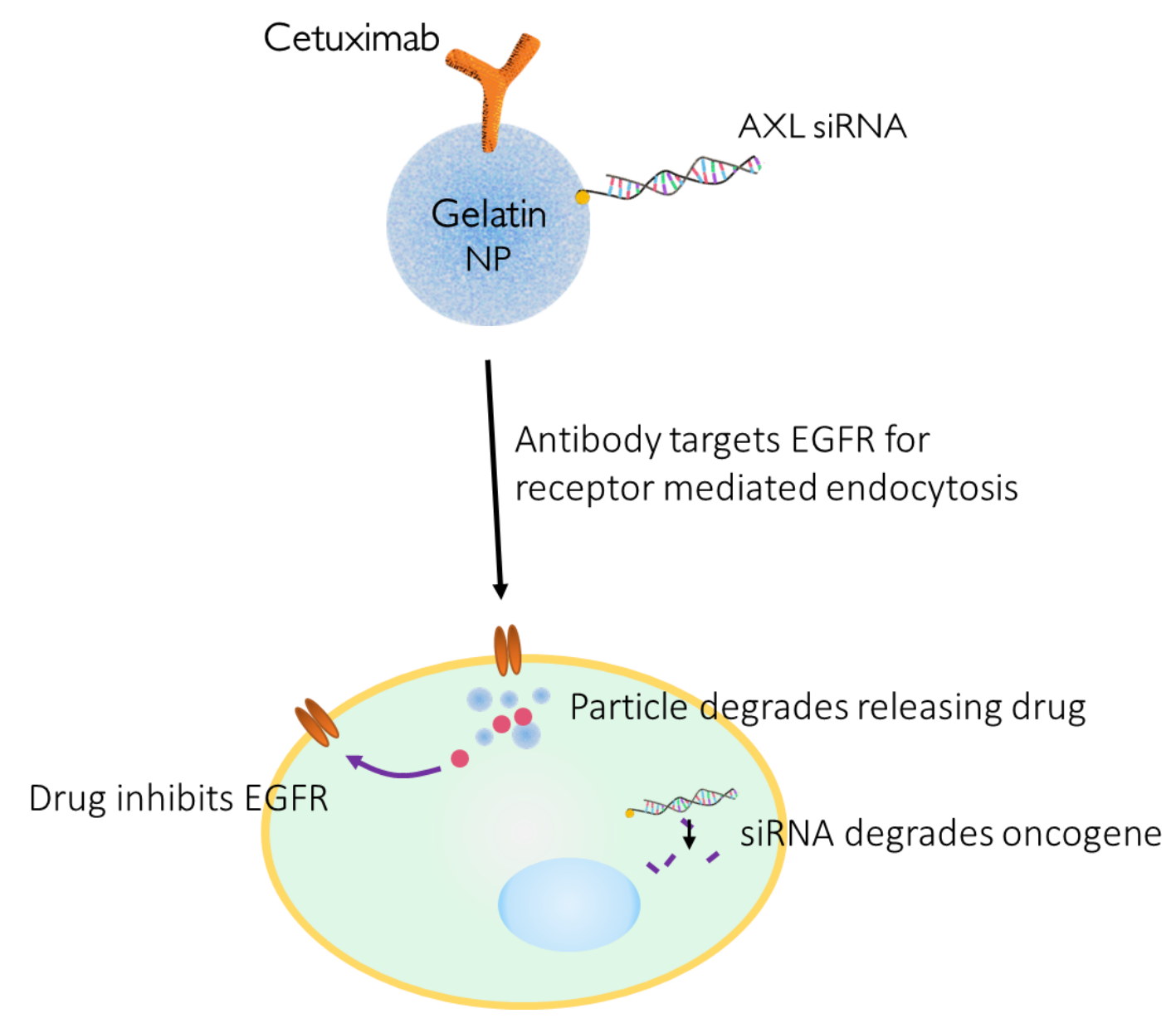

Figure 104. Design of an antibody conjugated porous drug encapsulated multifunctional nanoparticle with siRNA on its surface. These particles once internalized will degrade within the cytoplasm using proteases and release both drug and siRNA to block and inhibit EGFR pathway and AXL pathway repectively.

(Cetuximab) on the nanoparticle surface to target overexpressed EGFR receptors in cells. We hypothesize the large structure of antibody will give additional protection to short siRNA on the gelatin matrix. Furthermore, we utilized a custom modified siRNA 5' (AXL, FN14 and KRAS) end with a thiol group to conjugate to the gelatin nanoparticle. The thiol group was protected to avoid dimer formation. Therefore, we combined three components to form a multifunctional nanoparticle that can target, internalize, degrade to release drug, and release siRNA to downregulate AXL mRNA (Figure 103). This multifunctional particle should be able to internalize via receptor 
mediated endocytosis, while the gelatin protein would be degraded by proteases within the cell cytoplasm to deliver both drug and siRNA (Figure 104). As the siRNA is freely available within the cytoplasm, it would form RISC complexes to degrade intracellular AXL mRNA thereby downregulating AXL. The details of the in vitro and in vivo studies are presented.

\subsection{METHODS AND CHARACTERIZATION}

\subsubsection{Materials}

Gelatin (300 Bloom, Type A), Tween 20, N-(3-Dimethylaminopropyl)-N'ethylcarbodiimide hydrochloride (EDC), N-Hydroxysulfosuccinimide sodium salt (sulfoNHS), 25\% Glutaraldehyde solution (Grade I), 2-(N-morpholino)ethanesulfonic acid (MES), bovine serum albumin, MS-SAFE Protease and Phosphatase Inhibitor, triton X100 and phosphate buffer saline were purchased from Sigma-Aldrich. Hydrochloric acid, sodium bicarbonate, sodium hydroxide, sulfosuccinimidyl 4-(Nmaleimidomethyl)cyclohexane-1-carboxylate (sulfo-SMCC), 2-mercaptoethanol, Alexa Fluor 488 Annexin V/Dead Cell Apoptosis Kit, Fluorescein, Prolong gold antifade reagent with DAPI, Pierce ECL Western Blotting Substrate, Pierce BCA Protein Assay Kit, RNase AWAY Decontamination Reagent, Ambion RNAsecure Resuspension Solution, Ambion DEPC-treated water, UltraPure DNase/RNase-Free Distilled Water and acetone were purchased from Thermo-Fisher Scientific. MicroAmp Optical 96-Well Reaction Plate with Barcode, TaqMan RNA-to-Ct ${ }^{\mathrm{TM}}$ 1-Step Kit, TaqMan MicroRNA Reverse Transcription Kit, TaqMan® Universal Master Mix II (no UNG), B2M, RNU48, TaqMan gene expression assays for AXL (Hs01064444_m1), EGFR (Hs01076090_m1), 
TP53 (Hs01034249_m1) and MZF1 (Hs01051952_g1), TaqMan MicroRNA assay for miR-34a (000426), miR-432 (001026), miR-374 (000563) and miR548b (0002408) were also purchased from Thermo-Fisher Scientific. Sulfur group linked siRNAs namely, AXL siRNA, AXL siRNA-Cy5, FN14 siRNA, KRAS-G12S siRNA were custom synthesized from GE Healthcare Dharmacon Inc. Edit-R CRISPR-cas9 tracrRNA, Edit-R Cas9 Nuclease mRNA, Edit-R Human AXL crRNA and DharmaFECT Duo transfection agent were also purchased from GE Healthcare Dharmacon Inc. TransIT-X2 transfection agent was purchased from Mirus Bio LLC. Fluorometric $8 \mu \mathrm{m}$ 24-Well cell invasion assay with basement membrane and migration assay kits were purchased from Cell Biolabs Inc. 10x TBS, 10x Tris/Glycine/SDS buffer, coomasie brilliant blue R-250, zymogram development buffer, zymogram renaturation buffer, zymogram sample buffer, $10 \% 10$ well $50 \mu 1$ ready gelatin zymogram gel, 4-15\% 10-well $50 \mu$ ready Mini-Protean TGX, Precision Plus Protein Dual color standard ladder and supported nitrocellulose Membrane $(0.2 \mu \mathrm{m})$ were purchased from Biorad. Anti-rabbit IgG, HRP-linked Antibody, $\beta$-Actin, AXL, p-AXL, Vimentin, N-cadherin, E-cadherin, EGF, p-EGF, FN14, survivin, 4EBP1, p-4EBP1, p70S6K, p-p70S6K, caspase-3, PARP, ERK(1/2), p-ERK(1/2), AKT, p-AKT, FoXO3a, p-foxO3a, p-foxO1a, p-STAT3, p-STAT5, p-JAK2, p-Jun, TGF $\beta$, p-smad-3, smad4, Nucleolin and NFkB were purchased from Cell signaling technology. Cetuximab (Erbitux) was purchased from Eli Lilly and company. RNAse Free TE Buffer, pH 7.4 and RNAse Free 1 M Tris HCl, pH 7.4 were purchased from Boston BioProducts. Erlotinib was purchased from Selleck Chemicals. Amicon $0.5 \mathrm{ml} 10 \mathrm{kDa}$ regenerated cellulose Ultracel centrifugal filter units were purchased from Merck Millipore Ltd. FITC Annexin apoptosis kit was purchased from Abnova. Research grade Captisol (modified 
cyclodextrin) was purchased from Ligand. Taconic transit cage for mice, TTC ${ }^{\mathrm{TM}}$ with clear high profile lid was purchased from Taconic Biosciences Inc.

\subsubsection{Instrumentation}

Nanoparticle core-size measurements were performed using FEI Tecnai F30 Twin 300kV HR-TEM. Hydrodynamic size and zeta potential were measured using a Malvern Zetasaizer Nano-ZS. Syringe pump (KDS-200 series) was used during the synthesis process for controlled addition of reactants. Centrifugation was performed on a 5424 Eppendorf and refrigerated RC 6+ Sorvall centrifuge. Flow cytometry was performed on a Beckman Coulter CyAn ADP system. pH was measured using a SevenCompact Mettler Toledo $\mathrm{pH}$ meter equipped with an InLab Micro electrode. Fluorescence imaging, fluorescence assays and UV-vis absorption assays were performed on a Cytation 5 Cell imaging multi-mode reader. Gel electrophoresis was performed on a Bio-Rad Mini-Protean Tetra system and blots were transferred using a Genscript e-blot transfer system. Western blot and zymography gel imaging and acquisition was performed using Image Lab ver 5.2.1 software on a ChemiDoc XRS system from BioRad. Band densitometry analysis was performed on Image Studio ver 5.2. RT-PCR assays were performed on a 384-well compatible 7900HT Fast Real-Time PCR System. Flow cytometry was performed on BD FACS Calibur designed for fragile cell sorting. Mice imaging was performed on a Preclinical Optical/X-ray Xtreme in vivo Imaging System with a high speed X-ray head $(500 \mu \mathrm{A})$ (Bruker, MA). 


\subsection{3 siRNA and crRNA agents}

To inhibit protein expression of certain pathways siRNA was used to target and downregulate AXL, FN14 and KRAS mRNA. For this purpose AXL siRNA (Sense: 5' S-S.G.G.A.A.C.U.G.C.A.U.G.C.U.G.A.A.U.G.A.U.U 3'), FN14 siRNA (Sense: 5' SS.C.U.C.A.G.A.U.G.U.C.C.U.G.A.A.A.U.U.C.C.A.U.U 3'), and KRAS siRNA for G12S mutation (Sense: 5' S-S.C.A.G.C.U.A.A.U.U.C.A.G.A.A.U.C.A.U.U.U.U 3') was used. For estimation of conjugated siRNA and fluorescence imaging in vitro, Cy5 was conjugated to the anti-sense 3' end of AXL siRNA.

AXL crRNA was designed to target TTCAGTGGTCCGACGACTGT at genomic location and PAM: hg38|-chr19:41243678-41243700 AGG, in human NSCLC NCIH820 to generate AXL knockout cell-line (H820AK). The respective crRNA targets include Exon 12 (NM_021913), Exon 11(NM_001699) and Exon 9 (NM_001278599).

\subsubsection{Synthesis of Gelatin Nanoparticles}

Gelatin nanoparticles were synthesized using two step desolvation process ${ }^{257} .500 \mathrm{mg}$ of Gelatin type A was dissolved in $11 \mathrm{ml}$ of DI water in a $100 \mathrm{ml}$ beaker at $50{ }^{\circ} \mathrm{C}$ for 1 h (300 RPM). After complete dissolution, first step of desolvation using rapid addition of $20 \mathrm{ml}$ acetone was initiated for $30 \mathrm{~s}$. Low mol. wt. solids in the supernatant were discarded and the remaining white precipitate at the bottom of the flask was dissolved again in $12 \mathrm{ml}$ of DI water at $50{ }^{\circ} \mathrm{C}$ for $2 \mathrm{~h}(300 \mathrm{RPM})$. After complete dissolution, the solution was transferred to a $20 \mathrm{ml}$ glass vial and $\mathrm{pH}$ was adjusted to $2.8 \mathrm{using} 1 \mathrm{M} \mathrm{HCl}$. Now the solution was transferred into a $100 \mathrm{ml}$ round bottom flask and kept for stirring (300 RPM) in an oil bath at $50{ }^{\circ} \mathrm{C}$ for $1 \mathrm{~h}$. Subsequently the second step of desolvation 
was initiated by adding acetone drop wise $(100 \mathrm{ml} / \mathrm{h})$ using a $20 \mathrm{ml}$ syringe in a syringe pump. Once solution turned opaque white, addition of acetone was stopped. After 10 min, $200 \mu \mathrm{l}$ of $25 \%$ glutaraldehyde (cross-linker) was added to the RBF and the reaction was kept at $50{ }^{\circ} \mathrm{C}$ for $15 \mathrm{~h}(300 \mathrm{RPM})$. After $15 \mathrm{~h}$, solution turned pale yellow indicating cross-linked gelatin nanoparticles that was cooled to room temperature. $20 \mu 1$ of $1 \mathrm{M}$ Tris glycine solution was quickly added to solution to quench excess glutaraldehyde followed by immediate centrifugation $\left(40 \mathrm{~min}, 12^{\circ} \mathrm{C}\right.$ at $\left.20,000 \mathrm{~g}\right)$. Pellet was resuspended and washed 5 times in DI water. Final suspension was passed through a $0.45 \mu \mathrm{M}$ cellulose acetate filter and stored at $4{ }^{\circ} \mathrm{C}(5 \mathrm{mg} / \mathrm{ml})$. Non quenched particles were labeled as $\mathrm{G}_{\mathrm{NQ}}$ and quenched particles were labeled at $\mathrm{G}_{\mathrm{Q}}$. For all experiments $\mathrm{G}_{\mathrm{Q}}$ was used, unless indicated. For erlotinib encapsulated nanoparticles, $1 \mathrm{mg}$ of erlotinib dissolved in DMSO was added to the reaction solution prior to $\mathrm{pH}$ adjusting before the second desolvation step. These nanoparticles were labelled as (Er)G. In a similar fashion $1 \mathrm{mg}$ of fluorescein was dissolved during the synthesis step to yield fluorescein encapsulated gelatin nanoparticles labeled as $\mathrm{G}(\mathrm{Fl})$.

\subsubsection{Conjugation of antibody and siRNA}

$10 \mathrm{mg}$ of Gelatin nanoparticle solution was centrifuged and the pellet was washed with $1 \mathrm{ml}$ of $0.1 \mathrm{M}$ MES buffer ( $\mathrm{pH} 4.5$ ). Final pellet was resuspended in $0.5 \mathrm{ml}$ MES buffer in a $2 \mathrm{ml}$ eppendorf tube. To this tube, $200 \mu \mathrm{l}$ of $(10 \mathrm{mg} / \mathrm{ml}) \mathrm{EDC}$ and $300 \mu \mathrm{l}$ of (10 $\mathrm{mg} / \mathrm{ml}$ ) sulfo-NHS was quickly added and solution was kept in a shaker (850 RPM) for $3.5 \mathrm{~h}$ at $28{ }^{\circ} \mathrm{C}$. This allowed activation of carboxyl groups on the nanoparticle surface. Activated nanoparticles were centrifuged $(10,000 \mathrm{~g}$ for $10 \mathrm{~min})$ and the pellet was 
quickly resuspended in $600 \mu 1$ of $2 \mathrm{mg} / \mathrm{ml}$ Cetuximab (Ab) solution. Solution was kept in a shaker (800 RPM) for $15 \mathrm{~h}$ at $23{ }^{\circ} \mathrm{C}$ for conjugation of $\mathrm{Ab}$. Resulting reaction solution was then centrifuged (10,000 $\mathrm{g}$ for $10 \mathrm{~min})$ and the pellet was washed once with $1 \mathrm{ml}$ sterile $1 \mathrm{X}$ PBS. Final nanoparticle solution (in $1 \mathrm{X}$ PBS; $5 \mathrm{mg} / \mathrm{ml}$ ) was stored at 4 ${ }^{\circ} \mathrm{C}$. This reaction yielded two types of nanoconstructs labeled as GAb and (Er)Gab which were then used for siRNA functionalization.

$5 \mathrm{mg}$ of $\mathrm{GAb}$ or (Er)GAb solution in $1 \mathrm{X}$ PBS was taken in $1 \mathrm{ml}$ RNAse free microcentrifuge tube. To this $0.75 \mathrm{mg}$ of sulfo-SMCC dissolved in $200 \mu \mathrm{l}$ of RNAse free water was added. Reaction solution (pH 7.4) was kept in a shaker (800 RPM) for 2 h at $23{ }^{\circ} \mathrm{C}$. This allowed the NHS ester group of the SMCC crosslinker to covalently link with available amines on the nanoparticle as well as Ab. After reaction, solution was centrifuged $(10,000 \mathrm{~g}$ for $10 \mathrm{~min})$ and the pellet was resuspended in $600 \mu \mathrm{l}$ of RNAse free water. The $\mathrm{pH}$ of the resulting suspension was adjusted to 6.9 (electrode surface was cleaned with RNAse Away wipes prior use), and $50 \mu 1$ of $50 \mu \mathrm{M}$ thiol modified siRNA was added. Reaction solution was kept in shaker (650 RPM) for $2 \mathrm{~h}$ at $22{ }^{\circ} \mathrm{C}$. This allowed the sulfhydryl group of siRNA to covalently link to the free malemide group of the SMCC cross-linker on the nanoparticle surface. After reaction, solution was centrifuged $(10,000 \mathrm{~g}$ for $10 \mathrm{~min})$ and the pellet was washed with $1 \mathrm{ml}$ of RNAse free water to remove excess SMCC. Pellet was resuspended in $1 \mathrm{ml}$ sterile RNAse free water and $\mathrm{pH}$ was adjusted to 7.4. Final solution labelled as GAbsi or (Er)GAbsi was stored at $4{ }^{\circ} \mathrm{C}$. Depending on type of siRNA particles were labelled as GAbsiAXL or GAbsiFN14 or GAbsiKRAS for further experiments. For estimating the amount of siRNA conjugated, GAbsiAXL-Cy5 was utilized. For animal studies equal 
amounts $(25 \mu \mathrm{l}$ of $50 \mu \mathrm{M})$ of AXL and FN14 siRNA was reacted with gelatin nanoparticles. These particles were labelled GAbsi(AXL,FN14).

\subsubsection{Conjugation of antibody with NHS-Cy5 ester}

$300 \mu 1$ of $\mathrm{NaHCO}_{3}$ and $52 \mu 1$ of $10 \mathrm{mM} \mathrm{NaOH}$ was added to $1.2 \mathrm{ml}$ of Cetuximab $\mathrm{Ab}$ (2.4 mg). After adjusting $\mathrm{pH}$ to $8.5,79 \mu 1$ of Cy5-NHS ester ( $1 \mathrm{mg} / \mathrm{ml}$ in DMSO) was added to achieve a Ab:Cy5 ratio of 1:8. Reaction solution was kept in a shaker (800 RPM) for $2 \mathrm{~h}$ at $23{ }^{\circ} \mathrm{C}$ and then $13 \mathrm{~h}$ at $4{ }^{\circ} \mathrm{C}$. Final solution $(2 \mathrm{mg} / \mathrm{ml} \mathrm{Ab-Cy5})$ was used for conjugation with gelatin nanoparticles.

\subsubsection{Characterization of nanoconstructs}

For TEM imaging ( $80-120 \mathrm{kV}), 8 \mu \mathrm{l}$ of $0.1 \mathrm{mg} / \mathrm{ml}$ nanoparticle solution was air dried on CF300-Cu carbon film based copper grid. For hydrodynamic size and zeta potential measurements, $1 \mathrm{ml}$ of $0.1 \mathrm{mg} / \mathrm{ml}$ nanoparticle solution was used. To estimate amount of Ab conjugated, $\mathrm{Ab}-\mathrm{Cy} 5$ was used for fluorescence quantification. Fluorescence of the resulting supernatant from the reaction of GAb-Cy5 was used for measurements (ex 640 nm, em $665 \mathrm{~nm}$ ). Standard curve for Ab-Cy5 was used for calculations (Figure 105). Results show an average of 20-30\% Ab conjugation efficiency. Fluorescence of the resulting supernatant from the reaction of GAb-siAXL-Cy5 was used for 
a

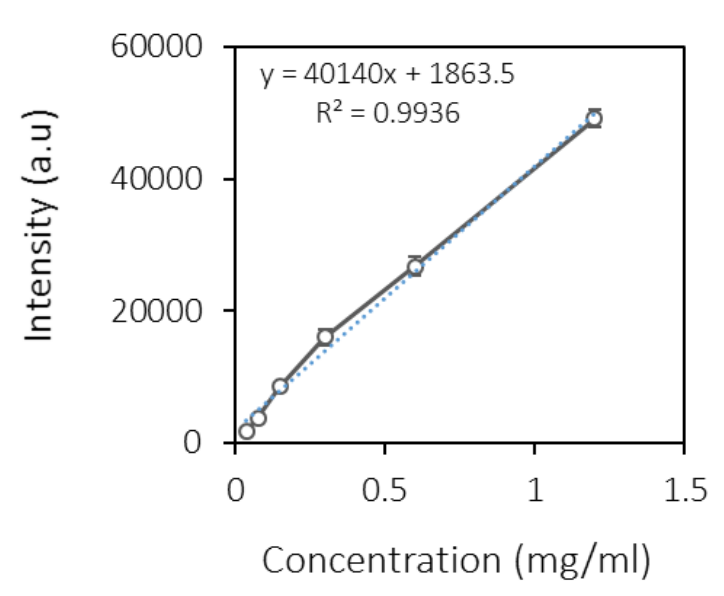

b

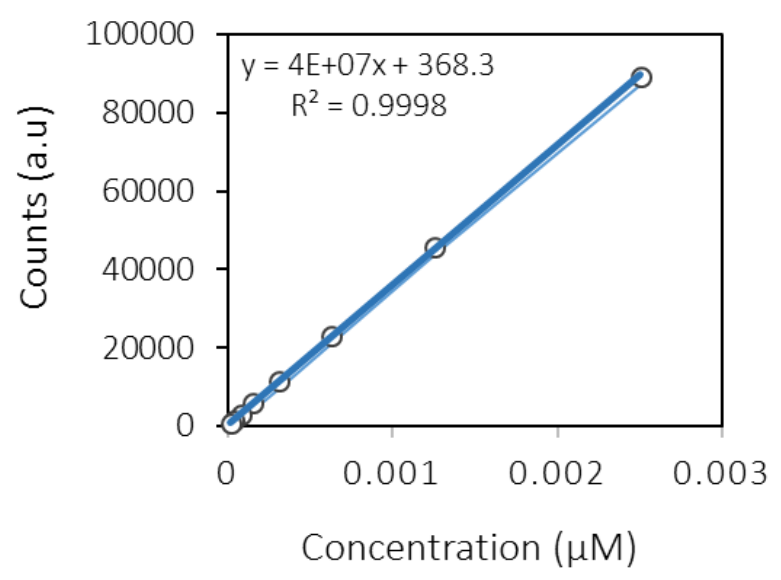

Figure 105. Standard curves for (a) Ab-Cy5 using Cetuximab and (b) siAXL-Cy5

(ex $640 \mathrm{~nm}$, em $665 \mathrm{~nm}$ ) estimating the amount of siRNA conjugation. Standard curve for siAXL-Cy5 was used for calculations (Figure 105). Results show an average of 9597\% siRNA conjugation efficiency.

\subsubsection{Cell culture}

HCC827 (E746_A750 del mut.), H1975 (L858R/T790M mut.) and A549 (KRAS G12S mut.) human non-small cell lung cancer cell lines (ATCC, USA) were grown in RPMI 1640 medium (obtained from Gibco BRL). The media was supplemented with $4.5 \mathrm{~g} / \mathrm{L}$ d-glucose, $25 \mathrm{mM}$ Hepes, $0.11 \mathrm{~g} / \mathrm{L}$ sodium pyruvate, $1.5 \mathrm{~g} / \mathrm{L}$ sodium bicarbonate, $2 \mathrm{mM}$ l-glutamine, $10 \%$ heat-inactivated fetal bovine serum (Atlanta Biologicals, USA), and 1\% penicillin/streptomycin antibiotic solution. H820 (E746_E749 del/T790M mut.) and H820AK (E746_E749 del/T790M/AXL mut.) cells lines utilized the same media supplements but with $20 \%$ heat-inactivated fetal bovine serum. Cells were cultured in a humidified atmosphere of $95 \%$ air and $5 \% \mathrm{CO}_{2}$ at $37{ }^{\circ} \mathrm{C}$ (Thermo Scientific, USA). 


\subsubsection{Fluorescence microscopy}

$5 \times 10^{5}$ Cells at $70 \%$ confluence were seeded on glass coverslips placed in 6-well plates and allowed to adhere overnight. Cells were treated with nanoparticles for $4 \mathrm{~h}$ followed by repeated washing and slides were preparation with DAPI nuclear stain. For visualizing gelatin NP, fluorescein encapsulated nanoparticles were used (ex $494 \mathrm{~nm}$, em $520 \mathrm{~nm}$ ). For visualizing antibody or siRNA, cy5 tagged molecules were used (ex $640 \mathrm{~nm}$, em $665 \mathrm{~nm}$ ). Slides were imaged at 40 and 60X using a Cytation5 imaging system.

\subsubsection{CRISPR for gene knockout cells}

$1 \times 10^{5} \mathrm{H} 820$ cells at $70 \%$ confluence were seeded in a 6 -well plate and allowed to adhere overnight. $25 \mu 1$ of crRNA-trRNA complex in RNAse free Tris-HCl buffer ( $5 \mu 1$ of 10 $\mu \mathrm{M}$ trRNA with $5 \mu \mathrm{l}$ of $10 \mu \mathrm{M}$ crRNA) was mixed with $20 \mu \mathrm{l}$ of $100 \mathrm{ng} / \mathrm{ul}$ cas9 mRNA, $100 \mu 1$ of Dharmafect duo transfection agent solution $(7 \mu 1$ of transfection agent with 93 $\mu 1$ of serum free RPMI media) and $55 \mu$ l of serum free media. This mixture was incubated for 20 min and then mixed with $800 \mu 1$ of 10\% FBS RPMI media to make up a total of $1 \mathrm{ml}$. Cells were washed 2 times with $1 \mathrm{X}$ PBS and the final crRNA+trRNA+Cas9mRNA solution was added to the well. Cells were incubated in 5\% $\mathrm{CO}_{2}$ at $37{ }^{\circ} \mathrm{C}$ for $72 \mathrm{~h}$. Up to this point all media used were devoid of antibiotics. After $72 \mathrm{~h}$, cells were washed with $1 \mathrm{X}$ PBS followed by incubation in cold TrypLE deattachment solution for $30 \mathrm{~s}$. Cells were gently dislodged using media and passaged in a T25 flask. Once cells achieved confluence, single cells were isolated and grown using serial dilution in a 96-well plate. Once these wells became 50\% confluent, cells were 
dislodged and transferred to a 6-well plate and in similar fashion to a T25 flask. Cells were collected and separately stored in cryogenic chamber for storage and preservation of gene knockout culture.

\subsubsection{1 mRNA and miRNA expression analysis using RT-PCR}

$1 \times 10^{6} \mathrm{H} 820$ and H820AK cells at 70\% confluence were seeded in 6-well plates. Cells were treated $(2.5 \mu \mathrm{M}$ erlotinib/ $1.25 \mathrm{mg} / \mathrm{ml}$ constructs/ $2.25 \mu \mathrm{M}$ siRNA) in serum free media for a period of $48 \mathrm{~h}$ followed by total RNA extraction. Untreated H820 cells were used as controls, and H820AK cells were not treated with any agents. Total RNA was isolated with the optional on column DNASE digestion step using the Qiagen RNASE free DNASE set (catalog number 79254) and miRNEASY Micro Kit (catalog number 217084). TaqMan microRNA assays (assay ID’s 000426, 001026, 000563, 0002408 representing mature microRNA sequences) were used to evaluate has-miR-34a, hasmiR-432, has-miR-374, and hsa-miR-548b. Total RNA (10 ng's from each sample) was reverse transcribed into complementary DNA (cDNA) using a TaqMan microRNA RT Kit. An additional run using $250 \mathrm{ng}$ 's of total RNA from each sample was completed to confirm results on has-miR-432 and miR-548b. Complementary DNA Products were combined with each assay, TAQMAN UNIVERSAL MMIX II (NO UNG), and then processed using a 7900HT Real-Time PCR System (Thermofisher). RQ manager was used to determine cycle thresholds with RNU48 serving as the endogenous control. TaqMan gene expression assays (assay ID's Hs01051952_g1, Hs01076090_m1, Hs01034249_m1, Hs01064444_m1) were used to evaluate MZF1, EGFR, AXL, and TP53.Total RNA (50 ng/ul from each sample) combined with each assay and reagents 
from the TaqMan RNA-TO-CT 1-STEP Kit was processed using a 7900HT Real-Time

PCR System (ThermoFisher). RQ manager was used to determine cycle thresholds with B2M serving as the endogenous control.

\subsubsection{Western blot}

1x10 $10^{6} \mathrm{H} 820, \mathrm{H} 1975$, A549 and H820AK cells at 70\% confluence were seeded in 6-well plates. Cells were treated ( $2.5 \mu \mathrm{M}$ erlotinib/ $1.25 \mathrm{mg} / \mathrm{ml}$ constructs/ $2.25 \mu \mathrm{M}$ siRNA) in serum free media for a period of $72 \mathrm{~h}$ followed by whole-cell lysate preparation using lysis buffer containing $1 \mathrm{X}$ phospho-protease inhibitor cocktail. Untreated H820, H820AK, H1975 or A549 cells were used as controls. Protein lysate was then centrifuged at $14000 \mathrm{~g}$ for $20 \mathrm{~min}$ at $4{ }^{\circ} \mathrm{C}$. Supernatant was analyzed for protein quantification and equalization using BCA assay. Proteins were then separated by 415\% TGX-PAGE and were transferred onto nitrocellulose membrane for protein blot analysis. Membranes were washed with 1X TBST, blocked with 5\% BSA blocking buffer containing $0.5 \%$ NGS for $2 \mathrm{~h}$ and incubated with primary antibody overnight on a shaker at $4{ }^{\circ} \mathrm{C}$. Blots were then washed before and after incubation with secondary antibody, and developed with chemiluminescence system.

For isolating proteins from mice tumor tissues, care was taken to surgically remove tumor without contamination of nearby muscle or epidermal tissue. Isolated tumor tissue was immediately snapped frozen in liquid nitrogen and stored at $-86^{\circ} \mathrm{C}$. Tumor tissue was homogenized to form a single cell suspension using metallic bead homogenizer in 1X RIPA buffer containing phospho-protease inhibitor cocktail. Tissue lysate was then centrifuged at $14000 \mathrm{~g}$ for $20 \mathrm{~min}$ at $4{ }^{\circ} \mathrm{C}$. Supernatant was analyzed for protein 
quantification and equalization using BCA assay. In order to remove impurities from the mice protein samples, acetone precipitation was performed. Briefly, to 1 part of tissue lysate, 4 parts of $100 \%$ acetone was added. Tubes were vortexed and kept at $-20{ }^{\circ} \mathrm{C}$ for 15 h. Precipitate was washed 3 times using $80 \%$ acetone in DI water by centrifugation $\left(14000 \mathrm{~g}, 20 \mathrm{~min}\right.$ at $\left.4{ }^{\circ} \mathrm{C}\right)$. At the final wash supernatant was removed, and the pellet was resuspended with $4 \mathrm{X}$ Lammeli buffer containing $\beta$-mercaptoethanol. Proteins were then separated using 4-15\% TGX-PAGE and were transferred onto nitrocellulose membrane similar to protein lysates described above. Blots were developed with chemiluminescence system.

\subsubsection{Invasion and migration assay}

$300 \mu 1$ of $1 \times 10^{6} \mathrm{H} 820$ and $\mathrm{H} 820 \mathrm{AK}$ cells at $70 \%$ confluence were placed inside the Assay inserts containing polycarbonate membrane. Treatment solutions $(1.25 \mathrm{mg} / \mathrm{ml}$ constructs/ $2.25 \mu \mathrm{M}$ siRNA) were directly added to the insert. $500 \mu 1$ of media containing $10 \%$ FBS was added to the lower wells to serve as attractant. Plates were incubated in $5 \% \mathrm{CO}_{2}$ at $37{ }^{\circ} \mathrm{C}$ for $24 \mathrm{~h}$ for the migration and $48 \mathrm{~h}$ for the invasion assays respectively. Insert media was aspirated and the insert was placed in a well containing detachment solution for $30 \mathrm{~min}$ at $37^{\circ} \mathrm{C}$. Cells were lysed and quantified using $4 \mathrm{X}$ lysis buffer/ CyQuant GR dye cocktail after a 20 min incubation at room temperature. Fluorescence measurements were performed on a 96 well plate (ex $480 \mathrm{~nm} / \mathrm{em} 520 \mathrm{~nm})$. Untreated H820 cells were used as controls, and H820AK cells were not treated with any agents. 


\subsubsection{Gelatin zymography}

$1 \times 10^{6} \mathrm{H} 820$ and H820AK cells at 70\% confluence were seeded in 6-well plates. After 8 h treatment $(2.5 \mu \mathrm{M}$ erlotinib/ $1.25 \mathrm{mg} / \mathrm{ml}$ constructs/ $2.25 \mu \mathrm{M}$ siRNA $)$ in serum free media, treatment media was removed and cells were washed multiple times with $1 \mathrm{X}$ PBS. Wells were replaced with $500 \mu \mathrm{l}$ of serum free media and incubated in $5 \% \mathrm{CO}_{2}$ at $37{ }^{\circ} \mathrm{C}$ for $20 \mathrm{~h}$. The cell-conditioned medium was then collected and centrifuged to remove cell debris at $4{ }^{\circ} \mathrm{C}$. Media was concentrated using a 10kd filter and equalized for protein concentration. Samples were mixed with equal volume of zymogram sample buffer and analyzed for MMP activity by using gelatin zymography run at $4{ }^{\circ} \mathrm{C}$. Gels were washed twice with DI water, twice with $100 \mathrm{ml} 1 \mathrm{X}$ renaturation buffer and once with $100 \mathrm{ml} 1 \mathrm{X}$ development buffer for $15 \mathrm{~min}$ on an orbital shaker. Gels were then incubated with $100 \mathrm{ml}$ of $1 \mathrm{X}$ development buffer containing $5 \mathrm{mM} \mathrm{CaCl}_{2}$ and $0.02 \%$ Brij-35 for $15 \mathrm{~h}$ at $37^{\circ} \mathrm{C}$ on an orbital shaker. Subsequently gels were washed, stained with coomassie blue $(0.5 \%)$ and destained (40\% methanol and $10 \%$ acetic acid) until clear bands were visible with good contrast. Gels were imaged using coomassie blue filter. Untreated H820 cells were used as controls, and H820AK cells were not treated with any agents.

\subsubsection{Apoptosis cell-cycle analysis}

The apoptotic progression of the cells induced by the constructs was determined with Flow cytometry analysis using FITC Annexin apoptosis assay kit (Abnova) according 
to manufacturer's protocol. Briefly, H820 cells at $70 \%$ confluence $\left(5 \times 10^{5}\right.$ cells/well $)$ were plated in 6 well plates for $15 \mathrm{~h}$ prior to treatment. For the treatment both siRNA and siRNA based constructs were used. The treatment was followed with and without drug treatment with untreated cells as control $(2.5 \mu \mathrm{M}$ erlotinib/ $1.25 \mathrm{mg} / \mathrm{ml}$ constructs/ $2.25 \mu \mathrm{M}$ siRNA). The viability and apoptotic rate of the cells were recorded for 48 and $72 \mathrm{~h}$ of treatment respectively. The cells were dislodged with TryplE and Enzyme Free Cells Dissociation Buffer (Life technologies) and washed with PBS once. Dislodged cells in media were kept at $37{ }^{\circ} \mathrm{C}\left(\mathrm{CO}_{2}\right.$ incubator $)$ to membrane restabilization for 15 min. Next, they were suspended in $1 \mathrm{ml}$ binding buffer. Annexin dye $(5 \mu 1 / \mathrm{per}$ test, $100 \mu \mathrm{g} / \mathrm{ml})$ and AAD stain $(1 \mu \mathrm{l} / \mathrm{ml})$ in $250 \mu \mathrm{l}$ Binding buffer were added and incubated

for 15 minutes. The cells were then washed once and re-suspended in $500 \mu$ l binding buffer. The cells were counted and sorted using a BD FACS Calibur Flow cytometer. The data was analyzed using BD FACS Diva 8.0 software and MS excel.

\subsubsection{MTT viability assay}

MTT tests were performed for all nanoconstructs treatments and controls for measuring $\mathrm{IC}_{50}$ for subsequent erlotinib treatment in H820, H820AK, HCCC827, H1975 and A549 cells $\left(5 \times 10^{5}\right.$ cells $\left./ \mathrm{ml}\right)$. Cells at $70 \%$ confluence were seeded in flat bottom 96 -well polystyrene coated plates for $12 \mathrm{~h}$ at $37^{\circ} \mathrm{C}$ in a $\mathrm{CO}_{2}$ incubator. siRNA with transfection agent in serum free media, and nanoconstructs in serum free media were prepared for control viability tests as well as treatments for erlotinib response. For TKI toxicity studies, siRNA $(2.25 \mu \mathrm{M} /$ well $)$ and nanoconstructs $\left(0.6 \mathrm{mgml}^{-1} /\right.$ well $)$ were initially treated in all wells for $4 \mathrm{~h}$. Then all wells were replaced with serum free media 
containing various concentrations of TKI for $72 \mathrm{~h}$ at $37{ }^{\circ} \mathrm{C}$ in a $\mathrm{CO}_{2}$ incubator. Each sample was tested in triplicates for statistical analysis. After $72 \mathrm{~h}$ incubation, $10 \mu \mathrm{L}$ per well MTT (stock solution $5 \mathrm{mg} / \mathrm{mL}$ 1× PBS) (ATCC, USA) was added and kept for $4 \mathrm{~h}$, and the formazan crystals so formed were dissolved in $100 \mu \mathrm{L}$ detergent/solubilizing buffer. The plates were kept for $2 \mathrm{~h}$ in the dark at $25^{\circ} \mathrm{C}$ to dissolve all crystals, and the intensity of developed color was measured using a Cytation 5 reader operating at $570 \mathrm{~nm}$ wavelength. Untreated cells were considered $100 \%$ viable. Intensities were then represented as dosage vs viability response plots.

\subsubsection{Generation of A549 luciferase tumor xenografts}

SCID-beige mice (C.B-Igh-1b/GbmsTac-Prkdc $c^{\text {scid }-L y s t^{b g}}$ N7) carrying double mutation for $\mathrm{T}$ and $\mathrm{B}$ lymphocyte defect (Age 5 weeks; male), were obtained from Taconic Biosciences, USA. All animals were housed in a pathogen-free environment under controlled conditions (temperature: $20-26^{\circ} \mathrm{C}$, humidity: $40-70 \%$, light-dark cycle: $12-12$ h). Chlorinated water and irradiated food were provided ad libitum. The animals were allowed to acclimatize and recover from shipping-related stress for one week prior to the study. The health of the mice was monitored daily. The protocol was reviewed by the Institutional Animal Care and Use Committee of the University of Notre Dame. Animal experiments were performed in accordance with the 'Guidelines for the Accommodation and Care of Laboratory Animals' of Institutional Animal Care and Use Committee (Protocol number 15-06-2586).

The immunodeficient mice were acclimatized and grown until 7 weeks before inoculation. Human A549-Fluc-Neo/iRFP-Puro Lung cancer cells were grown in 
complete medium and checked for contaminations. At $70 \%$ confluence, cells were washed with serum media to remove dead cells and washed with sterile 1X PBS. Cells were then harvested, counted and used for injections. A cell suspension of human A549Fluc-Neo/iRFP-Puro NSCLC cells $\left(3 \times 10^{6}\right.$ viable cells/mouse $)$ was subcutaneously inoculated into the right flank of each mouse. When the tumor grew to $150-200 \mathrm{~mm}^{3}$ (approximately 14 days), the mice were randomly divided into six groups of 5 mice each, and imaged to monitor the growth of tumor by fluorescence imaging.

\subsubsection{Mice treatment protocol}

Animals were administered nanoparticle construct GAbsi(AXL,FN14) intraperitoneally $(150 \mathrm{mg} / \mathrm{kg} \mathrm{BW})$, and intravenously $(25 \mathrm{mg} / \mathrm{kg} \mathrm{BW})$ at a dosing frequency of three times per week. Animals were also administered the nanoparticle construct GAbsi(AXL,FN14) intratumorly $(9.375 \mathrm{mg} / \mathrm{kg} \mathrm{BW})$ at a dosing frequency of once a week. As a therapy control group, animals were administered intravenously nanoparticle construct GAb without siRNA (25 mg/kg BW). All above animals and an additional independent group were orally gavaged freshly prepared Erlotinib in 6\% Captisol by at a dose of $15 \mathrm{mg} / \mathrm{kg} \mathrm{BW}$ every day until the end of therapy period. A vehicle control was administered saline $(0.2$ ml) by IV injections.

\subsubsection{Tumor imaging and analysis}

Animals were imaged for tumor growth on day 14, 17, and 20. Mice were injected IP $0.1 \mathrm{ml}$ of Redi Ject D-Luciferin ULTRA (Perkin Elmer, MA) and anesthetized using $2.5 \%$ isoflurane inhalation in a controlled vaporized tank. All images were taken on a 
4 MP Xtreme in vivo Imaging System. Mice were subjected to a $5 \mathrm{sec} 4 \times 4$ bin $180 \mathrm{~mm}$ FOV luminescence image as well as a $1.2 \mathrm{sec} \mathrm{X}$-ray image $45 \mathrm{kVp}, 180 \mathrm{~mm}$ FOV. Living image 2.5 software was used to determine the regions of interest (ROI) and average photon radiance $\left[\mathrm{p} / \mathrm{s} / \mathrm{cm}^{2} / \mathrm{sr}\right]$ was measured for each mouse. Luminescence-Xray overlay images were prepared with ImageJ v1.44p with LUT display. The total fluorescence was calculated by multiplying the mean fluorescence measured with area of observed fluorescence. For each mouse, the total intensity was then normalized with respect to the first day and the percentages were averaged from all mice cohorts.

\subsubsection{Statistics}

All statistics were performed using One-way Anova on Minitab. For animal studies difference in fluorescence was calculated between days and the values were used for statistical analysis.

\subsection{RESULTS AND DISCUSSIONS}

\subsubsection{Nanoparticle synthesis and characterization}

Gelatin nanoparticles were synthesized using a two-step desolvation process. Size analysis by TEM and DLS confirmed formation of uniform nanoparticles with a size of $\sim 200 \mathrm{~nm}$ (Figure 106 and Table 7). Nanoparticle formed during synthesis were crosslinked using the $\varepsilon$-amino residues available in the gelatin backbone. 


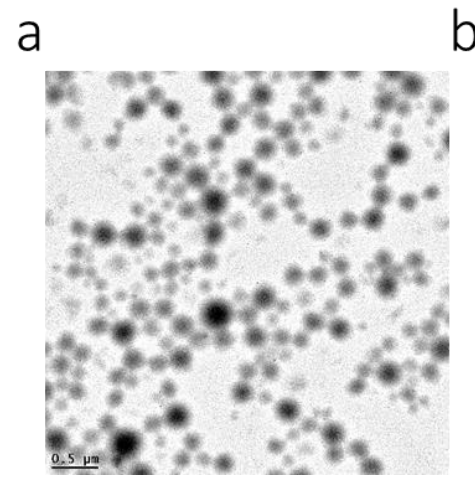

b
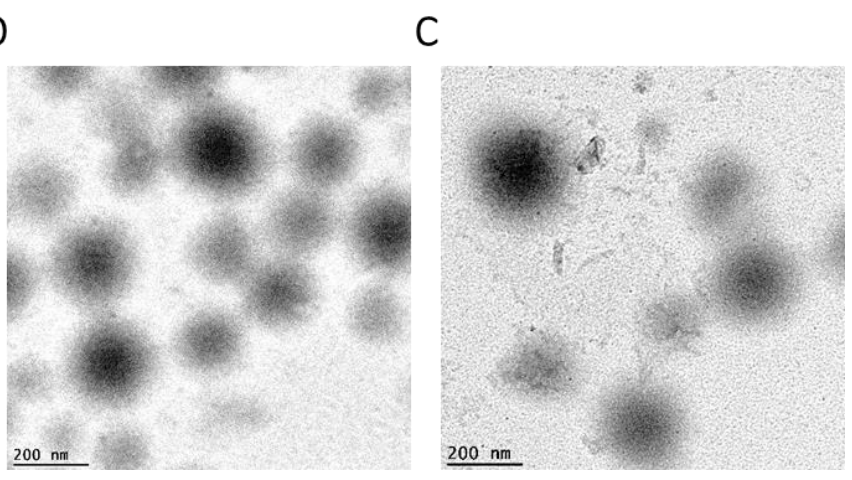

Figure 106. TEM images for (a) Gelatin nanoparticle (G) (b) antibody conjugated GAb and (c) antibody and siRNA conjugated GAbsiAXL.

An earlier study has shown the cross-linking efficiency to be around $60 \%{ }^{258}$. The carboxyl groups on the surface of gelatin nanoparticles were activated and conjugated with EGFR targeting antibody 259,260 . Fluorescence based estimation, indicated a $20 \%$ conjugation efficiency. The rest of available amine groups on the surface of gelatin as well as antibody were functionalized to NHS-ester of the SMCC cross-linker ${ }^{261}$.

\begin{tabular}{lll}
\hline Construct Name & Hydrodynamic size $($ d.nm $)$ & Zeta $(\mathrm{mV})$ \\
\hline Gelatin & 227 & 16 \\
\hline GAb & 261 & -9 \\
\hline GAbsiAXL & 228 & -28 \\
\hline
\end{tabular}

Table 7. Physicochemical properties of gelatin NP constructs. 
The malemide end of this cross-linker was used to conjugate the siRNA. To increase the cross-reactivity of the functional groups we modified an existing protocol ${ }^{257}$. The modified process decreased the reaction time to $2 \mathrm{~h}$ from $15 \mathrm{~h}$, and improved the conjugation efficiency and stability of siRNA. Results from fluorescence based quantification revealed a very high conjugation efficiency up to $98 \%$.

The changes in zeta potential upon antibody and siRNA conjugation confirmed the formation of the respective conjugates (Table 7). The stability of the conjugates was monitored by measuring the zeta potential and hydrodynamic size in biologically relevant media at different time points. Both measurements indicate that high stability for over a week period. (Figure 107a and 107b).

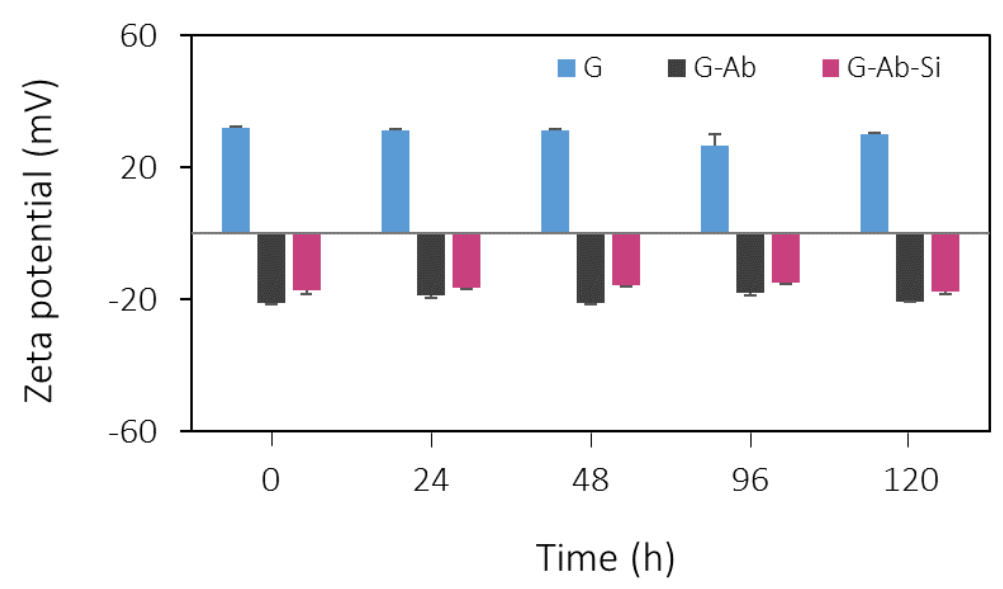

Figure 107a. Stability of Gelatin nanoparticle constructs evaluated through change in surface potential over time. 

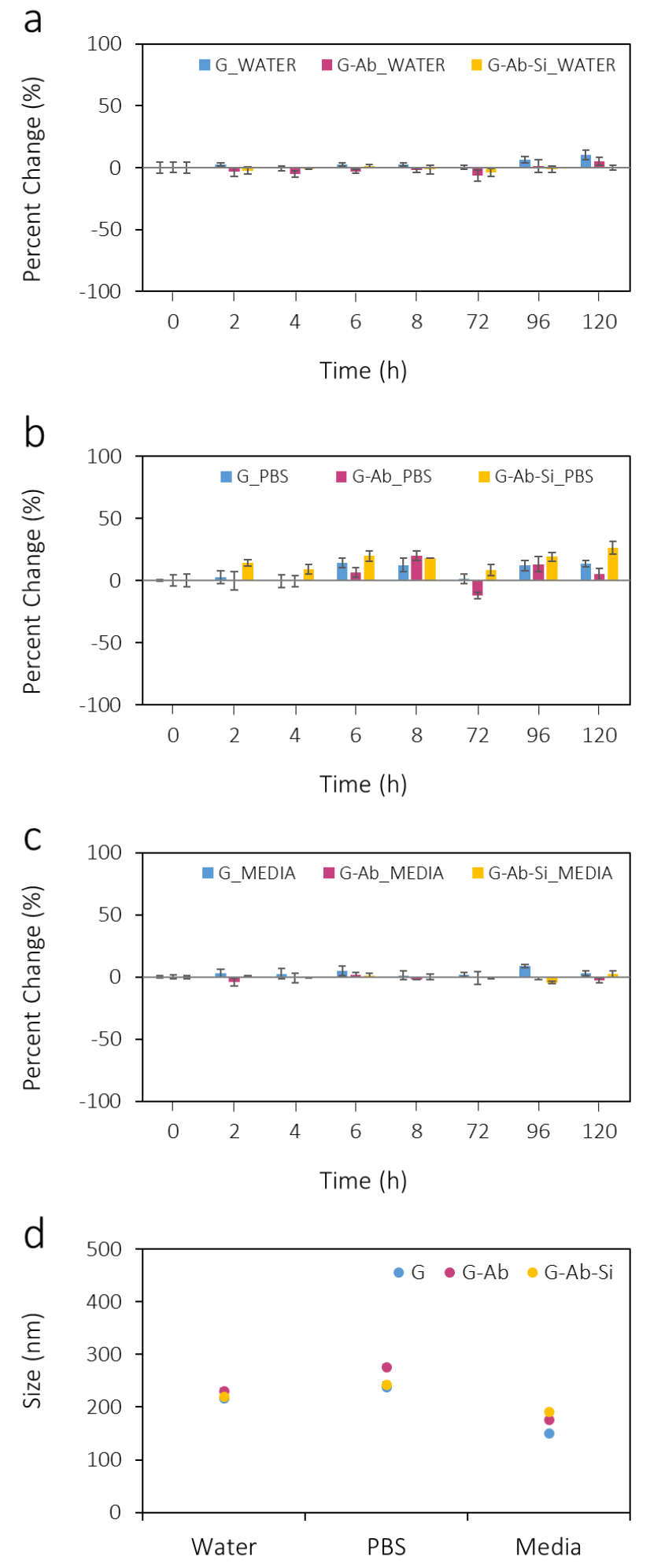

Figure 107b. Stability of Gelatin nanoparticle constructs evaluated through change in hydrodynamic size over time in (a) water, (b) 1X PBS, (c) RPMI media. Averaged results are shown in (d). 


\subsubsection{Nanoparticle targeting and delivery}

In order to test the ability of the antibody conjugated gelatin nanoparticles to target cancer cells, we evaluated the receptor-mediated endocytosis of nanoconstructs in multiple NSCLC cells in vitro. For this study, particles were allowed to internalize in cells and endocytosis was analyzed using the fluorescent-labelled antibody.
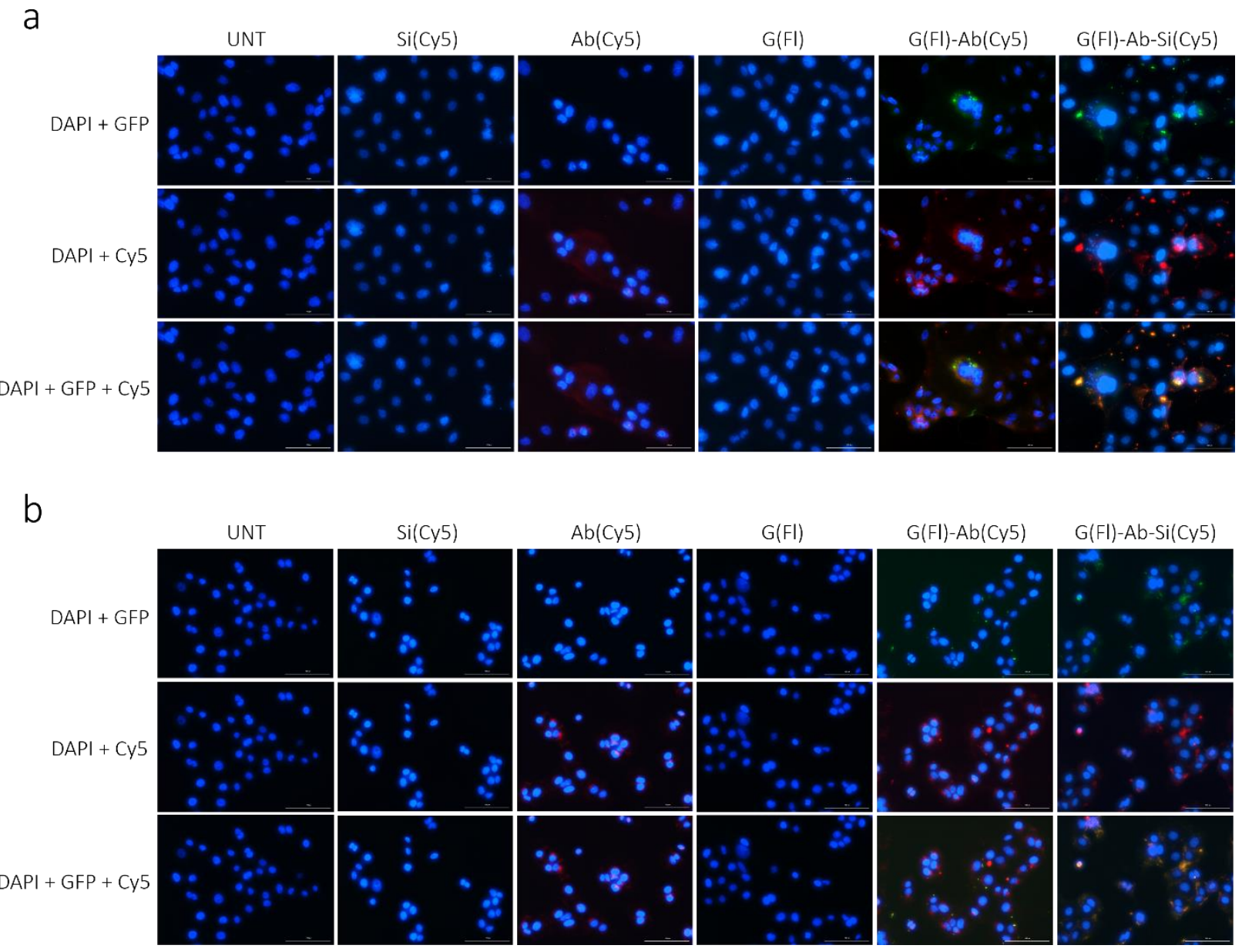

Figure 108. Internalization of targeted constructs in (a) H820 and (b) HCC827 cells (40x). Results indicate gelatin particles (Fluorescein) are only internalized when antibody is present on surface. Similarly, siRNA is internalized when gelatin particles are internalized by antibody targeting. 

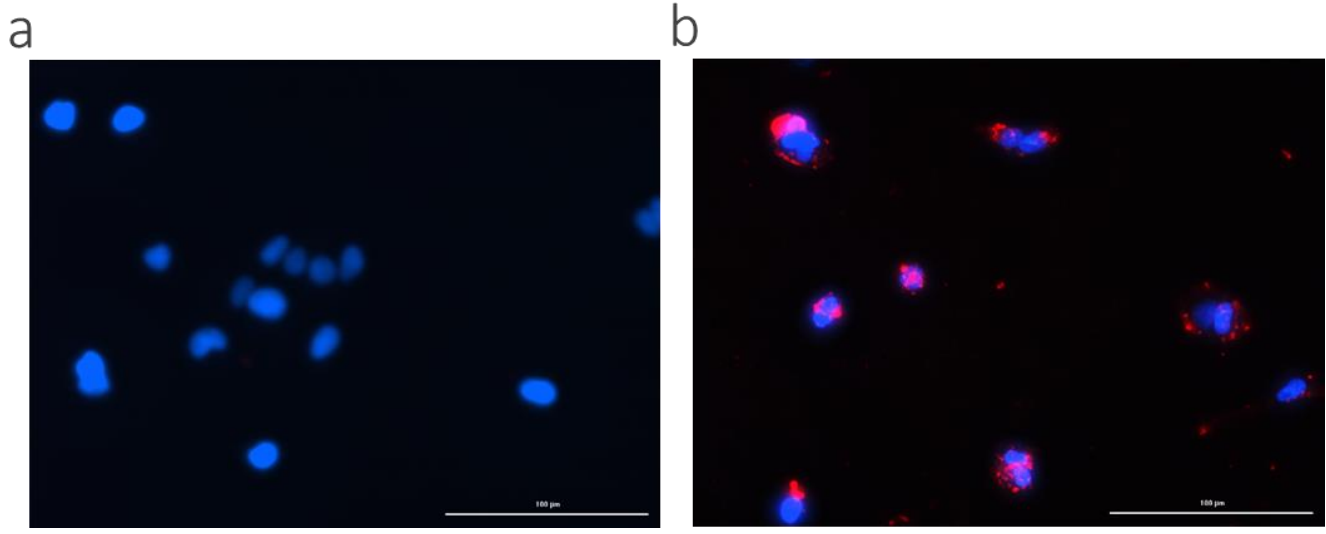

Figure 109. Internalization of targeted constructs in HCC827 cells: (a) GAb (b) GabsiAXL-Cy5 (60x). Results indicate presence of high quantities of siRNA (Cy5) in the cytoplasm of cells after internalization of construct.

Fluorescence microscopy images confirm internalization of GAb and GAbsiRNA conjugates through EGFR antibody in spite of its negative surface potential (Figure 108 and Table 7). As predicted, when cells were treated with nanoparticles without antibody no internalization was observed. Similarly, we also studied the internalization of siRNA using fluorescent tag. Fluorescence microscopy images show that siRNA internalized in very high quantities within the cytoplasm when antibody targeted particles were used (Figure 108 and Figure 109). The fluorescent data validates the targeting and siRNA delivering capabilities of nanoconjugates within the cytoplasm of the cells. An earlier study also confirmed the nature this endocytosis process and the presence of nanoparticle within the cytoplasm ${ }^{262}$. The results also suggests that the nanoparticle are very effective in transporting in siRNA to the cytoplasm without any degradation ${ }^{257}$. As majority of RNA transport occur near endoplasmic reticulum within the cytoplasm, we anticipate that the delivered siRNA should be able to knockdown the pathway very effectively in cells. 


\subsubsection{Downregulation of AXL using siRNA and generation of knockout cell line}

In order to investigate the role of AXL in drug resistance, as a first step we downregulated Axl and studied the related pathways. Earlier studies have shown that siRNA play an important role in post transcriptional gene silencing within the cell by utilizing the RNAi pathways ${ }^{232}$. Therefore, we used siRNA specific to target gene sequence, to silence and degrade the AXL mRNA within the cell. siRNA was first tested in NSCLC cells to evaluate downregulation of pathways.

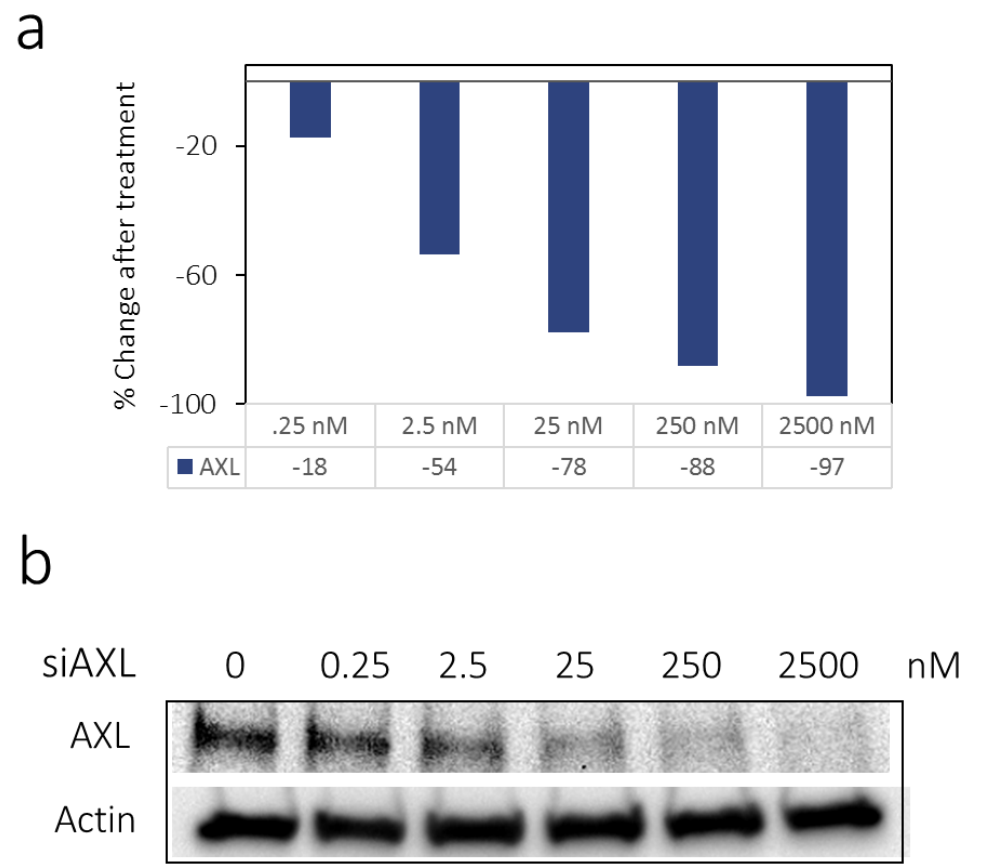

Figure 110. Downregulation of AXL using siRNA in H820 cells: (a) Band densitometry analysis (b) associated blot. 


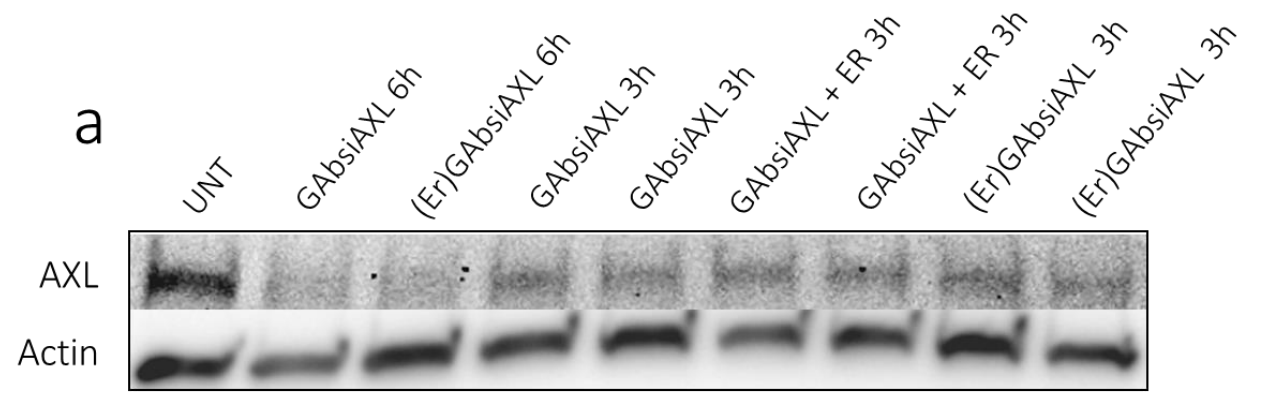

b
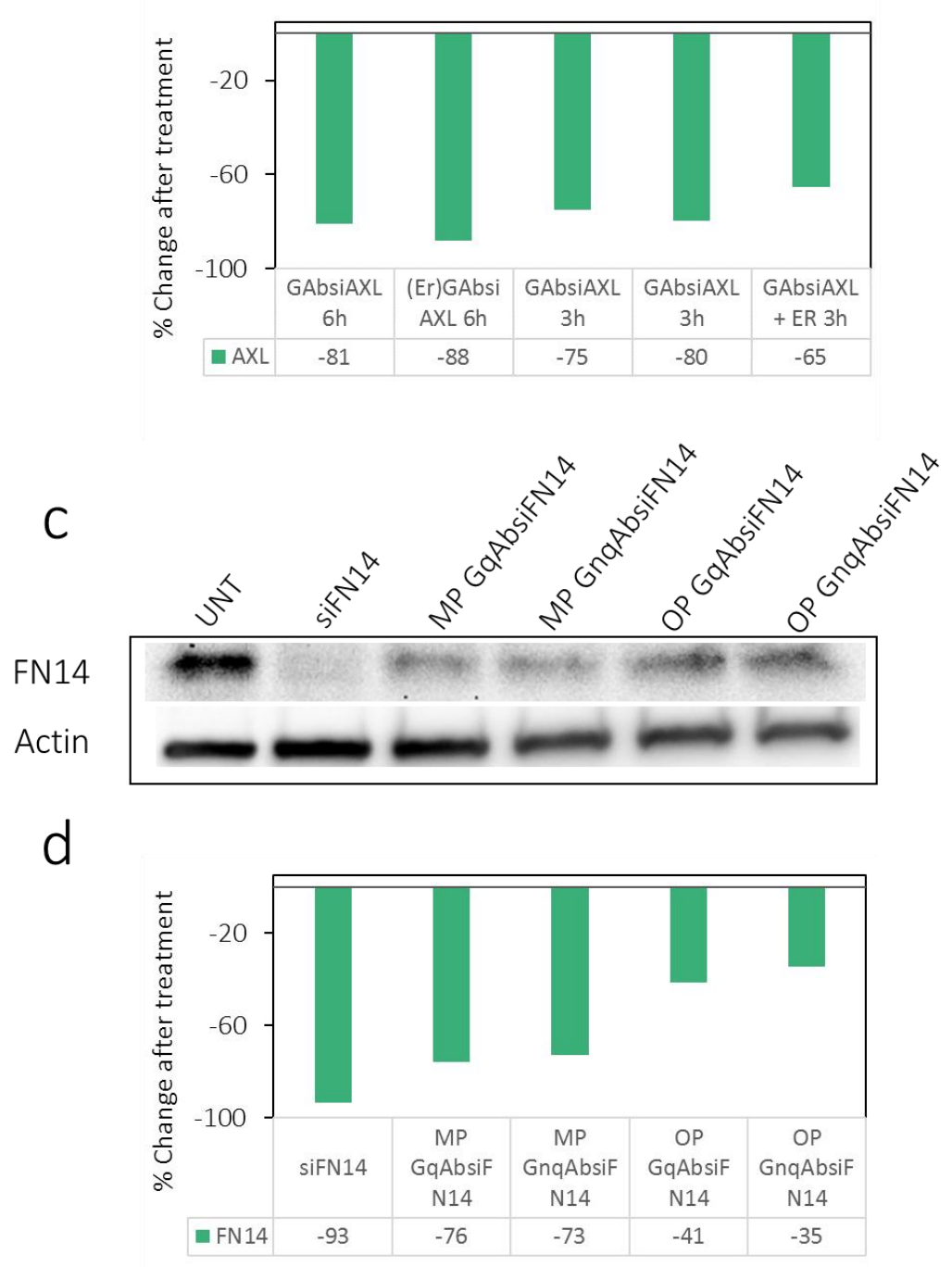

Figure 111. Downregulation of AXL using siRNA conjugated constructs in H820 cells: (a) associated blot (b) Band densitometry analysis. Downregulation of FN14 using siRNA conjugated constructs in H820 cells: (a) associated blot (b) Band densitometry analysis. MP and OP stand for Modified protocol and Old protocol respectively. Gq and Gnq stand for gelatin particles with glutaraldehyde quenched and non-quenched respectively. 


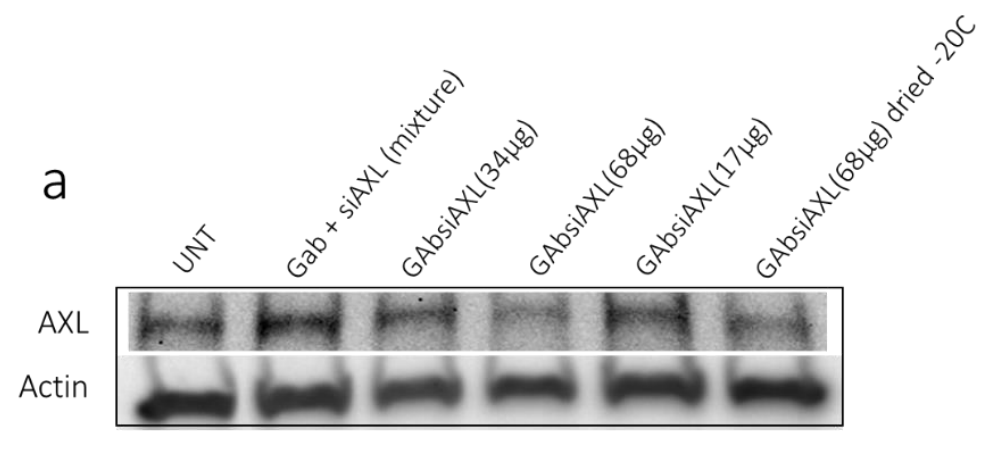

$\mathrm{b}$

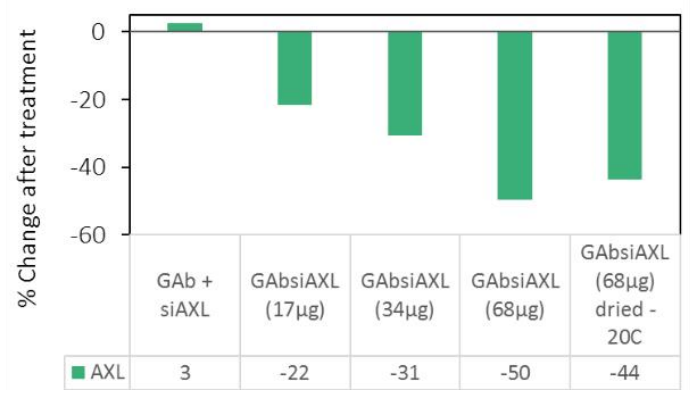

Figure 112. Downregulation of AXL using siRNA conjugated constructs in H820 cells: (a) associated blot (b) Band densitometry analysis. Results indicate that physical mixture of GAb and siRNA does not allow downregulation. Results also show that storing lyophilized power at $-20{ }^{\circ} \mathrm{C}$ does not adversely affect function of construct.

For this study, AXL siRNA was delivered into cells using transfection agent and the amount of siRNA need to achieve a high downregulation was estimated. Results indicate a concertation of $0.0025 \mathrm{mM}$ was sufficient to achieve a knockdown efficiency of up to $97 \%$ in cells (Figure 110). This siRNA concentration was chosen for conjugation and optimization strategies. To study the knockdown capabilities of siRNA by the internalized nanoparticles, we investigated the protein expression in H820 drug resistant cancer cells. For this study, we used multiple siRNAs that target different pathways such as AXL and FN14 within the cell. Results showed a very high knockdown efficiency achieved by the constructs as compared to controls (Figure 111). Additionally, the modified protocol had a higher knockdown efficiency (Figure 111). 


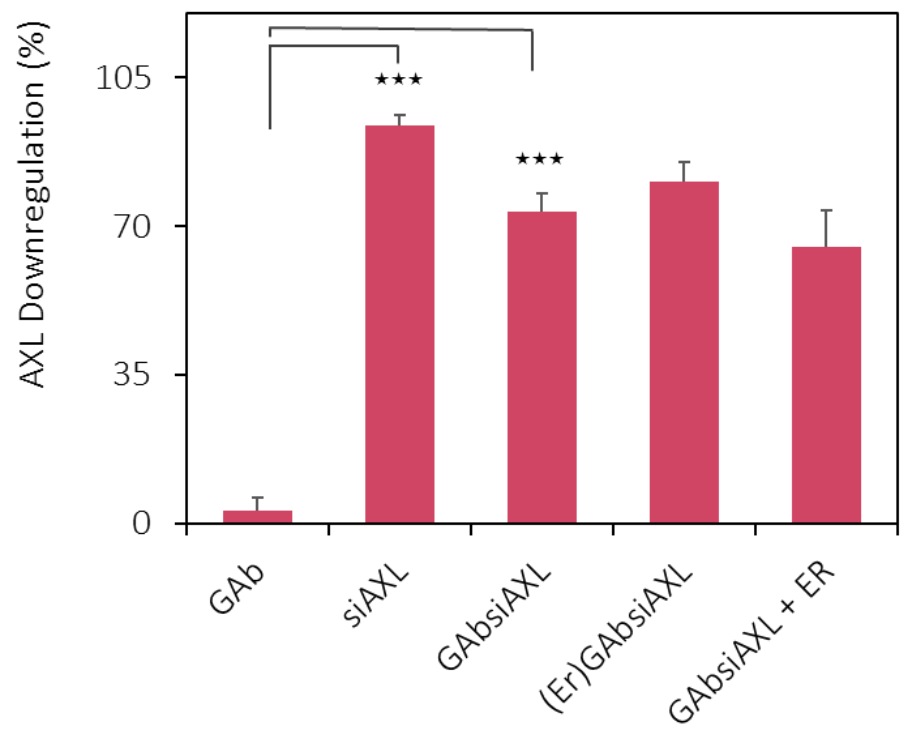

Figure 113. Cumulative analysis of AXL downregulation using siRNA alone, siRNA conjugated constructs and synergistic treatment of GAbsiAXL with erlotinib in H820 cells. Results show siRNA was efficiently downregulating AXL when present in sample. These results suggest nanoparticle based delivery of siRNA was highly stable and efficient. As shown in figure GAb control did not affect AXL in cells. ${ }^{\star \star} \mathrm{p} \leq 0.001$, and the values were analyzed using one-way anova

The lyophilized siRNA conjugated particles (stored at $-20{ }^{\circ} \mathrm{C}$ for an extended period of time) were stable and effective in silencing AXL mRNA (Figure 112). Overall, these results also indicate very high siRNA stability post conjugation with gelatin nanoparticles (Figure 113). Based on these experiments, we can efficiently target specific mRNA within the cell and target downstream activity of proteins.

Although blocking through siRNA was efficient, the mRNA is continuously produced within these cells and the activity of siRNA wear off after $72 \mathrm{~h}^{263}$. Thus, an effective therapy based on siRNA would require frequent administration of siRNA to stop the protein translation activity. To work around these limitation, we directly targeted the functionality of the gene responsible for mRNA transcription. By this 
alternative method, a certain protein responsible for regulating oncogenic pathways could be permanently disabled. A recent study demonstrated such a selective gene knockdown technique using clustered regularly interspace short palindromic repeats (CRISPR) ${ }^{239}$. We used crRNA (CRISPR) for knocking down the AXL gene within H820 cells to generate AXL knockdown cells (H820AK). This will allow the AXL gene to be mutated permanently and produce a non-functioning AXL mRNA. The translation of these non-functional mRNA will result in proteins that will consequently stop the functionality of the pathway. In our study, we artificially transfected the Cas9 mRNA into cells and allowed cas9 protein to be translated in the cytoplasm. We simultaneously transfected a trRNA-crRNA complex (guideRNA-CRISPR) that associates with the cas9 protein to cleave the AXL gene fragment at exon 12. Indeed, we see a reduction of AXL protein synthesis within these cells after the gene was knocked down in H820 lung cancer cells (Figure 114). To further evaluate the gene expression of both siRNA and crRNA knockdown cells, we performed RT-PCR analysis to cross examine with our protein analysis. 


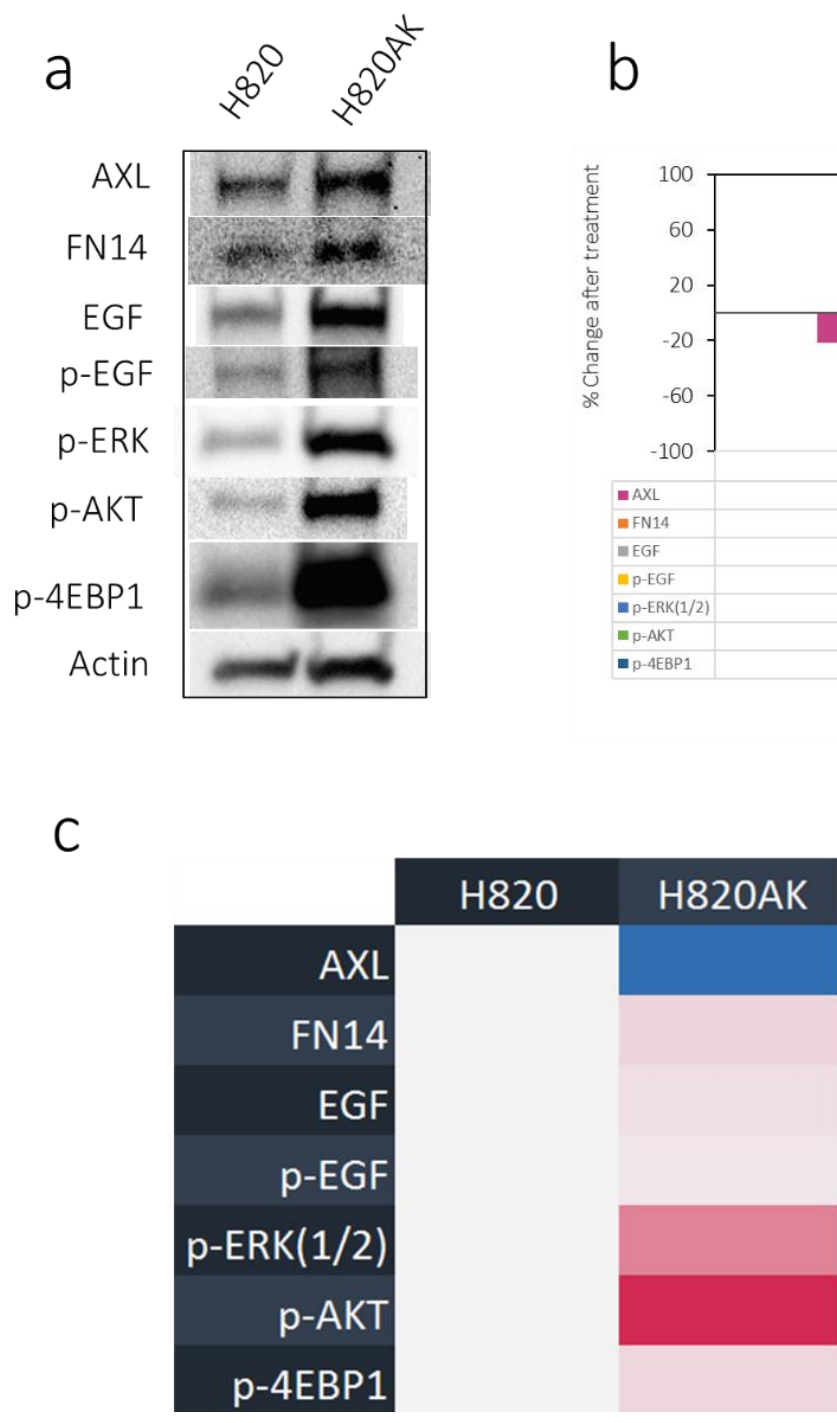

Figure 114. Protein analysis between H820 and CRISPR generated AXL knockout H820AK cells: (a) associated blot and (b) Band densitometry analysis for blot and (c) Representative heat map showing relative expressions (Red=high; Blue=low).

\subsection{4 mRNA and miRNA expression of different oncogenic factors towards AXL}

RT-PCR were performed to analyze the effect of AXL down regulation. Western blots results suggest that AXL protein was affected by both siRNA and crRNA. Although we see a change in protein levels required for pathways to function, several studies have documented presence of feedback loops to resist such changes. These feedback mechanisms are closely associated with mRNA expression in cells. Additionally, 
several studies have shown that AXL is also responsible for regulating proliferative pathways by upregulating the mTOR pathway ${ }^{227}$. Upregulation of mTOR is followed by downregulation of apoptosis signaling mechanism within cells 264,265 . This conversely meant AXL could theoretically restrict the P53 gene expression required for normal autophagy processes 217,266 . Moreover, studies have also confirmed links between non-coding DNAs (ncDNA) in regulating compensatory feedback loops ${ }^{267-269}$. The ncDNA and their counterpart miRNAs within the cells, can directly promote gene expression within the cell nucleus ${ }^{270-272}$. Therefore, we investigated the expression of certain mRNAs and miRNAs associated with AXL. For this study we first evaluated the difference in AXL and P53 mRNA levels within the cells both before and after AXL downregulation. Additionally, we also examined the effect of synergistic drug treatment. Similar to protein analysis, results from RT-PCR show that AXL gene expression is downregulated after treatment with siRNA or crRNA (Figure 115). Interestingly, P53 expression increased when AXL was downregulated by siRNA, suggesting that autophagy processes could be restored after siRNA treatment (Figure 115). Apoptosis assay results performed using annexin and flow cytometry showed an increase in late stage apoptosis in cell corroborating RT-PCR analysis (Figure 116). Still, P53 expression remain unchanged in CRISPR treated cells, that meant a compensatory activation could be restricting P53 expression. These activations may be controlled by other proliferation related oncogenes such as EGFR ${ }^{273,274}$. Furthermore, studies have shown that EGFR is linked with regulation of AXL through certain downstream pathways ${ }^{217,275}$. This linkage may be a part of a feedback loop to promote 

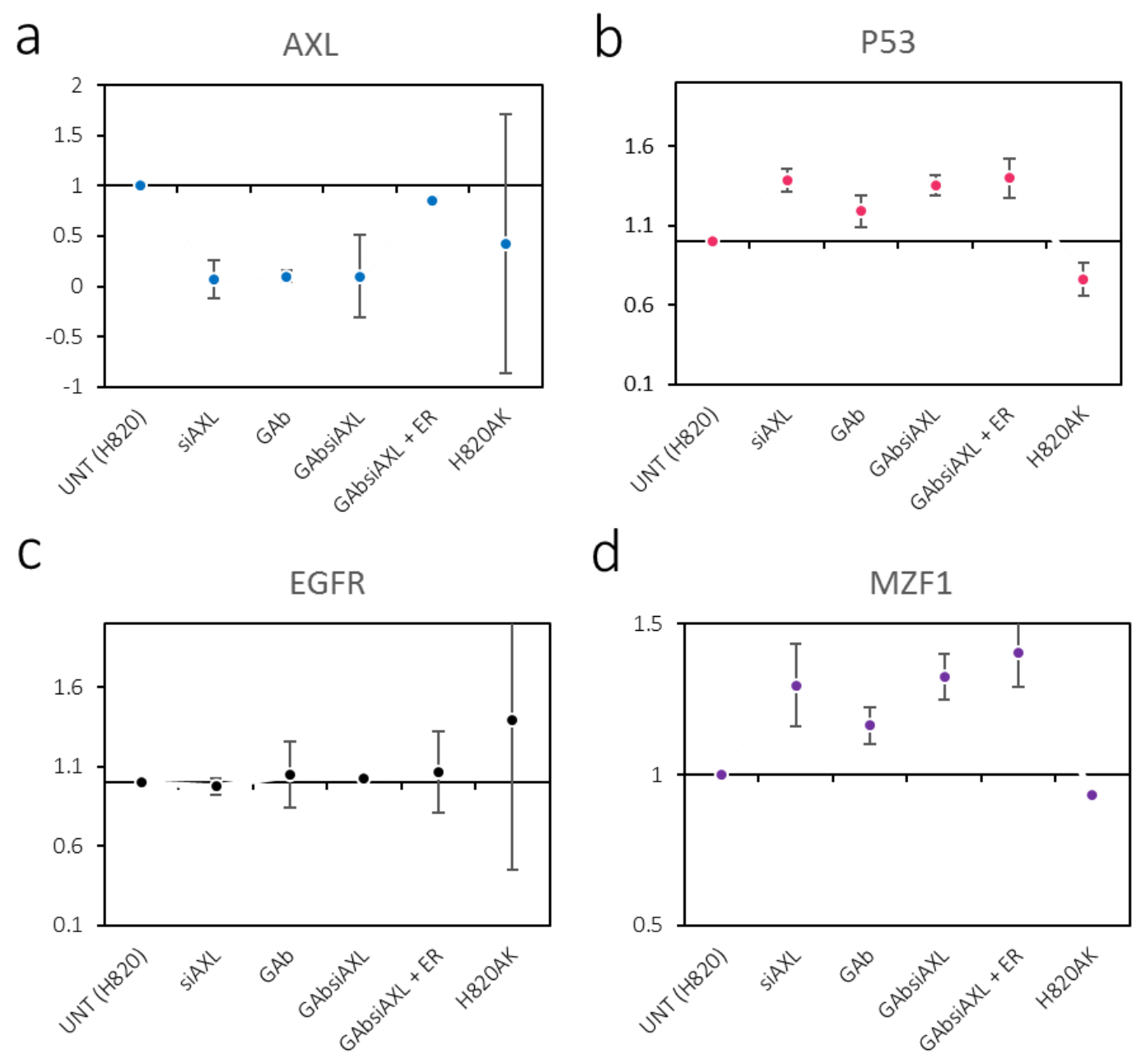

Figure 115. RT-PCR gene expression analysis for (a) AXL, (b) P53, (c) EGFR, and (d) MZF1. Results were averaged from 3 independent experiments.

AXL expression in the nucleus. Interestingly, an earlier work suggested the role of Myeloid zinc finger 1 (MZF1) in directly promoting the AXL gene within the nucleus $^{276}$. Additionally, MZF1 has been shown to be responsible for regulating proliferation and invasion that is closely related to drug resistance in cells ${ }^{276,277}$. Based on these studies, it is plausible for EGFR to upregulate when AXL is downregulated. It is also possible for MZF1 to upregulate in order to equilibrate AXL expression in cells. 
a

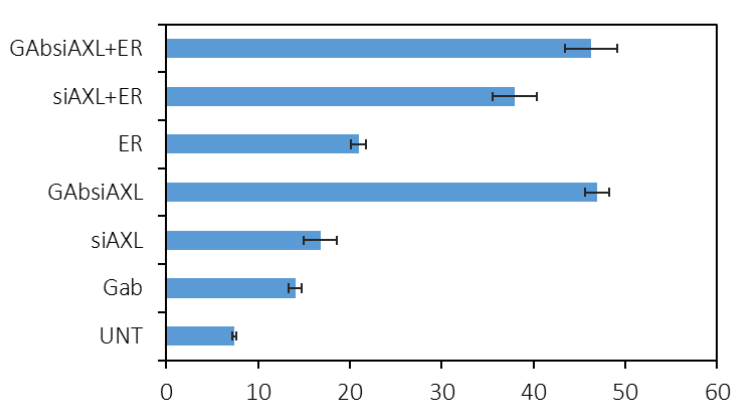

b
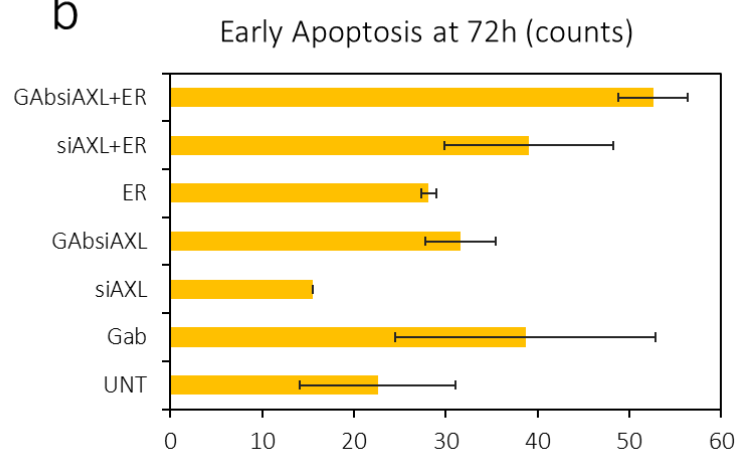

C

$48 \mathrm{~h}$
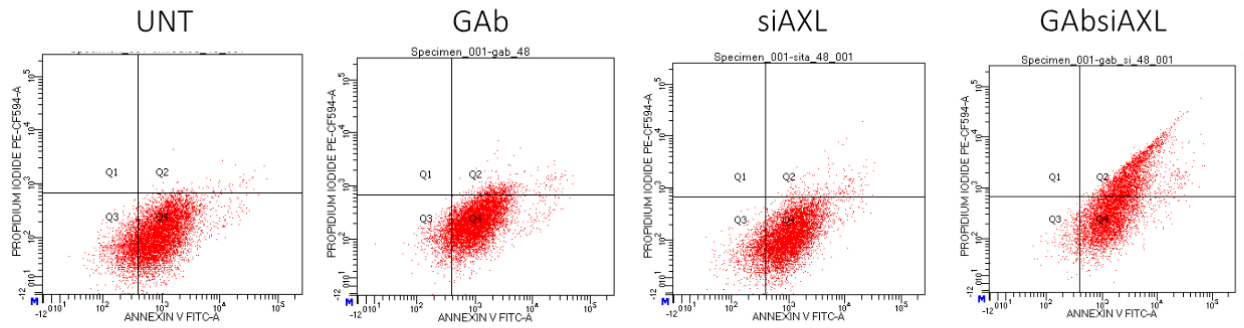

$72 \mathrm{~h}$
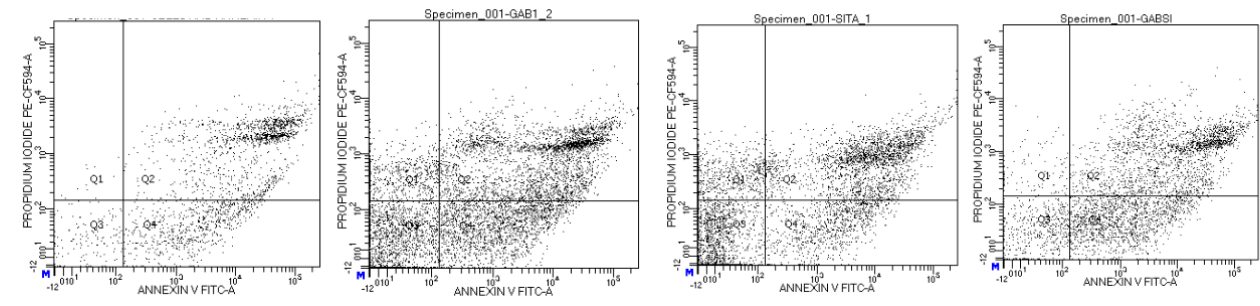

$48 \mathrm{~h}$
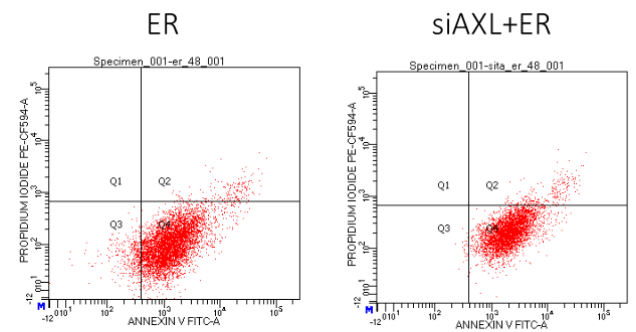

GAbsiAXL+ER
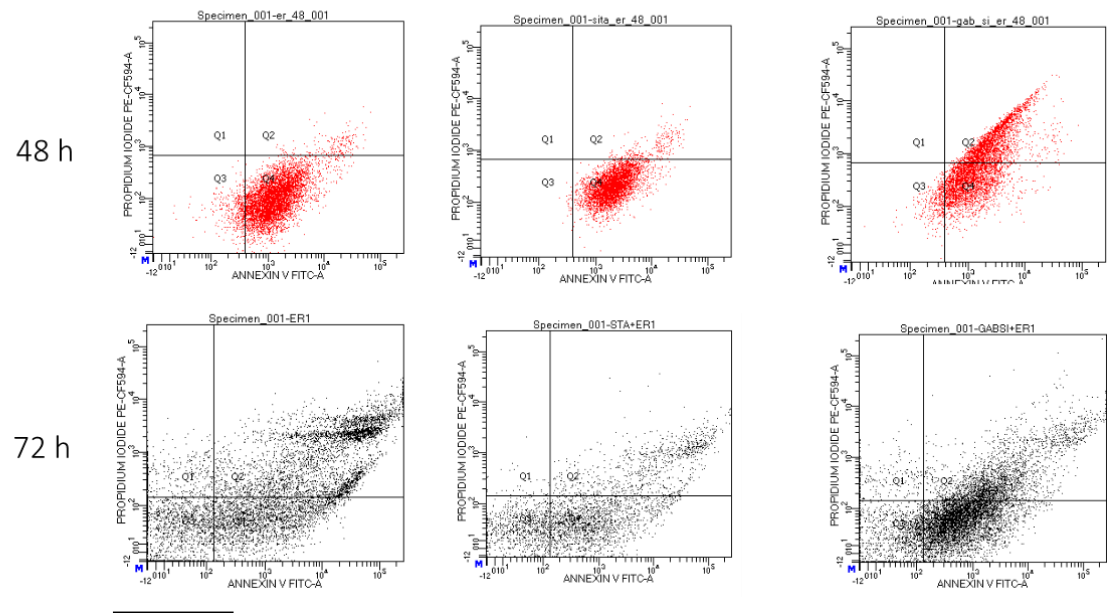

Figure 116. Apoptosis assay analysis for (a) Late-stage at $48 \mathrm{~h}$ and (b) Early-stage at $72 \mathrm{~h}$ shows relative increase during synergistic treatment of siRNA based samples with erlotinib in H820 cells. (c) Associative flow cytometry data for apoptosis results. Q2 and Q4 indicate late and early-stage apoptosis respectively. Results were averaged from 2 independent experiments. 
Therefore, we decided to investigate EGFR and MZF1 mRNA levels that have an association with AXL or drug resistance. However, the EGFR levels remained unchanged in siRNA treatments except for crRNA treatment (Figure 115). Results showed 1.3 fold increase for EGFR expression in H820AK cells. This data suggests a compensatory behavior through EGFR in cells. Conversely, a decreased expression was noticed for MZF1 in H820AK cells, wherein the gene was mutated and non-functional (Figure 115). Additionally, MZF-1 expression increased for synergistic drug treatments.
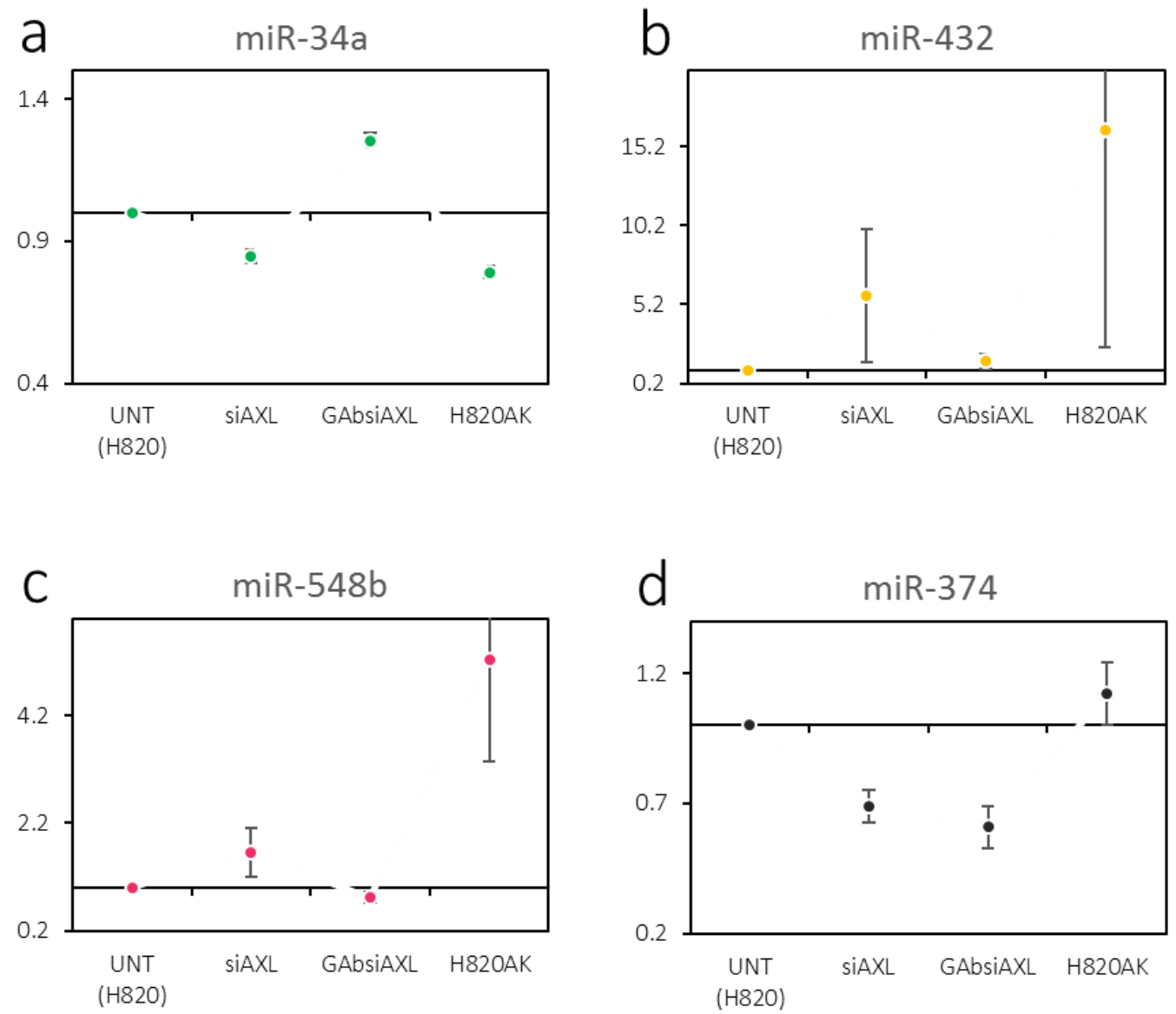

Figure 117. RT-PCR micro-RNA expression analysis for (a) miR-34a, (b) miR-432, (c) miR-548b, and (d) miR-374. Results were averaged from 3 independent experiments. 
This data suggests MZF1 association with AXL might be affected in CRISPR treated cells. Both these results suggest a compensatory behavior when the AXL gene is knocked down. As a next step we investigated the change in multiple miRNA associated with AXL. Firstly, we were interested in examining miR-34a that is controlled by P53 266, 278. Interestingly, the expression levels of miR-34a increased in cells when targeted by siRNA conjugated construct (Figure 117). This result suggests that antibody and siRNA had independent or mutual roles affecting and allowing miR34a to regain control. In a previous study, it was shown that miR-34a could directly deregulate AXL protein levels in cells ${ }^{266}$. The result signifies that miR34 regulation is related to P53 upregulation when AXL is targeted using antibody targeted construct, GAbsiAXL. However, the expression of miR34a did not increase in CRISPR treated cells correlating with P53 expression. Along with miR-34a, an earlier study on AXL upregulated cancer cells confirmed the link of two miRNAs; miR432 and miR548b that negatively regulate $\mathrm{EMT}^{279,280}$. Therefore, we hypothesized that these miRNAs might increase with AXL is downregulated. However, even though results showed that there was an increase in miR-432 and miR-548b for both siRNA and crRNA treated samples; the overall expression was minimal (Figure 117). Interestingly, a study showed that miR-548b is deregulated in drug resistant NSCLC and its expression is often targeted by miR-374 280 , 281. Therefore, we investigated miR-374 that has been shown to upregulate EMT in cancer cells. Indeed, RT-PCR results shows a decreased expression of miR-374 in siRNA and crRNA treated samples (Figure 117). Again, in CRISPR treated cells, the miR374 was slightly upregulated. These results suggest that, there are wider compensatory mechanisms at play and therefore several other proteins might be 
influencing these expressions. However, when the AXL mRNA is downregulated using siRNA, results suggest that the compensatory changes are relatively less. Therefore, in order to understand if these cells still had inherent proliferative potential after any compensatory activation, it is important to investigate downstream EMT regulation. To confirm this, we next investigated the invasion/migration related pathways that could be regulated by AXL.

\subsubsection{AXL regulates MMP-2 and MMP-9 in drug resistant NSCLC}

There are studies confirming that, drug resistant tumors with upregulated $\mathrm{AXL}^{82,215} 80$, 228 often have EMT characteristics that include invasive and migrative abilities ${ }^{224,237}$. Thus, it is possible that AXL directly regulates EMT expression. We performed invasion/migration assay to assess the EMT potential ${ }^{282,283}$. Results showed a down trend when AXL was targeted by siRNA or crRNA (Figure 118).

a

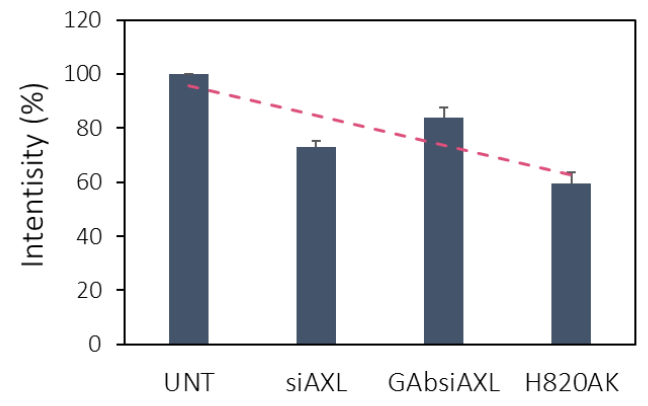

b

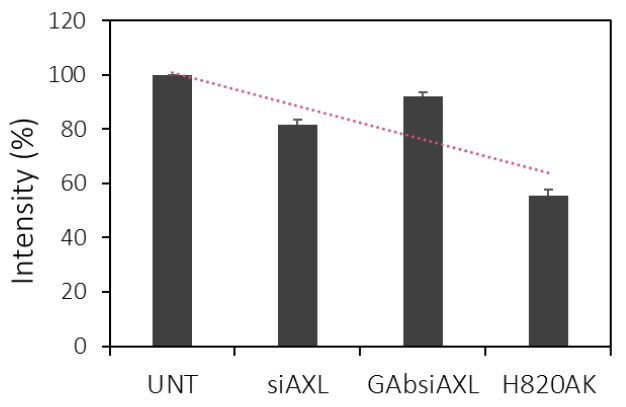

Figure 118. Proliferation analysis in H820 cells using (a) Invasion assay and (b) Migration assay.

However, the decrease in siRNA treatments was not significant. This can be due to the fact that these assays involve quantification of nucleic acids after lysing the cells. Since siRNA based treatments were performed in the upper chambers of the assay, there could 
be false positive signals. In order to remove this limitation, we wanted to focus on a more specific analysis. Therefore, we investigated MMP-2 and MMP-9 levels in the cells using zymography ${ }^{223,}{ }^{284}$. Several studies have shown that AXL expression was correlated with high MMP expression in cells ${ }^{285}$. MMP expression was directly related to invasiveness and migratory capacity in cells. In fact, MPP are highly expressed in cell undergoing EMT required for metastases ${ }^{77,286,287}$. Therefore, by investigating MMP expression in cells both before and after treatments with siRNA based constructs, we could accurately investigate the role of AXL. For this study, we investigated the MMP expression in synergistic treatments with drug, and expression in CRISPR treated H820AK cells. Strikingly, we noticed a decrease in both MMP-2 and MMP-9 levels in the cells (Figure 119). The results obtained were similar to those reported in literature, wherein they showed AXL could upregulate MMP-9223. Similarly, in another study AXL was shown to regulate MMP-2 in mucoepidermoid carcinoma ${ }^{288}$. Interestingly, we notice a sharp decrease of MMP-2 in H820AK cells with non-functional AXL gene (Figure 119). These results indicate AXL is directly responsible for regulating MMP-2 expression and partly regulating MMP-9 expression in drug resistant H820. For understanding how AXL affects the EMT pathway we also analyzed associated proteins such as Vimentin, N-cadherin and E-cadherin post AXL knockdown. For this whole study we had 2 treatment groups and three controls. The 2 groups included (1) siRNA and siRNA based construct treatment and (2) synergistic treatment of siRNA and siRNA based constructs along with drug. For the controls we had untreated cells and drug treated cells and Gab without siRNA. 

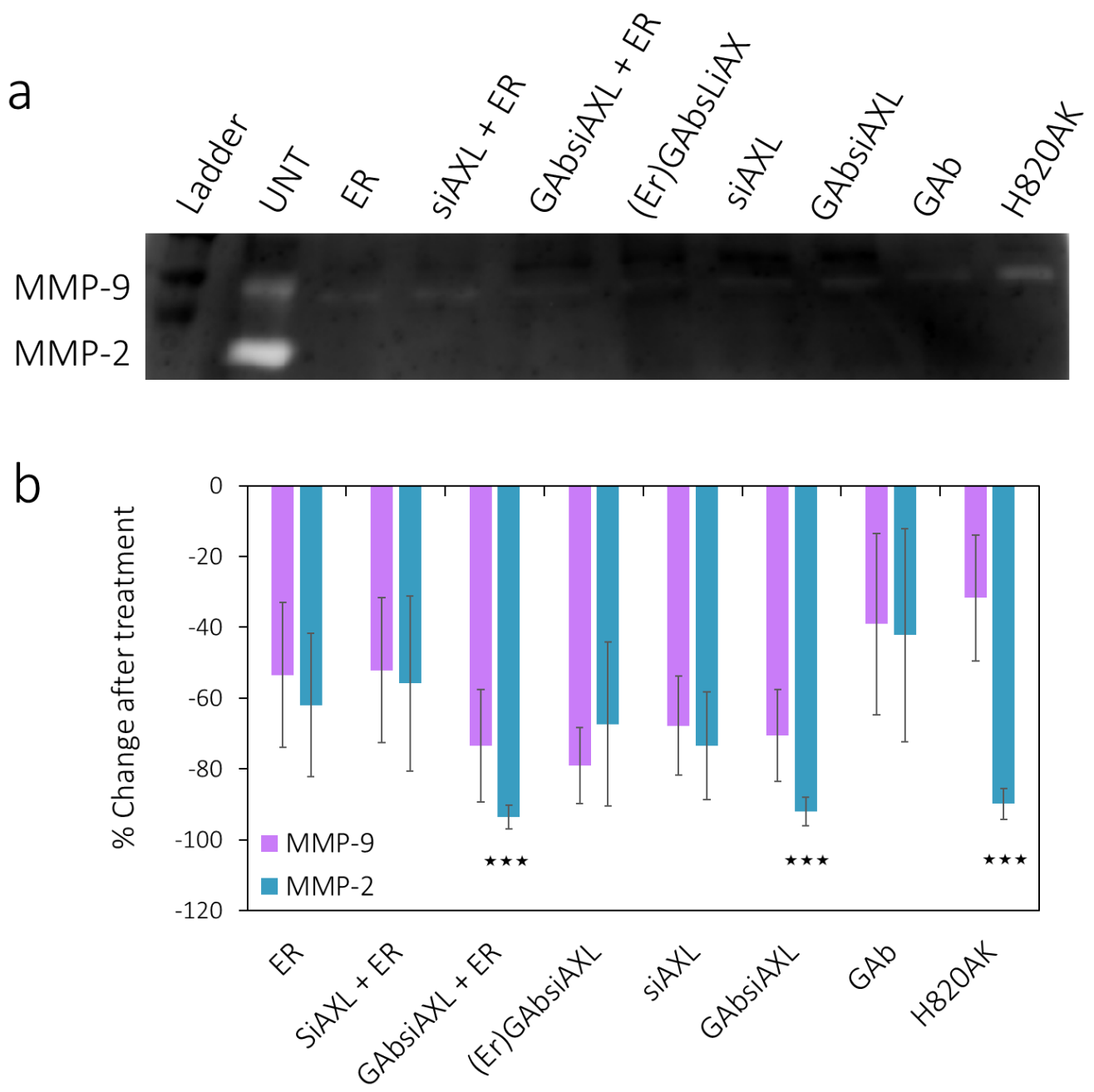

Figure 119. Zymogram analysis in H820 cells for MMP-2 and MMP-9 activity using conditioned media: (a) Associated zymograph (b) Band densitometry analysis. Results were averaged from 3 independent experiments. ${ }^{\star \star \star} \mathrm{p} \leq 0.001$, and the values were analyzed using one-way anova.

For analysis of protein we compared all samples to untreated control. Remarkably, protein analysis showed a reduction in Vimentin and $\mathrm{N}$-cadherin expression corroborating zymogram data (Figure 120, 121 and 122). Based on earlier studies, the results confirm that EMT pathway was downregulated ${ }^{76}$. Thus by regulating EMT and MMP levels, AXL might be increasing the proliferative abilities of the cell. 
These abilities require high energy consumption and protein synthesis ${ }^{289}$. Several studies have shown that AXL regulated mTOR and mTOR pathway which increases protein synthesis $^{227}$. Consequently, we investigated several proteins downstream of mTOR pathway after targeting AXL.

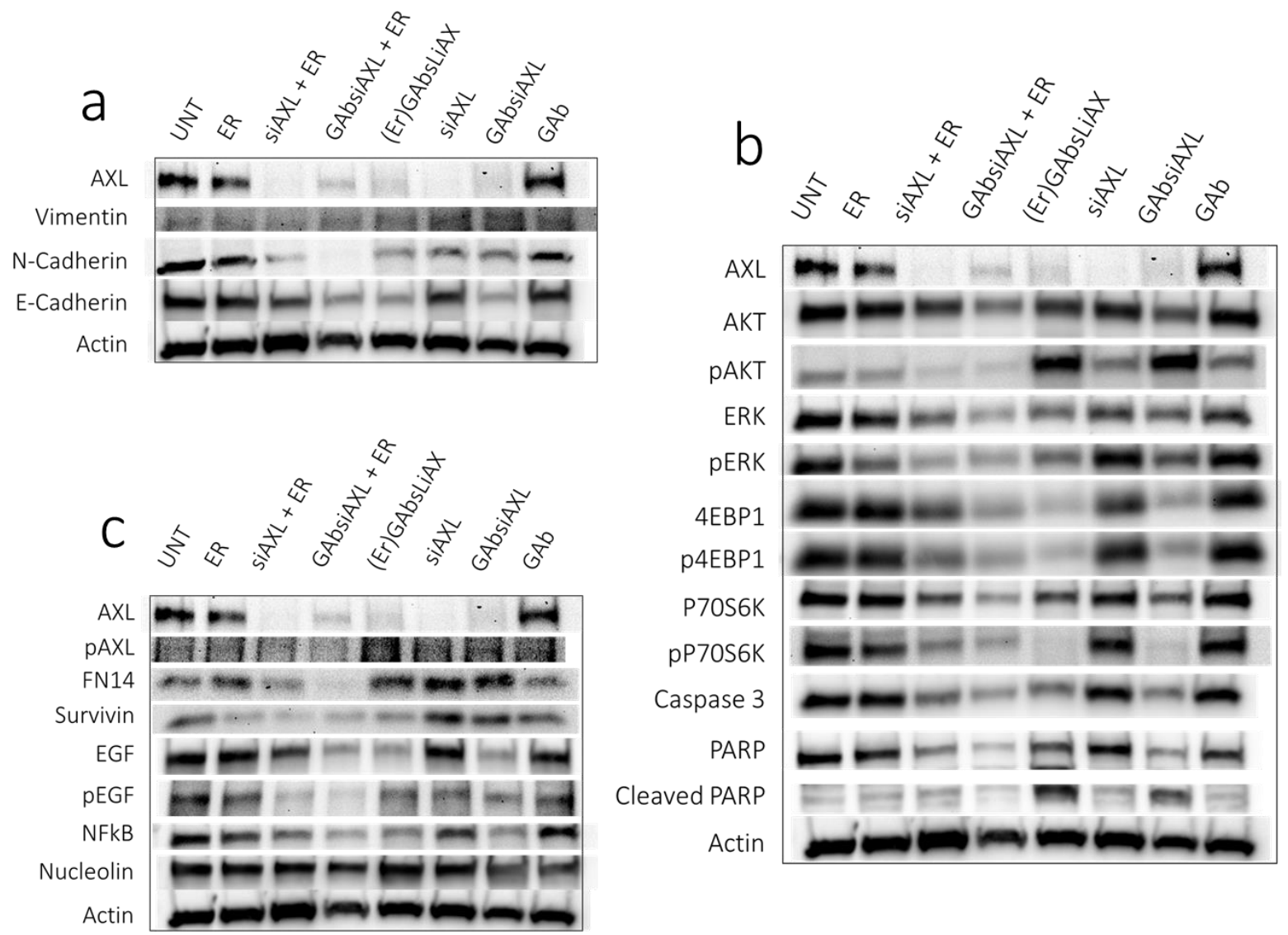

Figure 120. Western blot for AXL siRNA based treatments, controls and synergistic treatment with erlotinib in H820 cells: (a) EMT pathway (b) mTOR-AKT axis and apoptosis pathway and (c) EGFR and associated survival pathways. 


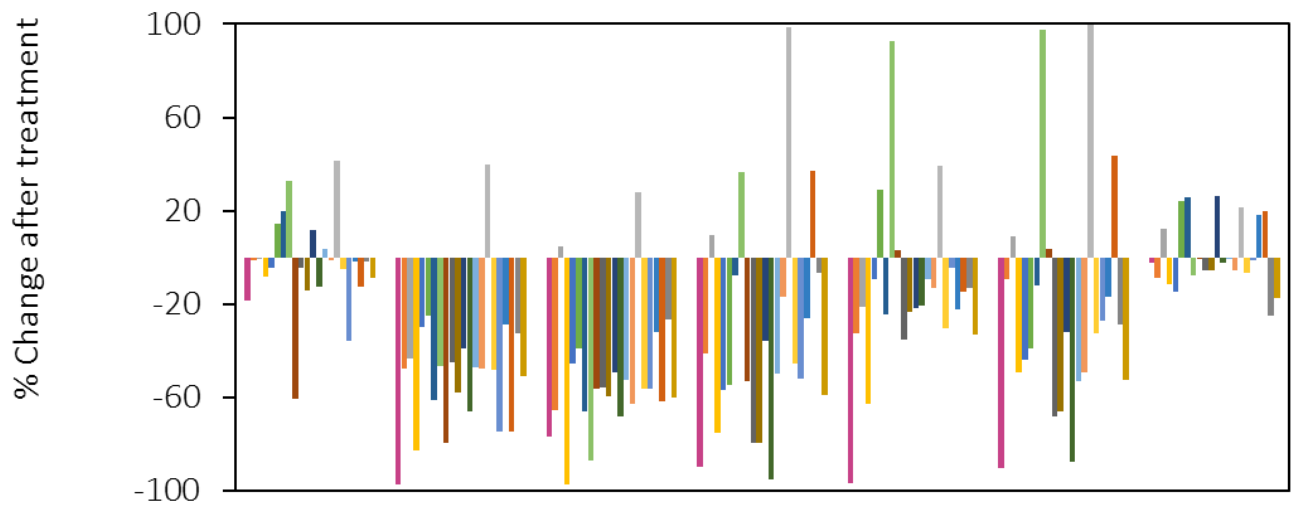

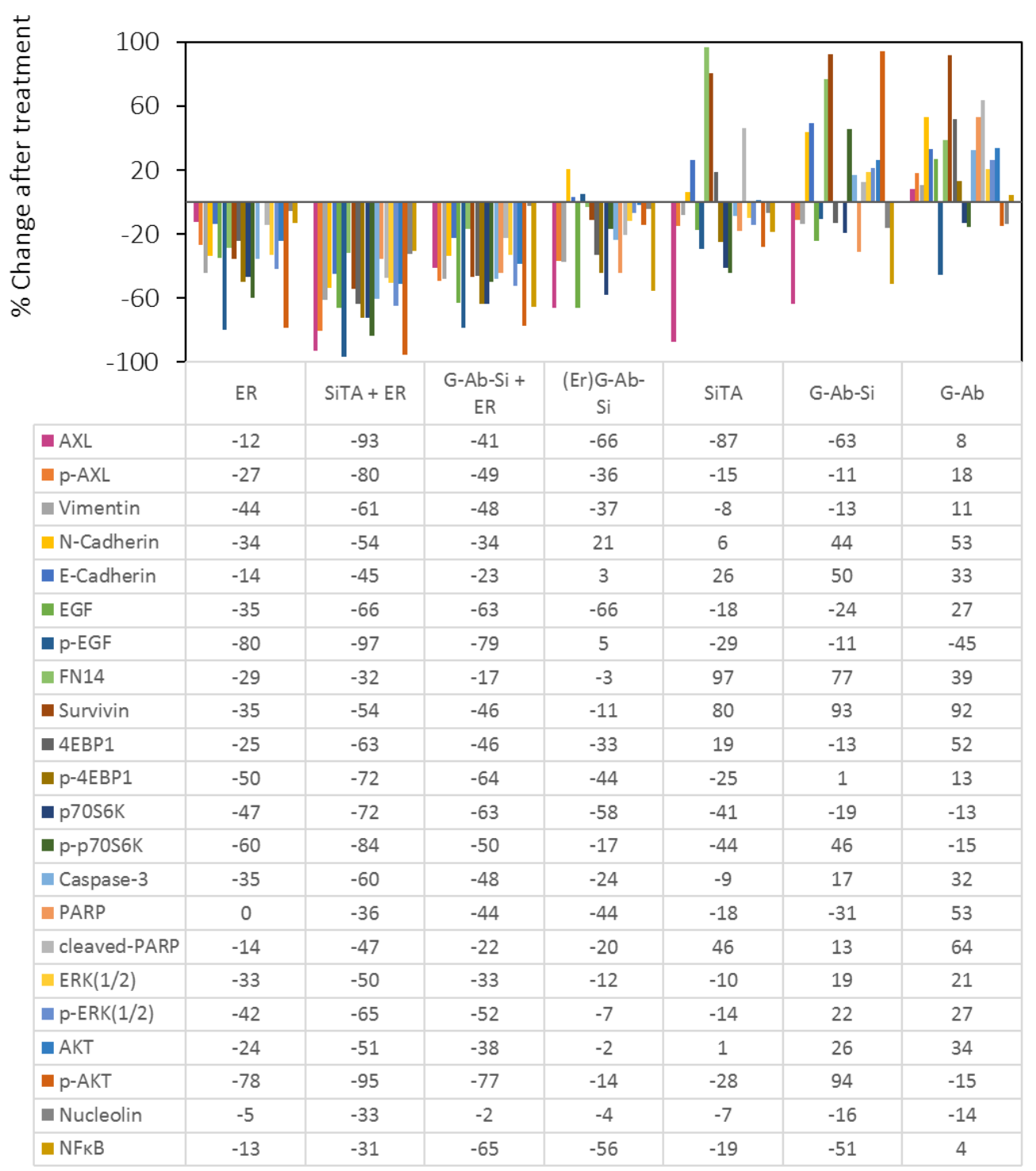

Figure 121. Band densitometry analysis for associated blots shown in Figure 120. Data represents protein analysis for AXL siRNA based treatments, controls and synergistic treatment with erlotinib in H820 cells. 


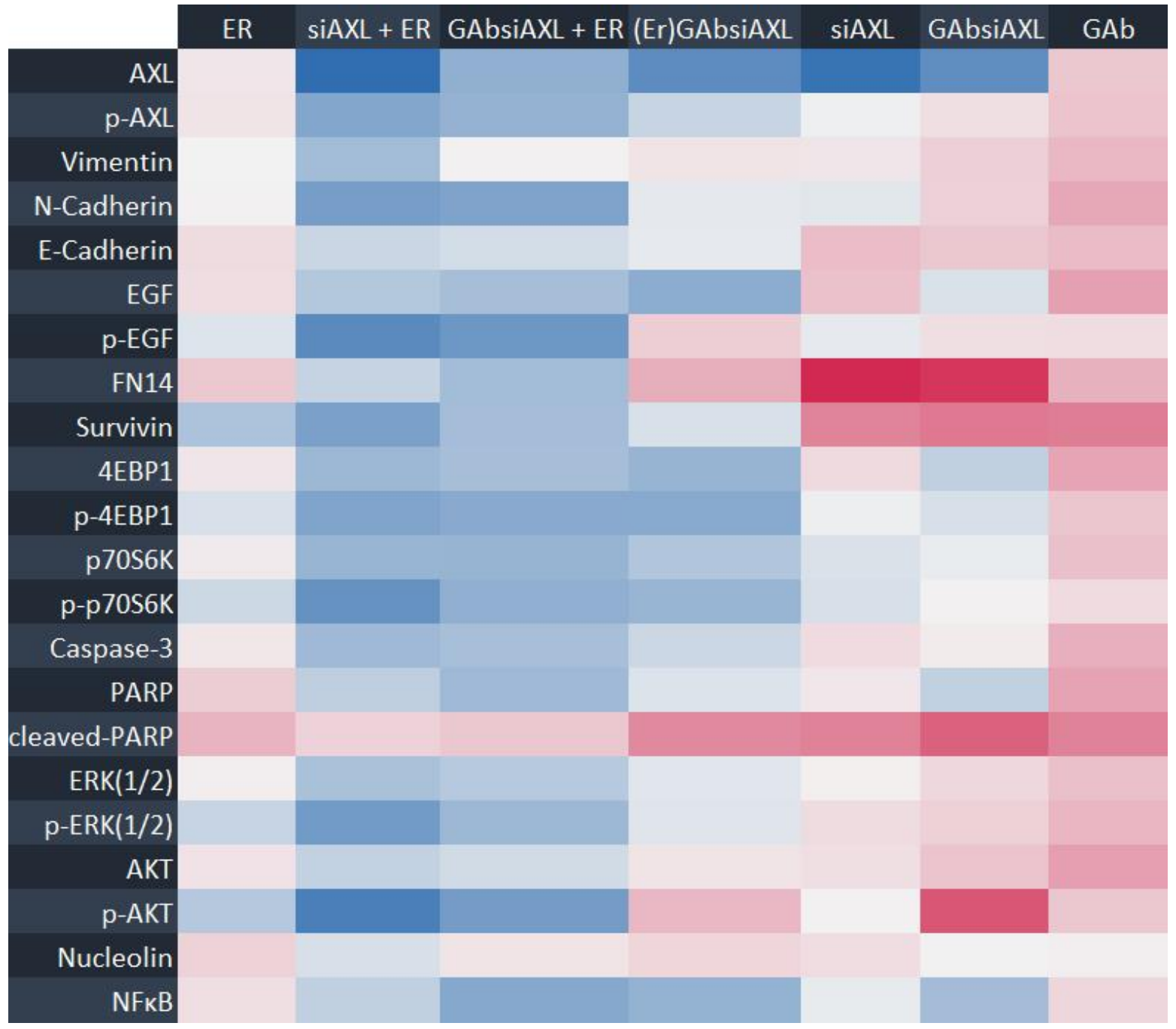

Figure 122. Representative heat map for data shown in Figure 121 (Red=high; Blue=low). Data represents protein analysis for AXL siRNA based treatments, controls and synergistic treatment with erlotinib in H820 cells. Results were averaged from 2 independent experiments.

\subsubsection{AXL knockdown deregulates mTOR pathway in NSCLC}

AXL has been shown to upregulate the AKT pathway downstream ${ }^{221}$. In turn AKT pathway activates the mTOR pathway ${ }^{226}$. The mTOR complexes that get activated are known to phosphorylate 2 major proteins, 4EBP1 and p70S6K ${ }^{290,}{ }^{291}$. This phosphorylation results directs protein synthesis, cell growth and energy production by 
glycolysis. This effect is also known to be part of the Warburg hypothesis in cancer cells, wherein the normal mitochondrial energy cycle is arrested ${ }^{292}$. In normal cells, 4EBP1 binds to eIF4F complex that controls and recruit's ribosomal proteins ${ }^{290}$. However, in cancer cells, phosphorylation of 4EBP1 inactivates this process. On the other hand, phosphorylation of p70S6K mediates mitogenic signals by arresting the $\mathrm{G}$ to $\mathrm{S}$ phase checkpoint for normal cell cycle regulation ${ }^{291}$. Both these processes contribute to proliferation. In order to investigate the effect of AXL on mTOR, we therefore studied 4EBP1, p70S6K and their phosphorylated counterparts. Furthermore, the activation of mTOR complexes is known to arrest the P53 pathway that controls normal cell growth factors and cell death substrates ${ }^{265}$. In normal cells these substrates, caspase-3 and poly(ADP-ribose)polymerase (PARP) are activated for execution of apoptosis $^{293}$. Typically, it leads to cleavage of PARP that initiates apoptosis. However, during mTOR upregulation, these cell death and repair mechanism are suspended and therefore growth is unchecked ${ }^{293}$. So, we also investigated these proteins that are required for the normal cell death processes. We treated H820 cells with same treatment groups used for EMT analysis. Results from erlotinib treated cells showed minimal effect (Figure 120, 121 and 122). However synergistic siRNA treatment with drug, downregulated most of the mTOR associated downstream proteins (Figure 120, 121 and 122). siRNA treated cells showed an upregulation for normal 4EBP1 and a decrease in phosphorylated p70S6K. On the other hand, GAb showed an upregulation in most proteins (Figure 120, 121 and 122). Similarly, siRNA based constructs with antibody did not decrease mTOR associated proteins. This signifies antibody treatment might also have a synergistic effect. Interestingly, even CRISPR treated AXL knockout cells 
showed relatively higher amounts of phospho-4EBP1 (Figure 114). However, the cleaved PARP levels required for normal apoptosis function increased in siRNA and siRNA based constructs (Figure 120, 121 and 122). These results indicate that AXL downregulation affects the mTOR pathway and upregulates normal apoptosis functions in drug resistant NSCLC.

Next, we investigated upstream activators of mTOR that are ERK and AKT pathways $^{226,294}$. Both EGFR and AXL are known to activate these pathway ${ }^{84}$. Results show that synergistic treatment of EGFR inhibitor along with AXL siRNA downregulated both these pathways as compared to drug control (Figure 120, 121 and 122). On the other hand, AXL knockout cells, H820AK showed an upregulation of both phospho-ERK and phospho-AKT (Figure 114). The analysis from H820AK show that a new pathway upstream of AKT and ERK had been activated. Additionally, we also investigated downstream proteins often associated with nuclear gene promoters such as nucleolin and $\mathrm{NFkB}^{223,295,296}$. Results showed that NFkB a downstream target of AKT, was downregulated in all drug-siRNA based treatments (Figure 120, 121 and 122). These results suggest that NFkB was being controlled by AXL and downregulation of both EGFR and AXL had a combined effect. Similarly, siRNA and antibody based construct also showed a downregulation (Figure 120, 121 and 122). However, when treated independently with erlotinib or GAb NFkB was upregulated (Figure 120, 121 and 122). Surprisingly, siRNA treatment alone had minimal change (Figure 120, 121 and 122). Together these results signify that a pathway downstream of EGFR was initiating a pro-survival signal. This signal was strong enough that, the siRNA treatment alone had minimal change of NFkB levels (Figure 120, 121 and 122). When we 
examined EGFR levels, most samples showed minimal change except synergistic treatments (Figure 120, 121 and 122). Furthermore, even CRISPR generated H820AK cells showed upregulated EGF protein (Figure 114). Therefore, based on these results we ascertained that AXL survival cross-talk was in play and the pathway in question was related to EGFR.

In order to investigate this possibility, we looked at oncogenic survival factors namely survivin and FN14 that are associated with EGFR ${ }^{297,298}$. Survivin was an inhibitor of apoptosis while FN14 was a wound healing gene ${ }^{290,299}$. Recently, FN14 was shown to be upregulated in cancer and had a link to cachexia ${ }^{300}$. Cachexia associated with muscle loss due to protein degradation, worsens disease progression in cancer patients during drug treatment ${ }^{300}$. Additionally, FN14 was also implicated in drug resistance in cancer ${ }^{301-303}$. A bioinformatics analysis also suggested a link between AXL and FN14 pathways downstream ${ }^{304}$. Remarkably, our protein analysis showed that both survivin and FN14 where upregulated when AXL was inhibited (Figure 120, 121 and 122). Additionally, upregulation of FN14 was consistent with increased NFkB expression (Figure 120, 121 and 122). Previous studies also confirmed that NFkB is a downstream target of FN14 ${ }^{305-307}$. To confirm the data, we repeated the results for FN14 analysis, and results showed a consistent upregulation of $\sim 80 \%$ every time AXL was downregulated (Figure 123). This led us to believe FN14 that is closely related to EGFR, was part of a AXL survival cross-talk. This discovery led us to question whether this cross-talk was present only in H820 cells and was cell line dependent. 


\section{AXL inhibition}

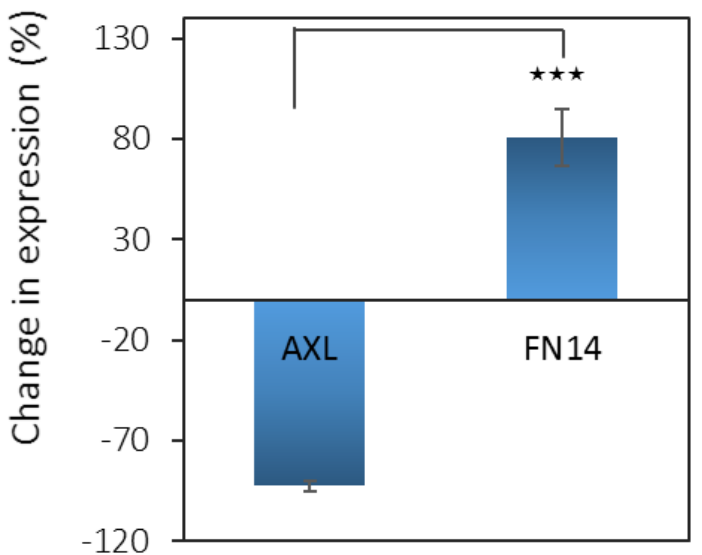

Figure 123. Relative expression of FN14 when AXL is downregulated in H820 cells. Results were averaged from 3 independent experiments. ${ }^{\star \star \star} \mathrm{p} \leq 0.001$, and the values were analyzed using one-way anova.

\subsubsection{AXL crosstalk with FN14 may be independent of EGFR mutant status}

The AXL-FN14 cross-talk was investigated in several other cells. FN14 was indeed found to be upregulated along with AKT, ERK, EGF and downstream mTOR protein in H820AK gene knockout cells (Figure 114). The downregulation of AXL in KRAS mutant A549 NSCLC cells resulted in upregulation of FN14 (Figure 124). AXL was also downregulated in another EGFR T790 mutant similar to H820, H1975 that was known to have upregulated FN14. In H1975, FN14 upregulation was not significant (Figure 124). These results signify that the AXL-FN14 cross-talk axis is EGFR mutant independent. Also, if FN14 was already upregulated in H1975 cells, FN14 might be part of a cross-talk involving other pathways rather than $\mathrm{AXL}^{297,301}$. Overall, these results suggested that downregulation of AXL led to FN14 upregulation in order for cells to survive. This survival strategy by FN14 leads to protein degradation in drug resistant NSCLC, and was EGFR mutant independent. 

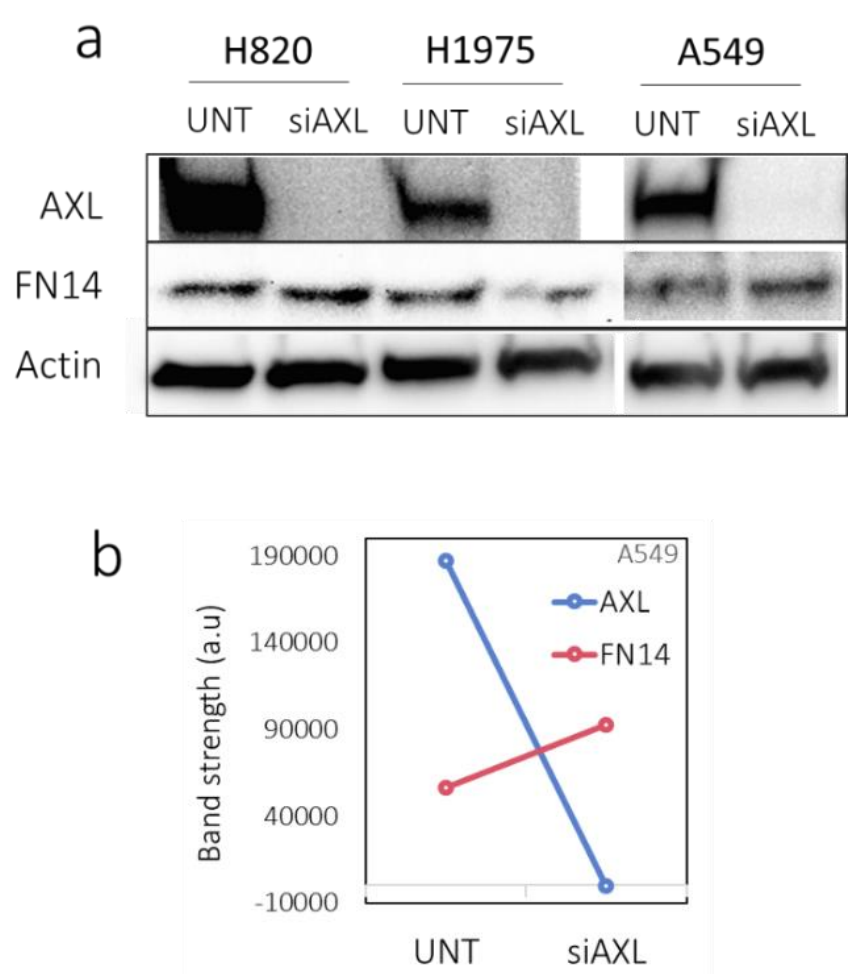

Figure 124. Relative expression of FN14 when AXL is downregulated in NSCLC cells: (a) associated western blot and (b) Band densitometry analysis for A549 cells showing FN14 upregulation.

\subsubsection{Mechanism of AXL-FN14 and its role in TKI resistance}

To understand how FN14 upregulates we investigated proteins that may be regulated both by AXL and FN14 independently. Most importantly, a previous study had found FN14 to be downstream of TGF $\beta$ pathway ${ }^{308}$. In this study, TGF $\beta$ via SMAD4 was shown to increase FN14 gene expression. SMAD proteins often translocate to the nucleus to start transcription of various genes ${ }^{309}$. Thus, SMAD proteins are often controlled by oncogenes to regulate growth related genes. Indeed, several studies have shown AXL via JNK pathway could control SMAD3 proteins as well ${ }^{310}$. It is known that SMAD3 and SMAD4 can form complexes to translocate into the nucleus ${ }^{311}$. 
Furthermore, in AXL upregulated cells, it was shown that AXL deregulates TGF $\beta$ in an earlier study ${ }^{310}$. This step ceases TGF $\beta$ induced apoptosis regime. Together the results from these studies suggest, TGF $\beta$ might upregulate in absence of AXL and could directly regulate FN14 to kick-start a survival mechanism. To investigate this hypothesis, we looked at several of these proteins and other nuclear transcriptor proteins implied in this process. Specifically, we looked at 4EBP1 that is associated with mTOR, and FoxO family proteins. Similar to SMAD, FoxO proteins have a fork head DNA binding domain that can directly regulate cell growth genes ${ }^{289}$. Generally, in cancer cells, these proteins are phosphorylated by the AKT pathway and taken out of the nucleus ${ }^{312}$. Therefore, if these proteins are deregulated it would signify that AKT or ERK pathway may have been re-initiated in AXL downregulated cells. Indeed, protein analysis revealed an upregulation of both TGF $\beta$ and SMAD proteins (Figure 125 and 126). Interestingly, Adam17 a sheddase protein that is part of a positive feedback loop controlled by FN14 increased it expression as well (Figure 125 and $126)^{313}$. Results also indicated an increase in phospho-Jun that is part of the SAPK/JNK pathway (Figure 125 and 126). Importantly, when FN14 was upregulated, a slight increase in both AKT and ERK proteins are seen. These data indicate increased activity in SAPK/JNK, AKT and ERK pathways that might be regulated by EGFR. Results also showed a decrease in phospho-4EBP1 and phospho-p70S6K, and an increase in Caspase3, indicating an increase in normal cell cycle control when AXL pathway is inhibited. This data was similar to the previous protein analysis (Figure 120, 121 and 122). On the other hand, when we downregulated both AXL and FN14 using siRNA 


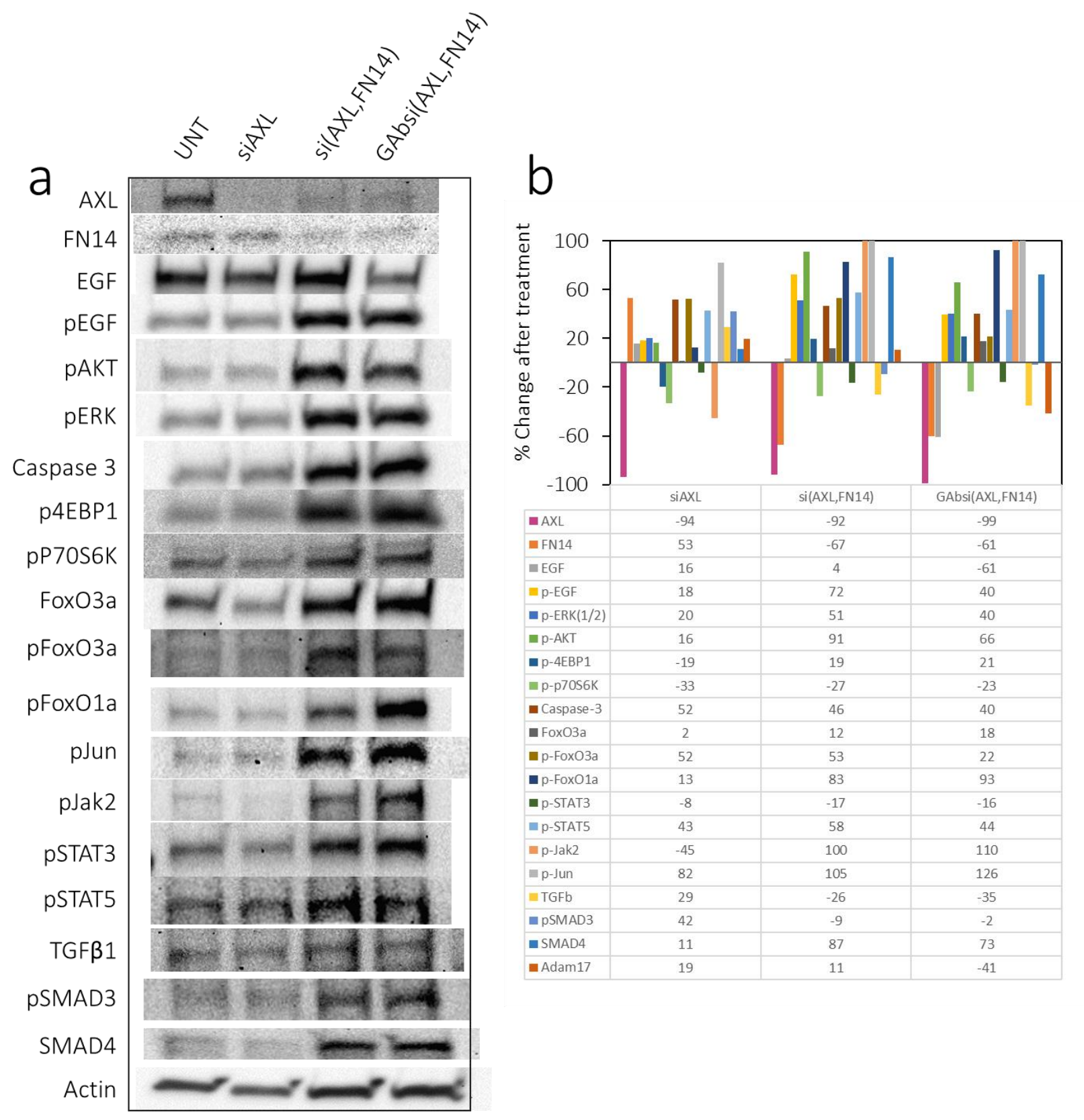

Figure 125. (a) Western blot for AXL-FN14 survival cross-talk analysis in H820 cells using siRNA and dual siRNAconstruct, GAbsi(AXL,FN14) treatments (b) associated band densitometry analysis.

and siRNA conjugated constructs, we noticed increased ERK and AKT activity, and phosphorylation of 4EBP1 and FoxO family proteins (Figure 125 and 126). We also noticed an uptick in JAK/STAT proteins that communicates with FN14, 


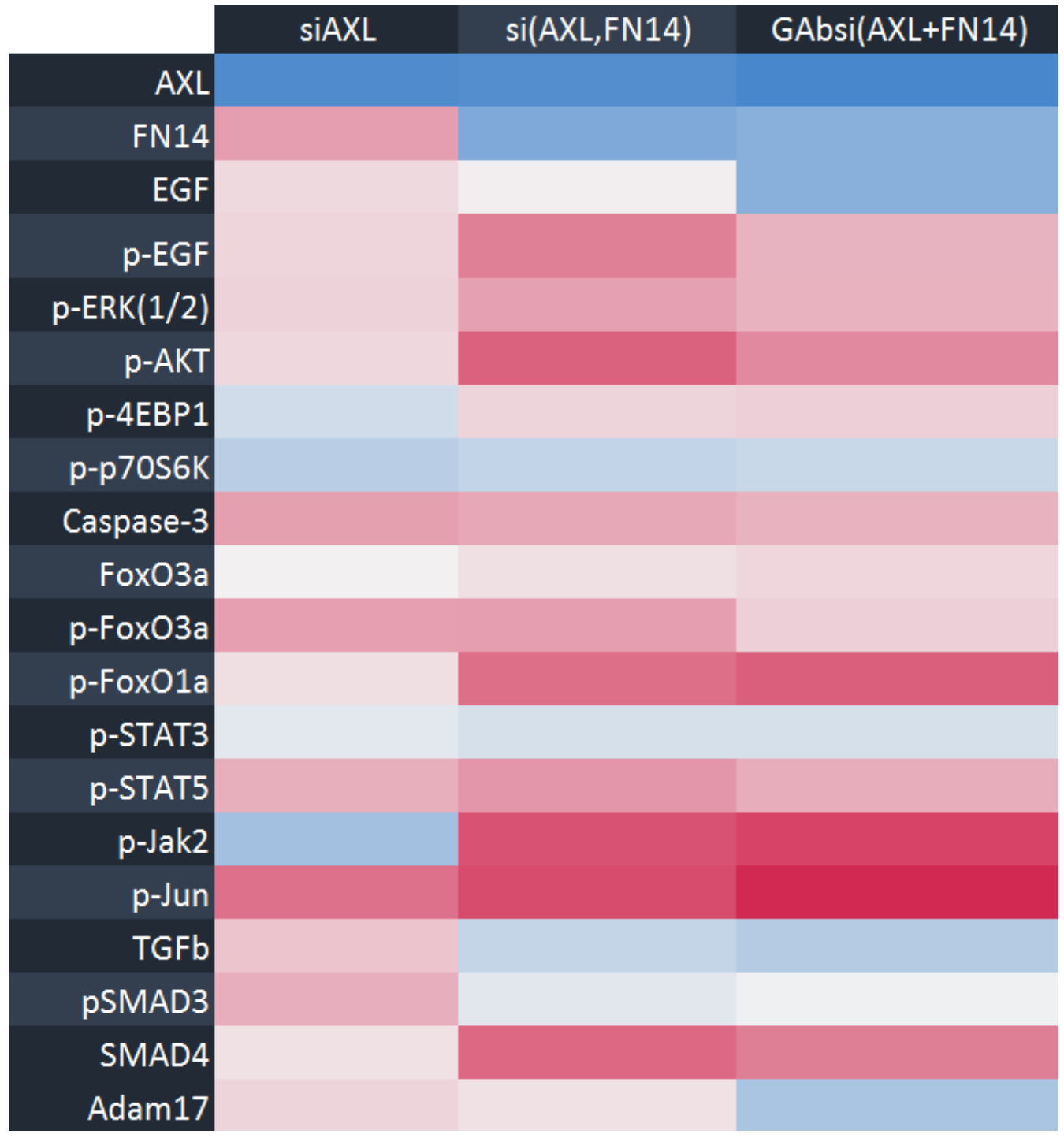

Figure 126. Representative heat map for data shown in Figure 125 (Red=high; Blue=low). Data represents protein analysis for AXL and FN14 siRNA based treatments in H820 cells.

and phospho-EGF (Figure 125 and 126). These results suggest EGFR may be compensating for the loss of both AXL and FN14. Compared to siRNA treatment, antibody conjugated constructs had minimal difference (Figure 125 and 126). These results suggest that EGFR blocking might be reducing the effect of phosphorylation in the cells. Alternatively, these results also suggest that other pathways may be involved in compensatory feedback loops. Overall, we hypothesize TGF $\beta$ may be 


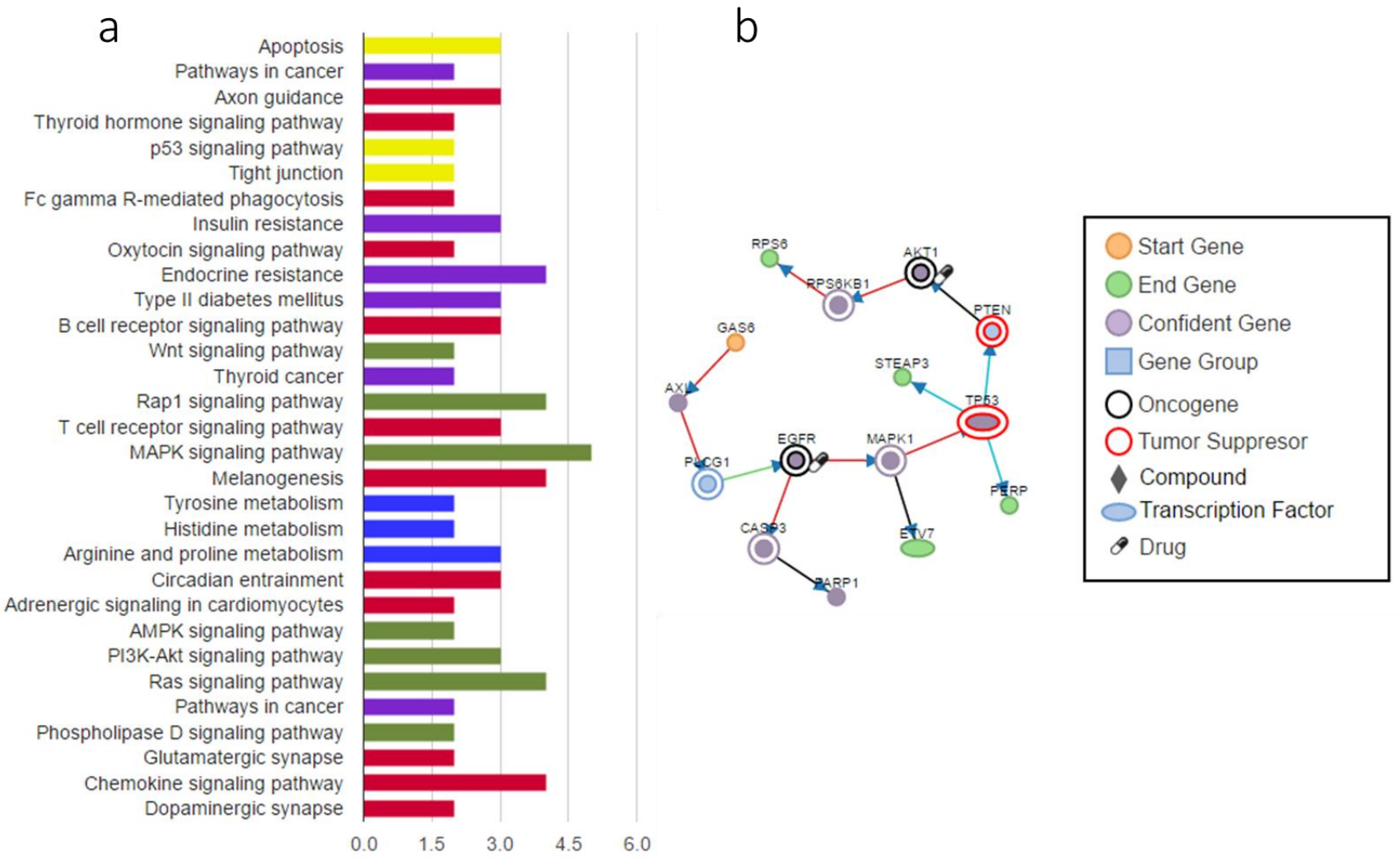

Figure 127. AXL and FN14 weighed gene network analysis: (a) Biochemical pathway association and (b) Downstream network association.

actively increasing FN14 expression through the SMAD family proteins when AXL is downregulated. However, in the scope of this study we are increasing the amount of data for specific protein cross-talks, that can help us to understand these mechanisms. Further analysis of other proteins may be needed to sufficiently authenticate all crosstalk signaling mechanisms. Therefore, for looking at associative relations between our data with other genes and miRNAs, bioinformatics analysis was also utilized. For this study, associations with several genes and miRNAs were analyzed using the upregulation/downregulation factors based on AXL and FN14. Using gene modelling, we first analyzed associative biochemical pathways and 


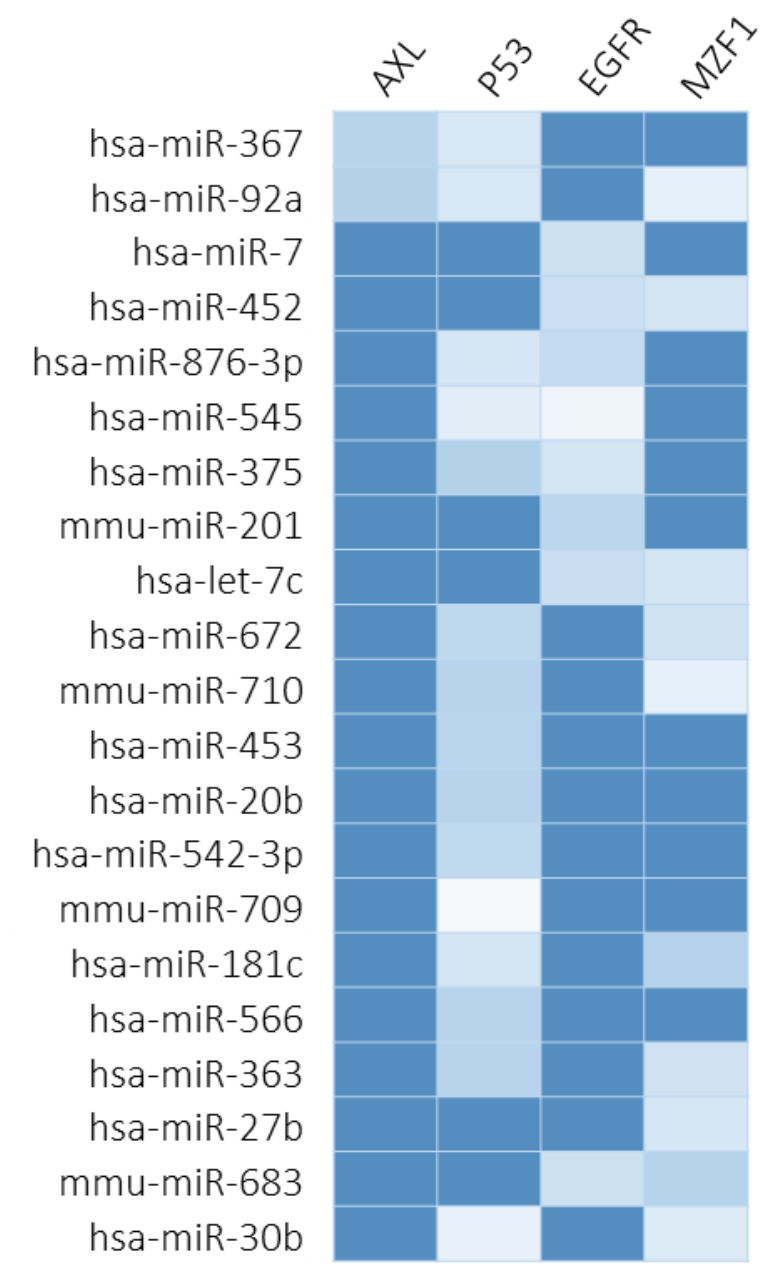

Figure 128. Micro-RNA association heat map based on experimental PCR data in H820 cells. (White=high; Blue=low). Data represents several miRNAs that scored for high associations with AXL, P53, EGFR and MZF1 out of 153 screened miRNAs.

metabolic factors. Results show that AXL and FN14 axis has high association with Rap1, MAPK, Ras and chemokine signaling pathways (Figure 127). Moreover, FN14 belongs to a chemokine pathway ${ }^{299}$. Additionally, gene network analysis showed that AXL may have a downstream effect on PTEN (Figure 127). Interestingly, a prior study experimentally observed that PTEN was upregulated when AXL was downregulated ${ }^{314}$. Furthermore, bioinformatic analysis of RT-PCR gene expression data revealed associations with several micro-RNAs (Figure 128). One of miRNAs that had high 


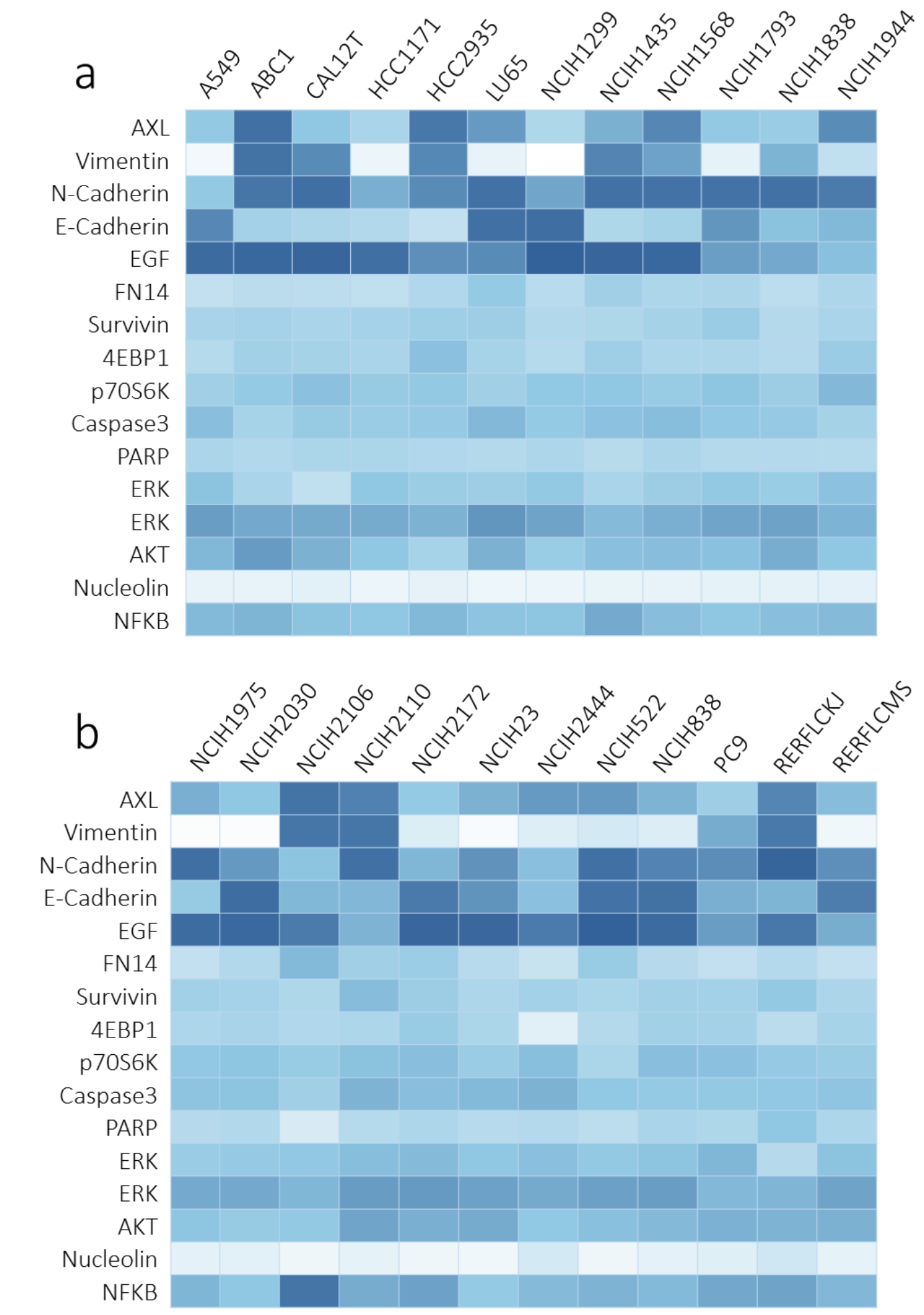

Figure 129. KEGG gene association heat map based on experimental PCR and protein data for lung cancer cells. (White=high; Blue=low). Data shows A549 and many other cells lines may high associative scores for AXL and FN14. 
scores in the analysis was miR-92a (Figure 128). miR-92a showed high associations with AXL, P53 as well as MZF1. Based on our experimental data and bioinformatic analysis, it is possible to suggest that miR-92 could be a target for AXL induced resistance in cancer cells. Interestingly, a recent study showed evidence of miR-92a in directly deregulating AXL and TGF $\beta^{315}$. Interpretation of these results signify the need to investigative several of these association to fully understand the role of AXL in NSCLC drug resistance. The results also confirm that AXL may have an important role in inducing and regulating several of these genes and miRNAs to impart resistance in NSCLC cells. In order to investigate the possibility of AXL-FN14 associations in lung cancer, we analyzed gene associations in several lung cancer cell lines. Based on the protein analysis, NSCLC cell lines showed high associations with both AXL and FN14 (Figure 129). In fact, AXL associations had higher scores than EGFR in several of these cell lines. Based on these data, we hypothesize AXL-FN14 compensatory axis may exist in multiple cell lines. Therefore, it is important to validate this cross-talk in various NSCLC cell lines by inhibiting AXL-FN14 cross-talk, and confirming loss of resistance to TKIs.

Based on this hypothesis we combined drug treatment with pathway inhibition and studied the effect of drug resensitization in cells. First we optimized the required concentration of DMSO and transfection agent needed for drug and siRNA treatments by testing their toxicity in H820 cells (Figure 130). Additionally, cells were checked for sensitivity to anti-EGFR antibody (Cetuximab). Results showed that T790M mutant H820 cells were more resistant to antibody than HCC827 cells (Figure 130). Next we established that siRNA alone had no effect on multiple cell lines (Figure 130). 
a

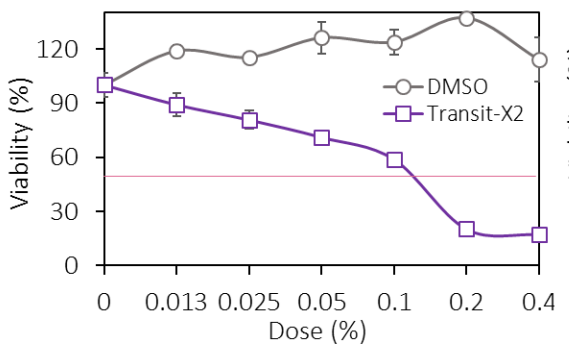

Dose (\%) b

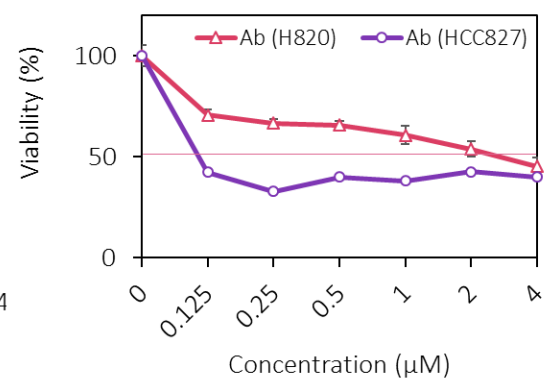

C $\leadsto$ siAXL $\leadsto$ siFN14 $\leadsto$ si(AXL, FN14) $\leadsto$ siMock

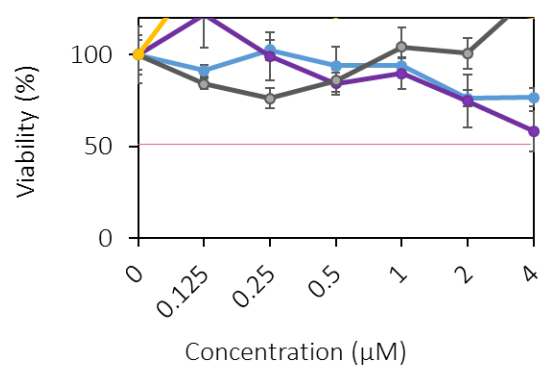

d

e

$\longrightarrow$ siAXL $\quad \longrightarrow$ siFN14 $\quad-$ si(AXL,FN14)

$\leadsto$ siAXL $\multimap$ siFN14 $\multimap$ siKRAS $\leadsto$ si(AXL,FN14)
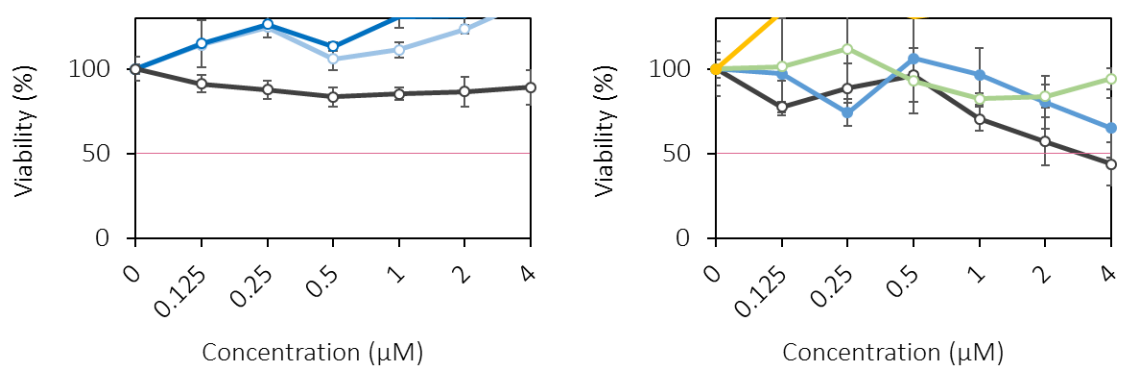

Concentration ( $\mu \mathrm{M})$

Figure 130. 72 h Cell viability analysis of (a) DMSO and Transit-X2 in H820, (b) Cetuximab antibody in H820 and HCC827 (c) siRNA in H820, (d) siRNA in H1975, and (e) siRNA in A549 cells.

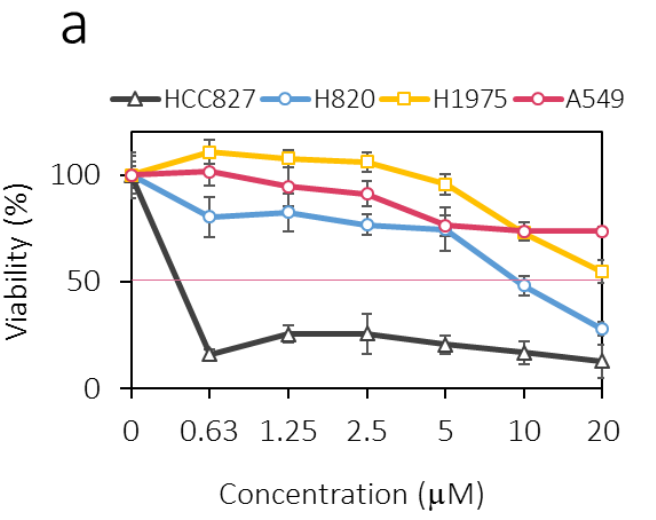

b

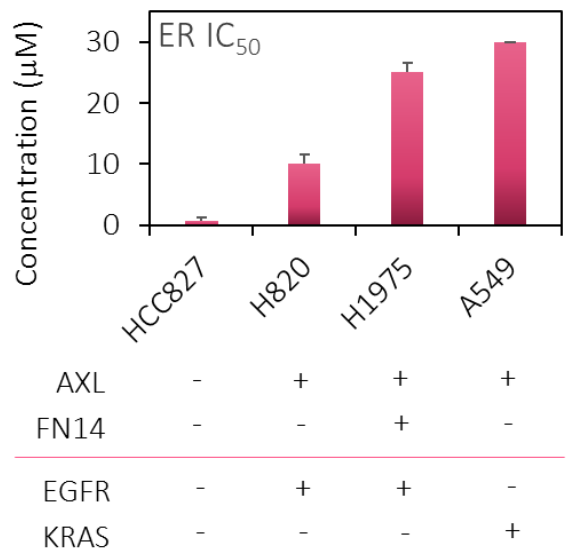

Figure 131. 72 h Toxicity of erlotinib in various NSCLC cells lines: (a) Cell viability plot and (b) IC 50 analysis. Results were averaged from 3 independent experiments. 

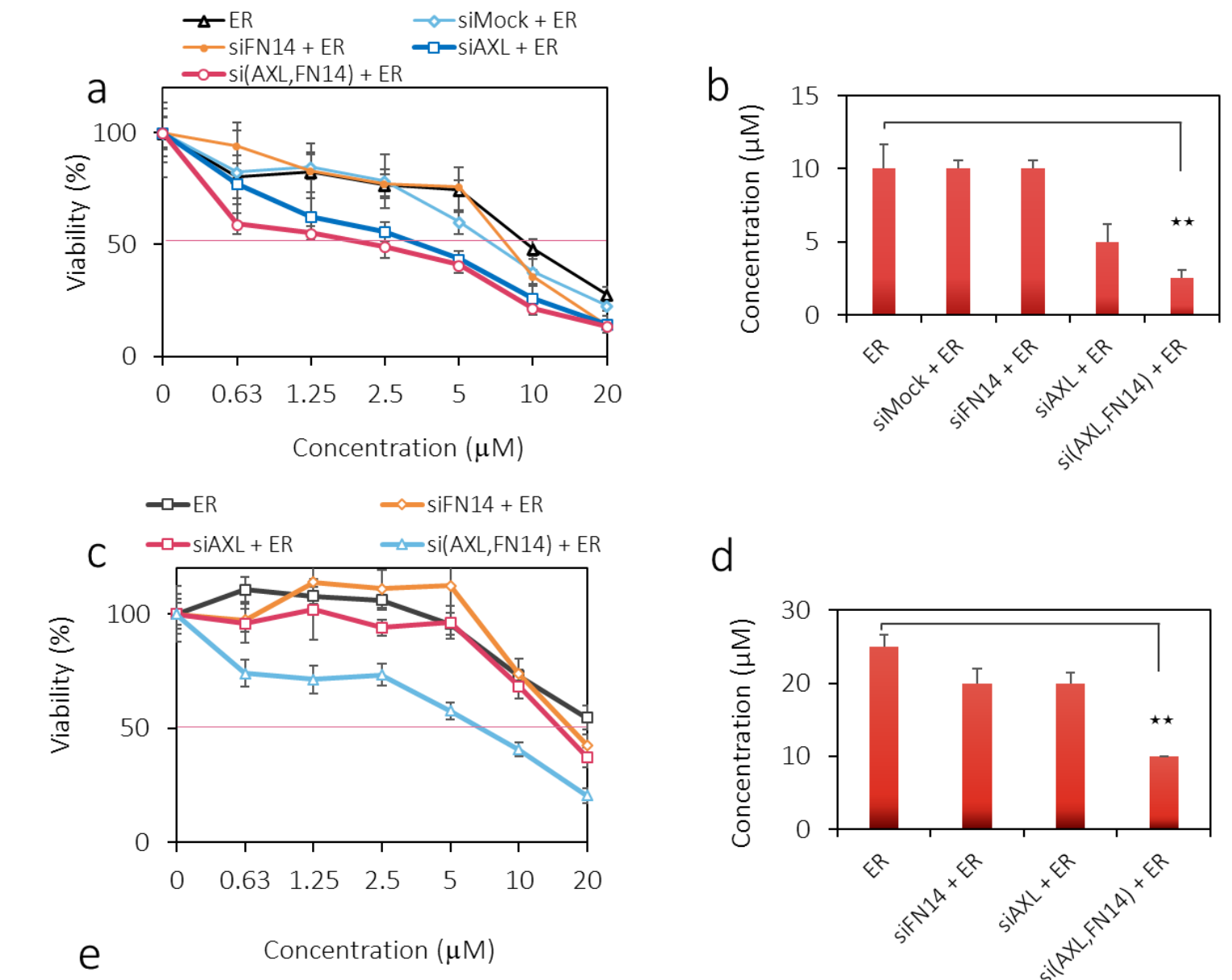

d
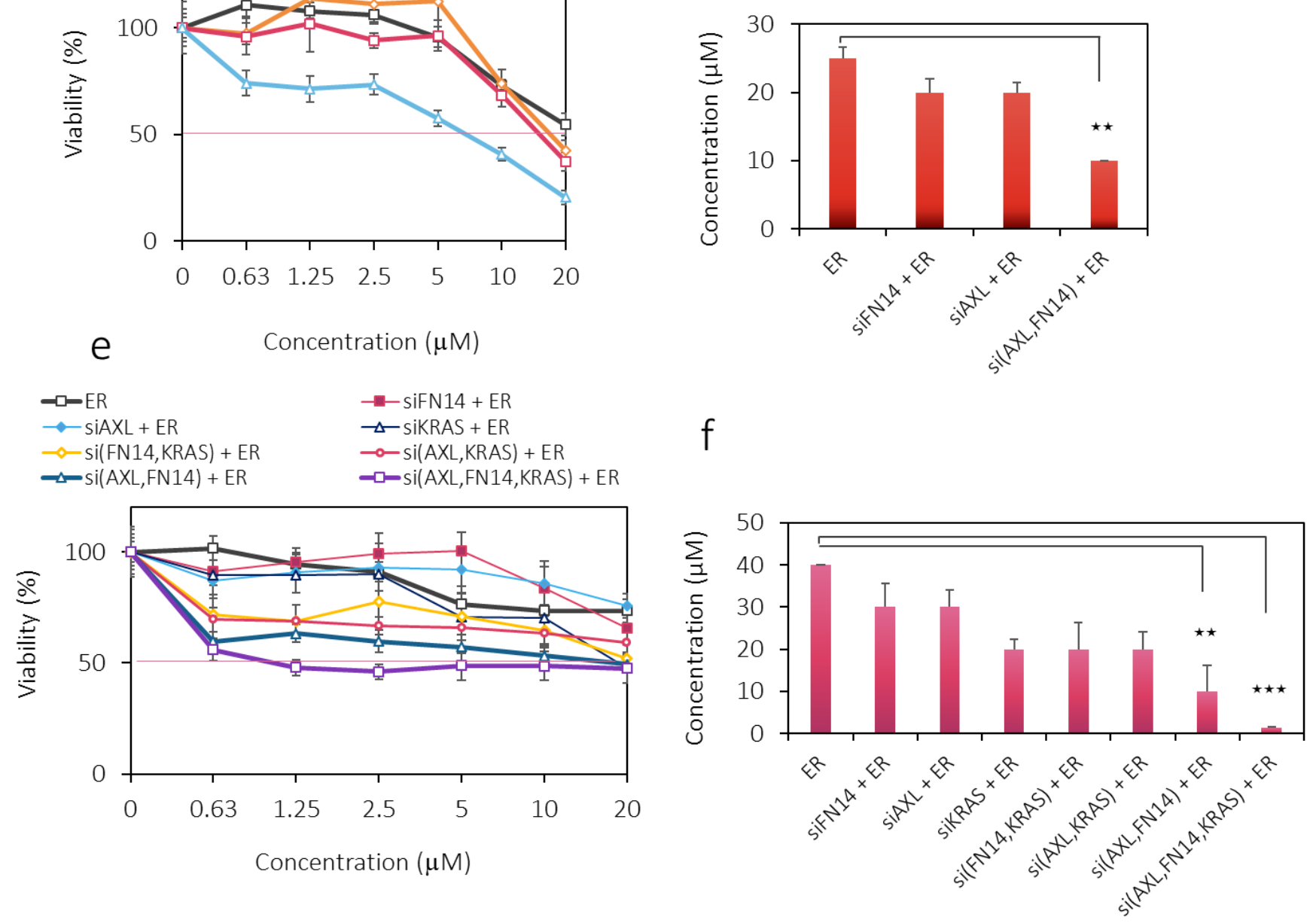

Figure 132. Resensitization to erlotinib in various NSCLC cells lines by blocking AXL/FN14/KRAS pathways in H820, H1975 and A549 NSCLC cells for 72 h. (a, c, e) Represent the cell viability plot and (b, d, f) represent the associated IC 50 analysis. Results were averaged from 3 independent experiments. ${ }^{\star} \mathrm{p} \leq 0.05,{ }^{\star \star} \mathrm{p} \leq 0.01,{ }^{\star \star \star} \mathrm{p} \leq 0.001$, and the values were analyzed using one-way anova. 
Additionally, the $\mathrm{IC}_{50}$ of erlotinib was investigated in multiple cell lines based on their AXL and FN14 expressions and EGFR/KRAS mutant status (Figure 131). Results showed that HCC827 that carries the E746-A750 deletion were highly sensitive while H820 that has both the drug sensitive del E746-E749 and resistant T790M mutation was resistant to erlotinib $\left(\mathrm{IC}_{50}>10 \mu \mathrm{M}\right)$. Similarly, the T790M mutant H1975 and KRAS mutant A549 were highly resistant to cells $\left(\mathrm{IC}_{50}>10 \mu \mathrm{M}\right)$. We also examined the independent and combined effects of AXL and FN14 siRNAs. We used both siRNA and siRNA based constructs for treatment and studied variation in $\mathrm{IC}_{50}$ of erlotinib in multiples drug resistant cell lines. Interestingly, downregulation of AXL resensitized the $\mathrm{H} 820$ cells to erlotinib by decreasing $\mathrm{IC}_{50}$ by half (Figure 132). Similar results were published in another study ${ }^{285}$. In A549, downregulation of AXL or FN14 was able to slightly decrease $\mathrm{IC}_{50}$ (Figure 132). We believed the KRAS mutation might be still allowing active downstream signaling in these cells ${ }^{257}$. Therefore, we also downregulated KRAS using a G12S mutation specific siRNA and determined that, $\mathrm{IC}_{50}$ for erlotinib had further decreased (Figure 132). However, when we knocked off both AXL (protein synthesis for proliferation) and FN14 (protein degradation for survival), the $\mathrm{IC}_{50}$ for erlotinib decreased in all three cell lines by half or more (Figure 132). In H1975, that already had FN14 upregulated, a combination of AXL and FN14 seemed to resensitize the cells (Figure 132). In addition, by downregulating all three pathways in A549, we could observe total resensitization of cells towards the drug (Figure 132). Together, these results suggest AXL is playing a role in erlotinib resistance in NSCLC. These results also signify AXL-FN14 axis can induce survival mechanism in drug resistant cells delaying action of drug. Still, these results are grounded only on the basis 
of the proteins examined during this investigation; a more comprehensive study including other cross-talk regulators is warranted.

\subsubsection{Targeting AXL-FN14 using nanoparticles in vitro}

Based on results from siRNA+drug toxicity studies, we investigated downregulate AXL-FN14 axis in H820 cells by treating with siRNA conjugated constructs. For this study we initially tested toxicity of gelatin nanoparticles in H820 and HCC827 cells. Results showed that particles after glutaraldehyde quenching were highly non-toxic (Figure 133). The studies also confirmed the ability of gelatin nanoparticles to deliver drug through encapsulation in HCC827 drug sensitive cells (Figure 133).

a

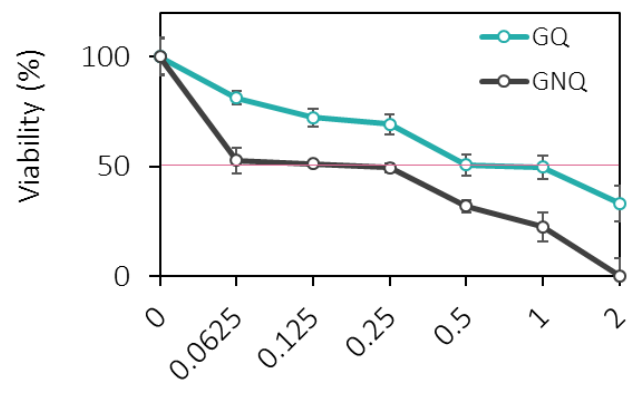

C Concentration $(\mathrm{mg} / \mathrm{ml})$

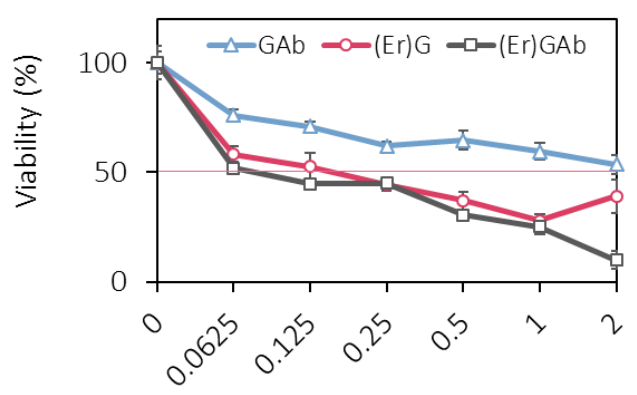

Concentration $(\mathrm{mg} / \mathrm{ml})$ $b$

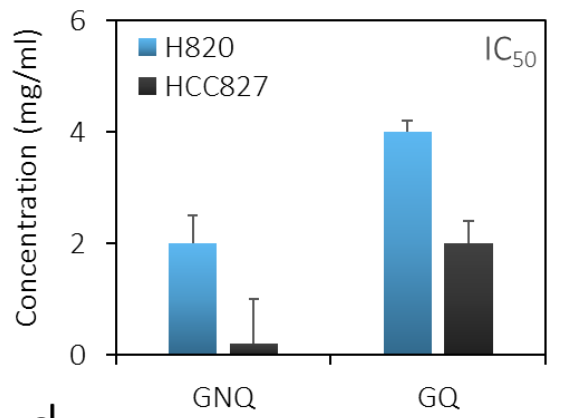

d

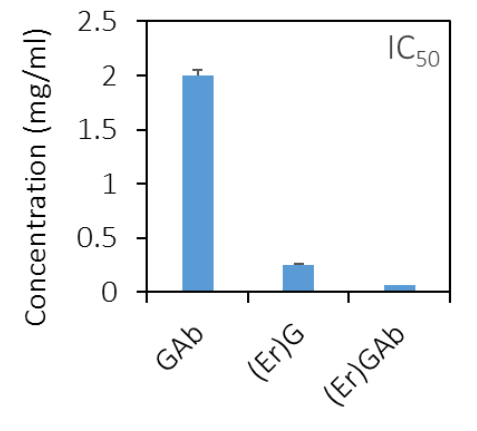

Figure 133. $72 \mathrm{~h}$ Toxicity profile for nanoparticles in H820, and HCC827 NSCLC cells. (a) Synthesized gelatin quenched and non-quenched NP and its representative (b) IC 50 analysis, (c) Comparison between drug encapsulated nanoparticles, and its representative (d) IC 50 analysis. Results were averaged from 3 independent experiments. 
a

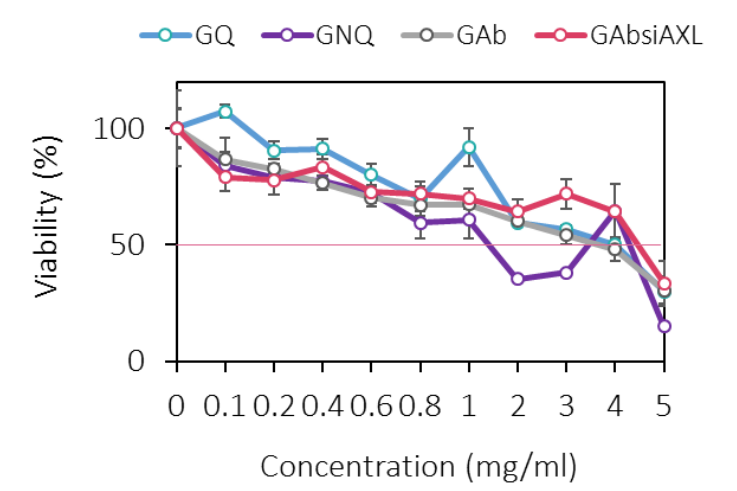

b

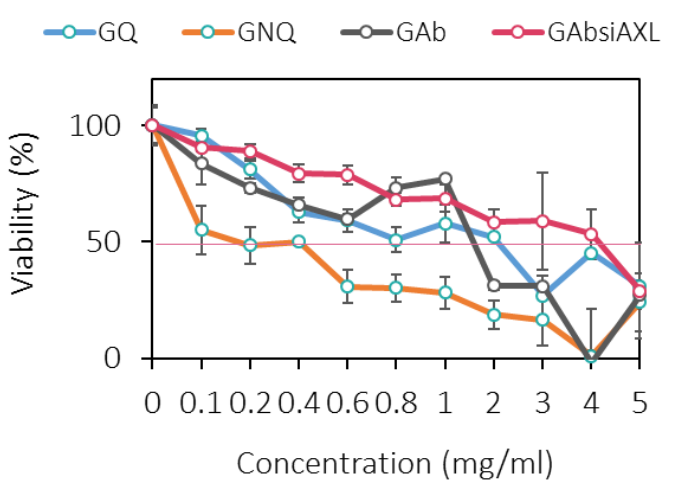

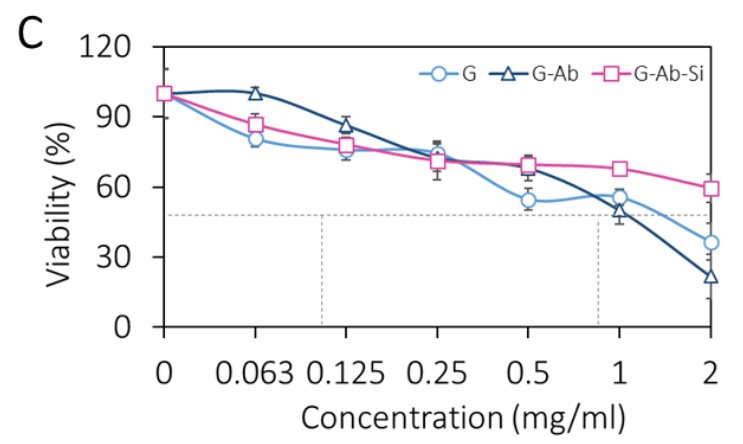

Figure 134. Toxicity profile for siRNA conjugated nanoparticles in (a) $24 \mathrm{~h}$ data in H820, (b) $24 \mathrm{~h}$ data in HCC 827 and (c) $72 \mathrm{~h}$ data in H820 cells. Results were averaged from 3 independent experiments. Based on the viability assay, $0.6 \mathrm{mg} / \mathrm{ml}$ was used as a treatment concentration for a period of $4 \mathrm{~h}$ in most MTT assays.

Cytotoxicity assays also confirmed that synthesized siRNA conjugates were non-toxic (Figure 134). As a next step we investigated drug resensitization in H820 cells using dual-siRNA AXL-FN14 constructs. Similar results to that of siRNA based treatments were achieved (Figure 135). The results demonstrate that nanoparticles can be utilized for targeting these pathways and can be an important step in translating therapies in higher organisms. 

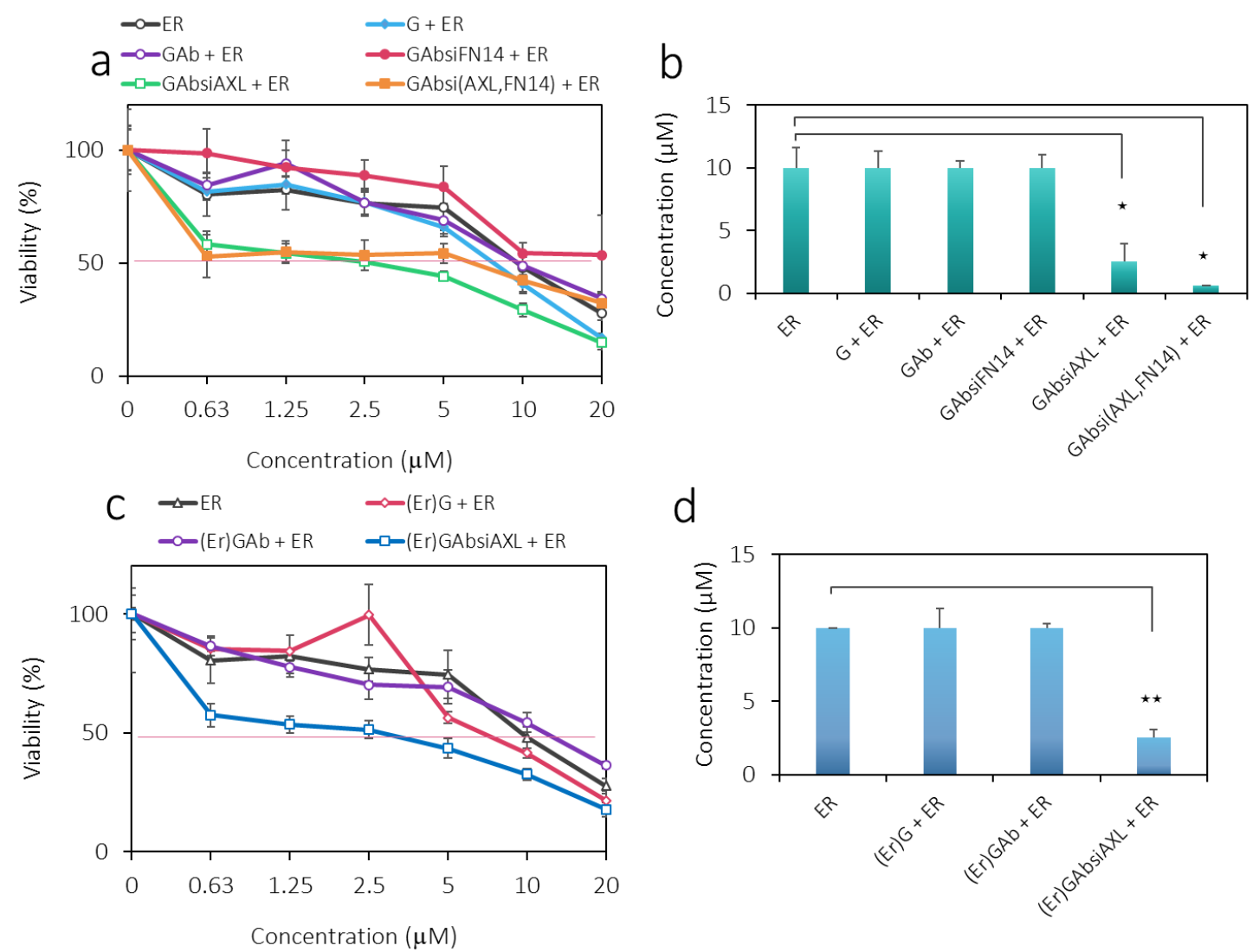

Figure 135. 72 h toxicity profile for synergistic drug and (a) siRNA conjugated nanoparticles and (a) erlotinib encapsulated siRNA constructs in H820 cells. Results were averaged from 3 independent experiments. ${ }^{\star} \mathrm{p} \leq 0.05$, ${ }^{\star} \mathrm{p} \leq$ 0.01 , and the values were analyzed using one-way anova.

\subsubsection{Targeting AXL-FN14 using nanoparticles in A549 mice xenografts}

In order to translate the in vitro findings to an in vivo model, we treated mice with luciferase expressing A549 xenograft tumors with our siRNA conjugated constructs. We synergistically targeted both AXL and FN14 using a single construct along with erlotinib and monitored tumor volume reduction by fluorescence imaging (Figure 136). Results indicated that targeted treatment using siRNA-constructs injected intraperitonealy, reduced the tumor size by $50 \%$ with time (at the third treatment day) as compared to nanoparticle devoid of siRNA (Figure 137). Controls treated with mice 
such as PBS or drug showed either a rapid increase or minimal reduction in tumor size (Figure 137).

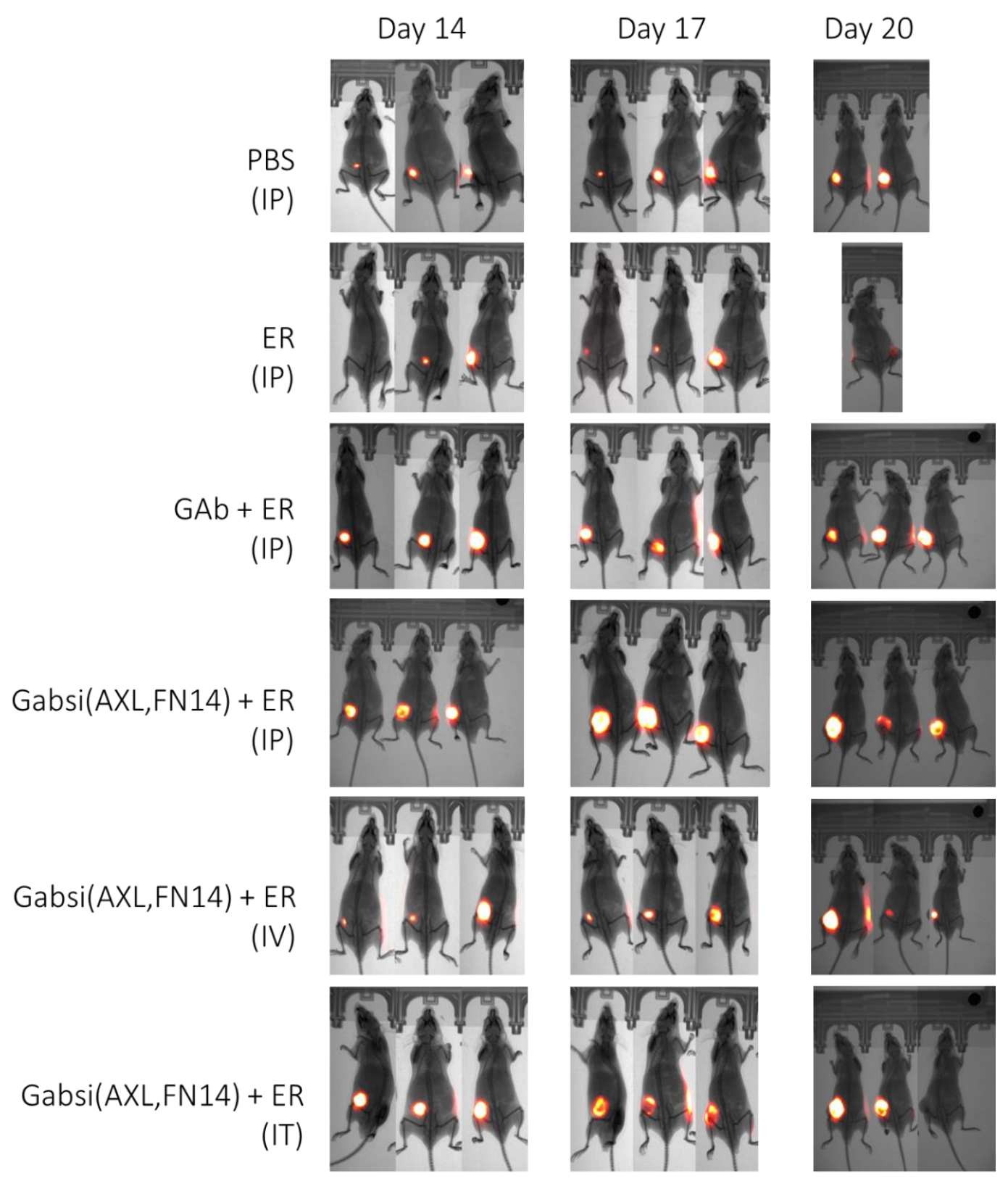

Figure 136. Fluorescence imaging of mice treated with constructs and drug. 
To confirm the downregulation of AXL-FN14 in tumors, protein analysis was performed on the tumor tissues. AXL and FN14 was found to be downregulated in tumor tissues treated with dual-siRNA construct GAbsi(AXL,FN14) as compared to
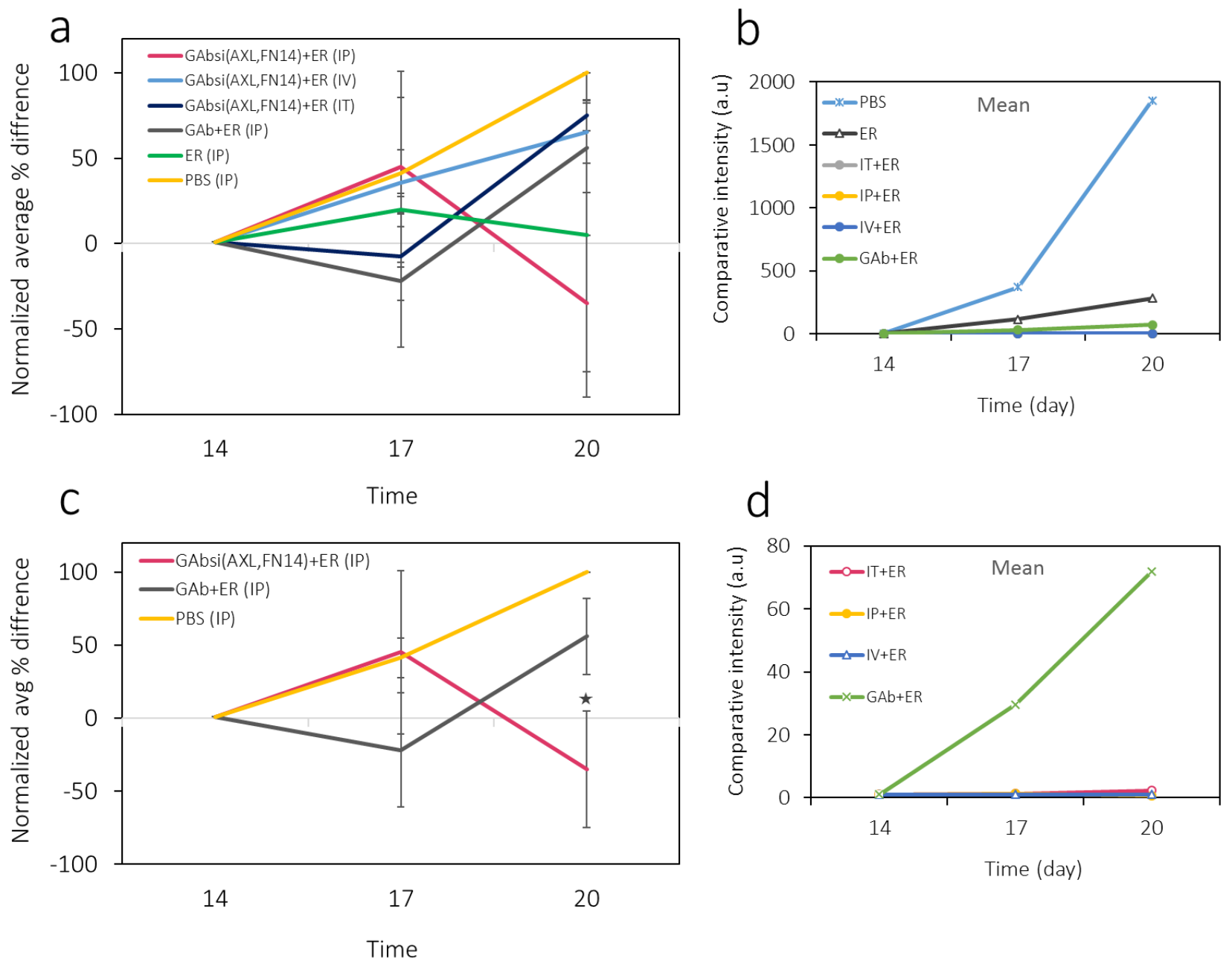

Figure 137. $(\mathrm{a}, \mathrm{c})$ Total fluorescence measured from tumors and (b,d) mean fluorescence measured from tumors. IT/IP/IV+ER represent dual-siRNA construct treatments. Results were averaged from 5 mice. ${ }^{\star} \mathrm{p} \leq 0.05$, and the normalized percentage differences between day 17 and day 20 values were analyzed using one-way anova.

controls (Figure 138). These results confirm resensitization of drug resistant tumors towards erlotinib (TKI) therapy. The in vivo studies demonstrate the use of nanoparticles for siRNA delivery to tumors, and drug resensitization. 

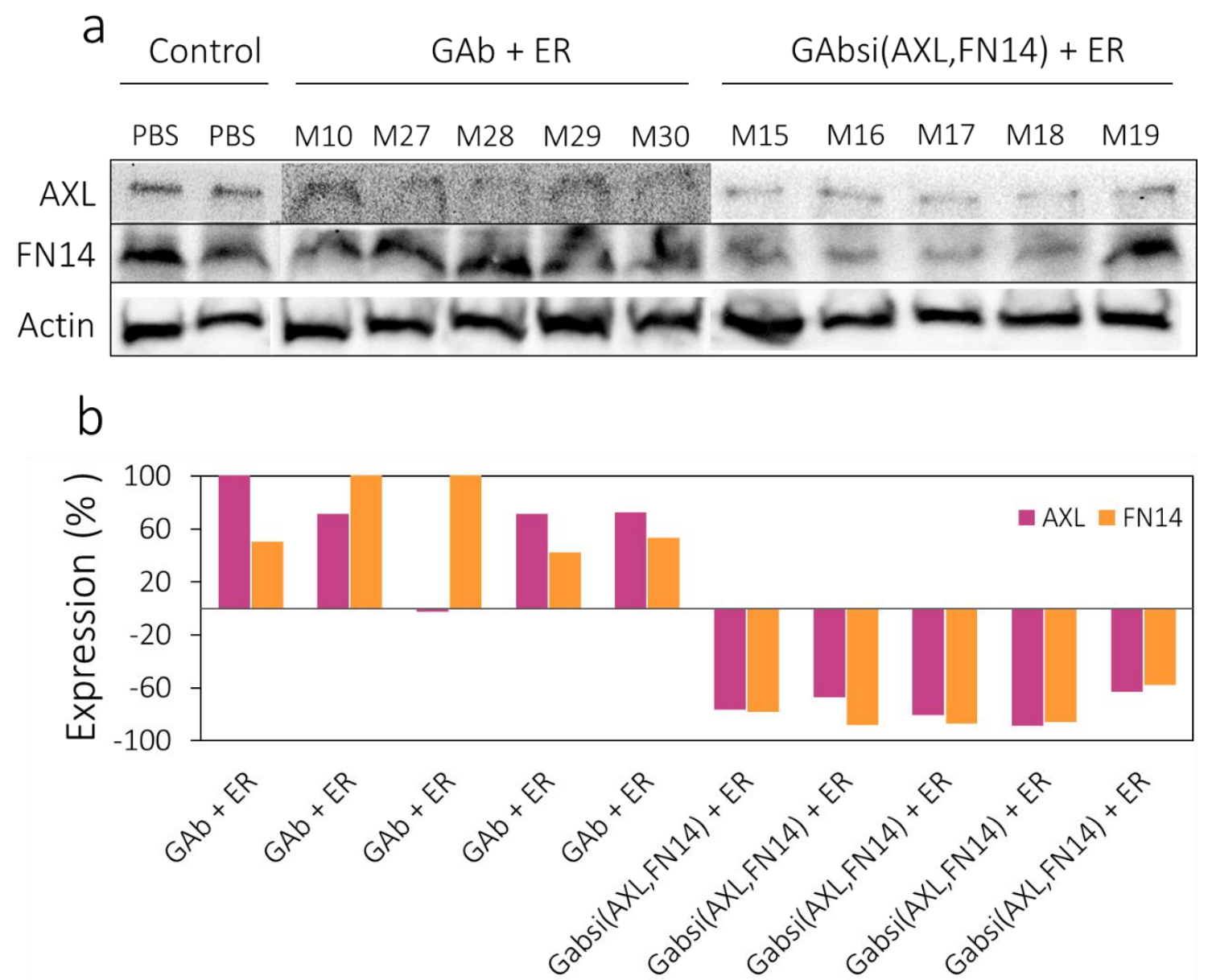

Figure 138. (a) Protein blot for AXL and FN14 expression in mice tumor samples and (b) band densitometry analysis for the blot by normalizing values to PBS controls. Results for PBS, GAb+ER and GAbsi(AXL,FN14)+ER were averaged from 2, 5 and 5 mice tumors respectively. Results show AXL and FN14 expression is drastically reduced in siRNA conjugated gelatin nanoparticle treatment samples.

\subsubsection{Impact of gene therapy in cancer}

Based on the in vitro and in vivo toxicity results that are solely based on temporary downregulation using siRNA as a mediator, we investigated resensitization after AXL gene knockout. For this study, we measured the $\mathrm{IC}_{50}$ of erlotinib in $\mathrm{H} 820 \mathrm{AK}$, 


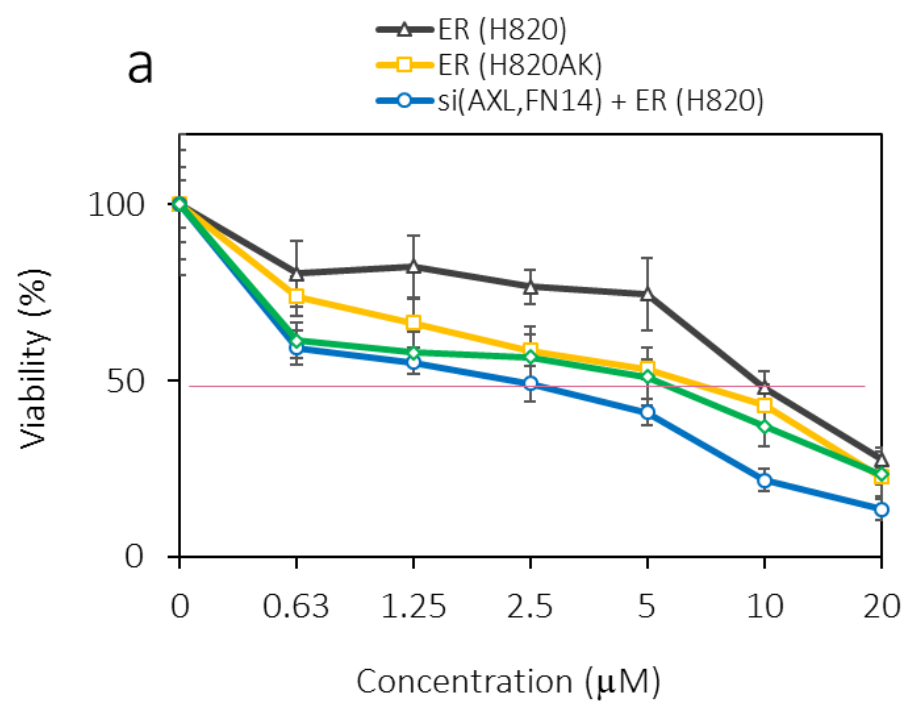

b

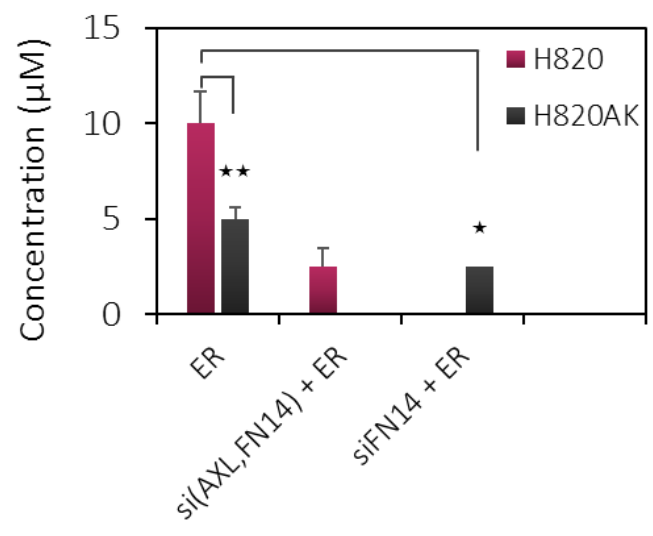

Figure 139. Resensitization to erlotinib in CRISPR assited AXL kncockout H820AK cell line for $72 \mathrm{~h}$. (a) Represents the cell viability plot and (b) represent the associated $\mathrm{IC}_{50}$ analysis. Results were averaged from 3 independent experiments. indicate $\mathrm{p} \leq 0.05,{ }^{\star \star} \mathrm{p} \leq 0.01$, and the values were analyzed using one-way anova..

wherein the AXL gene was non-functional. The results from $\mathrm{H} 820 \mathrm{AK}$ show that $\mathrm{IC}_{50}$ decreased by half (Figure 139). We could further resensitize H820AK by downregulating FN14 in these cells (Figure 139). Resensitization of these cells indicate the importance of synergistic treatment to target multiple pathways rather than a single pathway. Synergistic treatment perhaps can slow down compensatory mechanism and decrease treatment time. The result also suggests a basis for gene therapy in cancer patients whose post-treatment survival time is not significant Several ongoing research in animals have also shown promising results using this genetic engineering technique ${ }^{316-319}$. However, there is still much to be improved in targeted delivery of CRISPR components. Nevertheless, our results demonstrate that CRISPR based therapeutics has potential to genetic modify disease states within cells.

The in vitro and in vivo results strongly suggest the role of AXL oncogene in proliferation activities by regulating invasive and migration based pathways. This in 
turn might be increasing cell growth kinetics and allowing an increase of ATP concentration within the cell to competitively bind to EGFR tyrosine kinase domain against erlotinib and thereby increasing resistance. Additionally, AXL regulation of downstream targets might be influencing miRNA assisted resistance mechanisms as well. Several studies have also shown increased energy production in cancer cells were correlated with higher drug efflux through membrane pumps ${ }^{210}$. However, further exploration is required to understand and validate these complex signaling mechanisms.

\subsection{CONCLUSION}

Antibody conjugated gelatin nanoparticles effectively targets EGFR receptors on drug resistant NSCLC cells. Targeted delivery of siRNA specific to AXL or FN14, or both were evaluated in cells. The downregulation of pathways related to AXL in multiple drug resistant NSCLC has been studied in detail. Our data provide evidence of a survival crosstalk with FN14 post AXL knockdown. Similar results were seen after knocking the AXL gene in H820 cells. In vitro and in vivo studies in NSCLC cell and mouse models confirm AXL-FN14 cross-talk and drug resensitization upon knockdown. Additionally, this present study validates stable delivery of siRNA stably into cells using nanoparticles.

Overall, the study establishes AXL as an important oncogene that influences acquired drug resistance in NSCLC. The mechanisms suggested in this study can help develop new treatment strategies to combat drug resistance in cancer. 


\section{IMPLICATIONS OF THESE STUDIES}

The results of these studies emphasize nanoparticles as an innovative tool for both diagnostic and therapeutic approaches in cancer. Results show nanoparticles exhibit different properties based on their shape, constituency and surface modifications. These properties can be applied towards not only cancer research, but in all spheres of scientific applications. For example, functional materials that interact with external energy sources can be manipulated not only for medicinal reasons but also in opto-electronics, plasmonic metamaterials that use surface plasmon polariton (SPP) modes, and in atomic-scale maskless lithography required for nextgeneration atomic-scale circuit miniaturization. On the other hand, functional nanoparticles can be used as highly sensitive sensors to detect ctDNA or exosome for cancer diagnosis or as ultrasensitive chemical sensors. Additionally, the mechanisms unraveled in these studies need not be specific but may be generally suitable in other applications. For example, intracellular fate of nanoparticles can be manipulated by targeting different receptors using different surface targeting agents. Consequently, we can also design nanoparticles to target intracellular organelles for efficient therapeutics. Nanoparticles can be used to deliver agents like siRNA that have limited stability in bio-fluids. We can target various biochemical mechanics within the cell for therapeutic reasons, and understand the molecular biology of intercellular interactions. The results from the study also highlights the importance of gene therapy as a new approach towards cancer treatment. Overall, the data produced in these studies highlight the versatility of nanoparticles and provide information for developing better applications in the future. 


\section{REFERENCES}

1. Cheng, Y. et al. Highly efficient drug delivery with gold nanoparticle vectors for in vivo photodynamic therapy of cancer. J Am Chem Soc 130, 10643-10647 (2008).

2. Lajunen, T. et al. Light induced cytosolic drug delivery from liposomes with gold nanoparticles. $J$ Control Release 203, 85-98 (2015).

3. Yan, J. et al. Growth and origami folding of DNA on nanoparticles for high-efficiency molecular transport in cellular imaging and drug delivery. Angew Chem Int Ed Engl 54, 2431-2435 (2015).

4. Khlebtsov, N. \& Dykman, L. Biodistribution and toxicity of engineered gold nanoparticles: a review of in vitro and in vivo studies. Chem Soc Rev 40, 1647-1671 (2011).

5. Johnston, H.J. et al. A review of the in vivo and in vitro toxicity of silver and gold particulates: particle attributes and biological mechanisms responsible for the observed toxicity. Crit Rev Toxicol 40, 328-346 (2010).

6. Petros, R.A. \& DeSimone, J.M. Strategies in the design of nanoparticles for therapeutic applications. Nat Rev Drug Discov 9, 615-627 (2010).

7. Dutt, M. \& Khuller, G.K. Liposomes and PLG microparticles as sustained release antitubercular drug carriers--an in vitro-in vivo study. Int J Antimicrob Agents 18, 245-252 (2001).

8. Housman, G. et al. Drug resistance in cancer: an overview. Cancers (Basel) 6, 1769-1792 (2014).

9. Li, Z. et al. pH- and NIR Light-Responsive Polymeric Prodrug Micelles for Hyperthermia-Assisted Site-Specific Chemotherapy to Reverse Drug Resistance in Cancer Treatment. Small 12, 27312740 (2016).

10. Albanese, A., Tang, P.S. \& Chan, W.C. The effect of nanoparticle size, shape, and surface chemistry on biological systems. Annu Rev Biomed Eng 14, 1-16 (2012).

11. Win, K.Y. \& Feng, S.S. Effects of particle size and surface coating on cellular uptake of polymeric nanoparticles for oral delivery of anticancer drugs. Biomaterials 26, 2713-2722 (2005). 
12. Wolfe, B.L. \& Trejo, J. Clathrin-dependent mechanisms of G protein-coupled receptor endocytosis. Traffic 8, 462-470 (2007).

13. Rejman, J., Oberle, V., Zuhorn, I.S. \& Hoekstra, D. Size-dependent internalization of particles via the pathways of clathrin- and caveolae-mediated endocytosis. Biochem J 377, 159-169 (2004).

14. Bonifacino, J.S. \& Traub, L.M. Signals for sorting of transmembrane proteins to endosomes and lysosomes. Annu Rev Biochem 72, 395-447 (2003).

15. Zhou, K. et al. Tunable, ultrasensitive $\mathrm{pH}$-responsive nanoparticles targeting specific endocytic organelles in living cells. Angew Chem Int Ed Engl 50, 6109-6114 (2011).

16. Lasagna-Reeves, C. et al. Bioaccumulation and toxicity of gold nanoparticles after repeated administration in mice. Biochem Biophys Res Commun 393, 649-655 (2010).

17. Heo, D.N. et al. Gold nanoparticles surface-functionalized with paclitaxel drug and biotin receptor as theranostic agents for cancer therapy. Biomaterials 33, 856-866 (2012).

18. Melancon, M.P. et al. In vitro and in vivo targeting of hollow gold nanoshells directed at epidermal growth factor receptor for photothermal ablation therapy. Mol Cancer Ther 7, 1730-1739 (2008).

19. Chen, J. et al. Facile synthesis of gold-silver nanocages with controllable pores on the surface. $J$ Am Chem Soc 128, 14776-14777 (2006).

20. You, J. et al. Photothermal-chemotherapy with doxorubicin-loaded hollow gold nanospheres: A platform for near-infrared light-trigged drug release. J Control Release 158, 319-328 (2012).

21. Zheng, M. \& Yu, J. The effect of particle shape and size on cellular uptake. Drug Deliv Transl Res 6, 67-72 (2016).

22. Li, Y., Kroger, M. \& Liu, W.K. Shape effect in cellular uptake of PEGylated nanoparticles: comparison between sphere, rod, cube and disk. Nanoscale 7, 16631-16646 (2015).

23. Bar-Shavit, R. et al. G Protein-Coupled Receptors in Cancer. Int J Mol Sci 17 (2016).

24. Dorsam, R.T. \& Gutkind, J.S. G-protein-coupled receptors and cancer. Nat Rev Cancer 7, 79-94 (2007). 
25. Smith, C.J. et al. Radiochemical investigations of 177Lu-DOTA-8-Aoc-BBN[7-14]NH2: an in vitro/in vivo assessment of the targeting ability of this new radiopharmaceutical for PC-3 human prostate cancer cells. Nucl Med Biol 30, 101-109 (2003).

26. Yang, P.H., Sun, X., Chiu, J.F., Sun, H. \& He, Q.Y. Transferrin-mediated gold nanoparticle cellular uptake. Bioconjug Chem 16, 494-496 (2005).

27. Bhattacharyya, S. et al. Switching the targeting pathways of a therapeutic antibody by nanodesign. Angew Chem Int Ed Engl 51, 1563-1567 (2012).

28. Li, P.C. et al. In vivo photoacoustic molecular imaging with simultaneous multiple selective targeting using antibody-conjugated gold nanorods. Opt Express 16, 18605-18615 (2008).

29. Choi, C.H., Alabi, C.A., Webster, P. \& Davis, M.E. Mechanism of active targeting in solid tumors with transferrin-containing gold nanoparticles. Proc Natl Acad Sci U S A 107, 1235-1240 (2010).

30. Dhar, S., Daniel, W.L., Giljohann, D.A., Mirkin, C.A. \& Lippard, S.J. Polyvalent oligonucleotide gold nanoparticle conjugates as delivery vehicles for platinum(IV) warheads. J Am Chem Soc 131, $14652-14653$ (2009).

31. Chithrani, B.D., Ghazani, A.A. \& Chan, W.C. Determining the size and shape dependence of gold nanoparticle uptake into mammalian cells. Nano Lett 6, 662-668 (2006).

32. Tkachenko, A.G. et al. Cellular trajectories of peptide-modified gold particle complexes: comparison of nuclear localization signals and peptide transduction domains. Bioconjug Chem 15, 482-490 (2004).

33. Levy, R. et al. Rational and combinatorial design of peptide capping ligands for gold nanoparticles. J Am Chem Soc 126, 10076-10084 (2004).

34. Chanda, N. et al. Bombesin functionalized gold nanoparticles show in vitro and in vivo cancer receptor specificity. Proc Natl Acad Sci U S A 107, 8760-8765 (2010).

35. Yuan, H., Fales, A.M. \& Vo-Dinh, T. TAT peptide-functionalized gold nanostars: enhanced intracellular delivery and efficient NIR photothermal therapy using ultralow irradiance. J Am Chem Soc 134, 11358-11361 (2012). 
36. Oyelere, A.K., Chen, P.C., Huang, X., El-Sayed, I.H. \& El-Sayed, M.A. Peptide-conjugated gold nanorods for nuclear targeting. Bioconjug Chem 18, 1490-1497 (2007).

37. Prades, R. et al. Delivery of gold nanoparticles to the brain by conjugation with a peptide that recognizes the transferrin receptor. Biomaterials 33, 7194-7205 (2012).

38. Yang, Y.S., Zhang, X., Xiong, Z. \& Chen, X. Comparative in vitro and in vivo evaluation of two 64Cu-labeled bombesin analogs in a mouse model of human prostate adenocarcinoma. Nucl Med Biol 33, 371-380 (2006).

39. Skrabalak, S.E. et al. Gold nanocages: synthesis, properties, and applications. Acc Chem Res 41, 1587-1595 (2008).

40. Mitchell, H., Choudhury, A., Pagano, R.E. \& Leof, E.B. Ligand-dependent and -independent transforming growth factor-beta receptor recycling regulated by clathrin-mediated endocytosis and Rab11. Mol Biol Cell 15, 4166-4178 (2004).

41. Bagwe, R.P., Hilliard, L.R. \& Tan, W. Surface modification of silica nanoparticles to reduce aggregation and nonspecific binding. Langmuir 22, 4357-4362 (2006).

42. Bland, J.M. \& Altman, D.G. Calculating correlation coefficients with repeated observations: Part 1--Correlation within subjects. BMJ 310, 446 (1995).

43. Podsiadlo, P. et al. Gold nanoparticles enhance the anti-leukemia action of a 6-mercaptopurine chemotherapeutic agent. Langmuir 24, 568-574 (2008).

44. Reile, H., Armatis, P.E. \& Schally, A.V. Characterization of high-affinity receptors for bombesin/gastrin releasing peptide on the human prostate cancer cell lines PC-3 and DU-145: internalization of receptor bound 125I-(Tyr4) bombesin by tumor cells. Prostate 25, 29-38 (1994).

45. Ming, X. et al. Intracellular delivery of an antisense oligonucleotide via endocytosis of a G proteincoupled receptor. Nucleic Acids Res 38, 6567-6576 (2010).

46. Casibang, M. \& Moody, T.W. (Tyr(0),Bpa(4))bombesin is a GRP receptor agonist. Peptides 21, 649-653 (2000). 
47. Wang, L. et al. Selective targeting of gold nanorods at the mitochondria of cancer cells: implications for cancer therapy. Nano Lett 11, 772-780 (2011).

48. Yang, L., Shang, L. \& Nienhaus, G.U. Mechanistic aspects of fluorescent gold nanocluster internalization by live HeLa cells. Nanoscale 5, 1537-1543 (2013).

49. Saha, K. et al. Surface functionality of nanoparticles determines cellular uptake mechanisms in mammalian cells. Small 9, 300-305 (2013).

50. Hailstones, D., Sleer, L.S., Parton, R.G. \& Stanley, K.K. Regulation of caveolin and caveolae by cholesterol in MDCK cells. J Lipid Res 39, 369-379 (1998).

51. Zhao, S. et al. Monitoring the transport of polymeric micelles across MDCK cell monolayer and exploring related mechanisms. J Control Release 158, 413-423 (2012).

52. Brodin, L., Low, P. \& Shupliakov, O. Sequential steps in clathrin-mediated synaptic vesicle endocytosis. Curr Opin Neurobiol 10, 312-320 (2000).

53. Mittal, N. et al. Select G-protein-coupled receptors modulate agonist-induced signaling via a ROCK, LIMK, and beta-arrestin 1 pathway. Cell Rep 5, 1010-1021 (2013).

54. Schumann, M., Nakagawa, T., Mantey, S.A., Howell, B. \& Jensen, R.T. Function of non-visual arrestins in signaling and endocytosis of the gastrin-releasing peptide receptor (GRP receptor). Biochem Pharmacol 75, 1170-1185 (2008).

55. Ungewickell, E.J. \& Hinrichsen, L. Endocytosis: clathrin-mediated membrane budding. Curr Opin Cell Biol 19, 417-425 (2007).

56. Le Roy, C. \& Wrana, J.L. Clathrin- and non-clathrin-mediated endocytic regulation of cell signalling. Nat Rev Mol Cell Biol 6, 112-126 (2005).

57. Tan, C.M., Brady, A.E., Nickols, H.H., Wang, Q. \& Limbird, L.E. Membrane trafficking of G protein-coupled receptors. Annu Rev Pharmacol Toxicol 44, 559-609 (2004).

58. Chu, Z. et al. Unambiguous observation of shape effects on cellular fate of nanoparticles. Sci Rep 4, 4495 (2014). 
59. Ellis, P.M. \& Vandermeer, R. Delays in the diagnosis of lung cancer. J Thorac Dis 3, 183-188 (2011).

60. La Thangue, N.B. \& Kerr, D.J. Predictive biomarkers: a paradigm shift towards personalized cancer medicine. Nat Rev Clin Oncol 8, 587-596 (2011).

61. Pepe, M.S. et al. Phases of biomarker development for early detection of cancer. J Natl Cancer Inst 93, 1054-1061 (2001).

62. Shigematsu, H. et al. Clinical and biological features associated with epidermal growth factor receptor gene mutations in lung cancers. J Natl Cancer Inst 97, 339-346 (2005).

63. Wallander, M.L., Geiersbach, K.B., Tripp, S.R. \& Layfield, L.J. Comparison of reverse transcription-polymerase chain reaction, immunohistochemistry, and fluorescence in situ hybridization methodologies for detection of echinoderm microtubule-associated proteinlike 4anaplastic lymphoma kinase fusion-positive non-small cell lung carcinoma: implications for optimal clinical testing. Arch Pathol Lab Med 136, 796-803 (2012).

64. Brunnstrom, H. et al. Immunohistochemistry in the differential diagnostics of primary lung cancer: an investigation within the Southern Swedish Lung Cancer Study. Am J Clin Pathol 140, 37-46 (2013).

65. Bubendorf, L. et al. Multitarget FISH analysis in the diagnosis of lung cancer. Am J Clin Pathol 123, 516-523 (2005).

66. Wang, J. et al. Quantifying EGFR alterations in the lung cancer genome with nanofluidic digital PCR arrays. Clin Chem 56, 623-632 (2010).

67. Okumura, Y. et al. Circulating tumor cells in pulmonary venous blood of primary lung cancer patients. Ann Thorac Surg 87, 1669-1675 (2009).

68. Rolfo, C. et al. Liquid biopsies in lung cancer: the new ambrosia of researchers. Biochim Biophys Acta 1846, 539-546 (2014).

69. Maheswaran, S. \& Haber, D.A. Circulating tumor cells: a window into cancer biology and metastasis. Curr Opin Genet Dev 20, 96-99 (2010). 
70. Schneck, H. et al. EpCAM-Independent Enrichment of Circulating Tumor Cells in Metastatic Breast Cancer. Plos One 10, e0144535 (2015).

71. Aktas, B. et al. Stem cell and epithelial-mesenchymal transition markers are frequently overexpressed in circulating tumor cells of metastatic breast cancer patients. Breast Cancer Res 11, R46 (2009).

72. Gradilone, A. et al. Circulating tumour cells lacking cytokeratin in breast cancer: the importance of being mesenchymal. J Cell Mol Med 15, 1066-1070 (2011).

73. Krebs, M.G. et al. Analysis of circulating tumor cells in patients with non-small cell lung cancer using epithelial marker-dependent and -independent approaches. J Thorac Oncol 7, 306-315 (2012).

74. Brabletz, T., Jung, A., Spaderna, S., Hlubek, F. \& Kirchner, T. Opinion: migrating cancer stem cells - an integrated concept of malignant tumour progression. Nat Rev Cancer 5, 744-749 (2005).

75. De Craene, B. \& Berx, G. Regulatory networks defining EMT during cancer initiation and progression. Nat Rev Cancer 13, 97-110 (2013).

76. Vuoriluoto, K. et al. Vimentin regulates EMT induction by Slug and oncogenic H-Ras and migration by governing Axl expression in breast cancer. Oncogene 30, 1436-1448 (2011).

77. Page-McCaw, A., Ewald, A.J. \& Werb, Z. Matrix metalloproteinases and the regulation of tissue remodelling. Nat Rev Mol Cell Biol 8, 221-233 (2007).

78. Polette, M., Nawrocki-Raby, B., Gilles, C., Clavel, C. \& Birembaut, P. Tumour invasion and matrix metalloproteinases. Crit Rev Oncol Hematol 49, 179-186 (2004).

79. Singh, A. \& Settleman, J. EMT, cancer stem cells and drug resistance: an emerging axis of evil in the war on cancer. Oncogene 29, 4741-4751 (2010).

80. Nurwidya, F., Takahashi, F., Murakami, A. \& Takahashi, K. Epithelial mesenchymal transition in drug resistance and metastasis of lung cancer. Cancer Res Treat 44, 151-156 (2012). 
81. Zuo, J.H. et al. Activation of EGFR promotes squamous carcinoma SCC10A cell migration and invasion via inducing EMT-like phenotype change and MMP-9-mediated degradation of Ecadherin. J Cell Biochem 112, 2508-2517 (2011).

82. Zhang, Z. et al. Activation of the AXL kinase causes resistance to EGFR-targeted therapy in lung cancer. Nat Genet 44, 852-860 (2012).

83. Huang, D. et al. Cisplatin resistance in gastric cancer cells is associated with HER2 upregulationinduced epithelial-mesenchymal transition. Sci Rep 6, 20502 (2016).

84. Huang, L. \& Fu, L. Mechanisms of resistance to EGFR tyrosine kinase inhibitors. Acta Pharm Sin $B$ 5, 390-401 (2015).

85. Stewart, E.L., Tan, S.Z., Liu, G. \& Tsao, M.S. Known and putative mechanisms of resistance to EGFR targeted therapies in NSCLC patients with EGFR mutations-a review. Transl Lung Cancer $\operatorname{Res}$ 4, 67-81 (2015).

86. Colomiere, M. et al. Cross talk of signals between EGFR and IL-6R through JAK2/STAT3 mediate epithelial-mesenchymal transition in ovarian carcinomas. Br J Cancer 100, 134-144 (2009).

87. Chung, S.S., Giehl, N., Wu, Y. \& Vadgama, J.V. STAT3 activation in HER2-overexpressing breast cancer promotes epithelial-mesenchymal transition and cancer stem cell traits. Int J Oncol 44, 403411 (2014).

88. Ludwig, J.A. \& Weinstein, J.N. Biomarkers in cancer staging, prognosis and treatment selection. Nature Reviews Cancer 5, 845-856 (2005).

89. Kim, D. et al. Synthesis of uniform ferrimagnetic magnetite nanocubes. J Am Chem Soc 131, 454455 (2009).

90. Guardia, P. et al. Water-soluble iron oxide nanocubes with high values of specific absorption rate for cancer cell hyperthermia treatment. ACS Nano 6, 3080-3091 (2012).

91. Salado, J. et al. Functionalized $\mathrm{Fe}(3) \mathrm{O}(4) @ \mathrm{Au}$ superparamagnetic nanoparticles: in vitro bioactivity. Nanotechnology 23, 315102 (2012). 
92. Morrison, G. \& Loye, H.C.Z. Simple correction for the sample shape and radial offset effects on SQUID magnetometers: Magnetic measurements on $\operatorname{Ln}(2) \mathrm{O}(3)(\mathrm{Ln}=\mathrm{Gd}$, Dy, Er) standards. $J$ Solid State Chem 221, 334-337 (2015).

93. Li, D. et al. BIBW2992, an irreversible EGFR/HER2 inhibitor highly effective in preclinical lung cancer models. Oncogene 27, 4702-4711 (2008).

94. Giannopoulou, E. et al. Epidermal growth factor receptor status and Notch inhibition in non-small cell lung cancer cells. J Biomed Sci 22, 98 (2015).

95. Hyun, K.A. et al. Epithelial-to-mesenchymal transition leads to loss of EpCAM and different physical properties in circulating tumor cells from metastatic breast cancer. Oncotarget 7, 24677 24687 (2016).

96. Blume, G. \& Cevc, G. Liposomes for the sustained drug release in vivo. Biochim Biophys Acta 1029, 91-97 (1990).

97. Ungaro, F., De Rosa, G., Miro, A., Quaglia, F. \& La Rotonda, M.I. Cyclodextrins in the production of large porous particles: development of dry powders for the sustained release of insulin to the lungs. Eur J Pharm Sci 28, 423-432 (2006).

98. Leroux, J.C., Allemann, E., DeJaeghere, F., Doelker, E. \& Gurny, R. Biodegradable nanoparticles - From sustained release formulations to improved site specific drug delivery. Journal of Controlled Release 39, 339-350 (1996).

99. Elvira, C. et al. Evaluation of drug delivery characteristics of microspheres of PMMA-PCLcholesterol obtained by supercritical-CO2 impregnation and by dissolution-evaporation techniques. J Control Release 99, 231-240 (2004).

100. Wei, H., Zhang, X., Cheng, C., Cheng, S.X. \& Zhuo, R.X. Self-assembled, thermosensitive micelles of a star block copolymer based on PMMA and PNIPAAm for controlled drug delivery. Biomaterials 28, 99-107 (2007).

101. You, Y.Z., Kalebaila, K.K., Brock, S.L. \& Oupicky, D. Temperature-controlled uptake and release in PNIPAM-modified porous silica nanoparticles. Chem Mater 20, 3354-3359 (2008). 
102. Bhattarai, N., Gunn, J. \& Zhang, M. Chitosan-based hydrogels for controlled, localized drug delivery. Adv Drug Deliv Rev 62, 83-99 (2010).

103. Lee, C.F., Wen, C.J., Lin, C.L. \& Chiu, W.Y. Morphology and temperature responsivenessswelling relationship of poly( $\mathrm{N}$-isopropylamide-chitosan) copolymers and their application to drug release. J Polym Sci Pol Chem 42, 3029-3037 (2004).

104. Cooperstein, M.A. \& Canavan, H.E. Assessment of cytotoxicity of (N-isopropyl acrylamide) and poly(N-isopropyl acrylamide)-coated surfaces. Biointerphases 8, 19 (2013).

105. Husseini, G.A. \& Pitt, W.G. Micelles and nanoparticles for ultrasonic drug and gene delivery. Adv Drug Deliv Rev 60, 1137-1152 (2008).

106. Yao, J.H., Feng, J.X. \& Chen, J. External-stimuli responsive systems for cancer theranostic. Asian J Pharm Sci 11, 585-595 (2016).

107. Timko, B.P. et al. Near-infrared-actuated devices for remotely controlled drug delivery. P Natl Acad Sci USA 111, 1349-1354 (2014).

108. Jain, P.K., Huang, X., El-Sayed, I.H. \& El-Sayed, M.A. Noble metals on the nanoscale: optical and photothermal properties and some applications in imaging, sensing, biology, and medicine. Acc Chem Res 41, 1578-1586 (2008).

109. Zhou, Z.Y., Tian, N., Li, J.T., Broadwell, I. \& Sun, S.G. Nanomaterials of high surface energy with exceptional properties in catalysis and energy storage. Chem Soc Rev 40, 4167-4185 (2011).

110. Ghosh, S.K. \& Pal, T. Interparticle coupling effect on the surface plasmon resonance of gold nanoparticles: from theory to applications. Chem Rev 107, 4797-4862 (2007).

111. Grieve, K., Mulvaney, P. \& Grieser, F. Synthesis and electronic properties of semiconductor nanoparticles/quantum dots. Curr Opin Colloid In 5, 168-172 (2000).

112. Zhang, H. et al. Growth of manganese oxide nanoflowers on vertically-aligned carbon nanotube arrays for high-rate electrochemical capacitive energy storage. Nano Lett 8, 2664-2668 (2008). 
113. Frey, N.A., Peng, S., Cheng, K. \& Sun, S.H. Magnetic nanoparticles: synthesis, functionalization, and applications in bioimaging and magnetic energy storage. Chemical Society Reviews 38, 25322542 (2009).

114. Lee, S.F. et al. Ultrasound, pH, and magnetically responsive crown-ether-coated core/shell nanoparticles as drug encapsulation and release systems. ACS Appl Mater Interfaces 5, 1566-1574 (2013).

115. Huang, S.L. \& MacDonald, R.C. Acoustically active liposomes for drug encapsulation and ultrasound-triggered release. Bba-Biomembranes 1665, 134-141 (2004).

116. Yang, J.P. et al. Spatially Confined Fabrication of Core-Shell Gold Nanocages@ Mesoporous Silica for Near-Infrared Controlled Photothermal Drug Release. Chem Mater 25, 3030-3037 (2013).

117. Letfullin, R.R., Joenathan, C., George, T.F. \& Zharov, V.P. Laser-induced explosion of gold nanoparticles: potential role for nanophotothermolysis of cancer. Nanomedicine (Lond) 1, 473-480 (2006).

118. Qin, Z.P. \& Bischof, J.C. Thermophysical and biological responses of gold nanoparticle laser heating. Chemical Society Reviews 41, 1191-1217 (2012).

119. Govorov, A.O. \& Richardson, H.H. Generating heat with metal nanoparticles. Nano Today 2, 3038 (2007).

120. Huang, X., El-Sayed, I.H., Qian, W. \& El-Sayed, M.A. Cancer cell imaging and photothermal therapy in the near-infrared region by using gold nanorods. J Am Chem Soc 128, 2115-2120 (2006).

121. Baek, S.W. et al. Au@Ag core-shell nanocubes for efficient plasmonic light scattering effect in low bandgap organic solar cells. ACS Nano 8, 3302-3312 (2014).

122. Cheng, L. et al. PEGylated Prussian blue nanocubes as a theranostic agent for simultaneous cancer imaging and photothermal therapy. Biomaterials 35, 9844-9852 (2014).

123. Sherry, L.J. et al. Localized surface plasmon resonance spectroscopy of single silver nanocubes. Nano Lett 5, 2034-2038 (2005). 
124. Pelton, M., Liu, M.Z., Park, S., Scherer, N.F. \& Guyot-Sionnest, P. Ultrafast resonant optical scattering from single gold nanorods: Large nonlinearities and plasmon saturation. Phys Rev B $\mathbf{7 3}$ (2006).

125. Wei, G., Wang, J. \& Chen, Y. Electromagnetic enhancement of ordered silver nanorod arrays evaluated by discrete dipole approximation. Beilstein J Nanotechnol 6, 686-696 (2015).

126. Grubisic, A. et al. Plasmonic Near-Electric Field Enhancement Effects in Ultrafast Photoelectron Emission: Correlated Spatial and Laser Polarization Microscopy Studies of Individual Ag Nanocubes. Nano Letters 12, 4823-4829 (2012).

127. Stehle, C., Zimmermann, C. \& Slama, S. Cooperative coupling of ultracold atoms and surface plasmons. Nat Phys 10, 937-942 (2014).

128. Biener, J. et al. Surface Chemistry in Nanoscale Materials. Materials 2, 2404-2428 (2009).

129. Rao, C.N., Kulkarni, G.U., Thomas, P.J. \& Edwards, P.P. Size-dependent chemistry: properties of nanocrystals. Chemistry 8, 28-35 (2002).

130. Eustis, S. \& El-Sayed, M.A. Why gold nanoparticles are more precious than pretty gold: Noble metal surface plasmon resonance and its enhancement of the radiative and nonradiative properties of nanocrystals of different shapes. Chemical Society Reviews 35, 209-217 (2006).

131. Link, S., Wang, Z.L. \& El-Sayed, M.A. Alloy formation of gold-silver nanoparticles and the dependence of the plasmon absorption on their composition. J Phys Chem B 103, 3529-3533 (1999).

132. Jain, P.K., Lee, K.S., El-Sayed, I.H. \& El-Sayed, M.A. Calculated absorption and scattering properties of gold nanoparticles of different size, shape, and composition: Applications in biological imaging and biomedicine. J Phys Chem B 110, 7238-7248 (2006).

133. Link, S. \& El-Sayed, M.A. Size and temperature dependence of the plasmon absorption of colloidal gold nanoparticles. J Phys Chem B 103, 4212-4217 (1999). 
134. Li, X.D., Chen, T.P., Liu, Y. \& Leong, K.C. Influence of localized surface plasmon resonance and free electrons on the optical properties of ultrathin Au films: a study of the aggregation effect. Optics Express 22, 5124-5132 (2014).

135. Hu, M. et al. Dark-field microscopy studies of single metal nanoparticles: understanding the factors that influence the linewidth of the localized surface plasmon resonance. J Mater Chem 18, 19491960 (2008).

136. Funston, A.M., Novo, C., Davis, T.J. \& Mulvaney, P. Plasmon Coupling of Gold Nanorods at Short Distances and in Different Geometries. Nano Letters 9, 1651-1658 (2009).

137. Ming, T. et al. Strong polarization dependence of plasmon-enhanced fluorescence on single gold nanorods. Nano Lett 9, 3896-3903 (2009).

138. Liaw, J.W., Cheng, J.C., Ma, C.M. \& Zhang, R.F. Theoretical Analysis of Plasmon Modes of AuAg Nanocages. J Phys Chem C 117, 19586-19592 (2013).

139. Hazra, B. \& Chandra, M. Plasmon Hybridization Mediated Structure-Specific Refractive Index Sensitivity of Hollow Gold Nanoprism in the Vis-NIR Region. Acs Sensors 1, 536-542 (2016).

140. Mahmoud, M.A. \& El-Sayed, M.A. Metallic double shell hollow nanocages: the challenges of their synthetic techniques. Langmuir 28, 4051-4059 (2012).

141. Mahmoud, M.A. \& El-Sayed, M.A. Gold Nanoframes: Very High Surface Plasmon Fields and Excellent Near-Infrared Sensors. Journal of the American Chemical Society 132, 12704-12710 (2010).

142. Preciado-Flores, S. et al. Highly reproducible synthesis of hollow gold nanospheres with near infrared surface plasmon absorption using PVP as stabilizing agent. J Mater Chem 21, 2344-2350 (2011).

143. Yavuz, M.S. et al. Gold nanocages covered by smart polymers for controlled release with nearinfrared light. Nat Mater 8, 935-939 (2009).

144. Chen, J. et al. Immuno gold nanocages with tailored optical properties for targeted photothermal destruction of cancer cells. Nano Lett 7, 1318-1322 (2007). 
145. Thomsen, S. Pathologic analysis of photothermal and photomechanical effects of laser-tissue interactions. Photochem Photobiol 53, 825-835 (1991).

146. Hu, M. et al. Ultrafast laser studies of the photothermal properties of gold nanocages. $J$ Phys Chem $B$ 110, 1520-1524 (2006).

147. Gamaly, E.G. \& Rode, A.V. Physics of ultra-short laser interaction with matter: From phonon excitation to ultimate transformations. Prog Quant Electron 37, 215-323 (2013).

148. Gamaly, E.G. The physics of ultra-short laser interaction with solids at non-relativistic intensities. Phys Rep 508, 91-243 (2011).

149. Grua, P., Morreeuw, J.P., Bercegol, H., Jonusauskas, G. \& Vallee, F. Electron kinetics and emission for metal nanoparticles exposed to intense laser pulses. Phys Rev B 68 (2003).

150. Link, S., Burda, C., Wang, Z.L. \& El-Sayed, M.A. Electron dynamics in gold and gold-silver alloy nanoparticles: The influence of a nonequilibrium electron distribution and the size dependence of the electron-phonon relaxation. J Chem Phys 111, 1255-1264 (1999).

151. Mitrani, J.M., Shneider, M.N., Stratton, B.C. \& Raitses, Y. Modeling thermionic emission from laser-heated nanoparticles. Appl Phys Lett 108 (2016).

152. Link, S., Burda, C., Nikoobakht, B. \& El-Sayed, M.A. Laser-induced shape changes of colloidal gold nanorods using femtosecond and nanosecond laser pulses. J Phys Chem B 104, 6152-6163 (2000).

153. Inasawa, S., Sugiyama, M. \& Yamaguchi, Y. Laser-induced shape transformation of gold nanoparticles below the melting point: the effect of surface melting. $J$ Phys Chem $B$ 109, 3104$3111(2005)$.

154. Rethfeld, B., Kaiser, A., Vicanek, M. \& Simon, G. Ultrafast dynamics of nonequilibrium electrons in metals under femtosecond laser irradiation. Phys Rev B 65 (2002).

155. Ashcroft, N.W. \& Mermin, N.D. Solid State Physics. (Holt, Rinehart and Winston, 1976).

156. Plech, A., Kotaidis, V., Lorenc, M. \& Boneberg, J. Femtosecond laser near-field ablation from gold nanoparticles. Nat Phys 2, 44-47 (2006). 
157. Sun, Q. et al. Direct imaging of the near field and dynamics of surface plasmon resonance on gold nanostructures using photoemission electron microscopy. Light-Sci Appl 2 (2013).

158. Chen, X.L., Munjiza, A., Zhang, K. \& Wen, D.S. Molecular Dynamics Simulation of Heat Transfer from a Gold Nanoparticle to a Water Pool. J Phys Chem C 118, 1285-1293 (2014).

159. Albrecht, W. et al. Single Particle Deformation and Analysis of Silica-Coated Gold Nanorods before and after Femtosecond Laser Pulse Excitation. Nano Letters 16, 1818-1825 (2016).

160. Raman, R.K. et al. Electronically Driven Fragmentation of Silver Nanocrystals Revealed by Ultrafast Electron Crystallography. Phys Rev Lett 104 (2010).

161. Zhang, Q. et al. Production of Ag nanocubes on a scale of $0.1 \mathrm{~g}$ per batch by protecting the NaHSmediated polyol synthesis with argon. ACS Appl Mater Interfaces 1, 2044-2048 (2009).

162. Haiss, W., Thanh, N.T., Aveyard, J. \& Fernig, D.G. Determination of size and concentration of gold nanoparticles from UV-vis spectra. Anal Chem 79, 4215-4221 (2007).

163. Solomon, S.D. et al. Synthesis and study of silver nanoparticles. J Chem Educ 84, 322-325 (2007).

164. Noguez, C. Surface Plasmons on Metal Nanoparticles: The Influence of Shape and Physical Environment. J Phys Chem C 111, 3806-3819 (2007).

165. Li, Z.Y. \& Xia, Y. Metal nanoparticles with gain toward single-molecule detection by surfaceenhanced Raman scattering. Nano Lett 10, 243-249 (2010).

166. Akman, E., Oztoprak, B.G., Gunes, M., Kacar, E. \& Demir, A. Effect of femtosecond Ti:Sapphire laser wavelengths on plasmonic behaviour and size evolution of silver nanoparticles. Photonic Nanostruct 9, 276-286 (2011).

167. Wang, F. et al. Interactions between individual carbon nanotubes studied by Rayleigh scattering spectroscopy. Phys Rev Lett 96 (2006).

168. Besner, S. \& Meunier, M. Femtosecond Laser Synthesis of AuAg Nanoalloys: Photoinduced Oxidation and Ions Release. J Phys Chem C 114, 10403-10409 (2010).

169. Herbani, Y., Nakamura, T. \& Sato, S. Synthesis of platinum-based binary and ternary alloy nanoparticles in an intense laser field. J Colloid Interf Sci 375, 78-87 (2012). 
170. Qian, J. et al. Effect of Edge Rounding on the Extinction Properties of Hollow Metal Nanoparticles. Plasmonics 8, 955-962 (2013).

171. Huang, H. et al. Controlled joining of Ag nanoparticles with femtosecond laser radiation. J Appl Phys 112 (2012).

172. Pustovalov, V.K., Smetannikov, A.S. \& Zharov, V.P. Photothermal and accompanied phenomena of selective nanophotothermolysis with gold nanoparticles and laser pulses. Laser Phys Lett 5, 775792 (2008).

173. Uramoto, H., Shimokawa, H., Hanagiri, T., Kuwano, M. \& Ono, M. Expression of selected gene for acquired drug resistance to EGFR-TKI in lung adenocarcinoma. Lung Cancer 73, 361-365 (2011).

174. Balak, M.N. et al. Novel D761Y and common secondary T790M mutations in epidermal growth factor receptor-mutant lung adenocarcinomas with acquired resistance to kinase inhibitors. Clin Cancer Res 12, 6494-6501 (2006).

175. Maruyama, R., Wataya, H., Seto, T. \& Ichinose, Y. Treatment after the failure of gefitinib in patients with advanced or recurrent non-small cell lung cancer. Anticancer Res 29, 4217-4221 (2009).

176. Brown, R., Curry, E., Magnani, L., Wilhelm-Benartzi, C.S. \& Borley, J. Poised epigenetic states and acquired drug resistance in cancer. Nature Reviews Cancer 14, 747-753 (2014).

177. Godwin, A.K. et al. High-Resistance to Cisplatin in Human Ovarian-Cancer Cell-Lines Is Associated with Marked Increase of Glutathione Synthesis. P Natl Acad Sci USA 89, 3070-3074 (1992).

178. Ma, C., Wei, S. \& Song, Y. T790M and acquired resistance of EGFR TKI: a literature review of clinical reports. J Thorac Dis 3, 10-18 (2011).

179. Lynch, T.J. et al. Activating mutations in the epidermal growth factor receptor underlying responsiveness of non-small-cell lung cancer to gefitinib. N Engl J Med 350, 2129-2139 (2004). 
180. Allred, D.C. et al. HER-2/neu in node-negative breast cancer: prognostic significance of overexpression influenced by the presence of in situ carcinoma. J Clin Oncol 10, 599-605 (1992).

181. Alessi, P. et al. Anti-FGF2 approaches as a strategy to compensate resistance to anti-VEGF therapy: long-pentraxin 3 as a novel antiangiogenic FGF2-antagonist. Eur Cytokine Netw 20, 225-234 (2009).

182. Witton, C.J., Reeves, J.R., Going, J.J., Cooke, T.G. \& Bartlett, J.M. Expression of the HER1-4 family of receptor tyrosine kinases in breast cancer. J Pathol 200, 290-297 (2003).

183. Kiyokawa, E. et al. Overexpression of Erk, an Eph Family Receptor Protein-Tyrosine Kinase, in Various Human Tumors. Cancer Res 54, 3645-3650 (1994).

184. Lutz, M.P. et al. Overexpression and activation of the tyrosine kinase Src in human pancreatic carcinoma. Biochem Bioph Res Co 243, 503-508 (1998).

185. Yarden, Y. The EGFR family and its ligands in human cancer. signalling mechanisms and therapeutic opportunities. Eur J Cancer 37 Suppl 4, S3-8 (2001).

186. De Luca, A. et al. The role of the EGFR signaling in tumor microenvironment. J Cell Physiol 214, 559-567 (2008).

187. Janda, E., Litos, G., Grunert, S., Downward, J. \& Beug, H. Oncogenic Ras/Her-2 mediate hyperproliferation of polarized epithelial cells in 3D cultures and rapid tumor growth via the PI3K pathway. Oncogene 21, 5148-5159 (2002).

188. Son, J. et al. Glutamine supports pancreatic cancer growth through a KRAS-regulated metabolic pathway (vol 496, pg 101, 2013). Nature 499 (2013).

189. Aiello, M. et al. Role of genetic polymorphisms and mutations in colorectal cancer therapy (Review). Mol Med Rep 4, 203-208 (2011).

190. de Mello, R.A. et al. EGFR and KRAS mutations, and ALK fusions: current developments and personalized therapies for patients with advanced non-small-cell lung cancer. Pharmacogenomics 14, 1765-1777 (2013). 
191. Tol, J., Nagtegaal, I.D. \& Punt, C.J.A. BRAF Mutation in Metastatic Colorectal Cancer. New Engl J Med 361, 98-99 (2009).

192. Marusyk, A., Almendro, V. \& Polyak, K. Intra-tumour heterogeneity: a looking glass for cancer? Nature Reviews Cancer 12, 323-334 (2012).

193. Errico, A. Lung cancer: Heterogeneity in space and time. Nat Rev Clin Oncol 11, 684 (2014).

194. Inoue, Y. et al. Phase II study of erlotinib in elderly patients with non-small cell lung cancer harboring epidermal growth factor receptor mutations. Cancer Chemother Pharmacol 76, 155-161 (2015).

195. Thress, K.S. et al. Acquired EGFR C797S mutation mediates resistance to AZD9291 in non-small cell lung cancer harboring EGFR T790M. Nat Med 21, 560-562 (2015).

196. Looyenga, B.D., Cherni, I., MacKeigan, J.P. \& Weiss, G.J. Tailoring Tyrosine Kinase Inhibitors to Fit the Lung Cancer Genome. Transl Oncol 4, 59-70 (2011).

197. Rosell, R. et al. Erlotinib versus standard chemotherapy as first-line treatment for European patients with advanced EGFR mutation-positive non-small-cell lung cancer (EURTAC): a multicentre, open-label, randomised phase 3 trial. Lancet Oncol 13, 239-246 (2012).

198. Thatcher, N. et al. Gefitinib plus best supportive care in previously treated patients with refractory advanced non-small-cell lung cancer: results from a randomised, placebo-controlled, multicentre study (Iressa Survival Evaluation in Lung Cancer). Lancet 366, 1527-1537 (2005).

199. Cataldo, V.D., Gibbons, D.L., Perez-Soler, R. \& Quintas-Cardama, A. Treatment of non-small-cell lung cancer with erlotinib or gefitinib. N Engl J Med 364, 947-955 (2011).

200. Wang, J., Wang, B.C., Chu, H.L. \& Yao, Y.F. Intrinsic resistance to EGFR tyrosine kinase inhibitors in advanced non-small-cell lung cancer with activating EGFR mutations. Oncotargets Ther 9, 3711-3726 (2016).

201. Rolfo, C. et al. Improvement in Lung Cancer Outcomes With Targeted Therapies: An Update for Family Physicians. J Am Board Fam Med 28, 124-133 (2015). 
202. Gettinger, S. et al. Nivolumab Monotherapy for First-Line Treatment of Advanced Non-Small-Cell Lung Cancer. Journal of Clinical Oncology 34, 2980-+ (2016).

203. Xu, M., Xie, Y., Ni, S. \& Liu, H. The latest therapeutic strategies after resistance to first generation epidermal growth factor receptor tyrosine kinase inhibitors (EGFR TKIs) in patients with non-small cell lung cancer (NSCLC). Ann Transl Med 3, 96 (2015).

204. Xu, Y., Liu, H.Y., Chen, J. \& Zhou, Q.H. Acquired resistance of lung adenocarcinoma to EGFRtyrosine kinase inhibitors gefitinib and erlotinib. Cancer Biol Ther 9, 572-582 (2010).

205. Giovannetti, E. et al. Molecular mechanisms underlying the synergistic interaction of erlotinib, an epidermal growth factor receptor tyrosine kinase inhibitor, with the multitargeted antifolate pemetrexed in non-small-cell lung cancer cells. Mol Pharmacol 73, 1290-1300 (2008).

206. Tsvetkova, E. \& Goss, G.D. Drug resistance and its significance for treatment decisions in nonsmall-cell lung cancer. Curr Oncol 19, S45-S51 (2012).

207. Tetsu, O., Phuchareon, J., Eisele, D.W. \& McCormick, F. ETS1 inactivation causes innate drug resistance to EGFR inhibitors. Mol Cell Oncol 3 (2016).

208. Mancini, M. \& Yarden, Y. Mutational and network level mechanisms underlying resistance to anticancer kinase inhibitors. Semin Cell Dev Biol 50, 164-176 (2016).

209. Ko, R. et al. Frequency of EGFR T790M mutation and multimutational profiles of rebiopsy samples from non-small cell lung cancer developing acquired resistance to EGFR tyrosine kinase inhibitors in Japanese patients. Bmc Cancer 16 (2016).

210. Gottesman, M.M. Mechanisms of cancer drug resistance. Annu Rev Med 53, 615-627 (2002).

211. Oxnard, G.R. et al. Acquired Resistance to EGFR Tyrosine Kinase Inhibitors in EGFR-Mutant Lung Cancer: Distinct Natural History of Patients with Tumors Harboring the T790M Mutation. Clinical Cancer Research 17, 1616-1622 (2011).

212. Yu, H.A. et al. Analysis of Tumor Specimens at the Time of Acquired Resistance to EGFR-TKI Therapy in 155 Patients with EGFR-Mutant Lung Cancers. Clinical Cancer Research 19, 22402247 (2013). 
213. Kuo, C.H. et al. Subsequent Chemotherapy Improves Survival Outcome in Advanced Non-SmallCell Lung Cancer With Acquired Tyrosine Kinase Inhibitor Resistance. Clin Lung Cancer 11, 51$56(2010)$.

214. Quail, D.F. \& Joyce, J.A. Microenvironmental regulation of tumor progression and metastasis. Nature Medicine 19, 1423-1437 (2013).

215. Miller, M.A. et al. Reduced Proteolytic Shedding of Receptor Tyrosine Kinases Is a PostTranslational Mechanism of Kinase Inhibitor Resistance. Cancer Discov 6, 382-399 (2016).

216. Miller, M.A. et al. ADAM-10 and-17 regulate endometriotic cell migration via concerted ligand and receptor shedding feedback on kinase signaling. P Natl Acad Sci USA 110, E2074-E2083 (2013).

217. Wu, X.L. et al. AXL kinase as a novel target for cancer therapy. Oncotarget 5, 9546-9576 (2014).

218. Song, X.Z. et al. Overexpression of Receptor Tyrosine Kinase Axl Promotes Tumor Cell Invasion and Survival in Pancreatic Ductal Adenocarcinoma. Cancer-Am Cancer Soc 117, 734-743 (2011).

219. Ahmed, L. et al. Increased tumor cell expression of Axl is a marker of aggressive features in breast cancer among African women. Apmis 123, 688-696 (2015).

220. Liu, R. et al. Induction, regulation, and biologic function of Axl receptor tyrosine kinase in Kaposi sarcoma. Blood 116, 297-305 (2010).

221. Ruan, G.X. \& Kazlauskas, A. Axl is essential for VEGF-A-dependent activation of PI3K/Akt. Embo J 31, 1692-1703 (2012).

222. Lee, W.P., Wen, Y., Varnum, B. \& Hung, M.C. Akt is required for Axl-Gas6 signaling to protect cells from E1A-mediated apoptosis. Oncogene 21, 329-336 (2002).

223. Tai, K.Y., Shieh, Y.S., Lee, C.S., Shiah, S.G. \& Wu, C.W. Axl promotes cell invasion by inducing MMP-9 activity through activation of NF-kappa B and Brg-1. Oncogene 27, 4044-4055 (2008).

224. Asiedu, M.K. et al. AXL induces epithelial-to-mesenchymal transition and regulates the function of breast cancer stem cells. Oncogene 33, 1316-1324 (2014). 
225. Lamouille, S., Xu, J. \& Derynck, R. Molecular mechanisms of epithelial-mesenchymal transition. Nat Rev Mol Cell Bio 15, 178-196 (2014).

226. Khan, K.H., Yap, T.A., Yan, L. \& Cunningham, D. Targeting the PI3K-AKT-mTOR signaling network in cancer. Chin J Cancer 32, 253-265 (2013).

227. Elkabets, M. et al. AXL mediates resistance to PI3Kalpha inhibition by activating the EGFR/PKC/mTOR axis in head and neck and esophageal squamous cell carcinomas. Cancer Cell 27, 533-546 (2015).

228. Uramoto, H. et al. Epithelial-Mesenchymal Transition in EGFR-TKI Acquired Resistant Lung Adenocarcinoma. Anticancer Research 30, 2513-2517 (2010).

229. Soutschek, J. et al. Therapeutic silencing of an endogenous gene by systemic administration of modified siRNAs. Nature 432, 173-178 (2004).

230. Filipowicz, W., Jaskiewicz, L., Kolb, F.A. \& Pillai, R.S. Post-transcriptional gene silencing by siRNAs and miRNAs. Curr Opin Struc Biol 15, 331-341 (2005).

231. Sorensen, D.R., Leirdal, M. \& Sioud, M. Gene silencing by systemic delivery of synthetic siRNAs in adult mice. J Mol Biol 327, 761-766 (2003).

232. Engels, J.W. Gene silencing by chemically modified siRNAs. New Biotechnol 30, 302-307 (2013).

233. Faehnle, C.R., Elkayam, E., Haase, A.D., Hannon, G.J. \& Joshua-Tor, L. Slicer and the Argonautes. Biophys J 106, 455a-455a (2014).

234. Filipowicz, W. RNAi: The nuts and bolts of the RISC machine. Cell 122, 17-20 (2005).

235. Roberts, T.C., Ezzat, K., El Andaloussi, S. \& Weinberg, M.S. Synthetic SiRNA Delivery: Progress and Prospects. Methods Mol Biol 1364, 291-310 (2016).

236. Lee, H.S. et al. Contributions of 3'-overhang to the dissociation of small interfering RNAs from the PAZ domain: molecular dynamics simulation study. J Mol Graph Model 25, 784-793 (2007).

237. Li, Y.Y. et al. Axl Mediates Tumor Invasion and Chemosensitivity Through PI3K/Akt Signaling Pathway and Is Transcriptionally Regulated by Slug in Breast Carcinoma. Iubmb Life 66, 507-518 (2014). 
238. Doudna, J.A. \& Charpentier, E. The new frontier of genome engineering with CRISPR-Cas9. Science 346, 1077-+ (2014).

239. Ran, F.A. et al. Genome engineering using the CRISPR-Cas9 system. Nat Protoc 8, 2281-2308 (2013).

240. Ran, F.A. et al. Double Nicking by RNA-Guided CRISPR Cas9 for Enhanced Genome Editing Specificity. Cell 154, 1380-1389 (2013).

241. Shalem, O. et al. Genome-Scale CRISPR-Cas9 Knockout Screening in Human Cells. Science 343, 84-87 (2014).

242. Zhang, H. et al. The CRISPR/Cas9 system produces specific and homozygous targeted gene editing in rice in one generation. Plant Biotechnol J 12, 797-807 (2014).

243. Gratz, S.J. et al. Highly Specific and Efficient CRISPR/Cas9-Catalyzed Homology-Directed Repair in Drosophila. Genetics 196, 961-+ (2014).

244. Bale, A.S. et al. Correlating In Vitro Data to In Vivo Findings for Risk Assessment. Altex-Altern Anim Ex 31, 79-90 (2014).

245. Williams, C.S. et al. Celecoxib prevents tumor growth in vivo without toxicity to normal gut: Lack of correlation between in vitro and in vivo models. Cancer Res 60, 6045-+ (2000).

246. Maeda, H. Vascular permeability in cancer and infection as related to macromolecular drug delivery, with emphasis on the EPR effect for tumor-selective drug targeting. P Jpn Acad B-Phys 88, 53-71 (2012).

247. Maeda, H., Nakamura, H. \& Fang, J. Nanomedicine for Cancer Selective Drug Delivery; Advantages of the Epr Effect and Pitfalls. Anticancer Research 34, 6042-6044 (2014).

248. Maeda, H. The Epr Effect and Beyond for Cancer Selective Drug Delivery for Treatment and Imaging Using Nanomedicine. Drug Metab Rev 45, 15-15 (2014).

249. Maeda, H., Nakamura, H. \& Fang, J. The EPR effect for macromolecular drug delivery to solid tumors: Improvement of tumor uptake, lowering of systemic toxicity, and distinct tumor imaging in vivo. Adv Drug Deliver Rev 65, 71-79 (2013). 
250. Malam, Y., Loizidou, M. \& Seifalian, A.M. Liposomes and nanoparticles: nanosized vehicles for drug delivery in cancer. Trends Pharmacol Sci 30, 592-599 (2009).

251. Acharya, S. \& Sahoo, S.K. PLGA nanoparticles containing various anticancer agents and tumour delivery by EPR effect. Adv Drug Deliver Rev 63, 170-183 (2011).

252. Kaul, G. \& Amiji, M. Tumor-targeted gene delivery using poly(ethylene glycol)-modified gelatin nanoparticles: In vitro and in vivo studies. Pharm Res 22, 951-961 (2005).

253. Tseng, C.L. et al. Development of gelatin nanoparticles with biotinylated EGF conjugation for lung cancer targeting. Biomaterials 28, 3996-4005 (2007).

254. Yu, C. et al. Novel aptamer-nanoparticle bioconjugates enhances delivery of anticancer drug to MUC1-positive cancer cells in vitro. Plos One 6, e24077 (2011).

255. Whitehead, K.A., Langer, R. \& Anderson, D.G. Knocking down barriers: advances in siRNA delivery. Nature Reviews Drug Discovery 8, 129-138 (2009).

256. Dokka, S., Toledo, D., Shi, X.G., Castranova, V. \& Rojanasakul, Y. Oxygen radical-mediated pulmonary toxicity induced by some cationic liposomes. Pharm Res 17, 521-525 (2000).

257. Srikar, R. et al. Targeted nanoconjugate co-delivering siRNA and tyrosine kinase inhibitor to KRAS mutant NSCLC dissociates GAB1-SHP2 post oncogene knockdown. Sci Rep 6, 30245 (2016).

258. Bigi, A., Cojazzi, G., Panzavolta, S., Rubini, K. \& Roveri, N. Mechanical and thermal properties of gelatin films at different degrees of glutaraldehyde crosslinking. Biomaterials 22, 763-768 (2001).

259. Van Vlierberghe, S., Vanderleyden, E., Boterberg, V. \& Dubruel, P. Gelatin Functionalization of Biomaterial Surfaces: Strategies for Immobilization and Visualization. Polymers-Basel 3, 114-130 (2011).

260. Bartczak, D. \& Kanaras, A.G. Preparation of Peptide-Functionalized Gold Nanoparticles Using One Pot EDC/Sulfo-NHS Coupling. Langmuir 27, 10119-10123 (2011). 
261. Baumer, N. et al. Antibody-coupled siRNA as an efficient method for in vivo mRNA knockdown. Nat Protoc 11, 22-36 (2016).

262. Srikar, R., Suresh, D., Saranathan, S., Zambre, A. \& Kannan, R. Three-Dimensional Nanocomposites: Fluidics Driven Assembly of Metal Nanoparticles on Protein Nanostructures and Their Cell-Line-Dependent Intracellular Trafficking Pattern. Langmuir 32, 4877-4885 (2016).

263. Bartlett, D.W. \& Davis, M.E. Insights into the kinetics of siRNA-mediated gene silencing from live-cell and live-animal bioluminescent imaging. Nucleic Acids Research 34, 322-333 (2006).

264. Feng, Z. et al. The regulation of AMPK beta1, TSC2, and PTEN expression by p53: stress, cell and tissue specificity, and the role of these gene products in modulating the IGF-1-AKT-mTOR pathways. Cancer Res 67, 3043-3053 (2007).

265. Feng, Z., Zhang, H., Levine, A.J. \& Jin, S. The coordinate regulation of the p53 and mTOR pathways in cells. Proc Natl Acad Sci U S A 102, 8204-8209 (2005).

266. Boysen, J. et al. The tumor suppressor axis p53/miR-34a regulates Axl expression in B-cell chronic lymphocytic leukemia: implications for therapy in p53-defective CLL patients. Leukemia 28, 451455 (2014).

267. Mouraviev, V. et al. Clinical prospects of long noncoding RNAs as novel biomarkers and therapeutic targets in prostate cancer. Prostate Cancer P D 19, 14-20 (2016).

268. Li, G.B. et al. Long Noncoding RNA Plays a Key Role in Metastasis and Prognosis of Hepatocellular Carcinoma. Biomed Res Int (2014).

269. Ronnau, C.G.H., Verhaegh, G.W., Luna-Velez, M.V. \& Schalken, J.A. Noncoding RNAs as Novel Biomarkers in Prostate Cancer. Biomed Res Int (2014).

270. Kutanzi, K.R., Yurchenko, O.V., Beland, F.A., Checkhun, V.F. \& Pogribny, I.P. MicroRNAmediated drug resistance in breast cancer. Clin Epigenetics 2, 171-185 (2011).

271. Garajova, I. et al. Molecular Mechanisms Underlying the Role of MicroRNAs in the Chemoresistance of Pancreatic Cancer. Biomed Res Int (2014). 
272. Han, F. et al. Emerging Roles of MicroRNAs in EGFR-Targeted Therapies for Lung Cancer. Biomed Res Int (2015).

273. Magne, N. et al. Influence of epidermal growth factor receptor (EGFR), p53 and intrinsic MAP kinase pathway status of tumour cells on the antiproliferative effect of ZD1839 ("Iressa"). $\mathrm{Br} J$ Cancer 86, 1518-1523 (2002).

274. Bheda, A., Creek, K.E. \& Pirisi, L. Loss of p53 induces epidermal growth factor receptor promoter activity in normal human keratinocytes. Oncogene 27, 4315-4323 (2008).

275. Meyer, A.S., Miller, M.A., Gertler, F.B. \& Lauffenburger, D.A. The Receptor AXL Diversifies EGFR Signaling and Limits the Response to EGFR-Targeted Inhibitors in Triple-Negative Breast Cancer Cells. Sci Signal 6 (2013).

276. Mudduluru, G., Vajkoczy, P. \& Allgayer, H. Myeloid Zinc Finger 1 Induces Migration, Invasion, and In vivo Metastasis through Axl Gene Expression in Solid Cancer. Mol Cancer Res 8, 159-169 (2010).

277. Merghoub, T. et al. Mzf1 controls cell proliferation and tumorigenesis. Blood 98, 97a-97a (2001).

278. Kolch, W., Halasz, M., Granovskaya, M. \& Kholodenko, B.N. The dynamic control of signal transduction networks in cancer cells. Nature Reviews Cancer 15, 515-527 (2015).

279. Chen, L. et al. MicroRNA-432 functions as a tumor suppressor gene through targeting E2F3 and AXL in lung adenocarcinoma. Oncotarget 7, 20041-20053 (2016).

280. Wang, Y. et al. Axl-altered microRNAs regulate tumorigenicity and gefitinib resistance in lung cancer. Cell Death Dis 5 (2014).

281. Xu, X.Y. et al. miR-374a promotes cell proliferation, migration and invasion by targeting SRCIN1 in gastric cancer. Febs Lett 589, 407-413 (2015).

282. Han, J. et al. Gas6/Axl mediates tumor cell apoptosis, migration and invasion and predicts the clinical outcome of osteosarcoma patients. Biochem Biophys Res Commun 435, 493-500 (2013).

283. Zhang, Y.X. et al. AXL is a potential target for therapeutic intervention in breast cancer progression. Cancer Res 68, 1905-1915 (2008). 
284. Toth, M. \& Fridman, R. Assessment of Gelatinases (MMP-2 and MMP-9 by Gelatin Zymography. Methods Mol Med 57, 163-174 (2001).

285. Rho, J.K. et al. MET and AXL Inhibitor NPS-1034 Exerts Efficacy against Lung Cancer Cells Resistant to EGFR Kinase Inhibitors Because of MET or AXL Activation. Cancer Res 74, 253-262 (2014).

286. Radisky, E.S. \& Radisky, D.C. Matrix Metalloproteinase-Induced Epithelial-Mesenchymal Transition in Breast Cancer. J Mammary Gland Biol 15, 201-212 (2010).

287. Tester, A.M., Ruangpanit, N., Anderson, R.L. \& Thompson, E.W. MMP-9 secretion and MMP-2 activation distinguish invasive and metastatic sublines of a mouse mammary carcinoma system showing epithelial-mesenchymal transition traits. Clin Exp Metastasis 18, 553-560 (2000).

288. Chiu, K.C. et al. Protumoral effect of macrophage through Axl activation on mucoepidermoid carcinoma. J Oral Pathol Med 43, 538-544 (2014).

289. Bonaldo, P. \& Sandri, M. Cellular and molecular mechanisms of muscle atrophy. Dis Model Mech 6, 25-39 (2013).

290. Wang, J., Ye, Q. \& She, Q.B. New insights into 4E-BP1-regulated translation in cancer progression and metastasis. Cancer Cell Microenviron 1 (2014).

291. Xiao, L., Wang, Y.C., Li, W.S. \& Du, Y. The role of mTOR and phospho-p70S6K in pathogenesis and progression of gastric carcinomas: an immunohistochemical study on tissue microarray. $J$ Exp Clin Canc Res 28 (2009).

292. Heiden, M.G.V., Cantley, L.C. \& Thompson, C.B. Understanding the Warburg Effect: The Metabolic Requirements of Cell Proliferation. Science 324, 1029-1033 (2009).

293. Cardnell, R.J. et al. Activation of the PI3K/mTOR Pathway following PARP Inhibition in Small Cell Lung Cancer. Plos One 11 (2016).

294. Mendoza, M.C., Er, E.E. \& Blenis, J. The Ras-ERK and PI3K-mTOR pathways: cross-talk and compensation. Trends Biochem Sci 36, 320-328 (2011). 
295. Girvan, A.C. et al. AGRO100 inhibits activation of nuclear factor-kappa B (NF-kappa B) by forming a complex with NF-kappa B essential modulator (NEMO) and nucleolin. Molecular Cancer Therapeutics 5, 1790-1799 (2006).

296. Tsou, J.H. et al. Nucleolin regulates c-Jun/Sp1-dependent transcriptional activation of cPLA(2)alpha in phorbol ester-treated non-small cell lung cancer A549 cells. Nucleic Acids Research 36, 217-227 (2008).

297. Whitsett, T.G. et al. Elevated expression of Fn14 in non-small cell lung cancer correlates with mutant KRAS or amplified MET and promotes tumor cell invasion and metastasis. Molecular Biology of the Cell 23 (2012).

298. Okamoto, K. et al. Role of Survivin in EGFR Inhibitor-Induced Apoptosis in Non-Small Cell Lung Cancers Positive for EGFR Mutations. Cancer Res 70, 10402-10410 (2010).

299. Winkles, J.A. The TWEAK - Fn14 cytokine-receptor axis: discovery, biology and therapeutic targeting. Nature Reviews Drug Discovery 7, 411-425 (2008).

300. Johnston, A.J. et al. Targeting of Fn14 Prevents Cancer-Induced Cachexia and Prolongs Survival. Cell 162, 1365-1378 (2015).

301. Whitsett, T.G. et al. Elevated Expression of Fn14 in Non-Small Cell Lung Cancer Correlates with Activated EGFR and Promotes Tumor Cell Migration and Invasion. Am J Pathol 181, 111-120 (2012).

302. Whitsett, T.G. et al. Mcl-1 Mediates TWEAK/Fn14-Induced Non-Small Cell Lung Cancer Survival and Therapeutic Response. Mol Cancer Res 12, 550-559 (2014).

303. Kwon, O.H., Kim, J.H., Kim, S.Y. \& Kim, Y.S. TWEAK/Fn14 signaling mediates gastric cancer cell resistance to 5-fluorouracil via NF-kappa B activation. International Journal of Oncology 44, 583-590 (2014).

304. Kohn, K.W. et al. Gene Expression Profiles of the NCI-60 Human Tumor Cell Lines Define Molecular Interaction Networks Governing Cell Migration Processes. Plos One 7 (2012). 
305. Enwere, E.K., LaCasse, E.C., Adam, N.J. \& Korneluk, R.G. Role of theTWEAK-Fn14-cIAP1-NFkappa B signaling axis in the regulation of myogenesis anc muscle homeostasis. Front Immunol $\mathbf{5}$ (2014).

306. Poveda, J. et al. Tweak/Fn14 and non-canonical NF-kappaB signaling in kidney disease. Front Immunol 4 (2013).

307. Brown, S.A.N., Cheng, E., Williams, M.S. \& Winkles, J.A. TWEAK-Independent Fn14 SelfAssociation and NF-kappa B Activation Is Mediated by the C-Terminal Region of the Fn14 Cytoplasmic Domain. Plos One 8 (2013).

308. Chen, S.X. et al. Fn14, a Downstream Target of the TGF-beta Signaling Pathway, Regulates Fibroblast Activation. Plos One 10 (2015).

309. Heldin, C.H., Miyazono, K. \& tenDijke, P. TGF-beta signalling from cell membrane to nucleus through SMAD proteins. Nature 390, 465-471 (1997).

310. Reichl, P. et al. Axl Activates Autocrine Transforming Growth Factor-beta Signaling in Hepatocellular Carcinoma. Hepatology 61, 930-941 (2015).

311. Ijichi, H. et al. Smad4-independent regulation of p21/WAF1 by transforming growth factor-beta. Oncogene 23, 1043-1051 (2004).

312. Das, T.P., Suman, S., Alatassi, H., Ankem, M.K. \& Damodaran, C. Inhibition of AKT promotes FOXO3a-dependent apoptosis in prostate cancer. Cell Death Dis 7, e2111 (2016).

313. Rayego-Mateos, S. et al. TWEAK transactivation of the epidermal growth factor receptor mediates renal inflammation. Journal of Pathology 231, 480-494 (2013).

314. Papadakis, E.S. et al. Axl Promotes Cutaneous Squamous Cell Carcinoma Survival through Negative Regulation of Pro-Apoptotic Bcl-2 Family Members. J Invest Dermatol 131, 509-517 (2011).

315. Zhu, H.Y. et al. Peroxisome proliferator-activated receptor-gamma agonist troglitazone suppresses transforming growth factor-beta 1 signalling through miR-92b upregulation-inhibited Axl expression in human keloid fibroblasts in vitro. Am J Transl Res 8, 3460-3470 (2016). 
316. Carroll, D. A CRISPR Approach to Gene Targeting. Mol Ther 20, 1658-1660 (2012).

317. Sander, J.D. \& Joung, J.K. CRISPR-Cas systems for editing, regulating and targeting genomes. Nat Biotechnol 32, 347-355 (2014).

318. Xue, H.Y. et al. In vivo gene therapy potentials of CRISPR-Cas9. Gene Ther 23, 557-559 (2016).

319. Long, C.Z. et al. Prevention of muscular dystrophy in mice by CRISPR/Cas9-mediated editing of germline DNA. Science 345, 1184-1188 (2014). 


\section{Vita}

Dhananjay Suresh was born in Bhilai, India on December 22, 1986. He finished his schooling in many states of India. He finished his high school from Kota, Rajasthan, India. He went to receive his B.S in Biotechnology from the SRM University in 2008. He earned his Ph.D. degree in Bioengineering from the University of Missouri in December 2016. 